\title{
THE NOTION OF $N=1$ SUPERGEOMETRIC VERTEX OPERATOR SUPERALGEBRA AND THE ISOMORPHISM THEOREM
}

\author{
KATRINA BARRON
}

\begin{abstract}
We introduce the notion of $N=1$ supergeometric vertex operator superalgebra motivated by the geometry underlying genus-zero, twodimensional, holomorphic $N=1$ superconformal field theory. We then show, assuming the convergence of certain projective factors, that the category of such objects is isomorphic to the category of $N=1$ Neveu-Schwarz vertex operator superalgebras.
\end{abstract}

\section{Introduction and Preliminaries}

1.1. Introduction. In this paper, we introduce the notion of $N=1$ supergeometric vertex operator superalgebra and prove that the category of such objects is isomorphic to the category of $N=1$ Neveu-Schwarz vertex operator superalgebras with (or without) odd formal variables [B3]. The notion of $N=1$ supergeometric vertex operator superalgebra is based on the geometric structure of the moduli space of $N=1$ superspheres with tubes along with a sewing operation on this moduli space and an action of the symmetric groups [B4]. This moduli space is the fundamental geometric structure underlying genus-zero, two-dimensional, holomorphic $N=1$ superconformal field theory.

Conformal field theory (or more specifically, string theory) and related theories such as superconformal field theories (cf. [BPZ, [FS], [V], and [S] ) are the most promising attempts at developing a physical theory that combines all fundamental interactions of particles, including gravity. The geometry of this theory extends the use of Feynman diagrams, describing the interactions of point particles whose propagation in time sweeps out a line in space-time, to one-dimensional "particles" (strings) whose propagation in time sweeps out a two-dimensional surface. For two-dimensional genus-zero holomorphic conformal field theory, algebraically, these interactions can be described by products of vertex operators or more precisely, by vertex operator algebras (cf. [FLM]).

Independently from the physical theory of conformal fields, vertex operator algebras arose naturally from the study of representations of infinite-dimensional Lie algebras and in the construction of representations of the Monster finite simple group FLM. However, it wasn't until 1990 that a rigorous mathematical interpretation of the geometry of particle interactions and the algebraic description of

1991 Mathematics Subject Classification. Primary 17B68, 17B69, 17B81, 81R10, 81T40.

Key words and phrases. Vertex operator superalgebras, superconformal field theory.

The author was supported in part by an AAUW Educational Foundation American Dissertation Fellowship and an NSF Mathematical Sciences Postdoctoral Research Fellowship. 
those interactions via vertex operator algebras was realized. In [H1] and [H2], motivated by the geometric notions arising in conformal field theory, Huang gives a precise geometric interpretation of the notion of vertex operator algebra thus giving a rigorous basis for the geometric and algebraic "sewing" together of particle interactions.

In $\mathrm{Fd}$, Friedan describes the extension of the physical model of conformal field theory to that of superconformal field theory with one fermionic component $(N=1)$, and the notion of a superstring whose propagation in time sweeps out a supersurface is introduced. Whereas conformal field theory attempts to describe the interactions of bosons, $N=1$ superconformal field theory attempts to describe the interactions of boson-fermion pairs and has been discovered to be essential for a full description of all particle interactions. This theory requires an operator $D$ such that $D^{2}=\partial / \partial z$. Such an operator arises naturally in supergeometry.

Within the framework of supergeometry (cf. $[\mathrm{D},[\mathrm{R}]$ and $[\mathrm{CR}]$ ) and motivated by $N=1$ superconformal field theory, in this paper, we extend the work of Huang to an $N=1$ supergeometric interpretation of $N=1$ Neveu-Schwarz vertex operator superalgebras. This paper relies heavily on the results of [B4], and the notion and notation of $N=1$ Neveu-Schwarz vertex operator superalgebra $(N=1$ NS-VOSA $)$ as presented in B3. In [B4], we studied the moduli space of $N=1$ superspheres with tubes and defined a sewing operation on this moduli space. This sewing operation defines a partial-operad structure on the moduli space (cf. [M], HL1, HL2 ), and in B4, we thoroughly analyze this structure both algebraically and analytically. The most important aspect of this work is that given two canonical $N=1$ superspheres with tubes representing equivalence classes in the moduli space, we can completely determine the resulting sewn $N=1$ supersphere as being in the equivalence class of a specific canonical $N=1$ supersphere. This is done by explicitly solving the sewing equation and thus constructing the uniformizing function which takes the resulting sewn $N=1$ supersphere to the canonical $N=1$ supersphere representative.

In this paper, assuming the convergence of a certain series, denoted $\Gamma$, (see Conjecture 3.5], we define an algebra over this partial operad of the moduli space of $N=1$ superspheres with tubes and the sewing operation and extended to include the projective factors associated to $\Gamma$, and call such an algebra an $N=1$ supergeometric vertex operator superalgebra $(N=1 S G$-VOSA). We then prove that the category of such objects is isomorphic to the category of (algebraic) $N=1$ NS-VOSAs. The fact that $N=1$ SG-VOSAs are defined over the geometric structure of the moduli space with sewing, and that the sewing operation is completely soluble, provides much more information for the corresponding algebraic structure of an $N=1$ SG-VOSA, and thus by isomorphism of categories, for the more familiar structure of an $N=1$ NS-VOSA as well. This includes a full understanding of the effects of a change of coordinates for $N=1$ NS-VOSA as studied in B5], as well as the development of alternate notions of superconformality and relations to $N=2$ superconformal field theory as studied in [B6]. In addition, this fully realized genus-zero case is the first step toward understanding higher genus $N=1$ superconformal field theory.

This paper is organized as follows. In the remainder of this section, Section 1, we give a brief summary of some basic definitions. In Sections 2 and 3, we give an overview of the results of [B4]. This overview is only meant to recall these results 
for the reader who is already familiar with [B4]. Certainly for a full understanding of these results, it is essential that one refer to B4.

In Section 4, we discuss (1/2)Z-graded vector spaces with finite-dimensional homogeneous subspaces and define the $t^{1 / 2}$-contraction of $n$-ary functions from such a vector space to its algebraic completion. This contraction of functions can be thought of as being analogous to the sewing operation on the moduli space of superspheres with tubes in that both contraction and sewing define the formal substitution maps in the definition of a partial (or partial pseudo-) operad (cf. HL1, HL2, H2 ). In the second subsection of Section 4, we introduce the notion of $N=1$ supergeometric vertex operator superalgebra ( $N=1 S G$-VOSA) assuming the convergence of the projective factors for the central charge.

In Section 5, we recall from B3] the notion of $N=1$ Neveu-Schwarz vertex operator superalgebra ( $N=1 \mathrm{NS}$-VOSA) and several consequences of the definition including the properties of associativity and supercommutativity.

In Section [ we construct an $N=1$ NS-VOSA with odd formal variables from an $N=1$ SG-VOSA, and in Section 7 we construct an $N=1$ SG-VOSA from an $N=1$ NS-VOSA with odd formal variables.

In Section 8 we prove that the category of $N=1 \mathrm{SG}-\mathrm{VOSAs}$ is isomorphic to the category of $N=1$ NS-VOSAs with odd formal variables.

The results of this paper were first announced in B1] as part of the authors Ph.D. thesis B2].

1.2. Grassmann algebras and the $N=1$ Neveu-Schwarz algebra. Let $\mathbb{Z}_{2}$ denote the integers modulo two. For a $\mathbb{Z}_{2}$-graded vector space $V=V^{0} \oplus V^{1}$, define the sign function $\eta$ on the homogeneous subspaces of $V$ by $\eta(v)=i$ for $v \in V^{i}$, $i=0,1$. If $\eta(v)=0$, we say that $v$ is even, and if $\eta(v)=1$, we say that $v$ is odd.

A superalgebra is an (associative) algebra $A$ (with identity $1 \in A$ ), such that: (i) $A$ is a $\mathbb{Z}_{2}$-graded algebra; (ii) $a b=(-1)^{\eta(a) \eta(b)} b a$ for $a, b$ homogeneous in $A$.

The exterior algebra over a vector space $U$, denoted $\Lambda(U)$, has the structure of a superalgebra. Fix $U_{L}$ to be an $L$-dimensional vector space over $\mathbb{C}$ for $L \in \mathbb{N}$ with fixed basis $\left\{\zeta_{1}, \ldots, \zeta_{L}\right\}$ such that $U_{L} \subset U_{L+1}$. We denote $\Lambda\left(U_{L}\right)$ by $\bigwedge_{L}$ and call this the Grassmann algebra on $L$ generators. Note that $\Lambda_{L} \subset \bigwedge_{L+1}$, and taking the direct limit as $L \rightarrow \infty$, we have the infinite Grassmann algebra denoted by $\Lambda_{\infty}$. We use the notation $\bigwedge_{*}$ to denote a Grassmann algebra, finite or infinite.

The $\mathbb{Z}_{2}$-grading of $\bigwedge_{*}$ is given explicitly by

$$
\begin{aligned}
& \bigwedge_{*}^{0}=\left\{a \in \bigwedge_{*} \mid a=\sum_{(i) \in I_{*}} a_{(i)} \zeta_{i_{1}} \zeta_{i_{2}} \cdots \zeta_{i_{2 n}}, a_{(i)} \in \mathbb{C}, n \in \mathbb{N}\right\} \\
& \bigwedge_{*}^{1}=\left\{a \in \bigwedge_{*} \mid a=\sum_{(j) \in J_{*}} a_{(j)} \zeta_{j_{1}} \zeta_{j_{2}} \cdots \zeta_{j_{2 n+1}}, a_{(j)} \in \mathbb{C}, n \in \mathbb{N}\right\},
\end{aligned}
$$

where

$$
\begin{aligned}
& I_{*}=\left\{(i)=\left(i_{1}, i_{2}, \ldots, i_{2 n}\right) \mid i_{1}<i_{2}<\cdots<i_{2 n}, i_{l} \in\{1,2, \ldots, *\}, n \in \mathbb{N}\right\}, \\
& J_{*}=\left\{(j)=\left(j_{1}, j_{2}, \ldots, j_{2 n+1}\right) \mid j_{1}<j_{2}<\cdots<j_{2 n+1}, j_{l} \in\{1,2, \ldots, *\}, n \in \mathbb{N}\right\},
\end{aligned}
$$

with $\{1,2, \ldots, *\}$ denoting $\{1,2, \ldots, L\}$ if $\bigwedge_{*}$ is the finite-dimensional Grassmann algebra $\Lambda_{L}$ and denoting the positive integers $\mathbb{Z}_{+}$if $\Lambda_{*}=\Lambda_{\infty}$. We can also 
decompose $\bigwedge_{*}$ into body, $\left(\bigwedge_{*}\right)_{B}=\left\{a_{(\emptyset)} \in \mathbb{C}\right\}$, and soul

$$
\left(\bigwedge_{*}\right)_{S}=\left\{a \in \bigwedge_{*} \mid a=\sum_{\substack{(k) \in I_{*} \cup J_{*} \\ k \neq(\emptyset)}} a_{(k)} \zeta_{k_{1}} \zeta_{k_{2}} \cdots \zeta_{k_{n}}, a_{(k)} \in \mathbb{C}\right\}
$$

subspaces such that $\bigwedge_{*}=\left(\bigwedge_{*}\right)_{B} \oplus\left(\bigwedge_{*}\right)_{S}$. For $a \in \bigwedge_{*}$, we write $a=a_{B}+a_{S}$ for its body and soul decomposition.

A $\mathbb{Z}_{2}$-graded vector space $\mathfrak{g}$ is said to be a Lie superalgebra if it has a bilinear operation $[\cdot, \cdot]$ such that for $u, v$ homogeneous in $\mathfrak{g}$ : (i) $[u, v] \in \mathfrak{g}^{(\eta(u)+\eta(v)) \bmod 2 \text {; }}$ (ii) skew-symmetry holds $[u, v]=-(-1)^{\eta(u) \eta(v)}[v, u]$; (iii) the Jacobi identity holds $(-1)^{\eta(u) \eta(w)}[[u, v], w]+(-1)^{\eta(v) \eta(u)}[[v, w], u]+(-1)^{\eta(w) \eta(v)}[[w, u], v]=0$.

Given a Lie superalgebra $\mathfrak{g}$ and a superalgebra $A$, the space $\left(A^{0} \otimes \mathfrak{g}^{0}\right) \oplus\left(A^{1} \otimes \mathfrak{g}^{1}\right)$ is a Lie algebra with bracket given by $[a u, b v]=(-1)^{\eta(b) \eta(u)} a b[u, v]$ for $a, b \in A$ and $u, v \in \mathfrak{g}$ homogeneous. We call $\left(A^{0} \otimes \mathfrak{g}^{0}\right) \oplus\left(A^{1} \otimes \mathfrak{g}^{1}\right)$ the $A$-envelope of $\mathfrak{g}$. Similarly, given two superalgebras $A$ and $\hat{A}$, we can form the $A$-envelope of $\hat{A}$ given by $\left(A^{0} \otimes \hat{A}^{0}\right) \oplus\left(A^{1} \otimes \hat{A}^{1}\right)$ which is naturally an algebra.

For any $\mathbb{Z}_{2}$-graded associative algebra $A$ and for $u, v \in A$ of homogeneous sign, we can define $[u, v]=u v-(-1)^{\eta(u) \eta(v)} v u$, making $A$ into a Lie superalgebra. The algebra of endomorphisms of $A$, denoted End $A$, has a natural $\mathbb{Z}_{2}$-grading induced from that of $A$, and defining $[X, Y]=X Y-(-1)^{\eta(X) \eta(Y)} Y X$ for $X, Y$ homogeneous in End $A$, this gives End $A$ a Lie superalgebra structure. An element $D \in(\text { End } A)^{i}$, for $i \in \mathbb{Z}_{2}$, is called a superderivation of sign $i$ (denoted $\eta(D)=i$ ) if $D$ satisfies the super-Leibniz rule $D(u v)=(D u) v+(-1)^{\eta(D) \eta(u)} u D v$ for $u, v \in A$ homogeneous.

The Virasoro algebra is the Lie algebra with basis consisting of the central element $d$ and $L_{n}$, for $n \in \mathbb{Z}$, and commutation relations

$$
\left[L_{m}, L_{n}\right]=(m-n) L_{m+n}+\frac{1}{12}\left(m^{3}-m\right) \delta_{m+n, 0} d,
$$

for $m, n \in \mathbb{Z}$. The $N=1$ Neveu-Schwarz Lie superalgebra, denoted $\mathfrak{n} \mathfrak{s}$, is a superextension of the Virasoro algebra by the odd elements $G_{n+1 / 2}$, for $n \in \mathbb{Z}$, with supercommutation relations

$$
\begin{aligned}
{\left[G_{m+\frac{1}{2}}, L_{n}\right] } & =\left(m-\frac{n-1}{2}\right) G_{m+n+\frac{1}{2}} \\
{\left[G_{m+\frac{1}{2}}, G_{n-\frac{1}{2}}\right] } & =2 L_{m+n}+\frac{1}{3}\left(m^{2}+m\right) \delta_{m+n, 0} d
\end{aligned}
$$

in addition to (1.1).

1.3. Superanalytic and superconformal superfunctions. Let $U$ be a subset of $\bigwedge_{*}$, and write $U=U^{0} \oplus U^{1}$ for the decomposition of $U$ into even and odd subspaces. Let $z$ be an even variable in $U^{0}$ and $\theta$ an odd variable in $U^{1}$. We call $H: U \longrightarrow \bigwedge_{*}$, mapping $(z, \theta) \mapsto H(z, \theta)$, a $\bigwedge_{*}$-superfunction on $U$ in (1,1)-variables.

Let $z_{B}$ be a complex variable and $h\left(z_{B}\right)$ a complex analytic function in some open set $U_{B} \subset \mathbb{C}$. For $z$ a variable in $\bigwedge_{*}^{0}$, we define $h(z)$ to be the Taylor expansion about the body of $z=z_{B}+z_{S}$. Then $h(z)$ is well defined (i.e., convergent) in the open neighborhood $\left\{z=z_{B}+z_{S} \in \bigwedge_{*}^{0} \mid z_{B} \in U_{B}\right\}=U_{B} \times\left(\bigwedge_{*}^{0}\right)_{S} \subseteq \bigwedge_{*}^{0}$. Since $h(z)$ is algebraic in each $z_{(i)}$, for $(i) \in I_{*}$, it follows that $h(z)$ is complex analytic in each of the complex variables $z_{(i)}$.

For $n \in \mathbb{N}$, we introduce the notation $\bigwedge_{*>n}$ to denote a finite Grassmann algebra $\bigwedge_{L}$ with $L>n$ or an infinite Grassmann algebra. We will use the corresponding 
index notations for the corresponding indexing sets $I_{*>n}$ and $J_{*>n}$. A superanalytic $\bigwedge_{*>0}$-superfunction in $(1,1)$-variables $H$ is a $\bigwedge_{*>0^{-}}$-superfunction in $(1,1)$-variables of the form

$$
\begin{aligned}
H(z, \theta)= & (f(z)+\theta \xi(z), \psi(z)+\theta g(z)) \\
= & \left(\sum_{(i) \in I_{*-1}} f_{(i)}(z) \zeta_{i_{1}} \zeta_{i_{2}} \cdots \zeta_{i_{n}}+\theta \sum_{(j) \in J_{*-1}} \xi_{(j)}(z) \zeta_{j_{1}} \zeta_{j_{2}} \cdots \zeta_{j_{2 n+1}},\right. \\
& \left.\sum_{(j) \in J_{*-1}} \psi_{(j)}(z) \zeta_{j_{1}} \zeta_{j_{2}} \cdots \zeta_{j_{2 n+1}}+\theta \sum_{(i) \in I_{*-1}} g_{(i)}(z) \zeta_{i_{1}} \zeta_{i_{2}} \cdots \zeta_{i_{n}}\right)
\end{aligned}
$$

where $f_{(i)}\left(z_{B}\right), g_{(i)}\left(z_{B}\right), \xi_{(j)}\left(z_{B}\right)$, and $\psi_{(j)}\left(z_{B}\right)$ are all complex analytic in some non-empty open subset $U_{B} \subseteq \mathbb{C}$. If each $f_{(i)}\left(z_{B}\right), g_{(i)}\left(z_{B}\right), \xi_{(j)}\left(z_{B}\right)$, and $\psi_{(j)}\left(z_{B}\right)$ is complex analytic in $U_{B} \subseteq \mathbb{C}$, then $H(z, \theta)$ is well defined (i.e., convergent) for $\left\{(z, \theta) \in \bigwedge_{*>0} \mid z_{B} \in U_{B}\right\}=U_{B} \times\left(\bigwedge_{*>0}\right)_{S}$. Consider the topology on $\bigwedge_{*}$ given by the product of the usual topology on $\left(\bigwedge_{*}\right)_{B}=\mathbb{C}$ and the trivial topology on $\left(\bigwedge_{*}\right)_{S}$. This topology on $\bigwedge_{*}$ is called the DeWitt topology. The natural domain of any superanalytic $\bigwedge_{*>0}$-superfunction is an open set in the DeWitt topology on $\bigwedge_{*>0}$.

Since $1 / a=\sum_{n \in \mathbb{N}}(-1)^{n} a_{S}^{n} / a_{B}^{n+1}$ is well defined if and only if $a_{B} \neq 0$, the set of invertible elements in $\bigwedge_{*}$, denoted $\bigwedge_{*}^{\times}$, is given by $\bigwedge_{*}^{\times}=\left\{a \in \Lambda_{*} \mid a_{B} \neq 0\right\}$. Note that $\bigwedge_{*}^{\times} \subset \Lambda_{*}$ is open in the DeWitt topology.

We define the (left) partial derivatives $\partial / \partial z$ and $\partial / \partial \theta$ acting on superfunctions which are superanalytic in some DeWitt open neighborhood $U$ of $(z, \theta) \in \bigwedge_{*>0}$ by

$$
\begin{aligned}
\Delta z\left(\frac{\partial}{\partial z} H(z, \theta)\right)+O\left((\Delta z)^{2}\right) & =H(z+\Delta z, \theta)-H(z, \theta) \\
\Delta \theta\left(\frac{\partial}{\partial \theta} H(z, \theta)\right) & =H(z, \theta+\Delta \theta)-H(z, \theta)
\end{aligned}
$$

for all $\Delta z \in \bigwedge_{*>0}^{0}$ and $\Delta \theta \in \bigwedge_{*>0}^{1}$ such that $z+\Delta z \in U^{0}=U_{B} \times\left(\bigwedge_{*>0}^{0}\right)_{S}$ and $\theta+\Delta \theta \in U^{1}=\bigwedge_{*>0}^{1}$. Note that $\partial / \partial z$ and $\partial / \partial \theta$ are endomorphisms of the superalgebra of superanalytic $(1,1)$-superfunctions, and in fact, are even and odd superderivations, respectively.

If $h\left(z_{B}\right)$ is complex analytic in an open neighborhood of the complex plane, then $h\left(z_{B}\right)$ has a Laurent series expansion in $z_{B}$, given by $h\left(z_{B}\right)=\sum_{l \in \mathbb{Z}} c_{l} z_{B}^{l}$, for $c_{l} \in \mathbb{C}$, and we have $h(z)=\sum_{n \in \mathbb{N}}\left(z_{S}^{n} / n !\right) h^{(n)}\left(z_{B}\right)=\sum_{l \in \mathbb{Z}} c_{l}\left(z_{B}+z_{S}\right)^{l}=\sum_{l \in \mathbb{Z}} c_{l} z^{l}$ where $\left(z_{B}+z_{S}\right)^{l}$, for $l \in \mathbb{Z}$, is always understood to mean expansion in positive powers of the second variable, in this case $z_{S}$. Thus if $H$ is a $\bigwedge_{*>0}$-superfunction in $(1,1)$-variables which is superanalytic in a (DeWitt) open neighborhood, $H$ can be expanded as

$$
H(z, \theta)=\left(\sum_{l \in \mathbb{Z}} a_{l} z^{l}+\theta \sum_{l \in \mathbb{Z}} n_{l} z^{l}, \sum_{l \in \mathbb{Z}} m_{l} z^{l}+\theta \sum_{l \in \mathbb{Z}} b_{l} z^{l}\right)
$$

for $a_{l}, b_{l} \in \bigwedge_{*-1}^{0}$ and $m_{l}, n_{l} \in \bigwedge_{*-1}^{1}$.

Define $D$ to be the odd superderivation $D=\partial / \partial \theta+\theta \partial / \partial z$ acting on superanalytic $\bigwedge_{*>0}$-superfunctions in $(1,1)$-variables. Then $D^{2}=\partial / \partial z$, and if $H(z, \theta)=(\tilde{z}, \tilde{\theta})$ is superanalytic in some DeWitt open subset, then $D$ transforms 
under $H(z, \theta)$ by $D=(D \tilde{\theta}) \tilde{D}+(D \tilde{z}-\tilde{\theta} D \tilde{\theta}) \tilde{D}^{2}$. We define a superconformal $(1,1)$ superfunction on a DeWitt open subset $U$ of $\bigwedge_{*>0}$ to be a superanalytic superfunction $H$ under which $D$ transforms homogeneously of degree one. Thus a superanalytic function $H(z, \theta)=(\tilde{z}, \tilde{\theta})$ is superconformal if and only if, in addition to being superanalytic, $H$ satisfies $D \tilde{z}-\tilde{\theta} D \tilde{\theta}=0$, for $D \tilde{\theta}$ not identically zero, thus transforming $D$ by $D=(D \tilde{\theta}) \tilde{D}$.

1.4. Super-Riemann surfaces. A DeWitt $(1,1)$-dimensional manifold over $\bigwedge_{*}$ is a topological space $X$ with a countable basis which is locally homeomorphic to an open subset of $\bigwedge_{*}$ in the DeWitt topology. A DeWitt $(1,1)$-chart on $X$ over $\bigwedge_{*}$ is a pair $(U, \Omega)$ such that $U$ is an open subset of $X$ and $\Omega$ is a homeomorphism of $U$ onto an open subset of $\Lambda_{*}^{0}$ in the DeWitt topology. A superanalytic atlas of DeWitt $(1,1)$-charts on $X$ over $\bigwedge_{*>0}$ is a family of charts $\left\{\left(U_{\alpha}, \Omega_{\alpha}\right)\right\}_{\alpha \in A}$ satisfying: (i) each $U_{\alpha}$ is open in $X$, and $\bigcup_{\alpha \in A} U_{\alpha}=X$; (ii) each $\Omega_{\alpha}$ is a homeomorphism from $U_{\alpha}$ to a (DeWitt) open set in $\bigwedge_{*>0}$ such that $\Omega_{\alpha} \circ \Omega_{\beta}^{-1}: \Omega_{\beta}\left(U_{\alpha} \cap U_{\beta}\right) \longrightarrow$ $\Omega_{\alpha}\left(U_{\alpha} \cap U_{\beta}\right)$ is superanalytic for all non-empty $U_{\alpha} \cap U_{\beta}$. Such an atlas is called maximal if, given any chart $(U, \Omega)$ such that $\Omega \circ \Omega_{\beta}^{-1}: \Omega_{\beta}\left(U \cap U_{\beta}\right) \longrightarrow \Omega\left(U \cap U_{\beta}\right)$ is a superanalytic homeomorphism for all $\beta$, then $(U, \Omega) \in\left\{\left(U_{\alpha}, \Omega_{\alpha}\right)\right\}_{\alpha \in A}$. A DeWitt $(1,1)$-supermanifold over $\Lambda_{*>0}$ is a $\operatorname{DeWitt}(1,1)$-dimensional topological space $M$ together with a maximal superanalytic atlas of DeWitt $(1,1)$-charts over $\bigwedge_{*>0}$.

Given a DeWitt $(1,1)$-supermanifold $M$ over $\bigwedge_{*>0}$, define an equivalence relation $\sim$ on M by letting $p \sim q$ if and only if there exists $\alpha \in A$ such that $p, q \in U_{\alpha}$ and $\pi_{B}\left(\Omega_{\alpha}(p)\right)=\pi_{B}\left(\Omega_{\alpha}(q)\right)$ where $\pi_{B}$ is the canonical projection of $\bigwedge_{*>0}$ onto its body. Let $p_{B}$ denote the equivalence class of $p$ under this equivalence relation. Define the body $M_{B}$ of $M$ to be the one-dimensional complex manifold with analytic structure given by the coordinate charts $\left\{\left(\left(U_{\alpha}\right)_{B},\left(\Omega_{\alpha}\right)_{B}\right)\right\}_{\alpha \in A}$ where $\left(U_{\alpha}\right)_{B}=\left\{p_{B} \mid p \in U_{\alpha}\right\}$, and $\left(\Omega_{\alpha}\right)_{B}:\left(U_{\alpha}\right)_{B} \longrightarrow \mathbb{C}$ is given by $\left(\Omega_{\alpha}\right)_{B}\left(p_{B}\right)=\pi_{B} \circ \Omega_{\alpha}(p)$.

For any DeWitt $(1,1)$-supermanifold $M$, its body $M_{B}$ is a Riemann surface. A super-Riemann surface over $\bigwedge_{*>0}$ is a DeWitt $(1,1)$-supermanifold over $\bigwedge_{*>0}$ with coordinate atlas $\left\{\left(U_{\alpha}, \Omega_{\alpha}\right)\right\}_{\alpha \in A}$ such that the coordinate transition functions $\Omega_{\alpha} \circ \Omega_{\beta}^{-1}$ in addition to being superanalytic are also superconformal for all nonempty $U_{\alpha} \cap U_{\beta}$. Since the condition that the coordinate transition functions be superconformal instead of merely superanalytic is such a strong condition (unlike in the nonsuper case), we again stress the distinction between a supermanifold which has superanalytic transition functions versus a super-Riemann surface which has superconformal transition functions.

\section{The MOduli SPACE OF $N=1$ SUPerspheres With tUbes AND The SEWING OPERATION}

In [B4, we define the moduli space $S K(n)$ of $N=1$ superspheres with $n$ incoming tubes and one outgoing tube, define a sewing operation on this space and give a detailed algebraic and analytic study of this space with this sewing operation. In this section, we recall some results of this work that we will need in order to define the notion of $N=1$ supergeometric vertex operator superalgebra and to prove the Isomorphism Theorem.

2.1. The moduli space of $N=1$ superspheres with tubes. By $N=1 \mathrm{su}$ persphere we will mean a (superconformal) super-Riemann surface over $\bigwedge_{*>0}$ such 
that its body is a genus-zero one-dimensional connected compact complex manifold. From now on we will refer to such an object as a supersphere.

A supersphere with $1+n$ tubes for $n \in \mathbb{N}$, is a supersphere $S$ with 1 negatively oriented point $p_{0}$ and $n$ positively oriented points $p_{1}, \ldots, p_{n}$ (we call them punctures) on $S$ which all have distinct bodies and with local superconformal coordinates $\left(U_{0}, \Omega_{0}\right), \ldots,\left(U_{n}, \Omega_{n}\right)$ vanishing at the punctures $p_{0}, \ldots, p_{n}$, respectively. We denote this structure by $\left(S ; p_{0}, \ldots, p_{n} ;\left(U_{0}, \Omega_{0}\right), \ldots,\left(U_{n}, \Omega_{n}\right)\right)$. We will always order the punctures so that the negatively oriented puncture is $p_{0}$.

Let $S_{1}=\left(S_{1} ; p_{0}, \ldots, p_{m} ;\left(U_{0}, \Omega_{0}\right), \ldots,\left(U_{m}, \Omega_{m}\right)\right)$ be a supersphere with $1+m$ tubes, for $m \in \mathbb{N}$, and let $S_{2}=\left(S_{2} ; q_{0}, \ldots, q_{n} ;\left(V_{0}, \Xi_{0}\right), \ldots,\left(V_{n}, \Xi_{n}\right)\right)$ be a supersphere with $1+n$ tubes, for $n \in \mathbb{N}$. A map $F: S_{1} \rightarrow S_{2}$ will be said to be superconformal if $\Xi_{\beta} \circ F \circ \Omega_{\alpha}^{-1}$ is superconformal for all charts $\left(U_{\alpha}, \Omega_{\alpha}\right)$ of $S_{1}$, for all charts $\left(V_{\beta}, \Xi_{\beta}\right)$ of $S_{2}$, and for all $(z, \theta) \in \Omega_{\alpha}\left(U_{\alpha}\right)$ such that $F \circ \Omega_{\alpha}^{-1}(z, \theta) \in V_{\beta}$. If $m=n$ and there is a superconformal isomorphism $F: S_{1} \rightarrow S_{2}$ such that for each $j=0, \ldots, n$, we have that $F\left(p_{j}\right)=q_{j}$ and $\left.\Omega_{j}\right|_{W_{j}}=\left.\Xi_{j} \circ F\right|_{W_{j}}$, for $W_{j}$ some DeWitt neighborhood of $p_{j}$, then we say that these two superspheres with $1+n$ tubes are superconformally equivalent and $F$ is a superconformal equivalence from $S_{1}$ to $S_{2}$. Thus the superconformal equivalence class of a supersphere with tubes depends only on the supersphere, the punctures, and the germs of the local coordinate maps vanishing at the punctures.

The collection of all superconformal equivalence classes of superspheres over $\bigwedge_{*>0}$ with $1+n$ tubes, for $n \in \mathbb{N}$, is called the moduli space of superspheres over $\bigwedge_{*>0}$ with $1+n$ tubes. The collection of all superconformal equivalence classes of superspheres over $\bigwedge_{*>0}$ with tubes is called the moduli space of superspheres over $\bigwedge_{*>0}$ with tubes.

Let $S \hat{\mathbb{C}}$ be the supersphere with superconformal structure given by the covering of local coordinate neighborhoods $\left\{U_{\Delta}, U_{\Upsilon}\right\}$ and the local coordinate maps $\Delta$ : $U_{\Delta} \longrightarrow \bigwedge_{*>0}$ and $\Upsilon: U_{\Upsilon} \longrightarrow \bigwedge_{*>0}$, which are homeomorphisms of $U_{\Delta}$ onto $\bigwedge_{*>0}$ and $U_{\Upsilon}$ onto $\bigwedge_{*>0}$, respectively, such that $\Delta \circ \Upsilon^{-1}: \bigwedge_{*>0}^{\times} \longrightarrow \bigwedge_{*>0}^{\times}$is given by $(z, \theta) \mapsto(1 / z, i \theta / z)=\Delta \circ \Upsilon^{-1}(z, \theta)=I(z, \theta)$. Thus the body of $S \hat{\mathbb{C}}$ is the Riemann sphere, $(S \hat{\mathbb{C}})_{B}=\hat{\mathbb{C}}=\mathbb{C} \cup\{\infty\}$, with coordinates $z_{B}$ near 0 and $1 / z_{B}$ near $\infty$. We will call $S \hat{\mathbb{C}}$ the super-Riemann sphere and will refer to $\Upsilon^{-1}(0)$ as the point at $(\infty, 0)$ or just the point at infinity and to $\Delta^{-1}(0)$ as the point at $(0,0)$ or just the point at zero.

Let $T: S \hat{\mathbb{C}} \longrightarrow S \hat{\mathbb{C}}$ be a superconformal automorphism. Then defining $T_{\Delta}=$ $\Delta \circ T \circ \Delta^{-1}$, we have that $T$ must be a superprojective transformation satisfying

$$
\begin{aligned}
T_{\Delta}: \bigwedge_{*>0} \backslash\left(\left\{(-d / c)_{B}\right\}\right. & \left.\times\left(\bigwedge_{*>0}\right)_{S}\right) \longrightarrow \bigwedge_{*>0} \backslash\left(\left\{(a / c)_{B}\right\} \times\left(\bigwedge_{*>0}\right)_{S}\right) \\
(z, \theta) & \mapsto\left(\frac{a z+b}{c z+d}+\theta \frac{\gamma z+\delta}{(c z+d)^{2}}, \frac{\gamma z+\delta}{c z+d}+\theta \frac{1+\frac{1}{2} \delta \gamma}{c z+d}\right),
\end{aligned}
$$

for $a, b, c, d \in \bigwedge_{*-1}^{0}, \gamma, \delta \in \bigwedge_{*-1}^{1}$ and $a d-b c=1$, and $T$ is determined uniquely by $T_{\Delta}$.

In $\mathrm{CR}$, Crane and Rabin prove the uniformization theorem for super-Riemann surfaces. For super-Riemann surfaces with genus-zero compact bodies, the theorem states that any such super-Riemann surface is superconformally equivalent to the super-Riemann sphere $S \hat{\mathbb{C}}$. Thus as is proved in [B4], any supersphere with $1+n$ 
tubes is superconformally equivalent to

$$
\left(S \hat{\mathbb{C}} ; \infty,\left(z_{1}, \theta_{1}\right), \ldots,\left(z_{n-1}, \theta_{n-1}\right), 0 ; H_{0}, H_{1}, \ldots, H_{n}\right),
$$

where $\left(z_{0}, \theta_{0}\right)=\infty=\Upsilon^{-1}(0)$ is the coordinate of the outgoing puncture, $\left(z_{n}, \theta_{n}\right)=$ $0=\Delta^{-1}(0)$ is the coordinate of the last incoming puncture, $\left(z_{i}, \theta_{i}\right) \in\left(\bigwedge_{*>0}\right)^{\times}$with $\left(z_{i}\right)_{B} \neq\left(z_{j}\right)_{B}$ for $i \neq j$, and $H_{i}$ is a coordinate from an open set around the $i$-th puncture to $\bigwedge_{*>0}$ which maps the puncture to zero.

We now temporarily switch to a formal algebraic setting in order to better understand superconformal local coordinates. Let $R$ be a superalgebra, let $x$ be an even formal variable, and let $\varphi$ be an odd formal variable. By this we mean that $x$ commutes with all formal variables and all superalgebra elements and $\varphi$ anticommutes with all odd formal variables and all odd superalgebra elements but commutes with even elements. Note that $R((x))[\varphi]$ and $R\left(\left(x^{-1}\right)\right)[\varphi]$ are superalgebras. For $j \in \mathbb{Z}$, consider the even superderivations

$$
L_{j}(x, \varphi)=-\left(x^{j+1} \frac{\partial}{\partial x}+\left(\frac{j+1}{2}\right) \varphi x^{j} \frac{\partial}{\partial \varphi}\right)
$$

and the odd superderivations

$$
G_{j-\frac{1}{2}}(x, \varphi)=-x^{j}\left(\frac{\partial}{\partial \varphi}-\varphi \frac{\partial}{\partial x}\right)
$$

in $\left.\left.\operatorname{Der}\left(R\left[\left[x, x^{-1}\right]\right]\right)\right)[\varphi]\right)$. Then $L_{j}(x, \varphi)$ and $G_{j-1 / 2}(x, \varphi)$ give a representation of the $N=1$ Neveu-Schwarz Lie superalgebra, $\mathfrak{n} \mathfrak{s}$, with central charge zero.

For any formal power series $H \in R\left[\left[x, x^{-1}\right]\right][\varphi]$, we say that $H(x, \varphi)=(\tilde{x}, \tilde{\varphi})$ is superconformal if $D \tilde{x}=\tilde{\varphi} D \tilde{\varphi}$ for $D=\partial / \partial \varphi+\varphi \partial / \partial x$.

Let $\left(R^{0}\right)^{\times}$denote the invertible elements in $R^{0}$. Let $\left(R^{0}\right)^{\infty}$ be the set of all sequences $A=\left\{A_{j}\right\}_{j \in \mathbb{Z}_{+}}$of even elements in $R$, let $\left(R^{1}\right)^{\infty}$ be the set of all sequences $M=\left\{M_{j-1 / 2}\right\}_{j \in \mathbb{Z}_{+}}$of odd elements in $R$, and let $R^{\infty}=\left(R^{0}\right)^{\infty} \oplus\left(R^{1}\right)^{\infty}$. In B2] and [B4], we show that there is a bijection between the set of formal superconformal power series $H \in R\left[\left[x, x^{-1}\right]\right][\varphi]$ which vanish at zero, i.e., formal superconformal power series of the form

$$
\begin{aligned}
H(x, \varphi)=\left(a _ { \square } ^ { 2 } \left(x+\sum_{j \in \mathbb{Z}_{+}} a_{j} x^{j+1}+\right.\right. & \left.\varphi \sum_{j \in \mathbb{Z}_{+}} n_{j} x^{j}\right), \\
& \left.a_{\square}\left(\sum_{j \in \mathbb{Z}_{+}} m_{j-\frac{1}{2}} x^{j}+\varphi\left(1+\sum_{j \in \mathbb{Z}_{+}} b_{j} x^{j}\right)\right)\right)
\end{aligned}
$$

with $a_{\square} \in\left(R^{0}\right)^{\times}$and $(a, m)=\left\{\left(a_{j}, m_{j-\frac{1}{2}}\right)\right\}_{j \in \mathbb{Z}_{+}}$, and satisfying $D \tilde{x}=\tilde{\varphi} D \tilde{\varphi}$, and formal power series of the form

$$
\exp \left(-\sum_{j \in \mathbb{Z}_{+}}\left(A_{j} L_{j}(x, \varphi)+M_{j-\frac{1}{2}} G_{j-\frac{1}{2}}(x, \varphi)\right)\right) \cdot\left(a_{\square}^{2} x, a_{\square} \varphi\right) .
$$

This defines a bijection

$$
\begin{aligned}
E: R^{\infty} & \longrightarrow R^{\infty} \\
(A, M)=\left\{\left(A_{j}, M_{j-\frac{1}{2}}\right)\right\}_{j \in \mathbb{Z}_{+}} & \mapsto \quad(a, m)=\left\{\left(a_{j}, m_{j-\frac{1}{2}}\right)\right\}_{j \in \mathbb{Z}_{+}} .
\end{aligned}
$$

As in [B4], this allows us to define a map $\tilde{E}$ from $R^{\infty}$ to the set of all formal superconformal power series in $x R[[x]][\varphi]$ with leading even coefficient of $\varphi$ equal 
to one, by defining

$$
\begin{aligned}
\varphi \tilde{E}^{0}(A, M)(x, \varphi) & =\varphi\left(x+\sum_{j \in \mathbb{Z}_{+}} E_{j}(A, M) x^{j+1}\right) \\
\varphi \tilde{E}^{1}(A, M)(x, \varphi) & =\varphi \sum_{j \in \mathbb{Z}_{+}} E_{j-\frac{1}{2}}(A, M) x^{j}
\end{aligned}
$$

and letting $\tilde{E}(A, M)(x, \varphi)$ be the unique formal superconformal power series with even coefficient of $\varphi$ equal to one such that the even and odd components of $\tilde{E}$ satisfy (2.6) and (2.7), respectively. For $a_{\square} \in\left(R^{0}\right)^{\times}$, we define a map $\hat{E}$ from $\left(R^{0}\right)^{\times} \times R^{\infty}$ to the set of all formal superconformal power series in $x R[[x]][\varphi]$ with invertible leading even coefficient of $\varphi$, by defining

$$
\begin{aligned}
\hat{E}\left(a_{\square}, A, M\right)(x, \varphi) & =\left(\hat{E}^{0}\left(a_{\square}, A, M\right)(x, \varphi), \hat{E}^{1}\left(a_{\square}, A, M\right)(x, \varphi)\right) \\
& =\left(a_{\square}^{2} \tilde{E}^{0}(A, M)(x, \varphi), a_{\square} \tilde{E}^{1}(A, M)(x, \varphi) .\right.
\end{aligned}
$$

The following proposition is proved in $\mathrm{B} 4$.

Proposition 2.1. (B⿺ superconformal power series $H(x, \varphi) \in x R[[x]][\varphi]$ of the form

$$
\varphi H(x, \varphi)=\varphi\left(a_{\square}^{2}\left(x+\sum_{j \in \mathbb{Z}_{+}} a_{j} x^{j+1}\right), a_{\square} \sum_{j \in \mathbb{Z}_{+}} m_{j-\frac{1}{2}} x^{j}\right)
$$

and with even coefficient of $\varphi$ equal to $a_{\square}$ for $\left(a_{\square}, a, m\right) \in\left(R^{0}\right)^{\times} \times R^{\infty}$, is a bijection.

The map $\tilde{E}$ from $R^{\infty}$ to the set of formal superconformal power series of the form (2.9) with $a_{\square}=1$ and even coefficient of $\varphi$ equal to 1 is also a bijection.

In particular, we have inverses $\tilde{E}^{-1}$ and $\hat{E}^{-1}$.

Let

$$
\begin{array}{r}
\mathcal{H}=\left\{(A, M) \in \bigwedge_{\infty}^{\infty} \mid \tilde{E}(A, M)(z, \theta)\right. \text { is an absolutely convergent power } \\
\text { series in some neighborhood of }(z, \theta)=0\},
\end{array}
$$

and for $n \in \mathbb{Z}_{+}$, let

$$
S M^{n-1}=\left\{\left(\left(z_{1}, \theta_{1}\right), \ldots,\left(z_{n-1}, \theta_{n-1}\right)\right) \mid\left(z_{i}, \theta_{i}\right) \in \bigwedge_{\infty}^{\times},\left(z_{i}\right)_{B} \neq\left(z_{j}\right)_{B}, \text { for } i \neq j\right\} .
$$

Note that for $n=1$, the set $S M^{0}$ has exactly one element.

For convenience, define the superconformal shift

$$
\begin{aligned}
s_{(z, \theta)}: \bigwedge_{\infty} & \rightarrow \bigwedge_{\infty} \\
(w, \rho) & \mapsto(w-z-\rho \theta, \rho-\theta) .
\end{aligned}
$$

We also define the linear operator $a_{\square}^{-2 L_{0}(x, \varphi)}=a_{\square}^{2 x \frac{\partial}{\partial x}+\varphi \frac{\partial}{\partial \varphi}}$ on $R\left[\left[x, x^{-1}\right]\right][\varphi]$, by

$$
a_{\square}^{-2 L_{0}(x, \varphi)} \cdot c \varphi^{k} x^{n}=c a_{\square}^{2 n+k} \varphi^{k} x^{n},
$$

for $c \in R, k=0,1$, and $n \in \mathbb{Z}$, and by extending linearly. 
For any supersphere with $1+n$ tubes over $\bigwedge_{\infty}$, we can write the power series expansion of the local coordinate at the $i$-th puncture $\left(z_{i}, \theta_{i}\right)$, for $i=1, \ldots, n$, as

$$
\begin{aligned}
H_{i}(w, \rho) & =\exp \left(-\sum_{j \in \mathbb{Z}_{+}}\left(A_{j}^{(i)} L_{j}(x, \varphi)+M_{j-\frac{1}{2}}^{(i)} G_{j-\frac{1}{2}}\right)\right) \cdot \\
& \left.\cdot\left(a_{\square}^{(i)}\right)^{-2 L_{0}(x, \varphi)} \cdot(x, \varphi)\right|_{(x, \varphi)=s_{\left(z_{i}, \theta_{i}\right)}(w, \rho)} \\
& =\hat{E}\left(a_{\square}^{(i)}, A^{(i)}, M^{(i)}\right) \circ s_{\left(z_{i}, \theta_{i}\right)}(w, \rho)
\end{aligned}
$$

for $\left(a_{\square}^{(i)}, A^{(i)}, M^{(i)}\right) \in\left(\bigwedge_{\infty}^{0}\right)^{\times} \times \mathcal{H}$, and we can write the power series expansion of the local coordinate at $\infty$ as

$$
\begin{aligned}
H_{0}(w, \rho) & =\exp \left(\sum_{j \in \mathbb{Z}_{+}}\left(A_{j}^{(0)} L_{-j}(w, \rho)+M_{j-\frac{1}{2}}^{(0)} G_{-j+\frac{1}{2}}(w, \rho)\right)\right) \cdot\left(\frac{1}{w}, \frac{i \rho}{w}\right) \\
& =\tilde{E}\left(A^{(0)},-i M^{(0)}\right)\left(\frac{1}{w}, \frac{i \rho}{w}\right)
\end{aligned}
$$

for $\left(A^{(0)},-i M^{(0)}\right) \in \mathcal{H}$. But $\left(A^{(0)},-i M^{(0)}\right) \in \mathcal{H}$ if and only if $\left(A^{(0)}, M^{(0)}\right) \in \mathcal{H}$. From $\mathrm{B} 4$, we have the following proposition.

Proposition 2.2. ([B] ) The moduli space of superspheres over $\bigwedge_{\infty}$ with $1+n$ tubes, for $n \in \mathbb{Z}_{+}$, can be identified with the set

$$
S K(n)=S M^{n-1} \times \mathcal{H} \times\left(\left(\bigwedge_{\infty}^{0}\right)^{\times} \times \mathcal{H}\right)^{n} .
$$

The moduli space of superspheres with one tube can be identified with the set

$$
S K(0)=\left\{(A, M) \in \mathcal{H} \mid\left(A_{1}, M_{\frac{1}{2}}\right)=(0,0)\right\} .
$$

Let $S K=\bigcup_{n \in \mathbb{N}} S K(n)$ denote the moduli space of superspheres over $\bigwedge_{\infty}$ with tubes. The actual elements of $S K$ give the data for a canonical supersphere representative of a given equivalence class of superspheres with tubes modulo superconformal equivalence. Any element of $S K(n)$, for $n \in \mathbb{Z}_{+}$, can be written as

$$
\left(\left(z_{1}, \theta_{1}\right), \ldots,\left(z_{n-1}, \theta_{n-1}\right) ;\left(A^{(0)}, M^{(0)}\right),\left(a_{\square}^{(1)}, A^{(1)}, M^{(1)}\right), \ldots,\left(a_{\square}^{(n)}, A^{(n)}, M^{(n)}\right)\right)
$$

where $\left(z_{1}, \theta_{1}\right), \ldots,\left(z_{n-1}, \theta_{n-1}\right) \in S M^{n-1},\left(A^{(0)}, M^{(0)}\right) \in \mathcal{H}$, and

$$
\left(a_{\square}^{(1)}, A^{(1)}, M^{(1)}\right), \ldots,\left(a_{\square}^{(n)}, A^{(n)}, M^{(n)}\right) \in\left(\bigwedge_{\infty}^{0}\right)^{\times} \times \mathcal{H} .
$$

Thus for an element $Q \in S K$, we can think of $Q$ as consisting of the above data, or as being a canonical supersphere with tubes corresponding to that data.

Note that above we are working over $\bigwedge_{\infty}$. But we can always restrict variables and coefficients to be in a finite dimensional Grassmann subalgebra of $\bigwedge_{\infty}$ if we so wish as long as care is taken to see that if we employ any partial derivatives with respect to odd variables that these partials are well defined.

2.2. A left action of the symmetric group $S_{n}$ on the moduli space $S K(n)$. Let $S_{n}$ be the group of permutations on $n$ letters, for $n \in \mathbb{Z}_{+}$. In B4], we defined an action of $S_{n}$ on $S K(n)$. We would like to take this opportunity to point out that although this action was stated to be a right action, it is in fact a left action. We define this left action by first defining an action of $S_{n-1}$ on $S K(n)$ given by permuting the ordering of the first $n-1$ positively oriented punctures and their 
local coordinates and then extending this to an action of $S_{n}$ on $S K(n)$, by defining the action of the transposition $(n-1 n)$ on $S K(n)$.

To define the action of $S_{n-1}$ on $S K(n)$, we note that an ordering or labeling of the first $n-1$ punctures of a supersphere with $n$-tubes with the first $n-1$ punctures at the points $p_{1}, p_{2}, \ldots, p_{n-1}$ is a bijection

$$
l:\{1,2, \ldots, n-1\} \longrightarrow\left\{p_{1}, p_{2}, \ldots, p_{n-1}\right\}
$$

Then a left action on any ordering $l$ is given by $\sigma \cdot l(i)=l\left(\sigma^{-1}(i)\right)$, for $\sigma \in S_{n-1}$ and $i=1, \ldots, n-1$. Thus, for $\sigma \in S_{n-1}$, and $Q \in S K(n)$ given by

$Q=\left(\left(z_{1}, \theta_{1}\right), \ldots,\left(z_{n-1}, \theta_{n-1}\right) ;\left(A^{(0)}, M^{(0)}\right),\left(a_{\square}^{(1)}, A^{(1)}, M^{(1)}\right), \ldots,\left(a_{\square}^{(n)}, A^{(n)}, M^{(n)}\right)\right)$,

$\sigma$ acting on $Q$, denoted $\sigma \cdot Q$, is given by

$$
\begin{array}{r}
\sigma \cdot Q=\left(\left(z_{\sigma^{-1}(1)}, \theta_{\sigma^{-1}(1)}\right), \ldots,\left(z_{\sigma^{-1}(n-1)}, \theta_{\sigma^{-1}(n-1)}\right) ;\left(A^{(0)}, M^{(0)}\right),\right. \\
\left(a_{\square}^{\left(\sigma^{-1}(1)\right)}, A^{\left(\sigma^{-1}(1)\right)}, M^{\left(\sigma^{-1}(1)\right)}\right), \ldots,\left(a_{\square}^{\left(\sigma^{-1}(n-1)\right)}, A^{\left(\sigma^{-1}(n-1)\right)}, M^{\left(\sigma^{-1}(n-1)\right)}\right) \\
\left.\left(a_{\square}^{(n)}, A^{(n)}, M^{(n)}\right)\right) .
\end{array}
$$

To extend this to a left action of $S_{n}$ on $S K(n)$, we first note that $S_{n}$ is generated by the symmetric group on the first $n-1$ letters $S_{n-1}$ and the transposition ( $n-$ $1 n)$. We can let $(n-1 n)$ act on $S K(n)$ by permuting the $(n-1)$-st and $n$ th punctures and their local coordinates for a canonical supersphere with $1+n$ tubes but the resulting supersphere with $1+n$ tubes is not canonical. To obtain the superconformally equivalent canonical supersphere, we have to translate the new $n$-th puncture to 0 . This translation will not change the local coordinates at positively oriented punctures but will change the local coordinates at the negatively oriented puncture $\infty$. In [B4] we show that the resulting canonical supersphere with tubes is given by

$$
\begin{aligned}
& \begin{array}{l}
n-1 n) \cdot Q \\
=( \\
\left(\infty,\left(z_{1}, \theta_{1}\right), \ldots,\left(z_{n-2}, \theta_{n-2}\right), 0,\left(z_{n-1}, \theta_{n-1}\right) ;\left(A^{(0)}, M^{(0)}\right),\left(a_{\square}^{(1)}, A^{(1)}, M^{(1)}\right),\right. \\
\left.\quad \ldots,\left(a_{\square}^{(n-2)}, A^{(n-2)}, M^{(n-2)}\right),\left(a_{\square}^{(n)}, A^{(n)}, M^{(n)}\right),\left(a_{\square}^{(n-1)}, A^{(n-1)}, M^{(n-1)}\right)\right) \\
=\left(\infty, s_{\left(z_{n-1}, \theta_{n-1}\right)}\left(z_{1}, \theta_{1}\right), s_{\left(z_{n-1}, \theta_{n-1}\right)}\left(z_{2}, \theta_{2}\right), \ldots, s_{\left(z_{n-1}, \theta_{n-1}\right)}\left(z_{n-2}, \theta_{n-2}\right),\right. \\
s_{\left(z_{n-1}, \theta_{n-1}\right)}(0), 0 ;\left(\tilde{A}^{(0)}, \tilde{M}^{(0)}\right),\left(a_{\square}^{(1)}, A^{(1)}, M^{(1)}\right), \ldots,\left(a_{\square}^{(n-2)}, A^{(n-2)}, M^{(n-2)}\right), \\
\left.\left(a_{\square}^{(n)}, A^{(n)}, M^{(n)}\right),\left(a_{\square}^{(n-1)}, A^{(n-1)}, M^{(n-1)}\right)\right) \in S K(n)
\end{array}
\end{aligned}
$$


where $\left(\tilde{A}^{(0)}, \tilde{M}^{(0)}\right) \in \bigwedge_{\infty}^{\infty}$ is given by

$$
\begin{aligned}
& \tilde{E}\left(\tilde{A}^{(0)},-i \tilde{M}^{(0)}\right)\left(\frac{1}{w}, \frac{i \rho}{w}\right) \\
= & \exp \left(\sum_{j \in \mathbb{Z}_{+}}\left(\tilde{A}_{j}^{(0)} L_{-j}(w, \rho)+\tilde{M}_{j-\frac{1}{2}}^{(0)} G_{-j+\frac{1}{2}}(w, \rho)\right)\right) \cdot\left(\frac{1}{w}, \frac{i \rho}{w}\right) \\
= & \exp \left(\sum_{j \in \mathbb{Z}_{+}}\left(A_{j}^{(0)} L_{-j}(x, \varphi)+M_{j-\frac{1}{2}}^{(0)} G_{-j+\frac{1}{2}}(x, \varphi)\right)\right) \cdot \\
= & \left.\exp \left(-\frac{1}{x}, \frac{i \varphi}{x}\right)\right|_{(x, \varphi)=s_{\left(-z_{n-1},-\theta_{n-1}\right)}(w, \rho)} \\
& \cdot \exp \left(\sum_{j \in \mathbb{Z}_{+}}\left(A_{j}^{(0)} L_{-j}(w, \rho)+M_{j-\frac{1}{2}}^{(0)} G_{-j+\frac{1}{2}}(w, \rho)\right)\right) \cdot\left(\frac{1}{w}, \frac{i \rho}{w}\right) .
\end{aligned}
$$

2.3. Supermeromorphic superfunctions on $S K$ and supermeromorphic tangent spaces of $S K$. A supermeromorphic superfunction on $S K(n)$, for $n \in$ $\mathbb{Z}_{+}$, is a superfunction $F: S K(n) \rightarrow \bigwedge_{\infty}$ of the form

$$
\begin{aligned}
& F(Q)= F\left(\left(z_{1}, \theta_{1}\right), \ldots,\left(z_{n-1}, \theta_{n-1}\right) ;\left(A^{(0)}, M^{(0)}\right),\left(a_{\square}^{(1)}, A^{(1)}, M^{(1)}\right), \ldots,\right. \\
&\left.\left(a_{\square}^{(n)}, A^{(n)}, M^{(n)}\right)\right) \\
&= F_{0}\left(\left(z_{1}, \theta_{1}\right), \ldots,\left(z_{n-1}, \theta_{n-1}\right) ;\left(A^{(0)}, M^{(0)}\right),\left(a_{\square}^{(1)}, A^{(1)}, M^{(1)}\right), \ldots,\right. \\
&\left.\left(a_{\square}^{(n)}, A^{(n)}, M^{(n)}\right)\right) \times\left(\prod_{i=1}^{n-1} z_{i}^{-s_{i}} \prod_{1 \leq i<j \leq n-1}\left(z_{i}-z_{j}-\theta_{i} \theta_{j}\right)^{-s_{i j}}\right)
\end{aligned}
$$

where $s_{i}$ and $s_{i j}$ are nonnegative integers and

$$
F_{0}\left(\left(z_{1}, \theta_{1}\right), \ldots,\left(z_{n-1}, \theta_{n-1}\right) ;\left(A^{(0)}, M^{(0)}\right),\left(a_{\square}^{(1)}, A^{(1)}, M^{(1)}\right), \ldots,\left(a_{\square}^{(n)}, A^{(n)}, M^{(n)}\right)\right)
$$

is a polynomial in the $z_{i}$ 's, $\theta_{i}$ 's, $a_{\square}^{(i)}$ 's, $\left(a_{\square}^{(i)}\right)^{-1}$ 's, $A_{j}^{(i)}$ 's, and $M_{j-1 / 2}^{(i)}$ 's. For $n=0$ a supermeromorphic superfunction on $S K(0)$ is a polynomial in the components of elements of $S K(0)$, i.e., a polynomial in the $A_{j}^{(0)}$ 's, and $M_{j-1 / 2}^{(0)}$ 's. For $F$ of the form (2.13), we say that $F$ has a pole of order $s_{i j}$ at $\left(z_{i}, \theta_{i}\right)=\left(z_{j}, \theta_{j}\right)$.

The set of all canonical supermeromorphic superfunctions on $S K(n)$ is denoted by $S D(n)$. Note that $S D(n)$ has a natural $\mathbb{Z}_{2}$-grading since any supermeromorphic superfunction $F$ can be decomposed into an even component $F^{0}$ and an odd component $F^{1}$ where $F^{0}(Q) \in \bigwedge_{\infty}^{0}$, for all $Q \in S K$ and $F^{1}(Q) \in \bigwedge_{\infty}^{1}$ for all $Q \in S K$.

An even supermeromorphic tangent vector of $S K$ at a point $Q \in S K$ is a linear map $\mathcal{L}_{Q}: S D(n) \longrightarrow \bigwedge_{\infty}$ such that for $F_{1}, F_{2} \in S D(n)$, the map $\mathcal{L}_{Q}$ satisfies $\mathcal{L}_{Q}\left(F_{1} F_{2}\right)=\mathcal{L}_{Q} F_{1} \cdot F_{2}(Q)+F_{1}(Q) \cdot \mathcal{L}_{Q} F_{2}$, and an odd supermeromorphic tangent vector of $S K$ at a point $Q \in S K$ is a linear map $\mathcal{L}_{Q}: S D(n) \longrightarrow \bigwedge_{\infty}$ such that for $F_{1}$ of homogeneous sign in $S D(n)$, we have $\mathcal{L}_{Q}\left(F_{1} F_{2}\right)=\mathcal{L}_{Q} F_{1} \cdot F_{2}(Q)+$ $(-1)^{\eta\left(F_{1}\right)} F_{1}(Q) \cdot \mathcal{L}_{Q} F_{2}$. The set of all supermeromorphic tangent vectors at $Q$ is the supermeromorphic tangent space of $S K$ at $Q$ and is denoted $T_{Q} S K$ (or $T_{Q} S K(n)$ when $Q \in S K(n))$. Since $T_{Q} S K$ is naturally $\mathbb{Z}_{2}$-graded, the sign function $\eta$ is well 
defined on $T_{Q} S K$. In [B2] and [B4, we prove that any supermeromorphic tangent vector $\mathcal{L}_{Q}$ can be expressed as

$$
\begin{aligned}
\mathcal{L}_{Q}=\sum_{i=1}^{n-1}\left(\left.\alpha_{i} \frac{\partial}{\partial z_{i}}\right|_{Q}+\left.\beta_{i} \frac{\partial}{\partial \theta_{i}}\right|_{Q}\right) & +\left.\sum_{i=1}^{n} \delta_{i} \frac{\partial}{\partial a_{\square}^{(i)}}\right|_{Q} \\
& +\sum_{i=0}^{n} \sum_{j \in \mathbb{Z}_{+}}\left(\left.\gamma_{i}^{j} \frac{\partial}{\partial A_{j}^{(i)}}\right|_{Q}+\left.\nu_{i}^{j} \frac{\partial}{\partial M_{j-\frac{1}{2}}^{(i)}}\right|_{Q}\right)
\end{aligned}
$$

where

$$
\alpha_{i}=\mathcal{L}_{Q} z_{i}, \quad \beta_{i}=\mathcal{L}_{Q} \theta_{i}, \quad \delta_{i}=\mathcal{L}_{Q} a_{\square}^{(i)}, \quad \gamma_{i}^{j}=\mathcal{L}_{Q} A_{j}^{(i)}, \quad \nu_{i}^{j}=\mathcal{L}_{Q} M_{j-\frac{1}{2}}^{(i)} .
$$

Of course if $\eta\left(\mathcal{L}_{Q}\right)=0$, then $\alpha_{i}, \delta_{i}, \gamma_{i}^{j} \in \bigwedge_{\infty}^{\eta\left(\mathcal{L}_{Q}\right)}$ and $\beta_{i}, \nu_{i}^{j} \in \bigwedge_{\infty}^{\left(\eta\left(\mathcal{L}_{Q}\right)+1\right) \bmod 2}$.

2.4. The sewing operation. In B4] we introduce the sewing operation for superspheres with tubes. This gives rise to the sewing equation, normalization conditions and boundary conditions derived from taking two canonical superspheres with tubes, sewing them together and determining conditions on the uniformizing function which maps the resulting supersphere to a super-Riemann sphere. This resulting super-Riemann sphere is then superconformal to a canonical supersphere, i.e., an element in $S K$, via a superprojective transformation. Thus for any $m \in \mathbb{Z}_{+}$, $n \in \mathbb{N}$, and any positive integer $i \leq m$, the sewing operation for superspheres with tubes defined in B4] induces an operation

$$
\begin{aligned}
{ }_{i} \infty_{0}: S K(m) \times S K(n) & \rightarrow S K(m+n-1) \\
\left(Q_{1}, Q_{2}\right) & \mapsto Q_{1}{ }_{i} \infty_{0} Q_{2} .
\end{aligned}
$$

For $m \in \mathbb{Z}_{+}$, let $Q_{1} \in S K(m)$ be given by

$$
\left(\left(z_{1}, \theta_{1}\right), \ldots,\left(z_{m-1}, \theta_{m-1}\right) ;\left(A^{(0)}, M^{(0)}\right),\left(a_{\square}^{(1)}, A^{(1)}, M^{(1)}\right), \ldots,\left(a_{\square}^{(m)}, A^{(m)}, M^{(m)}\right)\right)
$$

and for $n \in \mathbb{N}$, let $Q_{2} \in S K(n)$ be given by

$$
\left(\left(z_{1}^{\prime}, \theta_{1}^{\prime}\right), \ldots,\left(z_{n-1}^{\prime}, \theta_{n-1}^{\prime}\right) ;\left(B^{(0)}, N^{(0)}\right),\left(b_{\square}^{(1)}, B^{(1)}, N^{(1)}\right), \ldots,\left(b_{\square}^{(n)}, B^{(n)}, N^{(n)}\right)\right) .
$$

For convenience, we will sometimes denote the puncture at 0 of $Q_{1}$ by $\left(z_{m}, \theta_{m}\right)$. Let

$$
\begin{aligned}
H^{(1)}(w, \rho) & =\exp \left(-\sum_{j \in \mathbb{Z}_{+}}\left(A_{j}^{(i)} L_{j}(w, \rho)+M_{j-\frac{1}{2}}^{(i)} G_{j-\frac{1}{2}}(w, \rho)\right)\right) . \\
& =\hat{E}\left(a_{\square}^{(i)}, A^{(i)}, M^{(i)}\right)(w, \rho), \\
H^{(2)}(w, \rho) & =\exp \left(\sum_{j \in \mathbb{Z}_{+}}\left(B_{j}^{(i)}\right)^{-2 L_{0}(w, \rho)} \cdot(w, \rho)\right. \\
& =\tilde{E}\left(B_{-j}^{(0)},-i N^{(0)}\right)\left(\frac{1}{w}, \frac{i \rho}{w}\right) .
\end{aligned}
$$

Then the local coordinate vanishing at the $i$-th puncture of the canonical supersphere represented by $Q_{1}$ is $H^{(1)} \circ s_{\left(z_{i}, \theta_{i}\right)}(w, \rho)$, and the local coordinate vanishing at the puncture at $\infty$ of the canonical supersphere represented by $Q_{1}$ is $H^{(2)}(w, \rho)$. 
Recall the standard coordinate atlas for the super-Riemann sphere, $S \hat{\mathbb{C}}$, given by $\left\{\left(U_{\Delta}, \Delta\right),\left(U_{\Upsilon}, \Upsilon\right)\right\}$ with coordinate transition given by $I=\Delta \circ \Upsilon^{-1}$. For a superconformal equivalence $F: S \hat{\mathbb{C}} \rightarrow S \hat{\mathbb{C}}$ define $F_{\Delta}=\Delta \circ F \circ \Delta^{-1}$ and $F_{\Upsilon}=$ $\Upsilon \circ F \circ \Upsilon^{-1}$. Also define the DeWitt open (respectively, closed) discs about zero and of radius $r>0$, by

$$
\mathcal{B}_{0}^{r}=\left\{(w, \rho) \in \bigwedge_{\infty}|| w_{B} \mid<r\right\} \quad\left(\text { resp. }, \overline{\mathcal{B}}_{0}^{r}=\left\{(w, \rho) \in \bigwedge_{\infty}|| w_{B} \mid \leq r\right\}\right) .
$$

The following theorem summarizes several results from [B2] and [B4], and provides exact formulas for calculating the resulting canonical supersphere from the sewing together of two canonical superspheres.

Theorem 2.3. ([B] $)$ The $i$-th tube of $Q_{1}$ can be sewn with the 0-th tube of $Q_{2}$ if and only if there exist $r_{1}, r_{2} \in \mathbb{R}_{+}$, with $r_{1}>r_{2}$ such that the series $\left(H^{(1)}\right)^{-1}(w, \rho)$ and $\left(H^{(2)}\right)^{-1}(w, \rho)$ are absolutely convergent in $\mathcal{B}_{0}^{r_{1}}$ and $\mathcal{B}_{0}^{1 / r_{2}} \backslash\left(\{0\} \times\left(\bigwedge_{\infty}\right)_{S}\right)$, respectively, $\left(-z_{i},-\theta_{i}\right),\left(z_{k}-z_{i}-\theta_{k} \theta_{i}, \theta_{k}-\theta_{i}\right) \notin\left(H^{(1)}\right)^{-1}\left(\mathcal{B}_{0}^{r_{1}}\right)$, for $k=1, \ldots, m-1$, $k \neq i$, and $0,\left(z_{l}^{\prime}, \theta_{l}^{\prime}\right) \notin\left(H^{(2)}\right)^{-1}\left(\mathcal{B}_{0}^{1 / r_{2}} \backslash\left(\{0\} \times\left(\bigwedge_{\infty}\right)_{S}\right)\right)$, for $l=1, \ldots, n-1$. In this case, there exist unique bijective superconformal functions $F^{(1)}(w, \rho)$ and $F^{(2)}(w, \rho)$ defined on

and

$$
S \hat{\mathbb{C}} \backslash \Delta^{-1} \circ s_{\left(z_{i}, \theta_{i}\right)}^{-1} \circ\left(H^{(1)}\right)^{-1}\left(\overline{\mathcal{B}}_{0}^{r_{2}}\right)
$$

$$
U_{\Delta} \backslash \Delta^{-1} \circ\left(H^{(2)}\right)^{-1}\left(\overline{\mathcal{B}}_{0}^{1 / r_{1}} \backslash\left(\{0\} \times\left(\bigwedge_{\infty}\right)_{S}\right)\right) \subset S \hat{\mathbb{C}}
$$

respectively, satisfying the normalization conditions:

$$
\begin{aligned}
F_{\Upsilon}^{(1)}(0) & =0, \\
\lim _{w \rightarrow \infty} \frac{\partial}{\partial \rho}\left(F_{\Delta}^{(1)}\right)^{1}(w, \rho) & =1 \\
F_{\Delta}^{(2)}(0) & =0
\end{aligned}
$$

and such that in $\left(H^{(1)} \circ s_{\left(z_{i}, \theta_{i}\right)}\right)^{-1}\left(\mathcal{B}_{0}^{r_{1}} \backslash \overline{\mathcal{B}}_{0}^{r_{2}}\right)$, we have

$$
F_{\Delta}^{(1)}(w, \rho)=F_{\Delta}^{(2)} \circ\left(H^{(2)}\right)^{-1} \circ I \circ H^{(1)} \circ s_{\left(z_{i}, \theta_{i}\right)}(w, \rho) .
$$

Furthermore, if we let

$$
H_{0}^{(1)}(w, \rho)=\tilde{E}\left(A^{(0)},-i M^{(0)}\right)\left(\frac{1}{w}, \frac{i \rho}{w}\right)
$$

denote the local coordinate of the puncture vanishing at $\infty$ of the canonical supersphere represented by $Q_{1}$; let

$$
H_{k}^{(1)}(w, \rho)=\hat{E}\left(a_{\square}^{(k)}, A^{(k)}, M^{(k)}\right)(w, \rho),
$$

so that the local coordinate vanishing at the $k$-th puncture of the canonical supersphere represented by $Q_{1}$ is given by $H_{k}^{(1)} \circ s_{\left(z_{k}, \theta_{k}\right)}$, for $k=1, \ldots, m, k \neq i$; and let

$$
H_{l}^{(2)}(w, \rho)=\hat{E}\left(b_{\square}^{(l)}, B^{(l)}, N^{(l)}\right)(w, \rho),
$$

so that the local coordinate vanishing at the $l$-th puncture of the canonical supersphere represented by $Q_{2}$ is given by $H_{l}^{(2)} \circ s_{\left(z_{l}^{\prime}, \theta_{l}^{\prime}\right)}$, for $l=1, \ldots, n$; then we have the following:

(1) When $i=m$, and $n>0$, the punctures of the canonical supersphere represented by $Q_{1} m_{m} \infty_{0} Q_{2}$ are

$$
\infty, F_{\Delta}^{(1)}\left(z_{1}, \theta_{1}\right), \ldots, F_{\Delta}^{(1)}\left(z_{m-1}, \theta_{m-1}\right), F_{\Delta}^{(2)}\left(z_{1}^{\prime}, \theta_{1}^{\prime}\right), \ldots, F_{\Delta}^{(2)}\left(z_{n-1}^{\prime}, \theta_{n-1}^{\prime}\right), 0
$$


and the local coordinates vanishing at these punctures are

$$
\begin{gathered}
H_{0}^{(1)} \circ\left(F_{\Delta}^{(1)}\right)^{-1}(w, \rho), \\
H_{1}^{(1)} \circ s_{\left(z_{1}, \theta_{1}\right)} \circ\left(F_{\Delta}^{(1)}\right)^{-1}(w, \rho), \ldots, H_{m-1}^{(1)} \circ s_{\left(z_{m-1}, \theta_{m-1}\right)} \circ\left(F_{\Delta}^{(1)}\right)^{-1}(w, \rho), \\
H_{1}^{(2)} \circ s_{\left(z_{1}^{\prime}, \theta_{1}^{\prime}\right)} \circ\left(F_{\Delta}^{(2)}\right)^{-1}(w, \rho), \ldots, H_{n-1}^{(2)} \circ s_{\left(z_{n-1}^{\prime}, \theta_{n-1}^{\prime}\right)} \circ\left(F_{\Delta}^{(2)}\right)^{-1}(w, \rho) \\
H_{n}^{(2)} \circ\left(F_{\Delta}^{(2)}\right)^{-1}(w, \rho),
\end{gathered}
$$

respectively.

(2) When $i=m=1$ and $n=0$, the canonical supersphere represented by $Q_{1}{ }_{1} \infty_{0} Q_{2}$ has only the one puncture at $\infty$ with local coordinate given by

$$
H_{0}^{(1)} \circ\left(F_{\Delta}^{(1)}\right)^{-1} \circ s_{\left(a^{\prime}, m^{\prime}\right)}^{-1}(w, \rho),
$$

where $\left(a^{\prime}, m^{\prime}\right) \in \bigwedge_{\infty}$ is the unique element such that $Q_{1}{ }_{1} \infty_{0} Q_{2}$ represents a canonical supersphere in $S K(0)$, i.e., such that the expansion of (2.19) has even coefficient of $w^{-2}$ and odd coefficient of $w^{-1}$ equal to zero. Thus

$$
\begin{aligned}
&\left.Q_{1{ }_{1} \infty_{0} Q_{2}=\left(( \tilde { E } ^ { - 1 } ) ^ { 0 } \left(H_{0}^{(1)} \circ\right.\right.}\left(F_{\Delta}^{(1)}\right)^{-1} \circ s_{\left(a^{\prime}, m^{\prime}\right)}^{-1} \circ I^{-1}(w, \rho)\right), \\
&\left.i\left(\tilde{E}^{-1}\right)^{1}\left(H_{0}^{(1)} \circ\left(F_{\Delta}^{(1)}\right)^{-1} \circ s_{\left(a^{\prime}, m^{\prime}\right)}^{-1} \circ I^{-1}(w, \rho)\right)\right) .
\end{aligned}
$$

(3) When $i=m>1$, and $n=0$, and writing $F_{\Delta}^{(1)}\left(z_{m-1}, \theta_{m-1}\right)=p \in \Lambda_{\infty}$, the punctures of the canonical supersphere represented by $Q_{1}{ }_{i} \infty_{0} Q_{2}$ are

$$
\infty, s_{p} \circ F_{\Delta}^{(1)}\left(z_{1}, \theta_{1}\right), \ldots, s_{p} \circ F_{\Delta}^{(1)}\left(z_{m-2}, \theta_{m-2}\right), 0 ;
$$

and the local coordinates vanishing at these punctures are

$$
\begin{gathered}
H_{0}^{(1)} \circ\left(F_{\Delta}^{(1)}\right)^{-1} \circ s_{p}^{-1}(w, \rho), \\
H_{1}^{(1)} \circ s_{\left(z_{1}, \theta_{1}\right)} \circ\left(F_{\Delta}^{(1)}\right)^{-1} \circ s_{p}^{-1}(w, \rho), \ldots, H_{m-2}^{(1)} \circ s_{\left(z_{m-2}, \theta_{m-2}\right)} \circ\left(F_{\Delta}^{(1)}\right)^{-1} \circ s_{p}^{-1}(w, \rho) \\
H_{m-1}^{(1)} \circ s_{\left(z_{m-1}, \theta_{m-1}\right)} \circ\left(F_{\Delta}^{(1)}\right)^{-1} \circ s_{p}^{-1}(w, \rho),
\end{gathered}
$$

respectively.

(4) When $i<m$ and $n \neq 0$, writing $F_{\Delta}^{(1)}(0)=p \in \Lambda_{\infty}$, the punctures of the canonical supersphere represented by $Q_{1}{ }_{i} \infty_{0} Q_{2}$ are

$$
\begin{gathered}
\infty, s_{p} \circ F_{\Delta}^{(1)}\left(z_{1}, \theta_{1}\right), \ldots, s_{p} \circ F_{\Delta}^{(1)}\left(z_{i-1}, \theta_{i-1}\right), s_{p} \circ F_{\Delta}^{(2)}\left(z_{1}^{\prime}, \theta_{1}^{\prime}\right), \ldots, \\
s_{p} \circ F_{\Delta}^{(2)}\left(z_{n-1}^{\prime}, \theta_{n-1}^{\prime}\right),-F_{\Delta}^{(1)}(0), s_{p} \circ F_{\Delta}^{(1)}\left(z_{i+1}, \theta_{i+1}\right), \ldots, \\
s_{p} \circ F_{\Delta}^{(1)}\left(z_{m-1}, \theta_{m-1}\right), 0 ;
\end{gathered}
$$

and the local coordinates vanishing at these punctures are

$$
\begin{gathered}
H_{0}^{(1)} \circ\left(F_{\Delta}^{(1)}\right)^{-1} \circ s_{p}^{-1}(w, \rho), \\
H_{1}^{(1)} \circ s_{\left(z_{1}, \theta_{1}\right)} \circ\left(F_{\Delta}^{(1)}\right)^{-1} \circ s_{p}^{-1}(w, \rho), \ldots, H_{i-1}^{(1)} \circ s_{\left(z_{i-1}, \theta_{i-1}\right)} \circ\left(F_{\Delta}^{(1)}\right)^{-1} \circ s_{p}^{-1}(w, \rho), \\
H_{1}^{(2)} \circ s_{\left(z_{1}^{\prime}, \theta_{1}^{\prime}\right)} \circ\left(F_{\Delta}^{(2)}\right)^{-1} \circ s_{p}^{-1}(w, \rho), \ldots, H_{n-1}^{(2)} \circ s_{\left(z_{n-1}^{\prime}, \theta_{n-1}^{\prime}\right)} \circ\left(F_{\Delta}^{(2)}\right)^{-1} \circ s_{p}^{-1}(w, \rho), \\
H_{n}^{(2)} \circ\left(F_{\Delta}^{(2)}\right)^{-1} \circ s_{p}^{-1}(w, \rho), \\
H_{i+1}^{(1)} \circ s_{\left(z_{i+1}, \theta_{i+1}\right)} \circ\left(F_{\Delta}^{(1)}\right)^{-1} \circ s_{p}^{-1}(w, \rho), \ldots, \\
H_{m-1}^{(1)} \circ s_{\left(z_{m-1}, \theta_{m-1}\right)} \circ\left(F_{\Delta}^{(1)}\right)^{-1} \circ s_{p}^{-1}(w, \rho),
\end{gathered}
$$


respectively.

$$
H_{m}^{(1)} \circ\left(F_{\Delta}^{(1)}\right)^{-1} \circ s_{p}^{-1}(w, \rho)
$$

(5) When $i<m$ and $n=0$, writing $F_{\Delta}^{(1)}(0)=p \in \Lambda_{\infty}$, the punctures of the canonical supersphere represented by $Q_{1}{ }_{i} \infty_{0} Q_{2}$ are

$$
\begin{aligned}
\infty, s_{p} \circ F_{\Delta}^{(1)}\left(z_{1}, \theta_{1}\right), \ldots, s_{p} \circ F_{\Delta}^{(1)}\left(z_{i-1}, \theta_{i-1}\right), s_{p} \circ F_{\Delta}^{(1)}\left(z_{i+1}, \theta_{i+1}\right), \ldots, \\
s_{p} \circ F_{\Delta}^{(1)}\left(z_{m-1}, \theta_{m-1}\right), 0 ;
\end{aligned}
$$

and the local coordinates vanishing at these punctures are

$$
\begin{gathered}
H_{0}^{(1)} \circ\left(F_{\Delta}^{(1)}\right)^{-1} \circ s_{p}^{-1}(w, \rho), \\
H_{1}^{(1)} \circ s_{\left(z_{1}, \theta_{1}\right)} \circ\left(F_{\Delta}^{(1)}\right)^{-1} \circ s_{p}^{-1}(w, \rho), \ldots, H_{i-1}^{(1)} \circ s_{\left(z_{i-1}, \theta_{i-1}\right)} \circ\left(F_{\Delta}^{(1)}\right)^{-1} \circ s_{p}^{-1}(w, \rho), \\
H_{i+1}^{(1)} \circ s_{\left(z_{i+1}, \theta_{i+1}\right)} \circ\left(F_{\Delta}^{(1)}\right)^{-1} \circ s_{p}^{-1}(w, \rho), \ldots, \\
H_{m-1}^{(1)} \circ s_{\left(z_{m-1}, \theta_{m-1}\right)} \circ\left(F_{\Delta}^{(1)}\right)^{-1} \circ s_{p}^{-1}(w, \rho), \\
H_{m}^{(1)} \circ\left(F_{\Delta}^{(1)}\right)^{-1} \circ s_{p}^{-1}(w, \rho),
\end{gathered}
$$

respectively.

Moreover, the components $F_{\Delta}^{(1)}$ and $F_{\Delta}^{(2)}$ of the uniformizing function can be expanded in formal power series such that

$$
\begin{aligned}
& F_{\Delta}^{(1)}(x, \varphi)=\left.\bar{F}^{(1)} \circ s_{\left(z_{i}, \theta_{i}\right)}(x, \varphi)\right|_{\left(\alpha_{0}^{1 / 2}, \mathcal{A}, \mathcal{M}, \mathcal{B}, \mathcal{N}\right)=\left(a_{\square}^{(i)}, A^{(i)}, M^{(i)}, B^{(0)}, N^{(0)}\right)} \\
& F_{\Delta}^{(2)}(x, \varphi)=\left.\bar{F}^{(2)}(x, \varphi)\right|_{\left(\alpha_{0}^{1 / 2}, \mathcal{A}, \mathcal{M}, \mathcal{B}, \mathcal{N}\right)=\left(a_{\square}^{(i)}, A^{(i)}, M^{(i)}, B^{(0)}, N^{(0)}\right)},
\end{aligned}
$$

where

$$
\begin{aligned}
\bar{F}^{(1)}(x, \varphi)= & \exp \left(\sum_{j \in \mathbb{Z}_{+}}\left(\Psi_{-j} L_{-j}(x, \varphi)+\Psi_{-j+\frac{1}{2}} G_{-j+\frac{1}{2}}(x, \varphi)\right)\right) \cdot(x, \varphi) \\
\bar{F}^{(2)}(x, \varphi)= & \exp \left(-\Psi_{0} 2 L_{0}(x, \varphi)\right) \cdot\left(a_{\square}^{(i)}\right)^{2 L_{0}(x, \varphi)} . \\
& \quad \exp \left(-\sum_{j \in \mathbb{Z}_{+}}\left(\Psi_{j} L_{j}(x, \varphi)+\Psi_{j-\frac{1}{2}} G_{j-\frac{1}{2}}(x, \varphi)\right)\right) \cdot(x, \varphi)
\end{aligned}
$$

are formal power series in

$x \mathbb{C}\left[\alpha_{0}^{\frac{1}{2}}, \alpha_{0}^{-\frac{1}{2}}\right][[\mathcal{A}, \mathcal{B}]][\mathcal{M}, \mathcal{N}]\left[\left[x^{-1}\right]\right][\varphi]$ and $x \mathbb{C}\left[\alpha_{0}^{\frac{1}{2}}, \alpha_{0}^{-\frac{1}{2}}\right][[\mathcal{A}, \mathcal{B}]][\mathcal{M}, \mathcal{N}][[x]][\varphi]$, respectively, and where

$$
\left(\Psi_{j}, \Psi_{j-\frac{1}{2}}\right)=\left(\Psi_{j}, \Psi_{j-\frac{1}{2}}\right)\left(\alpha_{0}^{\frac{1}{2}}, \mathcal{A}, \mathcal{M}, \mathcal{B}, \mathcal{N}\right)
$$

are a unique pair of sequences in $\mathbb{C}\left[\alpha_{0}^{1 / 2}, \alpha_{0}^{-1 / 2}\right][[\mathcal{A}, \mathcal{B}]][\mathcal{M}, \mathcal{N}]$, for $j \in \mathbb{Z}$, such that

$$
\begin{aligned}
\left(\Psi_{j}, \Psi_{j-\frac{1}{2}}\right) & =\left(-\mathcal{A}_{j},-\mathcal{M}_{j-\frac{1}{2}}\right)+\mathcal{P}_{j}\left(\alpha_{0}^{\frac{1}{2}}, \mathcal{A}, \mathcal{M}, \mathcal{B}, \mathcal{N}\right), \\
\left(\Psi_{-j}, \Psi_{-j+\frac{1}{2}}\right) & =\left(-\alpha_{0}^{-j} \mathcal{B}_{j},-\alpha_{0}^{-j+\frac{1}{2}} \mathcal{N}_{j-\frac{1}{2}}\right)+\mathcal{P}_{-j}\left(\alpha_{0}^{\frac{1}{2}}, \mathcal{A}, \mathcal{M}, \mathcal{B}, \mathcal{N}\right), \\
\Psi_{0} & =0+\mathcal{P}_{0}\left(\alpha_{0}^{\frac{1}{2}}, \mathcal{A}, \mathcal{M}, \mathcal{B}, \mathcal{N}\right),
\end{aligned}
$$


each $\mathcal{P}_{j}\left(\alpha_{0}^{1 / 2}, \mathcal{A}, \mathcal{M}, \mathcal{B}, \mathcal{N}\right)$, for $j \in \mathbb{Z}$, contains only terms with total degree at least one in the $\mathcal{A}_{k}$ 's and $\mathcal{M}_{k-1 / 2}$ 's, for $k \in \mathbb{Z}_{+}$, and with total degree at least one in the $\mathcal{B}_{k}$ 's and $\mathcal{N}_{k-1 / 2}$ 's, for $k \in \mathbb{Z}_{+}$.

In particular, letting $t^{1 / 2}$ be a complex variable, and letting

$$
\Psi_{j}\left(t^{\frac{1}{2}}\right)=\Psi_{j}\left(t^{-\frac{1}{2}} a_{\square}^{(i)}, A^{(i)}, M^{(i)}, B^{(0)}, N^{(0)}\right),
$$

for $j \in \frac{1}{2} \mathbb{Z}$. Then $\Psi_{j}\left(t^{1 / 2}\right) \in \bigwedge_{\infty}\left[\left[t^{-1 / 2}, t^{1 / 2}\right]\right]$ and if $Q_{1}{ }_{i} \infty_{0} Q_{2}$ exists, then the series $\Psi_{j}\left(t^{1 / 2}\right)$, for $j \in \frac{1}{2} \mathbb{Z}$, are convergent when $\left|t^{1 / 2}\right| \leq 1$, and the values of these convergent series are equal to the coefficients $\Psi_{j}$ of the power series (2.20) and 2.21).

Finally, using this solution of the uniformizing function $F$ given by $F^{(1)}$ and $F^{(2)}$, we obtain the canonical element of $S K(m+n-1)$ representing the sewn spheres by taking $\hat{E}^{-1}$ of each of the local coordinates given in (1) - (5).

Remark 2.4. The moduli space of $N=1$ superspheres with tubes, $S K$, along with the sewing operation and the action of the symmetric group defined in Section 2.2 is an example of a partial operad (cf. [M], [HL1, [HL2, [H2]).

The element of $\mathcal{H}$ with all components equal to 0 will be denoted by $(\mathbf{0}, \mathbf{0})$ or just $\mathbf{0}$. Note that the local coordinate chart corresponding to $(1, \mathbf{0}, \mathbf{0}) \in\left(\bigwedge_{\infty}^{0}\right)^{\times} \times \mathcal{H}$ is the identity map on $\bigwedge_{\infty}$ if the puncture is at 0 ; the shift $s_{\left(z_{i}, \theta_{i}\right)}(w, \rho)=\left(w-z_{i}-\right.$ $\left.\rho \theta_{i}, \rho-\theta_{i}\right)$ if the puncture is at $\left(z_{i}, \theta_{i}\right)$; and $I(w, \rho)=(1 / w, i \rho / w)$ if the puncture is at $\infty$. We will sometimes refer to such coordinates as standard local coordinates. From Theorem 2.3 we see that $S K$ has a unit under the sewing operation given by $e=(\mathbf{0},(1, \mathbf{0})) \in S K(1)$, i.e., for $Q \in S K(n)$ and $0<i \leq n$, the $i$-th puncture of $Q$ can always be sewn with the 0 -th puncture of $e$, the first puncture of $e$ can always be sewn with the 0 -th puncture of $Q$, and we have $Q_{i} \infty_{0} e=Q=e_{1} \infty_{0} Q$. From the geometry of sewing defined in [B4], the following associativity of the sewing operation is obvious.

Proposition 2.5. (B4) Let $l \in \mathbb{Z}_{+}$and $m, n \in \mathbb{N}$ such that $l+m-1 \in \mathbb{Z}_{+}$, and let $Q_{1} \in S K(l), Q_{2} \in S K(m), Q_{3} \in S K(n)$, and $i, j \in \mathbb{Z}_{+}$such that $1 \leq i \leq l$, and $1 \leq j \leq l+m-1$. The iterated sewings $\left(Q_{1} i_{i} \infty_{0} Q_{2}\right)_{j} \infty_{0} Q_{3}$ exist if and only if one of the following holds:

(i) $j<i$ and the sewings $\left(Q_{1}{ }_{j} \infty_{0} Q_{3}\right)_{i+n-1} \infty_{0} Q_{2}$ exist, in which case

$$
\left(Q_{1}{ }_{i} \infty_{0} Q_{2}\right)_{j} \infty_{0} Q_{3}=\left(Q_{1}{ }_{j} \infty_{0} Q_{3}\right)_{i+n-1} \infty_{0} Q_{2} ;
$$

(ii) $j \geq i+m$ and the sewings $\left(Q_{1}{ }_{j-m+1} \infty_{0} Q_{3}\right)_{i} \infty_{0} Q_{2}$ exist, in which case

$$
\left(Q_{1}{ }_{i} \infty_{0} Q_{2}\right)_{j} \infty_{0} Q_{3}=\left(Q_{1 j-m+1} \infty_{0} Q_{3}\right)_{i} \infty_{0} Q_{2} ;
$$

or

(iii) $i \leq j<i+m$ and the sewings $Q_{1}{ }_{i} \infty_{0}\left(Q_{2}{ }_{j-i+1} \infty_{0} Q_{3}\right)$ exist, in which case

$$
\left(Q_{1 i} \infty_{0} Q_{2}\right)_{j} \infty_{0} Q_{3}=Q_{1 i} \infty_{0}\left(Q_{2 j-i+1} \infty_{0} Q_{3}\right) .
$$

\subsection{Superspheres with one, two, and three tubes.}

Proposition 2.6. (B⿺) Any element $Q \in S K$ can be obtained by sewing the following types of elements of $S K$

(i) $(\mathbf{0}) \in S K(0)$,

(ii) $\left(\left(A^{(0)}, M^{(0)}\right),\left(a_{\square}^{(1)}, A^{(1)}, M^{(1)}\right)\right) \in S K(1)$, 
(iii) $((z, \theta) ; \mathbf{0},(1, \mathbf{0}),(1, \mathbf{0})) \in S K(2)$.

It is clear from the definition of sewing that $S K(1)$ is a partial monoid. In the following proposition, we give some subgroups of $S K(1)$.

Proposition 2.7. ([B] $)$ Let $s, t \in \Lambda_{\infty}^{0}$, and $(A, M) \in \Lambda_{\infty}^{\infty}$. Assume

$$
s(A, M), t(A, M) \in \mathcal{H} .
$$

Then $(s+t)(A, M) \in \mathcal{H}$, both

$$
\left(\mathbf{0},(1, t(A, M)){ }_{1} \infty_{0}(\mathbf{0},(1, s(A, M))\right.
$$

and

$$
(s(A, M),(1, \mathbf{0})){ }_{1} \infty_{0}(t(A, M),(1, \mathbf{0}))
$$

exist, and we have

$$
\begin{aligned}
& (\mathbf{0},(1,(s+t)(A, M)))=\left(\mathbf{0},(1, t(A, M)){ }_{1} \infty_{0}(\mathbf{0},(1, s(A, M))\right. \\
& ((s+t)(A, M),(1, \mathbf{0}))=(s(A, M),(1, \mathbf{0})){ }_{1} \infty_{0}(t(A, M),(1, \mathbf{0})) .
\end{aligned}
$$

In particular, for $(A, M) \in \mathcal{H}$, the sets

$$
\left\{\left(\mathbf{0},(1, t(A, M)) \mid t \in \bigwedge_{\infty}^{0}, t(A, M) \in \mathcal{H}\right\}\right.
$$

and

$$
\left\{(t(A, M),(1, \mathbf{0})) \mid t \in \bigwedge_{\infty}^{0}, t(A, M) \in \mathcal{H}\right\}
$$

are subgroups of $S K(1)$. In addition we have the subgroups given by taking $A=\mathbf{0}$ or $M=\mathbf{0}$ in 2.30) and 2.31).

Proposition 2.8. ([B4]) Let $z_{1}, z_{2} \in \bigwedge_{\infty}^{0}$ such that $\left|\left(z_{1}\right)_{B}\right|>\left|\left(z_{2}\right)_{B}\right|>\mid\left(z_{1}\right)_{B}-$ $\left(z_{2}\right)_{B} \mid>0$. Then

$$
\begin{aligned}
& \left(\left(z_{1}, \theta_{1}\right),\left(z_{2}, \theta_{2}\right) ; \mathbf{0},(1, \mathbf{0}),(1, \mathbf{0}),(1, \mathbf{0})\right)= \\
& \quad=\left(\left(z_{2}, \theta_{2}\right) ; \mathbf{0},(1, \mathbf{0}),(1, \mathbf{0})\right){ }_{1} \infty_{0}\left(\left(z_{1}-z_{2}-\theta_{1} \theta_{2}, \theta_{1}-\theta_{2}\right) ;\right. \\
& \quad \mathbf{0},(1, \mathbf{0}),(1, \mathbf{0})) \\
& =\left(\left(z_{1}, \theta_{1}\right) ; \mathbf{0},(1, \mathbf{0}),(1, \mathbf{0})\right){ }_{2} \infty_{0}\left(\left(z_{2}, \theta_{2}\right) ; \mathbf{0},(1, \mathbf{0}),(1, \mathbf{0})\right) .
\end{aligned}
$$

Remark 2.9. Proposition 2.8 is used in Section 6 to prove the associativity property for an $N=1$ Neveu-Schwarz vertex operator superalgebra with odd formal variables obtained from an $N=1$ supergeometric vertex operator superalgebra. Thus Proposition 2.8 can be thought of as a geometric version of this algebraic relation.

Let $(a, m) \in \bigwedge_{\infty}^{\infty}$, and $k, l \in \mathbb{Z}_{+}$, and define

$$
\begin{gathered}
(A(a, k), M(m, l-1 / 2))=\left(\left\{A_{j} \mid A_{k}=a, A_{j}=0, \text { for } j \neq k\right\}_{j \in \mathbb{Z}_{+}},\right. \\
\left.\left\{M_{j-\frac{1}{2}} \mid M_{l-\frac{1}{2}}=m, M_{j-\frac{1}{2}}=0, \text { for } j \neq l\right\}_{j \in \mathbb{Z}_{+}}\right)
\end{gathered}
$$

which is an element of $\bigwedge_{\infty}^{\infty}$. 
Proposition 2.10. ([B] $)$ For $(z, \theta),\left(z_{0}, \theta_{0}\right) \in \bigwedge_{\infty}$ such that $0<\left|\left(z_{0}\right)_{B}\right|<\left|z_{B}\right|$, we have

$$
\begin{aligned}
& \left(\left(z_{0}+z+\theta_{0} \theta, \theta+\theta_{0}\right) ; \mathbf{0},(1, \mathbf{0}),(1, \mathbf{0})\right)= \\
& ((z, \theta) ; \mathbf{0},(1, \mathbf{0}),(1, \mathbf{0})){ }_{1} \infty_{0}\left(\left(A\left(-z_{0}, 1\right), M\left(-\theta_{0}, 1 / 2\right)\right),(1, \mathbf{0})\right) .
\end{aligned}
$$

Let $\epsilon \in \bigwedge_{\infty}^{1}$. From the definition of $S K(0)$, we see that $(\mathbf{0}, M(\epsilon, 3 / 2)) \in S K(0)$. Let $F$ be any element of $S D(1)$, and $(z, \theta) \in \bigwedge_{\infty}$. We define a linear functional on $S D(1)$ by

$$
\mathcal{G}_{e}(z, \theta) F=\left.\frac{d}{d \epsilon} F\left(((z, \theta) ; \mathbf{0},(1, \mathbf{0}),(1, \mathbf{0})){ }_{1} \infty_{0}(\mathbf{0}, M(\epsilon, 3 / 2))\right)\right|_{\epsilon=0} .
$$

Proposition 2.11. (B⿺辶) The linear functional $\mathcal{G}_{e}(z, \theta)$ is in $T_{e} S K(1)$, and

$$
\begin{aligned}
\mathcal{G}_{e}(z, \theta)=\sum_{k=0}^{1} \sum_{j \in \mathbb{Z}_{+}} & \left.z^{-(2 k-1) j-2+k} \frac{\partial}{\partial M_{j-\frac{1}{2}}^{(k)}}\right|_{e} \\
& +2 \theta\left(\left.z^{-2} \frac{1}{2} \frac{\partial}{\partial a_{\square}^{(1)}}\right|_{e}+\left.\sum_{k=0}^{1} \sum_{j \in \mathbb{Z}_{+}} z^{-(2 k-1) j-2} \frac{\partial}{\partial A_{j}^{(k)}}\right|_{e}\right) .
\end{aligned}
$$

2.6. An $N=1$ Neveu-Schwarz algebra structure of central charge zero on the supermeromorphic tangent space of $S K(1)$ at its identity. In B4] we define a bracket operation on a subspace $\hat{T}_{e} S K(1)$ of the supermeromorphic tangent space $T_{e} S K(1)$ of $S K(1)$ at the identity $e$. This subspace $\hat{T}_{e} S K(1)$ is the subspace of $T_{e} S K(1)$ consisting of all finite linear combinations of $-\left.\frac{\partial}{\partial a}\right|_{e},-\left.\frac{\partial}{\partial A_{j}^{(0)}}\right|_{e}$, $-\left.\frac{\partial}{\partial M_{j-1 / 2}^{(0)}}\right|_{e},-\left.\frac{\partial}{\partial A_{j}^{(1)}}\right|_{e}$, and $-\left.\frac{\partial}{\partial M_{j-1 / 2}^{(1)}}\right|_{e}$, for $j \in \mathbb{Z}_{+}$. In [B4], we prove the following proposition.

Proposition 2.12. (B4]) The vector space $\hat{T}_{e} S K(1)$ with the bracket operation defined in [B4 is the $N=1$ Neveu-Schwarz algebra with central charge zero. The basis is given by

$$
\begin{aligned}
\mathcal{L}(j) & =-\left.\frac{\partial}{\partial A_{-j}^{(0)}}\right|_{e}, \quad \text { for }-j \in \mathbb{Z}_{+}, \\
\mathcal{L}(j) & =-\left.\frac{\partial}{\partial A_{j}^{(1)}}\right|_{e}, \quad \text { for } j \in \mathbb{Z}_{+}, \\
\mathcal{L}(0) & =-\left.\frac{1}{2} \frac{\partial}{\partial a}\right|_{e}, \\
\mathcal{G}\left(j+\frac{1}{2}\right) & =-\left.\frac{\partial}{\partial M_{-j-\frac{1}{2}}^{(0)}}\right|_{e}, \quad \text { for }-j \in \mathbb{Z}_{+}, \\
\mathcal{G}\left(j-\frac{1}{2}\right) & =-\left.\frac{\partial}{\partial M_{j-\frac{1}{2}}^{(1)}}\right|_{e}, \text { for } j \in \mathbb{Z}_{+} .
\end{aligned}
$$




\section{The SEWING IDENTities on MOdules for the $N=1$ Neveu-Schwarz}

\section{ALGEBRA}

In this section we recall from [B4 the sewing identities on modules for the $N=1$ Neveu-Schwarz algebra, $\mathfrak{n s}$, arising from the sewing operation on the moduli space of superspheres with tubes.

3.1. Modules for the $\mathfrak{n}$ s. For any representation of $\mathfrak{n} \mathfrak{s}$, we shall use $L(j), G(j-$ $1 / 2)$ and $c \in \mathbb{C}$ to denote the representation images of $L_{j}, G_{j-1 / 2}$ and $d$, respectively.

Let $V=\bigsqcup_{k \in(1 / 2) \mathbb{Z}} V_{(k)}$ be a module for $\mathfrak{n s}$ of central charge $c \in \mathbb{C}$ (i.e., $d v=$ $c v$ for $v \in V)$ such that for $v \in V_{(k)}$, we have $L(0) v=k v$. Let $P(k)$ be the projection from $V$ to $V_{(k)}$. For any formal variable $t^{1 / 2}$ and $l \in 2 \mathbb{Z} \backslash\{0\}$, we define $\left(t^{1 / 2}\right)^{l L(0)} \in($ End $V)\left[\left[t^{1 / 2}, t^{-1 / 2}\right]\right]$ by $\left(t^{1 / 2}\right)^{l L(0)} v=\left(t^{1 / 2}\right)^{k l} v$ for $v \in V_{(k)}$, or equivalently $\left(t^{1 / 2}\right)^{l L(0)} v=\sum_{k \in(1 / 2) \mathbb{Z}} P(k)\left(t^{1 / 2}\right)^{k l} v$ for any $v \in V$.

Let $V_{P}$ be a vector space over $\mathbb{C}$ with basis $\left\{P_{k} \mid k \in(1 / 2) \mathbb{Z}\right\}$. Let $T\left(\mathfrak{n} \mathfrak{s} \oplus V_{P}\right)$ be the tensor algebra generated by the direct sum of $\mathfrak{n s}$ and $V_{P}$, and let $\mathcal{I}$ be the ideal of $T\left(\mathfrak{n} \mathfrak{s} \oplus V_{P}\right)$ generated by

$$
\begin{gathered}
\left\{L_{m} \otimes L_{n}-L_{n} \otimes L_{m}-\left[L_{m}, L_{n}\right], L_{m} \otimes G_{n-\frac{1}{2}}-G_{n-\frac{1}{2}} \otimes L_{m}-\left[L_{m}, G_{n-\frac{1}{2}}\right],\right. \\
G_{m+\frac{1}{2}} \otimes G_{n-\frac{1}{2}}+G_{n-\frac{1}{2}} \otimes G_{m+\frac{1}{2}}-\left[G_{m+\frac{1}{2}}, G_{n-\frac{1}{2}}\right], L_{n} \otimes d-d \otimes L_{n}, \\
G_{n-\frac{1}{2}} \otimes d-d \otimes G_{n-\frac{1}{2}}, P_{j} \otimes P_{k}-\delta_{j, k} P_{j}, P_{j} \otimes L_{n}-L_{n} \otimes P_{j+n}, \\
\left.P_{j} \otimes G_{n-\frac{1}{2}}-G_{n-\frac{1}{2}} \otimes P_{j+n-\frac{1}{2}}, P_{j} \otimes d-d \otimes P_{j} \mid m, n \in \mathbb{Z}, j, k \in \frac{1}{2} \mathbb{Z}\right\} .
\end{gathered}
$$

Then $U_{P}(\mathfrak{n} \mathfrak{s})=T\left(\mathfrak{n} \mathfrak{s} \oplus V_{P}\right) / \mathcal{I}$ is an associative superalgebra and the universal enveloping algebra $U(\mathfrak{n} \mathfrak{s})$ of $\mathfrak{n} \mathfrak{s}$ is a subalgebra. Linearly $U_{P}(\mathfrak{n} \mathfrak{s})=U(\mathfrak{n} \mathfrak{s}) \otimes V_{P}$.

For any even formal variable $t^{1 / 2}$ and $l \in 2 \mathbb{Z} \backslash\{0\}$, we define

$$
\left(t^{\frac{1}{2}}\right)^{l L_{0}}=\sum_{k \in \frac{1}{2} \mathbb{Z}} P_{k}\left(t^{\frac{1}{2}}\right)^{k l} \in U_{P}(\mathfrak{n} \mathfrak{s})\left[\left[t^{\frac{1}{2}}, t^{-\frac{1}{2}}\right]\right] .
$$

Then for $l, n \in 2 \mathbb{Z} \backslash\{0\}$, and $m \in \mathbb{Z}$,

$$
\begin{aligned}
\left(t^{\frac{1}{2}}\right)^{l L_{0}}\left(t^{\frac{1}{2}}\right)^{n L_{0}} & =\left(t^{\frac{1}{2}}\right)^{(l+n) L_{0}}, \\
\left(t^{\frac{1}{2}}\right)^{l L_{0}} L_{m} & =L_{m}\left(t^{\frac{1}{2}}\right)^{-l m}\left(t^{\frac{1}{2}}\right)^{l L_{0}}, \\
\left(t^{\frac{1}{2}}\right)^{l L_{0}} G_{m-\frac{1}{2}} & =G_{m-\frac{1}{2}}\left(t^{\frac{1}{2}}\right)^{-l\left(m-\frac{1}{2}\right)} t^{l L_{0}},
\end{aligned}
$$

and $\left(t^{1 / 2}\right)^{l L_{0}}$ commutes with $d$. From [B4], we have the following proposition.

Proposition 3.1. Let $V$ be a module for $\mathfrak{n s}$ as above. Then there is a unique algebra homomorphism from $U_{P}(\mathfrak{n s})$ to End $V$ such that $L_{j}, G_{j-1 / 2}, d$ and $P_{k}$ are mapped to $L(j), G(j-1 / 2)$, c and $P(k)$, respectively, for $j \in \mathbb{Z}$ and $k \in \frac{1}{2} \mathbb{Z}$.

If $V_{(k)}=0$ for $n$ sufficiently small, then we say that $V$ is a positive energy module for $\mathfrak{n} \mathfrak{s}$ and that the corresponding representation is a positive energy representation of $\mathfrak{n} \mathfrak{s}$. 
3.2. The series $\Gamma$ and $\left\{\Psi_{j}, \Psi_{j-1 / 2}\right\}_{j \in \mathbb{Z}}$. Let $\mathcal{A}=\left\{\mathcal{A}_{j}\right\}_{j \in \mathbb{Z}_{+}}$and $\mathcal{B}=\left\{\mathcal{B}_{j}\right\}_{j \in \mathbb{Z}_{+}}$ be two sequences of even formal variables, let $\mathcal{M}=\left\{\mathcal{M}_{j-1 / 2}\right\}_{j \in \mathbb{Z}_{+}}$and $\mathcal{N}=$ $\left\{\mathcal{N}_{j-1 / 2}\right\}_{j \in \mathbb{Z}_{+}}$be two sequences of odd formal variables, and let $\alpha_{0}^{1 / 2}$ be another even formal variable.

Proposition 3.2. (B⿺辶, BHL) Let $\Psi_{j}$, for $j \in \frac{1}{2} \mathbb{Z}$, be the canonical sequence of formal series given by Theorem [2.3. There exists a unique canonical formal series $\Gamma=\Gamma\left(\alpha_{0}^{1 / 2}, \mathcal{A}, \mathcal{M}, \mathcal{B}, \mathcal{N}\right) \in\left(\mathbb{C}\left[\alpha_{0}^{1 / 2}, \alpha_{0}^{-1 / 2}\right][[\mathcal{A}, \mathcal{B}]][\mathcal{M}, \mathcal{N}]\right)^{0}$ such that

$$
\Gamma=\sum_{j \in \mathbb{Z}_{+}}\left(\left(\frac{j^{3}-j}{12}\right) \alpha_{0}^{-j} \mathcal{A}_{j} \mathcal{B}_{j}+\left(\frac{j^{2}-j}{3}\right) \alpha_{0}^{-j+\frac{1}{2}} \mathcal{N}_{j-\frac{1}{2}} \mathcal{M}_{j-\frac{1}{2}}\right)+\Gamma_{0}
$$

where $\Gamma_{0}$ contains only terms with total degree at least three in the $\mathcal{A}_{j}$ 's, $\mathcal{M}_{j-1 / 2}$ 's, $\mathcal{B}_{j}$ 's, and $\mathcal{N}_{j-1 / 2}$ 's for $j \in \mathbb{Z}_{+}$with each term containing at least one of the $\mathcal{A}_{j}$ 's or $\mathcal{M}_{j-1 / 2}$ 's and at least one of the $\mathcal{B}_{j}$ 's or $\mathcal{N}_{j-1 / 2}$ 's, and such that in the $\mathbb{C}\left[\alpha_{0}^{1 / 2}, \alpha_{0}^{-1 / 2}\right][[\mathcal{A}, \mathcal{B}]][\mathcal{M}, \mathcal{N}]$-envelope of $U_{P}(\mathfrak{n} \mathfrak{s})$, we have

$$
\begin{aligned}
& \text { 3.2) } e^{-\sum_{j \in \mathbb{Z}_{+}}\left(\mathcal{A}_{j} L_{j}+\mathcal{M}_{j-\frac{1}{2}} G_{j-\frac{1}{2}}\right)}\left(\alpha_{0}^{\frac{1}{2}}\right)^{-2 L_{0}} e^{-\sum_{j \in \mathbb{Z}_{+}}\left(\mathcal{B}_{j} L_{-j}+\mathcal{N}_{j-\frac{1}{2}} G_{-j+\frac{1}{2}}\right)} \\
& =e^{\sum_{j \in \mathbb{Z}_{+}}\left(\Psi_{-j} L_{-j}+\Psi_{-j+\frac{1}{2}} G_{-j+\frac{1}{2}}\right)} e^{\sum_{j \in \mathbb{Z}_{+}}\left(\Psi_{j} L_{j}+\Psi_{j-\frac{1}{2}} G_{j-\frac{1}{2}}\right)} e^{2 \Psi_{0} L_{0}}\left(\alpha_{0}^{\frac{1}{2}}\right)^{-2 L_{0}} e^{\Gamma c} .
\end{aligned}
$$

Remark 3.3. In B4 we use the sewing identities on the moduli space on $N=1$ superspheres with tubes to prove Proposition 3.2 above. In BHL, we give a more straightforward and Lie-theoretic proof of Proposition 3.2 by proving a certain bijectivity property for the Campbell-Baker-Hausdorff formula in the theory of Lie algebras. This allows us to prove Proposition 3.2 directly for $U_{P}(\mathfrak{n} \mathfrak{s})$ rather than go through the representation in terms of superderivations and then lift to $U_{P}(\mathfrak{n s})$ as we did in [B2], B4].

Let $V=\bigsqcup_{k \in(1 / 2) \mathbb{Z}} V_{(k)}$ be a module for $\mathfrak{n s}$, and let $L(j), G(j-1 / 2) \in$ End $V$ and $c \in \mathbb{C}$ be the representation images of $L_{j}, G_{j-1 / 2}$ and $d$, respectively, such that $L(0) v=k v$ for $v \in V_{(k)}$.

Corollary 3.4. ([B] $]$ ) In the $\mathbb{C}\left[\alpha_{0}^{1 / 2}, \alpha_{0}^{-1 / 2}\right][[\mathcal{A}, \mathcal{B}]][\mathcal{M}, \mathcal{N}]$-envelope of End $V$, we have

$$
\begin{aligned}
=e^{\sum_{j \in \mathbb{Z}_{+}}\left(\Psi_{-j} L(-j)+\Psi_{-j+\frac{1}{2}} G\left(-j+\frac{1}{2}\right)\right)} \cdot e^{\sum_{j \in \mathbb{Z}_{+}}\left(\Psi_{j} L(j)+\Psi_{j-\frac{1}{2}} G\left(j-\frac{1}{2}\right)\right)} . \\
\cdot e^{2 \Psi_{0} L(0)} \cdot\left(\alpha_{0}^{\frac{1}{2}}\right)^{-2 L(0)} \cdot e^{\Gamma c} .
\end{aligned}
$$

Furthermore, the formal series $\Gamma$ and $\Psi_{j}$, for $j \in \frac{1}{2} \mathbb{Z}$, are actually in

$$
\mathbb{C}[\mathcal{A}][\mathcal{M}][\mathcal{B}][\mathcal{N}]\left[\left[\alpha_{0}^{-\frac{1}{2}}\right]\right] .
$$

Thus letting $(A, M)$ and $(B, N)$ be two sequences in $\bigwedge_{*}^{\infty}$, letting $a_{\square} \in\left(\bigwedge_{*}^{0}\right)^{\times}$, and letting $t^{1 / 2}$ be an even formal variable, we have that $\Psi_{j}\left(t^{-1 / 2} a_{\square}, A, M, B, N\right)$, for $j \in \frac{1}{2} \mathbb{Z}$, and $\Gamma\left(t^{-1 / 2} a_{\square}, A, M, B, N\right)$ are well defined and belong to $\bigwedge_{*}\left[\left[t^{1 / 2}\right]\right]$. If in 
addition, $V$ is a positive-energy module for $\mathfrak{n} \mathfrak{s}$, then the following identity holds in $\left(\text { End }\left(\bigwedge_{*} \otimes_{\mathbb{C}} V\right)\right)^{0}\left[\left[t^{1 / 2}\right]\right]$.

$$
\begin{gathered}
e^{-\sum_{j \in \mathbb{Z}_{+}}\left(A_{j} L(j)+M_{j-\frac{1}{2}} G\left(j-\frac{1}{2}\right)\right)} \cdot\left(t^{-\frac{1}{2}} a_{\square}\right)^{-2 L(0)} . \\
\cdot e^{-\sum_{j \in \mathbb{Z}_{+}}\left(\mathcal{B}_{j} L(-j)+\mathcal{N}_{j-\frac{1}{2}} G\left(-j+\frac{1}{2}\right)\right)} . \\
=e^{\sum_{j \in \mathbb{Z}_{+}}\left(\Psi_{-j}\left(t^{-\frac{1}{2}} a_{\square}, A, M, B, N\right) L(-j)+\Psi_{-j+\frac{1}{2}}\left(t^{-\frac{1}{2}} a_{\square}, A, M, B, N\right) G\left(-j+\frac{1}{2}\right)\right)} . \\
\cdot e^{\sum_{j \in \mathbb{Z}_{+}}\left(\Psi_{j}\left(t^{-\frac{1}{2}} a_{\square}, A, M, B, N\right) L(j)+\Psi_{j-\frac{1}{2}}\left(t^{-\frac{1}{2}} a_{\square}, A, M, B, N\right) G\left(j-\frac{1}{2}\right)\right)} . \\
\cdot e^{2 \Psi_{0}\left(t^{-\frac{1}{2}} a_{\square}, A, M, B, N\right) L(0)} \cdot\left(t^{-\frac{1}{2}} a_{\square}\right)^{-2 L(0)} \cdot e^{\Gamma\left(t^{-\frac{1}{2}} a_{\square}, A, M, B, N\right) c} .
\end{gathered}
$$

Before introducing the definition of $N=1$ supergeometric vertex operator algebra, we present the following conjecture. The Isomorphism Theorem, Theorem 8.1 only holds when this conjecture is true, or in the trivial case in which the representation of the Neveu-Schwarz algebra being considered has zero central charge.

Conjecture 3.5. Let $Q_{1} \in S K(m)$ and $Q_{2} \in S K(n)$ be given by

$$
\begin{array}{r}
Q_{1}=\left(\left(z_{1}, \theta_{1}\right), \ldots,\left(z_{m-1}, \theta_{m-1}\right) ;\left(A^{(0)}, M^{(0)}\right),\left(a^{(1)}, A^{(1)}, M^{(1)}\right),\right. \\
\left.\ldots,\left(a^{(m)}, A^{(m)}, M^{(m)}\right)\right),
\end{array}
$$

and

$Q_{2}=\left(\left(z_{1}^{\prime}, \theta_{1}^{\prime}\right), \ldots,\left(z_{n-1}^{\prime}, \theta_{n-1}^{\prime}\right) ;\left(B^{(0)}, N^{(0)}\right),\left(b^{(1)}, B^{(1)}, N^{(1)}\right), \ldots,\left(b^{(n)}, B^{(n)}, N^{(n)}\right)\right)$.

If the $i$-th tube of $Q_{1}$ can be sewn with the 0 -th tube of $Q_{2}$, then the $t^{1 / 2}$-series

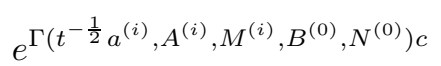

is absolutely convergent at $t^{1 / 2}=1$.

We have already noted the convergence property of the $\Psi_{j}$ 's in Theorem 2.3

3.3. The series $\left\{\Theta_{j}^{(1)}, \Theta_{j-1 / 2}^{(1)}\right\}_{j \in \mathbb{Z}}$ and $\left\{\Theta_{j}^{(2)}, \Theta_{j-1 / 2}^{(2)}\right\}_{j \in \mathbb{Z}}$. Let

$$
\begin{aligned}
H_{\alpha_{0}^{1 / 2}, \mathcal{A}, \mathcal{M}}^{(1)}(x, \varphi) & =\exp \left(-\sum_{j \in \mathbb{Z}_{+}}\left(\mathcal{A}_{j} L_{j}(x, \varphi)+\mathcal{M}_{j-\frac{1}{2}} G_{j-\frac{1}{2}}(x, \varphi)\right)\right) . \\
& =\hat{E}\left(\alpha_{0}^{1 / 2}, \mathcal{A}, \mathcal{M}\right)(x, \varphi) .
\end{aligned}
$$

Let

$$
(\tilde{x}, \tilde{\varphi})=\left(H_{\alpha_{0}^{1 / 2}, \mathcal{A}, \mathcal{M}}^{(1)}\right)^{-1}(x, \varphi) \in\left(\alpha_{0}^{-1} x, \alpha_{0}^{-\frac{1}{2}} \varphi\right)+x \mathbb{C}[x, \varphi]\left[\alpha_{0}^{-\frac{1}{2}}\right][[\mathcal{A}]][\mathcal{M}] .
$$

Let $w$ be another even formal variable and $\rho$ another odd formal variable. Then $s_{(x, \varphi)} \circ H_{\alpha_{0}^{1 / 2}, \mathcal{A}, \mathcal{M}}^{(1)} \circ s_{(\tilde{x}, \tilde{\varphi})}^{-1}\left(\alpha_{0}^{-1} w, \alpha_{0}^{-1 / 2} \rho\right)$ is in

$$
w \mathbb{C}[x, \varphi]\left[\alpha_{0}^{\frac{1}{2}}, \alpha_{0}^{-\frac{1}{2}}\right][[\mathcal{A}]][\mathcal{M}][[w]] \oplus \rho \mathbb{C}[x, \varphi]\left[\alpha_{0}^{\frac{1}{2}}, \alpha_{0}^{-\frac{1}{2}}\right][[\mathcal{A}]][\mathcal{M}][[w]],
$$

is superconformal in $(w, \rho)$, and the even coefficient of the monomial $\rho$ is an element in $\left(1+x \mathbb{C}[x]\left[\alpha_{0}^{1 / 2}, \alpha_{0}^{-1 / 2}\right][[\mathcal{A}]][\mathcal{M}] \oplus \varphi \mathbb{C}[x]\left[\alpha_{0}^{1 / 2}, \alpha_{0}^{-1 / 2}\right][[\mathcal{A}]][\mathcal{M}]\right)$. 
Let $\Theta_{j}^{(1)}=\Theta_{j}^{(1)}\left(\alpha_{0}^{1 / 2}, \mathcal{A}, \mathcal{M},(x, \varphi)\right) \in \mathbb{C}[x, \varphi]\left[\alpha_{0}^{1 / 2}, \alpha_{0}^{-1 / 2}\right][[\mathcal{A}]][\mathcal{M}]$, for $j \in \frac{1}{2} \mathbb{N}$, be defined by

$$
\begin{aligned}
& \left(\operatorname { e x p } \left(\Theta_{0}^{(1)}\left(\alpha_{0}^{\frac{1}{2}}, \mathcal{A}, \mathcal{M},(x, \varphi)\right),\left\{\Theta_{j}^{(1)}\left(\alpha_{0}^{\frac{1}{2}}, \mathcal{A}, \mathcal{M},(x, \varphi)\right),\right.\right.\right. \\
& \left.\left.\Theta_{j-\frac{1}{2}}^{(1)}\left(\alpha_{0}^{\frac{1}{2}}, \mathcal{A}, \mathcal{M},(x, \varphi)\right)\right\}_{j \in \mathbb{Z}_{+}}\right) \\
= & \hat{E}^{-1}\left(s_{(x, \varphi)} \circ H_{\alpha_{0}^{1 / 2}, \mathcal{A}, \mathcal{M}}^{(1)} \circ s_{(\tilde{x}, \tilde{\varphi})}^{-1}\left(\alpha_{0}^{-1} w, \alpha_{0}^{-\frac{1}{2}} \rho\right)\right) .
\end{aligned}
$$

In other words, the $\Theta_{j}^{(1)}$ 's are determined uniquely by

$$
\begin{array}{r}
s_{(x, \varphi)} \circ H_{\alpha_{0}^{1 / 2}, \mathcal{A}, \mathcal{M}}^{(1)} \circ s_{(\tilde{x}, \tilde{\varphi})}^{-1}\left(\alpha_{0}^{-1} w, \alpha_{0}^{-\frac{1}{2}} \rho\right) \\
=\exp \left(\sum_{j \in \mathbb{Z}_{+}}\left(\Theta_{j}^{(1)}\left(w^{j+1} \frac{\partial}{\partial w}+\left(\frac{j+1}{2}\right) \rho w^{j} \frac{\partial}{\partial \rho}\right)+\Theta_{j-\frac{1}{2}}^{(1)} w^{j}\left(\frac{\partial}{\partial \rho}-\rho \frac{\partial}{\partial w}\right)\right)\right) . \\
\exp \left(\Theta_{0}^{(1)}\left(2 w \frac{\partial}{\partial w}+\rho \frac{\partial}{\partial \rho}\right)\right) \cdot(w, \rho) .
\end{array}
$$

This formal power series in $(w, \rho)$ gives the formal local superconformal coordinate at a puncture of the canonical supersphere obtained from the sewing together of two particular canonical superspheres with punctures. Specifically, given a canonical supersphere $Q_{1}$ representing an element in $S K(m)$

$$
\begin{array}{r}
Q_{1}=\left(\left(z_{1}, \theta_{1}\right), \ldots,\left(z_{m-1}, \theta_{m-1}\right) ;\left(A^{(0)}, M^{(0)}\right),\left(a_{\square}^{(1)}, A^{(1)}, M^{(1)}\right), \ldots,\right. \\
\left.\left(a_{\square}^{(m)}, A^{(m)}, M^{(m)}\right)\right)
\end{array}
$$

and a canonical supersphere $Q_{2} \in S K(2)$ with standard local coordinates given by

$$
Q_{2}=\left((z, \theta) ; I(w, \rho), s_{(z, \theta)}(w, \rho),(w, \rho)\right)=((z, \theta) ; \mathbf{0},(1, \mathbf{0}),(1, \mathbf{0}))
$$

and if $\left|z_{B}\right|<r$ where $r$ is the radius of convergence of $\hat{E}\left(a_{\square}^{(m)}, A^{(m)}, M^{(m)}\right)(w, \rho)=$ $H_{\left(a_{\square}^{(m)}, A^{(m)}, M^{(m)}\right)}^{(1)}(w, \rho)$, then we can sew the puncture at infinity of $Q_{2}$ to the $m$-th puncture of $Q_{1}$. In this case, the uniformizing function is given by $F_{\Delta}^{(1)}(w, \rho)=$ $(w, \rho)$ and $F_{\Delta}^{(2)}(w, \rho)=\left(\hat{E}\left(a_{\square}^{(m)}, A^{(m)}, M^{(m)}\right)\right)^{-1}(w, \rho)=\left(H_{\left(a_{\square}^{(m)}, A^{(m)}, M^{(m)}\right)}^{(1)}\right)^{-1}(w, \rho)$.

Thus the resulting canonical supersphere is given by case (1) of Theorem 2.3 and we have

$$
\begin{aligned}
& Q_{1 m} \infty_{0} Q_{2}=\left(\left(z_{1}, \theta_{1}\right), \ldots,\left(z_{m-1}, \theta_{m-1}\right),\left(H_{m}^{(1)}\right)^{-1}(z, \theta) ;\left(A^{(0)}, M^{(0)}\right),\right. \\
& \left(a_{\square}^{(1)}, A^{(1)}, M^{(1)}\right), \ldots,\left(a_{\square}^{(m-1)}, A^{(m-1)}, M^{(m-1)}\right), \\
& \left.\hat{E}^{-1} \circ s_{(z, \theta)} \circ H_{m}^{(1)} \circ s_{\left(H_{m}^{(1)}\right)^{-1}(z, \theta)}^{-1}\left(\left(a_{\square}^{(m)}\right)^{-2} w,\left(a_{\square}^{(m)}\right)^{-1} \rho\right)\right) \\
& =\left(\left(z_{1}, \theta_{1}\right), \ldots,\left(z_{m-1}, \theta_{m-1}\right),\left(H_{m}^{(1)}\right)^{-1}(z, \theta) ;\left(A^{(0)}, M^{(0)}\right)\right. \text {, } \\
& \left(a_{\square}^{(1)}, A^{(1)}, M^{(1)}\right), \ldots,\left(a_{\square}^{(m-1)}, A^{(m-1)}, M^{(m-1)}\right), \\
& \left.\left(\exp \Theta_{0}^{(1)},\left\{\Theta_{j}^{(1)}, \Theta_{j-\frac{1}{2}}^{(1)}\right\}_{j \in \mathbb{Z}_{+}}\right)\right)
\end{aligned}
$$

where $\left(\exp \Theta_{0}^{(1)},\left\{\Theta_{j}^{(1)}, \Theta_{j-\frac{1}{2}}^{(1)}\right\}_{j \in \mathbb{Z}_{+}}\right)$is given formally by (3.5), with $\left(\alpha_{0}^{1 / 2}, \mathcal{A}, \mathcal{M}\right)=$ $\left(a_{\square}^{(m)}, A^{(m)}, M^{(m)}\right)$ and $(x, \varphi)=(z, \theta)$. 
In Section 7 , in order to prove that given an $N=1$ NS-VOSA we can construct an $N=1$ SG-VOSA, we will need the following proposition concerning the series $\Theta_{j}^{(1)}$, for $j \in \frac{1}{2} \mathbb{N}$.

Proposition 3.6. ([B] $)$ In $\left(U(\mathfrak{n s})[x, \varphi]\left[\alpha_{0}^{1 / 2}, \alpha_{0}^{-1 / 2}\right][[\mathcal{A}]][\mathcal{M}]\right)^{0}$, we have

$$
\begin{gathered}
\exp \left(-\sum_{m=-1}^{\infty} \sum_{j \in \mathbb{Z}_{+}}\left(\begin{array}{c}
j+1 \\
m+1
\end{array}\right) \alpha_{0}^{-j} x^{j-m}\left(\left(\mathcal{A}_{j}+2\left(\frac{j-m}{j+1}\right) \alpha_{0}^{\frac{1}{2}} x^{-1} \varphi \mathcal{M}_{j-\frac{1}{2}}\right) L_{m}\right.\right. \\
\left.\left.+x^{-1}\left(\left(\frac{j-m}{j+1}\right) \alpha_{0}^{\frac{1}{2}} \mathcal{M}_{j-\frac{1}{2}}+\varphi \frac{(j-m)}{2} \mathcal{A}_{j}\right) G_{m+\frac{1}{2}}\right)\right) \\
=e^{\left(\left(\tilde{x}-\alpha_{0}^{-1} x\right) L_{-1}+\left(\tilde{\varphi}-\alpha_{0}^{-1 / 2} \varphi\right) G_{-\frac{1}{2}}\right)} \cdot e^{\left(-\sum_{j \in \mathbb{Z}_{+}}\left(\Theta_{j}^{(1)} L_{j}+\Theta_{j-\frac{1}{2}}^{(1)} G_{j-\frac{1}{2}}\right)\right)} \cdot e^{\left(-2 \Theta_{0}^{(1)} L_{0}\right)},
\end{gathered}
$$

for $(\tilde{x}, \tilde{\varphi})=\left(H_{\alpha_{0}^{1 / 2}, \mathcal{A}, \mathcal{M}}^{(1)}\right)^{-1}(x, \varphi)$

Let $V=\coprod_{k \in(1 / 2) \mathbb{Z}} V_{(k)}$ be a module for $\mathfrak{n s}$, and let $L(j), G(j-1 / 2) \in$ End $V$ and $c \in \mathbb{C}$ be the representation images of $L_{j}, G_{j-1 / 2}$ and $d$, respectively, such that $L(0) v=k v$ for $v \in V_{(k)}$.

Corollary 3.7. ([B4]) In ((End $\left.V)[x, \varphi]\left[\alpha_{0}^{1 / 2}, \alpha_{0}^{-1 / 2}\right][[\mathcal{A}]][\mathcal{M}]\right)^{0}$, we have

$$
\begin{aligned}
& \exp \left(-\sum_{m=-1}^{\infty} \sum_{j \in \mathbb{Z}_{+}}\left(\begin{array}{c}
j+1 \\
m+1
\end{array}\right) \alpha_{0}^{-j} x^{j-m}\right. \\
& \left(\left(\mathcal{A}_{j}+2\left(\frac{j-m}{j+1}\right) \alpha_{0}^{\frac{1}{2}} x^{-1} \varphi \mathcal{M}_{j-\frac{1}{2}}\right) L(m)\right. \\
& \left.\left.+x^{-1}\left(\left(\frac{j-m}{j+1}\right) \alpha_{0}^{\frac{1}{2}} \mathcal{M}_{j-\frac{1}{2}}+\varphi \frac{(j-m)}{2} \mathcal{A}_{j}\right) G\left(m+\frac{1}{2}\right)\right)\right) \\
& =e^{\left(\left(\tilde{x}-\alpha_{0}^{-1} x\right) L(-1)+\left(\tilde{\varphi}-\alpha_{0}^{-1 / 2} \varphi\right) G\left(-\frac{1}{2}\right)\right)} \cdot e^{\left(-\sum_{j \in \mathbb{Z}_{+}}\left(\Theta_{j}^{(1)} L(j)+\Theta_{j-\frac{1}{2}}^{(1)} G\left(j-\frac{1}{2}\right)\right)\right)} \cdot \\
& \cdot e^{\left(-2 \Theta_{0}^{(1)} L(0)\right)}
\end{aligned}
$$

for $(\tilde{x}, \tilde{\varphi})=\left(H_{\alpha_{0}^{1 / 2}, \mathcal{A}, \mathcal{M}}^{(1)}\right)^{-1}(x, \varphi)$.

Furthermore, the formal series $\Theta_{j}^{(1)}\left(t^{-1 / 2} \alpha_{0}^{1 / 2}, \mathcal{A}, \mathcal{M},(x, \varphi)\right)$, for $j \in \frac{1}{2} \mathbb{N}$, are in $\mathbb{C}[x, \varphi][\mathcal{A}][\mathcal{M}]\left[\alpha_{0}^{-1 / 2}\right]\left[\left[t^{1 / 2}\right]\right]$. Thus for $a_{\square} \in\left(\bigwedge_{*}^{0}\right)^{\times}$, and $(A, M) \in \bigwedge_{*}^{\infty}$, the series $\Theta_{j}^{(1)}\left(t^{-1 / 2} a_{\square}, A, M,(x, \varphi)\right)$, for $j \in \frac{1}{2} \mathbb{N}$, are well defined and belong to $\bigwedge_{*}[x, \varphi]\left[\left[t^{1 / 2}\right]\right]$.

Let $\left(\tilde{x}\left(t^{1 / 2}\right), \tilde{\varphi}\left(t^{1 / 2}\right)\right)=H_{t^{-1 / 2} a_{\square}, A, M}^{(1)}(x, \varphi)$, and let $V$ be a positive-energy module for $\mathfrak{n s}$. Then in $\left(\left(\text { End }\left(\bigwedge_{*} \otimes_{\mathbb{C}} V\right)\right)\left[\left[t^{\frac{1}{2}}\right]\right]\left[\left[x, x^{-1}\right]\right][\varphi]\right)^{0}$, the following identity holds 
for $\Theta_{j}^{(1)}=\Theta_{j}^{(1)}\left(t^{-1 / 2} a_{\square}, A, M,(x, \varphi)\right)$.

$$
\begin{aligned}
& \exp \left(-\sum_{m=-1}^{\infty} \sum_{j \in \mathbb{Z}_{+}}\left(\begin{array}{c}
j+1 \\
m+1
\end{array}\right) t^{j} a_{\square}^{-2 j} x^{j-m}\right. \\
&\left(\left(A_{j}+2\left(\frac{j-m}{j+1}\right) t^{-\frac{1}{2}} a_{\square} x^{-1} \varphi M_{j-\frac{1}{2}}\right) L(m)\right. \\
&+\left.\left.x^{-1}\left(\left(\frac{j-m}{j+1}\right) t^{-\frac{1}{2}} a_{\square} M_{j-\frac{1}{2}}+\varphi \frac{(j-m)}{2} A_{j}\right) G\left(m+\frac{1}{2}\right)\right)\right) \\
&=\exp \left(\left(\tilde{x}\left(t^{\frac{1}{2}}\right)-\right.\right.\left.\left.t a_{\square}^{-2} x\right) L(-1)+\left(\tilde{\varphi}\left(t^{\frac{1}{2}}\right)-t^{\frac{1}{2}} a_{\square}^{-1} \varphi\right) G\left(-\frac{1}{2}\right)\right) . \\
& \exp \left(-\sum_{j \in \mathbb{Z}_{+}}\left(\Theta_{j}^{(1)} L(j)+\Theta_{j-\frac{1}{2}}^{(1)} G\left(j-\frac{1}{2}\right)\right)\right) \cdot \exp \left(-2 \Theta_{0}^{(1)} L(0)\right) .
\end{aligned}
$$

Let

$$
\begin{aligned}
H^{(2)}(x, \varphi) & =\exp \left(\sum_{j \in \mathbb{Z}_{+}}\left(\mathcal{B}_{j} L_{-j}(x, \varphi)+\mathcal{N}_{j-\frac{1}{2}} G_{-j+\frac{1}{2}}(x, \varphi)\right)\right) \cdot\left(\frac{1}{x}, \frac{i \varphi}{x}\right) \\
& =\tilde{E}(\mathcal{B},-i \mathcal{N})\left(\frac{1}{x}, \frac{i \varphi}{x}\right) .
\end{aligned}
$$

Let

$$
(\tilde{x}, \tilde{\varphi})=\left(H_{\mathcal{B}, \mathcal{N}}^{(2)}\right)^{-1} \circ I(x, \varphi) \in(x, \varphi)+\mathbb{C}\left[x^{-1}, \varphi\right][[\mathcal{B}]][\mathcal{N}] .
$$

Let $w$ be another even formal variable and $\rho$ another odd formal variable. Now write $s_{(x, \varphi)}(w, \rho)=(-x+w-\rho \varphi, \rho-\varphi)$. We will use the convention that we should expand $(-x+w-\rho \varphi)^{j}=(-x+w)^{j}-j \rho \varphi(-x+w)^{j-1}$ in positive powers of the second even variable $w$, for $j \in \mathbb{Z}$. Then

$s_{(x, \varphi)} \circ I^{-1} \circ H_{\mathcal{B}, \mathcal{N}}^{(2)} \circ s_{(\tilde{x}, \tilde{\varphi})}^{-1}(w, \rho) \in w \mathbb{C}\left[x^{-1}, \varphi\right][[\mathcal{B}]][\mathcal{N}][[w]] \oplus \rho \mathbb{C}\left[x^{-1}, \varphi\right][[\mathcal{B}]][\mathcal{N}][[w]]$, is superconformal in $(w, \rho)$, and the even coefficient of the monomial $\rho$ is an element in $\left(1+x^{-1} \mathbb{C}\left[x^{-1}, \varphi\right][[\mathcal{B}]][\mathcal{N}]\right)$.

Let $\Theta_{j}^{(2)}=\Theta_{j}^{(2)}(\mathcal{B}, \mathcal{N},(x, \varphi)) \in \mathbb{C}\left[x^{-1}, \varphi\right][[\mathcal{B}]][\mathcal{N}]$, for $j \in \frac{1}{2} \mathbb{N}$, be defined by

$$
\begin{aligned}
& \left(\exp \left(\Theta_{0}^{(2)}(\mathcal{B}, \mathcal{N},(x, \varphi)),\left\{\Theta_{j}^{(2)}(\mathcal{B}, \mathcal{N},(x, \varphi)), \Theta_{j-\frac{1}{2}}^{(2)}(\mathcal{B}, \mathcal{N},(x, \varphi))\right\}_{j \in \mathbb{Z}_{+}}\right)\right. \\
= & \hat{E}^{-1}\left(s_{(x, \varphi)} \circ I^{-1} \circ H_{\mathcal{B}, \mathcal{N}}^{(2)} \circ s_{(\tilde{x}, \tilde{\varphi})}^{-1}(w, \rho)\right) .
\end{aligned}
$$

In other words, the $\Theta_{j}^{(2)}$ 's are determined uniquely by

$$
\begin{array}{r}
s_{(x, \varphi)} \circ I^{-1} \circ H_{\mathcal{B}, \mathcal{N}}^{(2)} \circ s_{(\tilde{x}, \tilde{\varphi})}^{-1}(w, \rho) \\
=\exp \left(\sum_{j \in \mathbb{Z}_{+}}\left(\Theta_{j}^{(2)}\left(w^{j+1} \frac{\partial}{\partial w}+\left(\frac{j+1}{2}\right) \rho w^{j} \frac{\partial}{\partial \rho}\right)+\Theta_{j-\frac{1}{2}}^{(2)} w^{j}\left(\frac{\partial}{\partial \rho}-\rho \frac{\partial}{\partial w}\right)\right)\right) . \\
\exp \left(\Theta_{0}^{(2)}\left(2 w \frac{\partial}{\partial w}+\rho \frac{\partial}{\partial \rho}\right)\right) \cdot(w, \rho) .
\end{array}
$$


This formal power series in $(w, \rho)$ gives the formal local superconformal coordinate at a puncture of the canonical supersphere obtained from the sewing together of two particular canonical superspheres with punctures. Specifically, given a canonical supersphere $Q_{1}$ representing an element in $S K(2)$ with standard local coordinates given by

$$
Q_{1}=\left((z, \theta) ; I(w, \rho), s_{(z, \theta)}(w, \rho),(w, \rho)\right)=((z, \theta) ; \mathbf{0},(1, \mathbf{0}),(1, \mathbf{0})) \in S K(2)
$$

and a canonical supersphere $Q_{2} \in S K(1)$ given by

$$
Q_{1}=\left(\left(B^{(0)}, N^{(0)}\right),\left(b_{\square}^{(1)}, B^{(1)}, N^{(1)}\right)\right) \in S K(1),
$$

and if $\left|z_{B}\right|>r$ where $r$ is the radius of convergence of $\tilde{E}\left(B^{(0)},-i N^{(0)}\right)(1 / w, i \rho / w)=$ $H_{\left(B^{(0)}, N^{(0)}\right)}^{(2)}(w, \rho)$, then we can sew the puncture at infinity of $Q_{2}$ to the second puncture of $Q_{1}$. In this case, the uniformizing function is given by $F_{\Delta}^{(1)}(w, \rho)=$ $\left(H_{\left(B^{(0)}, N^{(0)}\right)}^{(2)}\right)^{-1} \circ I(w, \rho)$ and $F_{\Delta}^{(2)}(w, \rho)=(w, \rho)$. Thus the resulting canonical supersphere is given by case (1) of Theorem 2.3. and we have

$$
\begin{aligned}
Q_{12} \infty_{0} Q_{2}= & \left(\left(H_{\left(B^{(0)}, N^{(0)}\right)}^{(2)}\right)^{-1} \circ I(z, \theta) ;\left(B^{(0)}, N^{(0)}\right),\right. \\
& \hat{E}^{-1} \circ s_{(z, \theta)} \circ I^{-1} \circ H_{\left(B^{(0)}, N^{(0)}\right)}^{(2)} \circ s_{\left(H_{\left(B^{(0)}, N^{(0)}\right)}^{-1}\right)^{-1}(z, \theta)}^{(2)}(w, \rho), \\
= & \left(\left(H_{\left(B^{(0)}, N^{(0)}\right)}^{(2)}\right)^{-1} \circ I(z, \theta) ;\left(B^{(0)}, N^{(0)}\right),\right. \\
& \left(\exp \Theta_{0}^{(2)},\left\{\Theta_{j}^{(2)}, \Theta_{j-\frac{1}{2}}^{(2)}\right\}_{\left.\left.\left.j \in \mathbb{Z}_{+}\right),\left(b_{\square}^{(1)}, B^{(1)}, N^{(1)}\right)\right)\right)}\right.
\end{aligned}
$$

where $\left(\exp \Theta_{0}^{(2)},\left\{\Theta_{j}^{(2)}, \Theta_{j-\frac{1}{2}}^{(2)}\right\}_{j \in \mathbb{Z}_{+}}\right)$is given formally by (3.8), with $(\mathcal{B}, \mathcal{N})=\left(B^{(0)}, N^{(0)}\right)$ and $(x, \varphi)=(z, \theta)$.

In Section 7 , in order to prove that given an $N=1$ NS-VOSA we can construct an $N=1 \mathrm{SG}$-VOSA, we will need the following proposition concerning the series $\Theta_{j}^{(2)}$, for $j \in \frac{1}{2} \mathbb{N}$.

Proposition 3.8. ([B] ) In $\left(U(\mathfrak{n s})\left[x^{-1}, \varphi\right][[\mathcal{B}]][\mathcal{N}]\right)^{0}$, we have

$$
\begin{gathered}
\exp \left(\sum _ { m = - 1 } ^ { \infty } \sum _ { j \in \mathbb { Z } _ { + } } ( \begin{array} { c } 
{ - j + 1 } \\
{ m + 1 }
\end{array} ) x ^ { - j - m } \left(\left(\mathcal{B}_{j}+2 \varphi \mathcal{N}_{j-\frac{1}{2}}\right) L_{m}\right.\right. \\
\left.\left.+\left(\mathcal{N}_{j-\frac{1}{2}}+\varphi x^{-1} \frac{(-j-m)}{2} \mathcal{B}_{j}\right) G_{m+\frac{1}{2}}\right)\right) \\
=e^{\left((\tilde{x}-x) L_{-1}+(\tilde{\varphi}-\varphi) G_{-\frac{1}{2}}\right)} \cdot e^{\left(-\sum_{j \in \mathbb{Z}_{+}}\left(\Theta_{j}^{(2)} L_{j}+\Theta_{j-\frac{1}{2}}^{(2)} G_{j-\frac{1}{2}}\right)\right)} \cdot e^{\left(-2 \Theta_{0}^{(2)} L_{0}\right)},
\end{gathered}
$$

where $(\tilde{x}, \tilde{\varphi})=\left(H_{\mathcal{B}, \mathcal{N}}^{(2)}\right)^{-1} \circ I(x, \varphi)$.

Let $V=\coprod_{k \in(1 / 2) \mathbb{Z}} V_{(k)}$ be a module for $\mathfrak{n s}$, and let $L(j), G(j-1 / 2) \in$ End $V$ and $c \in \mathbb{C}$ be the representation images of $L_{j}, G_{j-1 / 2}$ and $d$, respectively, such that $L(0) v=k v$ for $v \in V_{(k)}$. 
Corollary 3.9. (B4]) In $\left((\text { End } V)\left[x^{-1}, \varphi\right][[\mathcal{B}]][\mathcal{N}]\right)^{0}$, we have

$$
\begin{array}{r}
\exp \left(\sum _ { m = - 1 } ^ { \infty } \sum _ { j \in \mathbb { Z } _ { + } } ( \begin{array} { c } 
{ - j + 1 } \\
{ m + 1 }
\end{array} ) x ^ { - j - m } \left(\left(\mathcal{B}_{j}+2 \varphi \mathcal{N}_{j-\frac{1}{2}}\right) L(m)\right.\right. \\
\left.\left.+\left(\mathcal{N}_{j-\frac{1}{2}}+\varphi x^{-1} \frac{(-j-m)}{2} \mathcal{B}_{j}\right) G\left(m+\frac{1}{2}\right)\right)\right) \\
\left.=e^{\left((\tilde{x}-x) L(-1)+(\tilde{\varphi}-\varphi) G\left(-\frac{1}{2}\right)\right)} \cdot e^{\left(-\sum_{j \in \mathbb{Z}_{+}}\left(\Theta_{j}^{(2)} L(j)+\Theta_{j-\frac{1}{2}}^{(2)} G\left(j-\frac{1}{2}\right)\right)\right.}\right) \cdot e^{\left(-2 \Theta_{0}^{(2)} L(0)\right)},
\end{array}
$$

for $(\tilde{x}, \tilde{\varphi})=\left(H_{\mathcal{B}, \mathcal{N}}^{(2)}\right)^{-1} \circ I(x, \varphi)$.

Furthermore, the formal series $\Theta_{j}^{(2)}\left(\left\{t^{k} \mathcal{B}_{k}, t^{k-1 / 2} \mathcal{N}_{k-1 / 2}\right\}_{k \in \mathbb{Z}_{+}},(x, \varphi)\right)$, for $j \in$ $\frac{1}{2} \mathbb{N}$, are in $\mathbb{C}\left[x^{-1}, \varphi\right][\mathcal{B}][\mathcal{N}]\left[\left[t^{1 / 2}\right]\right]$. Thus for $(B, N) \in \bigwedge_{*}^{\infty}$, the series

$$
\Theta_{j}^{(2)}\left(\left\{t^{k} B_{k}, t^{k-\frac{1}{2}} N_{k-\frac{1}{2}}\right\}_{k \in \mathbb{Z}_{+}},(x, \varphi)\right)
$$

are well defined and belong to $\bigwedge_{*}\left[x^{-1}, \varphi\right]\left[\left[t^{1 / 2}\right]\right]$.

Let $\left(\tilde{x}\left(t^{1 / 2}\right), \tilde{\varphi}\left(t^{1 / 2}\right)\right)=\left(H_{\left\{t^{j} B_{j}, t^{j-1 / 2} N_{j-1 / 2}\right\}_{j \in \mathbb{Z}_{+}}}^{(2)}\right)^{-1} \circ I(x, \varphi)$, and let $V$ be a positive-energy module for $\mathfrak{n s}$. Then in $\left(\left(\text { End }\left(\bigwedge_{*} \otimes_{\mathbb{C}} V\right)\right)\left[\left[t^{1 / 2}\right]\right]\left[\left[x, x^{-1}\right]\right][\varphi]\right)^{0}$, the following identity holds for $\Theta_{j}^{(2)}=\Theta_{j}^{(2)}\left(\left\{t^{k} B_{k}, t^{k-1 / 2} N_{k-1 / 2}\right\}_{k \in \mathbb{Z}_{+}},(x, \varphi)\right)$.

$$
\begin{aligned}
\exp \left(\sum _ { m = - 1 } ^ { \infty } \sum _ { j \in \mathbb { Z } _ { + } } ( \begin{array} { c } 
{ - j + 1 } \\
{ m + 1 }
\end{array} ) x ^ { - j - m } \left(\left(t^{j} B_{j}+2 \varphi t^{j-\frac{1}{2}} N_{j-\frac{1}{2}}\right) L(m)\right.\right. \\
\left.\left.+\left(t^{j-\frac{1}{2}} N_{j-\frac{1}{2}}+\varphi x^{-1} \frac{(-j-m)}{2} t^{j} B_{j}\right) G\left(m+\frac{1}{2}\right)\right)\right) \\
=\exp \left(\left(\tilde{x}\left(t^{\frac{1}{2}}\right)-x\right) L(-1)+\left(\tilde{\varphi}\left(t^{\frac{1}{2}}\right)-\varphi\right) G\left(-\frac{1}{2}\right)\right) \cdot \\
\exp \left(-\sum_{j \in \mathbb{Z}_{+}}\left(\Theta_{j}^{(2)} L(j)+\Theta_{j-\frac{1}{2}}^{(2)} G\left(j-\frac{1}{2}\right)\right)\right) \cdot \exp \left(-2 \Theta_{0}^{(2)} L(0)\right) .
\end{aligned}
$$

4. $N=1$ SUPERGEOMETRIC VERTEX OPERATOR SUPERALGEBRAS

In this section we introduce the notion of $N=1$ supergeometric vertex operator superalgebra.

4.1. Linear algebra of $\frac{1}{2} \mathbb{Z}$-graded $\bigwedge_{*}$-modules with finite-dimensional homogeneous weight subspaces. Let

$$
V=\coprod_{k \in \frac{1}{2} \mathbb{Z}} V_{(k)}=\coprod_{k \in \frac{1}{2} \mathbb{Z}} V_{(k)}^{0} \oplus \coprod_{k \in \frac{1}{2} \mathbb{Z}} V_{(k)}^{1}=V^{0} \oplus V^{1}
$$

with

$$
\operatorname{dim} V_{(k)}<\infty \text { for } k \in \frac{1}{2} \mathbb{Z}
$$

be a $\frac{1}{2} \mathbb{Z}$-graded (by weight) $\bigwedge_{*}$-module with finite-dimensional homogeneous weight spaces $V_{(k)}$ which is also $\mathbb{Z}_{2}$-graded. Let

$$
V^{\prime}=\coprod_{k \in \frac{1}{2} \mathbb{Z}} V_{(k)}^{*}
$$


be the graded dual space of $V$,

$$
\bar{V}=\prod_{k \in \frac{1}{2} \mathbb{Z}} V_{(k)}=\left(V^{\prime}\right)^{*}
$$

the algebraic completion of $V$, and $\langle\cdot, \cdot\rangle$ the natural pairing between $V^{\prime}$ and $\bar{V}$. For any $n \in \mathbb{N}$, let

$$
\mathcal{S F}_{V}(n)=\operatorname{Hom}_{\Lambda_{*}}\left(V^{\otimes n}, \bar{V}\right) .
$$

For any $m \in \mathbb{Z}_{+}, n \in \mathbb{N}$, and any positive integer $i \leq m$, we define the $t^{1 / 2}$ contraction

$$
\begin{aligned}
i *_{0}: \mathcal{S F}_{V}(m) \times \mathcal{S F}_{V}(n) & \rightarrow \operatorname{Hom}\left(V^{\otimes(m+n-1)}, V\left[\left[t^{\frac{1}{2}}, t^{-\frac{1}{2}}\right]\right]\right) \\
(f, g) & \mapsto\left(f_{i} *_{0} g\right)_{t^{1 / 2}}
\end{aligned}
$$

by

(4.1) $\quad\left(f_{i} *_{0} g\right)_{t^{1 / 2}}\left(v_{1} \otimes \cdots \otimes v_{m+n-1}\right)$

$=\sum_{k \in \frac{1}{2} \mathbb{Z}} f\left(v_{1} \otimes \cdots \otimes v_{i-1} \otimes P(k)\left(g\left(v_{i} \otimes \cdots \otimes v_{i+n-1}\right)\right) \otimes v_{i+n} \otimes \cdots \otimes v_{m+n-1}\right) t^{k}$

for all $v_{1}, \ldots, v_{m+n-1} \in V$, where for any $k \in \frac{1}{2} \mathbb{Z}$, the map $P(k): V \rightarrow V_{(k)}$ is the canonical projection map. This is an obvious extension of the notion of $t$-contraction given in $\mathrm{H} 2]$.

Let

$$
\left\{e_{l^{(k)}}^{(k)} \in V_{(k)} \mid k \in \frac{1}{2} \mathbb{Z}, l^{(k)}=1, \ldots, \operatorname{dim} V_{(k)}\right\}
$$

be a homogeneous basis of $V$, and

$$
\left\{\left(e_{l(k)}^{(k)}\right)^{*} \in V_{(k)}^{*} \mid k \in \frac{1}{2} \mathbb{Z}, l^{(k)}=1, \ldots, \operatorname{dim} V_{(k)}\right\}
$$

the corresponding dual basis of $V^{\prime}$. In terms of these bases, the definition of $t^{1 / 2}$ contraction can be written as

$$
\begin{aligned}
& \left(f_{i} *_{0} g\right)_{t^{1 / 2}}\left(v_{1} \otimes \cdots \otimes v_{m+n-1}\right) \\
& =\sum_{k \in \frac{1}{2} \mathbb{Z}} \sum_{i^{(k)}=1}^{\operatorname{dim} V_{(k)}} f\left(v_{1} \otimes \cdots \otimes v_{i-1} \otimes e_{i^{(k)}}^{(k)} \otimes v_{i+n} \otimes \cdots \otimes v_{m+n-1}\right) . \\
& \cdot\left\langle\left(e_{i^{(k)}}^{(k)}\right)^{*}, g\left(v_{i} \otimes \cdots \otimes v_{i+n-1}\right)\right\rangle t^{k}
\end{aligned}
$$

for any $v_{1}, \ldots, v_{m+n-1} \in V$.

If for arbitrary $v^{\prime} \in V^{\prime}, v_{1}, \ldots, v_{m+n-1} \in V$, the formal Laurent series in $t^{1 / 2}$

$$
\left\langle v^{\prime},\left(f_{i} *_{0} g\right)_{t^{1 / 2}}\left(v_{1} \otimes \cdots \otimes v_{m+n-1}\right)\right\rangle
$$

is absolutely convergent when $t^{1 / 2}=1$, then $\left(f_{i} *_{0} g\right)_{1}$ is well defined as an element of $\mathcal{S F}_{V}(m+n-1)$, and we define the contraction $\left(f_{i} *_{0} g\right)$ in $\mathcal{S F}_{V}(m+n-1)$ of $f$ and $g$ by

$$
\left(f_{i} *_{0} g\right)=\left(f_{i} *_{0} g\right)_{1} .
$$

The following associativity of the $t^{1 / 2}$-contraction follows immediately from the definition. 
Proposition 4.1. Let $l \in \mathbb{Z}_{+}$, and $m, n \in \mathbb{N}$ such that $l+m-1 \in \mathbb{Z}_{+}$, and let $f_{1} \in \mathcal{S F}_{V}(l), f_{2} \in \mathcal{S F}_{V}(m), f_{3} \in \mathcal{S F}_{V}(n)$, and $i, j \in \mathbb{Z}_{+}$such that $1 \leq i \leq l$, and $1 \leq j \leq l+m-1$. Then one of the following holds:

(i) $j<i$ and as formal power series in $t_{1}^{1 / 2}$ and $t_{2}^{1 / 2}$

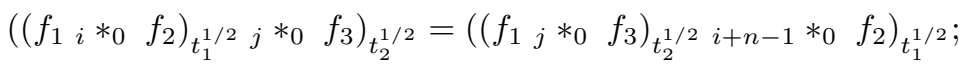

(ii) $j \geq i+m$ and as formal power series in $t_{1}^{1 / 2}$ and $t_{2}^{1 / 2}$

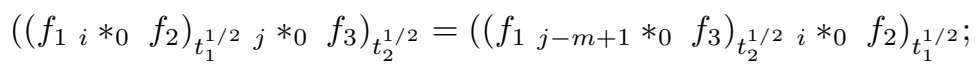

(iii) $i \leq j<i+m$ and as formal power series in $t_{1}^{1 / 2}$ and $t_{2}^{1 / 2}$

$$
\left.\left.\left(\begin{array}{lll}
\left(f_{1 i} *_{0}\right. & f_{2}
\end{array}\right)_{t_{1}^{1 / 2} j} *_{0} f_{3}\right)_{t_{2}^{1 / 2}}=\left(\begin{array}{llll}
f_{1 i} *_{0} & \left(f_{2} j-i+1\right. & *_{0} & f_{3}
\end{array}\right)_{t_{2}^{1 / 2}}\right)_{t_{1}^{1 / 2}} .
$$

Remark 4.2. Note the similarity between Proposition 4.1 and Proposition 2.5 This similarity is due to the fact that both the sewing operation on the moduli space of $N=1$ superspheres with tubes and the $t^{1 / 2}$-contraction define the formal substitution maps for a partial operad structure (cf. [M], [HL1, HL2]) on the moduli space of $N=1$ superspheres with tubes and on the $n$-ary functions from $V$ to $V\left[\left[t^{1 / 2}, t^{-1 / 2}\right]\right]$, respectively. The fact that the associativity of the contraction itself relies on a certain series being doubly absolutely convergent means that the contraction defines only a partial "pseudo-operad" (cf. [HL1, [HL2]) since operad associativity is not automatic.

In addition, an operad has an action of the permutation group which we define below for these $n$-ary functions.

Let $(k l) \in S_{n}$ be the permutation on $n$ letters which switches the $k$-th and $l$-th letters, for $k, l=1, \ldots, n$, with $k<l$. We define the (right) action of the transposition $(k l)$ on $V^{\otimes n}$ by

$$
\begin{aligned}
& \left(v_{1} \otimes \cdots \otimes v_{k-1} \otimes v_{k} \otimes v_{k+1} \otimes \cdots \otimes v_{l-1} \otimes v_{l} \otimes v_{l+1} \otimes \cdots \otimes v_{n}\right) \cdot(k l) \\
& \quad=(-1)^{\eta(k l)}\left(v_{1} \otimes \cdots \otimes v_{k-1} \otimes v_{l} \otimes v_{k+1} \otimes \cdots \otimes v_{l-1} \otimes v_{k} \otimes v_{l+1} \otimes \cdots \otimes v_{n}\right)
\end{aligned}
$$

for $v_{j}$ of homogeneous sign in $V$, where

$$
\eta(k l)=\sum_{j=k+1}^{l-1} \eta\left(v_{j}\right)\left(\eta\left(v_{k}\right)+\eta\left(v_{l}\right)\right)+\eta\left(v_{l}\right) \eta\left(v_{k}\right) .
$$

Let $\sigma \in S_{n}$ be a permutation on $n$ letters. Then $\sigma$ is the product of transpositions $\sigma=\sigma_{1} \cdots \sigma_{m}, \sigma_{i}=\left(k_{i} l_{i}\right)$, for $k_{i}, l_{i}=1, \ldots, n$, with $k_{i}<l_{i}, i=1, \ldots, m$. Thus we have a right action of $S_{n}$ on $V^{\otimes n}$ given by

$$
\begin{aligned}
\left(v_{1} \otimes \cdots \otimes v_{n}\right) \cdot \sigma & =\left(v_{1} \otimes \cdots \otimes v_{n}\right) \cdot \sigma_{1} \cdots \sigma_{m} . \\
& =(-1)^{\eta\left(\sigma_{1}\right)+\cdots+\eta\left(\sigma_{m}\right)} v_{\sigma(1)} \otimes \cdots \otimes v_{\sigma(n)} \\
& =(-1)^{\eta(\sigma)} v_{\sigma(1)} \otimes \cdots \otimes v_{\sigma(n)} .
\end{aligned}
$$

This right action of $S_{n}$ on $n$-tuples in $V$, induces a left action of $S_{n}$ on $\mathcal{S F}_{V}(n)$ given by

$$
(\sigma \cdot f)\left(v_{1} \otimes \cdots \otimes v_{n}\right)=f\left(\left(v_{1} \otimes \cdots \otimes v_{n}\right) \cdot \sigma\right),
$$

for $f \in \mathcal{S F}_{V}(n)$.

Since $V$ is $\mathbb{Z}_{2}$-graded, End $V$ has a natural $\mathbb{Z}_{2}$-grading and a natural Lie superalgebra structure. If $P \in$ End $V$, the corresponding adjoint operator on $V^{\prime}$, if it 
exists, is denoted by $P^{\prime}$. The condition for the existence of $P^{\prime}$ is that the linear functional on $V$ defined by the right-hand side of

$$
\left\langle P^{\prime} v^{\prime}, v\right\rangle=\left\langle v^{\prime}, P v\right\rangle, \text { for } v \in V, v^{\prime} \in V^{\prime}
$$

should lie in $V^{\prime}$. If there exists $n \in \frac{1}{2} \mathbb{Z}$ such that $P$ maps $V_{(k)}$ to $V_{(n+k)}$ for any $k \in \frac{1}{2} \mathbb{Z}$, we say that $P$ has weight $n$. Note that $P$ has weight $n$ if and only if its adjoint $P^{\prime}$ exists and has weight $-n$ as an operator on $V^{\prime}$. In addition, $P$ is even (resp., odd) if and only if its adjoint is even (resp., odd). In the case that $V$ is a module for $\mathfrak{n} \mathfrak{s}$ graded by the eigenvalues of $L(0)$, the adjoint operator $L^{\prime}(-n)$ for $n \in \mathbb{Z}$ corresponding to $L(-n)$ exists and is even with weight $n$, and the adjoint operator $G^{\prime}(-n-1 / 2)$ for $n \in \mathbb{Z}$ corresponding to $G(-n-1 / 2)$ exists and is odd with weight $n+1 / 2$.

\subsection{The notion of $N=1$ supergeometric vertex operator superalgebra.}

Definition 4.3. An $N=1$ supergeometric vertex operator superalgebra over $\bigwedge_{*}$ $\left(N=1 S G\right.$-VOSA) is a $\frac{1}{2} \mathbb{Z}$-graded (by weight) $\bigwedge_{\infty}$-module which is also $\mathbb{Z}_{2}$-graded (by sign)

$$
V=\coprod_{k \in \frac{1}{2} \mathbb{Z}} V_{(k)}=\coprod_{k \in \frac{1}{2} \mathbb{Z}} V_{(k)}^{0} \oplus \coprod_{k \in \frac{1}{2} \mathbb{Z}} V_{(k)}^{1}=V^{0} \oplus V^{1}
$$

such that only $\bigwedge_{*} \subseteq \bigwedge_{\infty}$ acts nontrivially on $V$,

$$
\operatorname{dim} V_{(k)}<\infty \text { for } k \in \frac{1}{2} \mathbb{Z},
$$

and, for any $n \in \mathbb{N}$, a map

$$
\nu_{n}: S K(n) \rightarrow S \mathcal{F}_{V}(n)
$$

satisfying the following axioms:

(1) Positive energy axiom:

$$
V_{(k)}=0 \quad \text { for } k \text { sufficiently small. }
$$

(2) Grading axiom: Let $v^{\prime} \in V^{\prime}, v \in V_{(k)}$, and $a \in\left(\bigwedge_{\infty}^{0}\right)^{\times}$. Then

$$
\left\langle v^{\prime}, \nu_{1}(\mathbf{0},(a, \mathbf{0}))(v)\right\rangle=a^{-2 k}\left\langle v^{\prime}, v\right\rangle .
$$

(3) Supermeromorphicity axiom: For any $n \in \mathbb{Z}_{+}, v^{\prime} \in V^{\prime}$, and $v_{1}, \ldots, v_{n} \in V$, the function

$$
Q \mapsto\left\langle v^{\prime}, \nu_{n}(Q)\left(v_{1} \otimes \cdots \otimes v_{n}\right)\right\rangle
$$

on $S K(n)$ is a canonical supermeromorphic superfunction (in the sense of (2.13)), and if $\left(z_{i}, \theta_{i}\right)$ and $\left(z_{j}, \theta_{j}\right)$, for $i, j \in\{1, \ldots, n\}, i \neq j$, are the $i$-th and $j$-th punctures of $Q \in S K(n)$, respectively, then for any $v_{i}$ and $v_{j}$ in $V$, there exists $N\left(v_{i}, v_{j}\right) \in \mathbb{Z}_{+}$ such that for any $v^{\prime} \in V^{\prime}$ and $v_{l} \in V, l \neq i, j$, the order of the pole $\left(z_{i}, \theta_{i}\right)=\left(z_{j}, \theta_{j}\right)$ of $\left\langle v^{\prime}, \nu_{n}(Q)\left(v_{1} \otimes \cdots \otimes v_{n}\right)\right\rangle$ is less then $N\left(v_{i}, v_{j}\right)$.

(4) Permutation axiom: Let $\sigma \in S_{n}$. Then for any $Q \in S K(n)$

$$
\sigma \cdot\left(\nu_{n}(Q)\right)=\nu_{n}(\sigma \cdot Q) .
$$

(5) Sewing axiom: There exists a unique complex number $c$ (the central charge or rank) such that if $Q_{1} \in S K(m)$ and $Q_{2} \in S K(n)$ are given by

$$
\begin{array}{r}
Q_{1}=\left(\left(z_{1}, \theta_{1}\right), \ldots,\left(z_{m-1}, \theta_{m-1}\right) ;\left(A^{(0)}, M^{(0)}\right),\left(a^{(1)}, A^{(1)}, M^{(1)}\right),\right. \\
\left.\ldots,\left(a^{(m)}, A^{(m)}, M^{(m)}\right)\right),
\end{array}
$$


and

$Q_{2}=\left(\left(z_{1}^{\prime}, \theta_{1}^{\prime}\right), \ldots,\left(z_{n-1}^{\prime}, \theta_{n-1}^{\prime}\right) ;\left(B^{(0)}, N^{(0)}\right),\left(b^{(1)}, B^{(1)}, N^{(1)}\right), \ldots,\left(b^{(n)}, B^{(n)}, N^{(n)}\right)\right)$,

and if the $i$-th tube of $Q_{1}$ can be sewn with the 0-th tube of $Q_{2}$, then for any $v^{\prime} \in V^{\prime}$ and $v_{1}, \ldots, v_{m+n-1} \in V$,

$$
\left\langle v^{\prime},\left(\nu_{m}\left(Q_{1}\right)_{i} *_{0} \quad \nu_{n}\left(Q_{2}\right)\right)_{t^{1 / 2}}\left(v_{1} \otimes \cdots \otimes v_{m+n-1}\right)\right\rangle
$$

is absolutely convergent when $t^{1 / 2}=1$, and

$$
\nu_{m+n-1}\left(Q_{1}{ }_{i} \infty_{0} Q_{2}\right)=\left(\nu_{m}\left(Q_{1}\right)_{i} *_{0} \nu_{n}\left(Q_{2}\right)\right) e^{-\Gamma\left(a^{(i)}, A^{(i)}, M^{(i)}, B^{(0)}, N^{(0)}\right) c} .
$$

Note that in this definition, we assume that Conjecture 3.5 is true.

We denote the $N=1$ SG-VOSA defined above by

$$
\left(V, \nu=\left\{\nu_{n}\right\}_{n \in \mathbb{N}}\right)
$$

or just by $(V, \nu)$.

We can extend this definition to Grassmann algebras over an arbitrary field $\mathbb{F}$ of characteristic zero. But in this case, we have to first introduce the notion of formal superspheres with tubes over the Grassmann algebra over $\mathbb{F}$, and the sewing axiom will become a statement for certain formal series in even and odd variables with coefficients in this Grassmann algebra.

Let $\left(V_{1}, \nu\right)$ and $\left(V_{2}, \mu\right)$ be two $N=1$ SG-VOSAs over $\bigwedge_{*_{1}}$ and $\bigwedge_{*_{2}}$, respectively. A homomorphism of $N=1 S G$-VOSAs is a doubly graded $\wedge_{\infty}$-module map $\gamma$ : $V_{1} \rightarrow V_{2}$ such that if $\bar{\gamma}: \bar{V}_{1} \rightarrow \bar{V}_{2}$ is the unique extension of $\gamma$, then for any $n \in \mathbb{N}$ and any $Q \in S K(n)$

$$
\bar{\gamma} \circ \nu(Q)=\mu(Q) \circ \gamma^{\otimes n} .
$$

Note that an $N=1$ SG-VOSA over some subalgebra of the underlying Grassmann algebra $\bigwedge_{\infty}$ is isomorphic to the same $N=1$ SG-VOSA over a different subalgebra.

\section{5. (Algebraic) $N=1$ Neveu-Schwarz vertex operator Superalgebras}

In this section, we recall the notion of $N=1$ Neveu-Schwarz vertex operator superalgebra over a Grassmann algebra and with odd formal variables $(\mathrm{N}=1 \mathrm{NS}$ VOSA) given in B2] and [B3] and recall some of the consequences of this notion. In addition, in this section we give the formulations of supercommutativity and associativity for an $N=1$ NS-VOSA with odd formal variables.

5.1. Delta functions with odd formal variables. Let $x, x_{0}, x_{1}$ and $x_{2}$ be even formal variables, and let $\varphi, \varphi_{0}, \varphi_{1}$ and $\varphi_{2}$ be odd formal variables. For any formal Laurent series $f(x) \in \bigwedge_{\infty}\left[\left[x, x^{-1}\right]\right]$, we can define

$$
f\left(x+\varphi_{1} \varphi_{2}\right)=f(x)+\varphi_{1} \varphi_{2} f^{\prime}(x) \in \bigwedge_{\infty}\left[\left[x, x^{-1}\right]\right]\left[\varphi_{1}\right]\left[\varphi_{2}\right] .
$$

Recall (cf. [FLM] ) the formal $\delta$-function at $x=1$ given by $\delta(x)=\sum_{n \in \mathbb{Z}} x^{n}$. As developed in B3], we have the following $\delta$-function of expressions involving three even formal variables and two odd formal variables

$$
\begin{aligned}
\delta\left(\frac{x_{1}-x_{2}-\varphi_{1} \varphi_{2}}{x_{0}}\right) & =\sum_{n \in \mathbb{Z}}\left(x_{1}-x_{2}-\varphi_{1} \varphi_{2}\right)^{n} x_{0}^{-n} \\
& =\delta\left(\frac{x_{1}-x_{2}}{x_{0}}\right)-\varphi_{1} \varphi_{2} x_{0}^{-1} \delta^{\prime}\left(\frac{x_{1}-x_{2}}{x_{0}}\right)
\end{aligned}
$$


where $\delta^{\prime}(x)=d / d x \delta(x)=\sum_{n \in \mathbb{Z}} n x^{n-1}$, and we use the conventions that a function of even and odd variables should be expanded about the even variables and any expression in two even variables ( $\operatorname{such}$ as $\left(x_{1}-x_{2}\right)^{n}$, for $n \in \mathbb{Z}$ ) should be expanded in positive powers of the second variable, (in this case $x_{2}$ ).

From [B3], we have the following two $\delta$-function identities

$$
\begin{gathered}
x_{1}^{-1} \delta\left(\frac{x_{2}+x_{0}+\varphi_{1} \varphi_{2}}{x_{1}}\right)=x_{2}^{-1} \delta\left(\frac{x_{1}-x_{0}-\varphi_{1} \varphi_{2}}{x_{2}}\right) \\
x_{0}^{-1} \delta\left(\frac{x_{1}-x_{2}-\varphi_{1} \varphi_{2}}{x_{0}}\right)-x_{0}^{-1} \delta\left(\frac{x_{2}-x_{1}+\varphi_{1} \varphi_{2}}{-x_{0}}\right) \\
=x_{2}^{-1} \delta\left(\frac{x_{1}-x_{0}-\varphi_{1} \varphi_{2}}{x_{2}}\right) .
\end{gathered}
$$

The expressions on both sides of (5.2) and the three terms occurring in (5.3) all correspond to the same formal substitution $x_{0}=x_{1}-x_{2}-\varphi_{1} \varphi_{2}$. Letting $V$ be any $\bigwedge_{\infty}$-module and for

$$
X\left(x_{0}, \varphi_{0}, x_{1}, \varphi_{1}, x_{2}, \varphi_{2}\right) \in(\text { End } V)\left[\left[x_{0}, x_{0}^{-1}, x_{1}, x_{1}^{-1}, x_{2}, x_{2}^{-1}\right]\right]\left[\varphi_{1}, \varphi_{2}\right],
$$

a formal substitution corresponding to $x_{0}=x_{1}-x_{2}-\varphi_{1} \varphi_{2}$ can be made as long as the resulting expression is well defined, e.g., if

$$
X\left(x_{1}, \varphi_{1}, x_{2}, \varphi_{2}\right) \in(\text { End } V)\left[\left[x_{1}, x_{1}^{-1}\right]\right]\left(\left(x_{2}\right)\right)\left[\varphi_{1}, \varphi_{2}\right]
$$

then

$$
\begin{aligned}
\delta\left(\frac{x_{2}+x_{0}+\varphi_{1} \varphi_{2}}{x_{1}}\right) & X\left(x_{1}, \varphi_{1}, x_{2}, \varphi_{2}\right) \\
& =\delta\left(\frac{x_{2}+x_{0}+\varphi_{1} \varphi_{2}}{x_{1}}\right) X\left(x_{2}+x_{0}+\varphi_{1} \varphi_{2}, \varphi_{1}, x_{2}, \varphi_{2}\right),
\end{aligned}
$$

and (5.4) holds for more general $X\left(x_{1}, \varphi_{1}, x_{2}, \varphi_{2}\right)$ as long as the expression $X\left(x_{2}+\right.$ $\left.x_{0}+\varphi_{1} \varphi_{2}, \varphi_{1}, x_{2}, \varphi_{2}\right)$ is well defined. Any such substitution corresponding to $x_{0}=$ $x_{1}-x_{2}-\varphi_{1} \varphi_{2}$ can be thought of as the even part of a superconformal shift of $\left(x_{1}, \varphi_{1}\right)$ by $\left(x_{2}, \varphi_{2}\right)$.

\subsection{The notion of $N=1$ NS-VOSA over $\bigwedge_{*}$ and with odd formal vari-} ables.

Definition 5.1. An $N=1 N S$-VOSA over $\bigwedge_{*}$ and with odd variables is a $\frac{1}{2} \mathbb{Z}$ graded (by weight) $\bigwedge_{\infty}$-module which is also $\mathbb{Z}_{2}$-graded (by sign)

$$
V=\coprod_{k \in \frac{1}{2} \mathbb{Z}} V_{(k)}=\coprod_{k \in \frac{1}{2} \mathbb{Z}} V_{(k)}^{0} \oplus \coprod_{k \in \frac{1}{2} \mathbb{Z}} V_{(k)}^{1}=V^{0} \oplus V^{1}
$$

such that only $\bigwedge_{*} \subseteq \bigwedge_{\infty}$ acts nontrivially,

$$
\begin{gathered}
\operatorname{dim} V_{(k)}<\infty \quad \text { for } \quad k \in \frac{1}{2} \mathbb{Z}, \\
V_{(k)}=0 \quad \text { for } k \text { sufficiently small, }
\end{gathered}
$$


equipped with a linear map $V \otimes V \longrightarrow V\left[\left[x, x^{-1}\right]\right][\varphi]$, or equivalently,

$$
\begin{aligned}
& V \rightarrow(\text { End } V)\left[\left[x, x^{-1}\right]\right][\varphi] \\
& v \mapsto Y(v,(x, \varphi))=\sum_{n \in \mathbb{Z}} v_{n} x^{-n-1}+\varphi \sum_{n \in \mathbb{Z}} v_{n-\frac{1}{2}} x^{-n-1}
\end{aligned}
$$

where $v_{n} \in(\text { End } V)^{\eta(v)}$ and $v_{n-1 / 2} \in(\text { End } V)^{(\eta(v)+1) \bmod 2}$ for $v$ of homogeneous sign in $V, x$ is an even formal variable, and $\varphi$ is an odd formal variable, and where $Y(v,(x, \varphi))$ denotes the vertex operator associated with $v$, and equipped also with two distinguished homogeneous vectors $\mathbf{1} \in V_{(0)}^{0}$ (the vacuum) and $\tau \in V_{(3 / 2)}^{1}$ (the Neveu-Schwarz element). The following conditions are assumed for $u, v \in V$ :

$$
u_{n} v=0 \quad \text { for } n \in \frac{1}{2} \mathbb{Z} \text { sufficiently large; }
$$

$$
Y(\mathbf{1},(x, \varphi))=1 \quad(1 \text { on the right being the identity operator })
$$

the creation property holds:

$$
Y(v,(x, \varphi)) \mathbf{1} \in V[[x]][\varphi] \quad \text { and } \quad \lim _{(x, \varphi) \rightarrow 0} Y(v,(x, \varphi)) \mathbf{1}=v ;
$$

the Jacobi identity holds:

$$
\begin{gathered}
x_{0}^{-1} \delta\left(\frac{x_{1}-x_{2}-\varphi_{1} \varphi_{2}}{x_{0}}\right) Y\left(u,\left(x_{1}, \varphi_{1}\right)\right) Y\left(v,\left(x_{2}, \varphi_{2}\right)\right) \\
-(-1)^{\eta(u) \eta(v)} x_{0}^{-1} \delta\left(\frac{x_{2}-x_{1}+\varphi_{1} \varphi_{2}}{-x_{0}}\right) Y\left(v,\left(x_{2}, \varphi_{2}\right)\right) Y\left(u,\left(x_{1}, \varphi_{1}\right)\right) \\
\quad=x_{2}^{-1} \delta\left(\frac{x_{1}-x_{0}-\varphi_{1} \varphi_{2}}{x_{2}}\right) Y\left(Y\left(u,\left(x_{0}, \varphi_{1}-\varphi_{2}\right)\right) v,\left(x_{2}, \varphi_{2}\right)\right),
\end{gathered}
$$

for $u, v$ of homogeneous sign in $V$; the $N=1$ Neveu-Schwarz algebra relations hold:

$$
\begin{aligned}
{[L(m), L(n)] } & =(m-n) L(m+n)+\frac{1}{12}\left(m^{3}-m\right) \delta_{m+n, 0}(\operatorname{rank} V), \\
{\left[G\left(m+\frac{1}{2}\right), L(n)\right] } & =\left(m-\frac{n-1}{2}\right) G\left(m+n+\frac{1}{2}\right), \\
{\left[G\left(m+\frac{1}{2}\right), G\left(n-\frac{1}{2}\right)\right] } & =2 L(m+n)+\frac{1}{3}\left(m^{2}+m\right) \delta_{m+n, 0}(\operatorname{rank} V),
\end{aligned}
$$

for $m, n \in \mathbb{Z}$, where

$$
G\left(n+\frac{1}{2}\right)=\tau_{n+1}, \quad \text { and } \quad 2 L(n)=\tau_{n+\frac{1}{2}} \quad \text { for } n \in \mathbb{Z},
$$

i.e.,

$$
Y(\tau,(x, \varphi))=\sum_{n \in \mathbb{Z}} G\left(n+\frac{1}{2}\right) x^{-n-\frac{1}{2}-\frac{3}{2}}+2 \varphi \sum_{n \in \mathbb{Z}} L(n) x^{-n-2},
$$

and $\operatorname{rank} V \in \mathbb{C}$;

$$
L(0) v=k v \quad \text { for } \quad k \in \frac{1}{2} \mathbb{Z} \quad \text { and } \quad v \in V_{(k)} ;
$$

and the $G(-1 / 2)$-derivative property holds:

$$
\left(\frac{\partial}{\partial \varphi}+\varphi \frac{\partial}{\partial x}\right) Y(v,(x, \varphi))=Y\left(G\left(-\frac{1}{2}\right) v,(x, \varphi)\right) .
$$


The $N=1$ NS-VOSA just defined is denoted by $(V, Y(\cdot,(x, \varphi)), \mathbf{1}, \tau)$, or for simplicity by $V$.

We have the following consequences of the definition of $N=1$ NS-VOSA with odd formal variables. We have

$$
L(n) \mathbf{1}=G\left(n+\frac{1}{2}\right) \mathbf{1}=0, \quad \text { for } n \geq-1 .
$$

There exists $\omega=(1 / 2) G(-1 / 2) \tau \in V_{(2)}$ such that

$$
Y(\omega,(x, \varphi))=\sum_{n \in \mathbb{Z}} L(n) x^{-n-2}-\frac{\varphi}{2} \sum_{n \in \mathbb{Z}}(n+1) G\left(n-\frac{1}{2}\right) x^{-n-2} .
$$

The supercommutator formula is given by

$$
\begin{aligned}
& {\left[Y\left(u,\left(x_{1}, \varphi_{1}\right)\right), Y\left(v,\left(x_{2}, \varphi_{2}\right)\right)\right]} \\
& \quad=\operatorname{Res}_{x_{0}} x_{2}^{-1} \delta\left(\frac{x_{1}-x_{0}-\varphi_{1} \varphi_{2}}{x_{2}}\right) Y\left(Y\left(u,\left(x_{0}, \varphi_{1}-\varphi_{2}\right)\right) v,\left(x_{2}, \varphi_{2}\right)\right)
\end{aligned}
$$

where $\operatorname{Res}_{x_{0}}$ of a power series in $x_{0}$ is the coefficient of $x_{0}^{-1}$. We have

$$
\begin{aligned}
x_{0}^{2 L(0)} Y(v,(x, \varphi)) x_{0}^{-2 L(0)} & =Y\left(x_{0}^{2 L(0)} v,\left(x_{0}^{2} x, x_{0} \varphi\right)\right) \\
Y\left(e^{x_{0} L(-1)+\varphi_{0} G\left(-\frac{1}{2}\right)} v,(x, \varphi)\right) & =e^{x_{0} \frac{\partial}{\partial x}+\varphi_{0}\left(\frac{\partial}{\partial \varphi}+\varphi \frac{\partial}{\partial x}\right)} Y(v,(x, \varphi)) \\
& =Y\left(v,\left(x_{0}+x+\varphi_{0} \varphi, \varphi_{0}+\varphi\right)\right)
\end{aligned}
$$

and

$$
e^{x_{0} L(-1)+\varphi_{0} G\left(-\frac{1}{2}\right)} Y(v,(x, \varphi)) e^{-x_{0} L(-1)-\varphi_{0} G\left(-\frac{1}{2}\right)}=
$$

$$
\begin{aligned}
& =Y\left(e^{x_{0} L(-1)+\varphi_{0} G\left(-\frac{1}{2}\right)-2 \varphi_{0} \varphi L(-1)} v,(x, \varphi)\right) \\
& =Y\left(v,\left(x+x_{0}+\varphi \varphi_{0}, \varphi+\varphi_{0}\right)\right) .
\end{aligned}
$$

And finally, we have the property of skew-supersymmetry: for $u, v$ of homogeneous sign in $V$

$$
e^{x L(-1)+\varphi G\left(-\frac{1}{2}\right)} Y(v,(-x,-\varphi)) u=(-1)^{\eta(v) \eta(u)} Y(u,(x, \varphi)) v .
$$

Let $\left(V_{1}, Y_{1}(\cdot,(x, \varphi)), \mathbf{1}_{1}, \tau_{1}\right)$ and $\left(V_{2}, Y_{2}(\cdot,(x, \varphi)), \mathbf{1}_{2}, \tau_{2}\right)$ be two $N=1$ NS-VOSAs over $\bigwedge_{*_{1}}$ and $\bigwedge_{*_{2}}$, respectively. A homomorphism of $N=1$ NS-VOSAs is a doubly graded $\bigwedge_{\infty}$-module homomorphism $\gamma: V_{1} \longrightarrow V_{2}$ such that

$$
\gamma\left(Y_{1}(u,(x, \varphi)) v\right)=Y_{2}(\gamma(u),(x, \varphi)) \gamma(v) \quad \text { for } \quad u, v \in V_{1},
$$

$\gamma\left(\mathbf{1}_{1}\right)=\mathbf{1}_{2}$, and $\gamma\left(\tau_{1}\right)=\tau_{2}$.

Of course an $N=1 \mathrm{NS}-\mathrm{VOSA}$ over $\bigwedge_{*}$ is isomorphic to the same $N=1 \mathrm{NS}$ VOSA with coefficients in any other $\Lambda_{*^{\prime}}$.

Remark 5.2. Let $(V, Y(\cdot,(x, \varphi)), \mathbf{1}, \tau)$ be an $N=1$ NS-VOSA. In B3, we also study the notion of $N=1$ NS-VOSA over a Grassmann algebra without odd formal variables and show that $(V, Y(\cdot,(x, 0)), \mathbf{1}, \tau)$ is such an algebra. Conversely, given an $N=1$ NS-VOSA without odd formal variables $(V, Y(\cdot, x), \mathbf{1}, \tau)$, we can define $\tilde{Y}(v,(x, \varphi))=Y(v, x)+\varphi Y(G(-1 / 2) v, x)$, and then $(V, \tilde{Y}(\cdot,(x, \varphi)), \mathbf{1}, \tau)$ is an $N=1$ NS-VOSA with odd formal variables. Using this correspondence, in B3] we prove that the category of $N=1$ NS-VOSAS over $\bigwedge_{*}$ with odd formal variables and with central charge, i.e., rank, $c \in \mathbb{C}$, is isomorphic to the category of $N=1$ NS-VOSAs over $\Lambda_{*}$ without odd formal variables and with central charge 
$c \in \mathbb{C}$. However, in including the odd formal variables the correspondence with the geometry and the role of the operator $G(-1 / 2)$ as in the $G(-1 / 2)$-derivative property is more explicit.

5.3. Expansions of rational superfunctions. Let $\bigwedge_{\infty}\left[x_{1}, x_{2}, \ldots, x_{n}\right]_{S}$ be the ring of rational functions obtained by inverting (localizing with respect to) the set

$$
S=\left\{\sum_{i=1}^{n} a_{i} x_{i} \mid a_{i} \in \bigwedge_{\infty}^{0}, \text { not all }\left(a_{i}\right)_{B}=0\right\} .
$$

Recall the map $\iota_{i_{1} \ldots i_{n}}: \mathbb{F}\left[x_{1}, \ldots, x_{n}\right]_{S} \longrightarrow \mathbb{F}\left[\left[x_{1}, x_{1}^{-1}, \ldots, x_{n}, x_{n}^{-1}\right]\right]$ defined in FLM] where coefficients of elements in $S$ are restricted to the field $\mathbb{F}$. We extend this map to $\bigwedge_{\infty}\left[x_{1}, x_{2}, \ldots, x_{n}\right]_{S}\left[\varphi_{1}, \varphi_{2}, \ldots, \varphi_{n}\right]=\bigwedge_{\infty}\left[x_{1}, \varphi_{1}, x_{2}, \varphi_{2}, \ldots, x_{n}, \varphi_{n}\right]_{S}$ in the obvious way obtaining

$$
\iota_{i_{1} \ldots i_{n}}: \bigwedge_{\infty}\left[x_{1}, \varphi_{1}, \ldots, x_{n}, \varphi_{n}\right]_{S} \longrightarrow \bigwedge_{\infty}\left[\left[x_{1}, x_{1}^{-1}, \ldots, x_{n}, x_{n}^{-1}\right]\right]\left[\varphi_{1}, \ldots, \varphi_{n}\right] .
$$

Let $\bigwedge_{\infty}\left[x_{1}, \varphi_{1}, x_{2}, \varphi_{2}, \ldots, x_{n}, \varphi_{n}\right]_{S^{\prime}}$ be the ring of rational functions obtained by inverting the set

$$
S^{\prime}=\left\{\sum_{\substack{i, j=1 \\ i<j}}^{n}\left(a_{i} x_{i}+a_{i j} \varphi_{i} \varphi_{j}\right) \mid a_{i}, a_{i j} \in \bigwedge_{\infty}^{0}, \text { not all }\left(a_{i}\right)_{B}=0\right\} .
$$

Since we use the convention that a function of even and odd variables should be expanded about the even variables, we have

$$
\frac{1}{\sum_{\substack{i, j=1 \\ i<j}}^{n}\left(a_{i} x_{i}+a_{i j} \varphi_{i} \varphi_{j}\right)}=\frac{1}{\sum_{i=1}^{n} a_{i} x_{i}}-\frac{\sum_{\substack{i, j=1 \\ i<j}}^{n} a_{i j} \varphi_{i} \varphi_{j}}{\left(\sum_{i=1}^{n} a_{i} x_{i}\right)^{2}} .
$$

Thus $\bigwedge_{\infty}\left[x_{1}, \varphi_{1}, x_{2}, \varphi_{2}, \ldots, x_{n}, \varphi_{n}\right]_{S^{\prime}} \subseteq \bigwedge_{\infty}\left[x_{1}, \varphi_{1}, x_{2}, \varphi_{2}, \ldots, x_{n}, \varphi_{n}\right]_{S}$, and $\iota_{i_{1} \ldots i_{n}}$ is well defined on $\bigwedge_{\infty}\left[x_{1}, \varphi_{1}, x_{2}, \varphi_{2}, \ldots, x_{n}, \varphi_{n}\right]_{S^{\prime}}$.

In the case $n=2$, the map $\iota_{12}: \bigwedge_{\infty}\left[x_{1}, \varphi_{1}, x_{2}, \varphi_{2}\right]_{S^{\prime}} \longrightarrow \bigwedge_{\infty}\left[\left[x_{1}, x_{2}\right]\right]\left[\varphi_{1}, \varphi_{2}\right]$ is given by first expanding an element of $\bigwedge_{\infty}\left[x_{1}, \varphi_{1}, x_{2}, \varphi_{2}\right]_{S^{\prime}}$ as a formal series in $\bigwedge_{\infty}\left[x_{1}, \varphi_{1}, x_{2}, \varphi_{2}\right]_{S}$ and then expanding each term as a series in $\bigwedge_{\infty}\left[\left[x_{1}, x_{2}\right]\right]\left[\varphi_{1}, \varphi_{2}\right]$ containing at most finitely many negative powers of $x_{2}$ (using binomial expansions for negative powers of linear polynomials involving both $x_{1}$ and $x_{2}$ ).

5.4. Duality for vertex operator superalgebras. In Section 4.2 we formulated the notion of $N=1 \mathrm{SG}-\mathrm{VOSA}$. In Section [6 we will show that any such object defines an $N=1$ NS-VOSA with odd formal variables. To show that the alleged $N=1$ NS-VOSA satisfies the Jacobi identity, we need the notions of associativity and supercommutativity for an $N=1$ NS-VOSA with odd formal variables as formulated in B2] and B3]. Together, these notions of associativity and (super)commutativity are known as "duality", a term which arose from physics.

Let $(V, Y(\cdot,(x, \varphi)), \mathbf{1}, \tau)$ be an $N=1$ NS-VOSA. As in Section 4.1 let $V^{\prime}$ denote the graded dual space of $V$, let $\bar{V}$ denote the algebraic completion of $V$, and let $\langle\cdot, \cdot\rangle$ denote the natural pairing between $V^{\prime}$ and $\bar{V}$.

Proposition 5.3. (B33] (a) (rationality of products) For $u, v, w \in V$, with $u$, and $v$ of homogeneous sign, and $v^{\prime} \in V^{\prime}$, the formal series

$$
\left\langle v^{\prime}, Y\left(u,\left(x_{1}, \varphi_{1}\right)\right) Y\left(v,\left(x_{2}, \varphi_{2}\right)\right) w\right\rangle,
$$


which involves only finitely many negative powers of $x_{2}$ and only finitely many positive powers of $x_{1}$, lies in the image of the map $\iota_{12}$ :

$$
\left\langle v^{\prime}, Y\left(u,\left(x_{1}, \varphi_{1}\right)\right) Y\left(v,\left(x_{2}, \varphi_{2}\right)\right) w\right\rangle=\iota_{12} f\left(x_{1}, \varphi_{1}, x_{2}, \varphi_{2}\right),
$$

where the (uniquely determined) element $f \in \bigwedge_{\infty}\left[x_{1}, \varphi_{1}, x_{2}, \varphi_{2}\right]_{S^{\prime}}$ is of the form

$$
f\left(x_{1}, \varphi_{1}, x_{2}, \varphi_{2}\right)=\frac{g\left(x_{1}, \varphi_{1}, x_{2}, \varphi_{2}\right)}{x_{1}^{r} x_{2}^{s}\left(x_{1}-x_{2}-\varphi_{1} \varphi_{2}\right)^{t}}
$$

for some $g \in \bigwedge_{\infty}\left[x_{1}, \varphi_{1}, x_{2}, \varphi_{2}\right]$ and $r, s, t \in \mathbb{Z}$.

(b) (supercommutativity) We also have

$$
\left\langle v^{\prime}, Y\left(v,\left(x_{2}, \varphi_{2}\right)\right) Y\left(u,\left(x_{1}, \varphi_{1}\right)\right) w\right\rangle=(-1)^{\eta(u) \eta(v)} \iota_{21} f\left(x_{1}, \varphi_{1}, x_{2}, \varphi_{2}\right),
$$

i.e,

$$
\begin{aligned}
\iota_{12}^{-1}\left\langle v^{\prime}, Y\left(u,\left(x_{1}, \varphi_{1}\right)\right) Y(v,\right. & \left.\left.\left(x_{2}, \varphi_{2}\right)\right) w\right\rangle \\
& =(-1)^{\eta(u) \eta(v)} \iota_{21}^{-1}\left\langle v^{\prime}, Y\left(v,\left(x_{2}, \varphi_{2}\right)\right) Y\left(u,\left(x_{1}, \varphi_{1}\right)\right) w\right\rangle .
\end{aligned}
$$

Proposition 5.4. (B3]) (a) (rationality of iterates) For $u, v, w \in V$, and $v^{\prime} \in V^{\prime}$, the formal series $\left\langle v^{\prime}, Y\left(Y\left(u,\left(x_{0}, \varphi_{1}-\varphi_{2}\right)\right) v,\left(x_{2}, \varphi_{2}\right)\right) w\right\rangle$, which involves only finitely many negative powers of $x_{0}$ and only finitely many positive powers of $x_{2}$, lies in the image of the map $\iota_{20}$ :

$$
\left\langle v^{\prime}, Y\left(Y\left(u,\left(x_{0}, \varphi_{1}-\varphi_{2}\right)\right) v,\left(x_{2}, \varphi_{2}\right)\right) w\right\rangle=\iota_{20} h\left(x_{0}, \varphi_{1}-\varphi_{2}, x_{2}, \varphi_{2}\right),
$$

where the (uniquely determined) element $h \in \bigwedge_{\infty}\left[x_{0}, \varphi_{1}, x_{2}, \varphi_{2}\right]_{S^{\prime}}$ is of the form

$$
h\left(x_{0}, \varphi_{1}-\varphi_{2}, x_{2}, \varphi_{2}\right)=\frac{k\left(x_{0}, \varphi_{1}-\varphi_{2}, x_{2}, \varphi_{2}\right)}{x_{0}^{r} x_{2}^{s}\left(x_{0}+x_{2}-\varphi_{1} \varphi_{2}\right)^{t}}
$$

for some $k \in \bigwedge_{\infty}\left[x_{0}, \varphi_{1}, x_{2}, \varphi_{2}\right]$ and $r, s, t \in \mathbb{Z}$.

(b) The formal series $\left\langle v^{\prime}, Y\left(u,\left(x_{0}+x_{2}+\varphi_{1} \varphi_{2}, \varphi_{1}\right)\right) Y\left(v,\left(x_{2}, \varphi_{2}\right)\right) w\right\rangle$, which involves only finitely many negative powers of $x_{2}$ and only finitely many positive powers of $x_{0}$, lies in the image of $\iota_{02}$, and in fact

$$
\left\langle v^{\prime}, Y\left(u,\left(x_{0}+x_{2}+\varphi_{1} \varphi_{2}, \varphi_{1}\right)\right) Y\left(v,\left(x_{2}, \varphi_{2}\right)\right) w\right\rangle=\iota_{02} h\left(x_{0}, \varphi_{1}-\varphi_{2}, x_{2}, \varphi_{2}\right) .
$$

Proposition 5.5. (B3]) (associativity) We have the following equality of rational functions:

$$
\begin{aligned}
\iota_{12}^{-1}\left\langle v^{\prime}, Y\left(u,\left(x_{1}, \varphi_{1}\right)\right) Y\left(v,\left(x_{2}, \varphi_{2}\right)\right) w\right\rangle \\
\quad=\left.\left(\iota_{20}^{-1}\left\langle v^{\prime}, Y\left(Y\left(u,\left(x_{0}, \varphi_{1}-\varphi_{2}\right)\right) v,\left(x_{2}, \varphi_{2}\right)\right) w\right\rangle\right)\right|_{x_{0}=x_{1}-x_{2}-\varphi_{1} \varphi_{2}}
\end{aligned}
$$

Proposition 5.6. ([B3]) In the presence of the other axioms in the definition of $N=1 N S$-VOSA with odd variables, the Jacobi identity follows from the rationality of products and iterates and supercommutativity and associativity. In particular, the Jacobi identity may be replaced by these properties.

\section{6. $N=1$ NS-VOSAs From $N=1$ SG-VOSAS}

In this section, given an $N=1$ supergeometric VOSA, we construct an (algebraic) $N=1$ NS-VOSA. Since $N=1$ NS- (resp., SG-) VOSAs are isomorphic if taken over different subalgebras of the underlying Grassmann algebra $\Lambda_{\infty}$, we suppress the details of which subalgebras are actually acting non-trivially. 
Let $(V, \nu)$ be an $N=1 \mathrm{SG}-\mathrm{VOSA}$, we define the vacuum $\mathbf{1}_{\nu} \in \bar{V}$ by

$$
\mathbf{1}_{\nu}=\nu_{0}(\mathbf{0})
$$

an element $\tau_{\nu} \in \bar{V}$ by

$$
\tau_{\nu}=-\frac{\partial}{\partial \epsilon} \nu_{0}\left(\mathbf{0}, M\left(\epsilon, \frac{3}{2}\right)\right)
$$

(recall (2.34) $)$; and the vertex operator

$$
Y_{\nu}\left(v_{1},(x, \varphi)\right)=\sum_{n \in \mathbb{Z}}\left(v_{1}\right)_{n} x^{-n-1}+\varphi \sum_{n \in \mathbb{Z}}\left(v_{1}\right)_{n-1 / 2} x^{-n-1}
$$

associated with $v_{1} \in V$ by

$$
\left(v_{1}\right)_{n} v_{2}+\theta\left(v_{1}\right)_{n-\frac{1}{2}} v_{2}=\operatorname{Res}_{z}\left(z^{n} \nu_{2}((z, \theta) ; \mathbf{0},(1, \mathbf{0}),(1, \mathbf{0}))\left(v^{\prime} \otimes v_{1} \otimes v_{2}\right)\right),
$$

where $\operatorname{Res}_{z}$ means taking the residue at the singularity $z=0$, i.e., taking the coefficient of $z^{-1}$.

Proposition 6.1. The elements $\mathbf{1}_{\nu}$ and $\tau_{\nu}$ of $\bar{V}$ are in fact in $V_{(0)}$ and $V_{\left(\frac{3}{2}\right)}$, respectively. If the rank of $(V, \nu)$ is $c$, then $\left(V, Y_{\nu}(\cdot,(x, \varphi)), \mathbf{1}_{\nu}, \tau_{\nu}\right)$ is an $N=1$ $N S$-VOSA with odd formal variables and with rank $c$.

Proof. To see that $\mathbf{1}_{\nu} \in V_{(0)}$, we use the grading and sewing axioms for $(V, \nu)$ to note that for all $a \in\left(\bigwedge_{\infty}^{0}\right)^{\times}$

$$
\begin{aligned}
\left\langle v^{\prime}, \mathbf{1}_{\nu}\right\rangle & =\left\langle v^{\prime}, \nu_{0}(\mathbf{0})\right\rangle \\
& =\left\langle v^{\prime}, \nu_{0}\left((\mathbf{0},(a, \mathbf{0}))_{1} \infty_{0}(\mathbf{0})\right)\right\rangle \\
& =\left\langle v^{\prime},\left(\nu_{1}(\mathbf{0},(a, \mathbf{0}))_{1} *_{0} \nu_{0}(\mathbf{0})\right)\right\rangle e^{-\Gamma(a, \mathbf{0}, \mathbf{0}) c} \\
& =\sum_{k \in \frac{1}{2} \mathbb{Z}}\left\langle v^{\prime}, \nu_{1}(\mathbf{0},(a, \mathbf{0}))\left(P(k)\left(\mathbf{1}_{\nu}\right)\right)\right\rangle \\
& =\sum_{k \in \frac{1}{2} \mathbb{Z}} a^{-2 k}\left\langle v^{\prime}, P(k)\left(\mathbf{1}_{\nu}\right)\right\rangle .
\end{aligned}
$$

Thus $\mathbf{1}_{\nu} \in V_{(0)}$. Similarly for $\tau_{\nu}$, for all $a \in\left(\bigwedge_{\infty}^{0}\right)^{\times}$,

$$
\begin{aligned}
a^{-3}\left\langle v^{\prime}, \tau_{\nu}\right\rangle & =-\left\langle v^{\prime}, \frac{\partial}{\partial \epsilon} \nu_{0}\left(\mathbf{0}, M\left(a^{-3} \epsilon, \frac{3}{2}\right)\right)\right\rangle \\
& =-\left\langle v^{\prime}, \frac{\partial}{\partial \epsilon} \nu_{0}\left((\mathbf{0},(a, \mathbf{0}))_{1} \infty_{0}\left(\mathbf{0}, M\left(\epsilon, \frac{3}{2}\right)\right)\right)\right\rangle \\
& =-\left\langle v^{\prime}, \frac{\partial}{\partial \epsilon}\left(\nu_{1}(\mathbf{0},(a, \mathbf{0}))_{1} *_{0} \nu_{0}\left(\mathbf{0}, M\left(\epsilon, \frac{3}{2}\right)\right)\right)\right\rangle e^{-\Gamma\left(a, \mathbf{0}, \mathbf{0}, \mathbf{0}, M\left(\epsilon, \frac{3}{2}\right)\right) c} \\
& =-\sum_{k \in \frac{1}{2} \mathbb{Z}}\left\langle v^{\prime}, \nu_{1}(\mathbf{0},(a, \mathbf{0}))\left(P(k)\left(\frac{\partial}{\partial \epsilon} \nu_{0}\left(\mathbf{0}, M\left(\epsilon, \frac{3}{2}\right)\right)\right)\right)\right\rangle \\
& =\sum_{k \in \frac{1}{2} \mathbb{Z}} a^{-2 k}\left\langle v^{\prime}, P(k)\left(\tau_{\nu}\right)\right\rangle .
\end{aligned}
$$

Thus $\tau_{\nu} \in V_{\left(\frac{3}{2}\right)}$.

The axioms (5.5), (5.6), and (5.7) are obvious. Since for any $v^{\prime} \in V^{\prime}$ and $v_{1}, v_{2} \in V$, the superfunction $\left\langle v^{\prime}, \nu_{2}((z, \theta) ; \mathbf{0},(1, \mathbf{0}),(1, \mathbf{0}))\left(v_{1} \otimes v_{2}\right)\right\rangle$ is a canonical 
supermeromorphic function in $(z, \theta)$ with poles only at 0 and $\infty$, there exists formal Laurent series $f_{0}(x)$ and $f_{1}(x)$ such that

$$
\left.\left(f_{0}(x)+\varphi f_{1}(x)\right)\right|_{(x, \varphi)=(z, \theta)}=\left\langle v^{\prime}, \nu_{2}((z, \theta) ; \mathbf{0},(1, \mathbf{0}),(1, \mathbf{0}))\left(v_{1} \otimes v_{2}\right)\right\rangle .
$$

From this equality and (6.1), we see that

$$
Y_{\nu}\left(v_{1},(x, \varphi)\right) \in(\text { End } V)\left[\left[x, x^{-1}\right]\right][\varphi] .
$$

By the supermeromorphicity axiom, $\left\langle v^{\prime}, \nu_{2}((z, \theta) ; \mathbf{0},(1, \mathbf{0}),(1, \mathbf{0}))(u \otimes v)\right\rangle$ is supermeromorphic in $(z, \theta)$, and there exists $N(u, v) \in \mathbb{Z}_{+}$such that for any $v^{\prime} \in V^{\prime}$ the order of the pole at 0 is less than $N(u, v)$. Thus by (6.1), we must have $u_{n} v=0$ for $n \geq N(u, v)$, and $n \in \frac{1}{2} \mathbb{Z}$.

For any $(z, \theta) \in\left(\bigwedge_{\infty}^{0}\right)^{\times} \oplus \bigwedge_{\infty}^{1}$, using the sewing and grading axioms,

$$
\begin{aligned}
\left.\left\langle v^{\prime}, Y_{\nu}\left(\mathbf{1}_{\nu},(x, \varphi)\right) v\right\rangle\right|_{(x, \varphi)=(z, \theta)} & =\left.\sum_{k \in \frac{1}{2} \mathbb{Z}}\left\langle v^{\prime}, Y_{\nu}\left(P(k)\left(\mathbf{1}_{\nu}\right),(x, \varphi)\right) v\right\rangle\right|_{(x, \varphi)=(z, \theta)} \\
& =\left\langle v^{\prime},\left(\nu_{2}((z, \theta) ; \mathbf{0},(1, \mathbf{0}),(1, \mathbf{0}))_{1} *_{0} \nu_{0}(\mathbf{0})\right)(v)\right\rangle \\
& =\left\langle v^{\prime}, \nu_{1}\left(((z, \theta) ; \mathbf{0},(1, \mathbf{0}),(1, \mathbf{0}))_{1} \infty_{0}(\mathbf{0})\right)(v)\right\rangle \\
& =\left\langle v^{\prime}, \nu_{1}(\mathbf{0},(1, \mathbf{0}))(v)\right\rangle \\
& =\left\langle v^{\prime}, v\right\rangle .
\end{aligned}
$$

Thus $Y_{\nu}\left(\mathbf{1}_{\nu},(x, \varphi)\right)=1$. Similarly,

$$
\begin{aligned}
\left.\left\langle v^{\prime}, Y_{\nu}(v,(x, \varphi)) \mathbf{1}_{\nu}\right\rangle\right|_{(x, \varphi)=(z, \theta)} & =\left.\sum_{k \in \frac{1}{2} \mathbb{Z}}\left\langle v^{\prime}, Y_{\nu}(v,(x, \varphi)) P(k)\left(\mathbf{1}_{\nu}\right)\right\rangle\right|_{(x, \varphi)=(z, \theta)} \\
& =\left\langle v^{\prime},\left(\nu_{2}((z, \theta) ; \mathbf{0},(1, \mathbf{0}),(1, \mathbf{0}))_{2} *_{0} \nu_{0}(\mathbf{0})\right)(v)\right\rangle \\
& =\left\langle v^{\prime}, \nu_{1}\left(((z, \theta) ; \mathbf{0},(1, \mathbf{0}),(1, \mathbf{0}))_{2} \infty_{0}(\mathbf{0})\right)(v)\right\rangle \\
& =\left\langle v^{\prime}, \nu_{1}\left(\left(A(-z, 1), M\left(-\theta, \frac{1}{2}\right)\right),(1, \mathbf{0})\right)(v)\right\rangle .
\end{aligned}
$$

By the supermeromorphicity axiom, we know that $\left\langle v^{\prime}, \nu_{1}(\cdot)(v)\right\rangle$ is a canonical supermeromorphic function on $S K(2)$. Thus $\left\langle v^{\prime}, \nu_{1}((A(-z, 1), M(-\theta, 1 / 2)),(1, \mathbf{0}))(v)\right\rangle$ is a polynomial in $z$ and $\theta$, and by the grading axiom,

$$
\begin{aligned}
\lim _{(z, \theta) \rightarrow 0}\left\langle v^{\prime}, \nu_{1}\left(\left(A(-z, 1), M\left(-\theta, \frac{1}{2}\right)\right),(1, \mathbf{0})\right)(v)\right\rangle & =\left\langle v^{\prime}, \nu_{1},(\mathbf{0},(1, \mathbf{0}))(v)\right\rangle \\
& =\left\langle v^{\prime}, v\right\rangle
\end{aligned}
$$

Thus $\lim _{(x, \varphi) \rightarrow 0} Y_{\nu}(v,(x, \varphi)) \mathbf{1}_{\nu}=v$ which proves the creation property.

We next prove the axioms involving the $N=1$ Neveu-Schwarz algebra. Using the notation $A M^{(01)}=\left(\left(A^{(0)}, M^{(0)}\right),\left(a^{(1)}, A^{(1)}, M^{(1)}\right)\right) \in S K(1)$, we define operators 
$L(n)$ and $G(n-1 / 2)$, for $n \in \mathbb{Z}$, from $V$ to $\left(V^{\prime}\right)^{*}$ by

$$
\begin{aligned}
-\left.\frac{\partial}{\partial A_{-n}^{(0)}}\left\langle v^{\prime}, \nu_{1}\left(A M^{(01)}\right)(v)\right\rangle\right|_{A M^{(01)}=e} & =\left\langle v^{\prime}, L(n) v\right\rangle, \quad n<0, \\
-\left.\frac{1}{2} \frac{\partial}{\partial a^{(1)}}\left\langle v^{\prime}, \nu_{1}\left(A M^{(01)}\right)(v)\right\rangle\right|_{A M^{(01)}=e} & =\left\langle v^{\prime}, L(0) v\right\rangle, \\
-\left.\frac{\partial}{\partial A_{n}^{(1)}}\left\langle v^{\prime}, \nu_{1}\left(A M^{(01)}\right)(v)\right\rangle\right|_{A M^{(01)}=e} & =\left\langle v^{\prime}, L(n) v\right\rangle, \quad n>0, \\
-\left.\frac{\partial}{\partial M_{-n-\frac{1}{2}}^{(0)}}\left\langle v^{\prime}, \nu_{1}\left(A M^{(01)}\right)(v)\right\rangle\right|_{A M^{(01)}=e} & =\left\langle v^{\prime}, G\left(n+\frac{1}{2}\right) v\right\rangle, \quad n<0, \\
-\left.\frac{\partial}{\partial M_{n-\frac{1}{2}}^{(1)}}\left\langle v^{\prime}, \nu_{1}\left(A M^{(01)}\right)(v)\right\rangle\right|_{A M^{(01)}=e} & =\left\langle v^{\prime}, G\left(n-\frac{1}{2}\right) v\right\rangle, \quad n>0 .
\end{aligned}
$$

From the grading axiom, when $v \in V_{(k)}$, for $k \in \frac{1}{2} \mathbb{Z}$,

$$
\begin{aligned}
\left\langle v^{\prime}, L(0) v\right\rangle & =-\left.\frac{1}{2} \frac{\partial}{\partial a^{(1)}}\left\langle v^{\prime}, \nu_{1}\left(A M^{(01)}\right)(v)\right\rangle\right|_{A M^{(01)}=e} \\
& =-\left.\frac{1}{2} \frac{\partial}{\partial a^{(1)}}\left\langle v^{\prime}, \nu_{1}\left(\mathbf{0},\left(a^{(1)}, \mathbf{0}\right)\right)(v)\right\rangle\right|_{a^{(1)}=1} \\
& =-\left.\frac{1}{2} \frac{\partial}{\partial a^{(1)}}\left(\left(a^{(1)}\right)^{-2 k}\left\langle v^{\prime}, v\right\rangle\right)\right|_{a^{(1)}=1} \\
& =k\left\langle v^{\prime}, v\right\rangle
\end{aligned}
$$

for all $v^{\prime} \in V^{\prime}$. Thus $L(0) v=k v$ for $v \in V_{(k)}$.

By the sewing axiom

$$
\begin{aligned}
& \left\langle v^{\prime}, \nu_{1}\left(A M^{(01)}{ }_{1} \infty_{0} B N^{(01)}\right)(v)\right\rangle \\
& =\left\langle v^{\prime},\left(\nu_{1}\left(A M^{(01)}\right){ }_{1} *_{0} \nu_{1}\left(B N^{(01)}\right)\right)(v)\right\rangle e^{-\Gamma\left(a^{(1)}, A^{(1)}, M^{(1)}, B^{(0)}, N^{(0)}\right) c} .
\end{aligned}
$$

Using this, Proposition 2.12 the bracket operation on $\hat{T}_{e} S K(1)$ (the subspace of the supermeromorphic tangent space at the identity of $S K(1)$ defined in Section [2.6), the definitions for $L(n)$ and $G(n-1 / 2)$ given above, and Proposition 3.2 we obtain

$$
\begin{aligned}
& \sum_{k \in \frac{1}{2} \mathbb{Z}}\left\langle v^{\prime}, L(m) P(k)(L(n) v)\right\rangle-\sum_{k \in \frac{1}{2} \mathbb{Z}}\left\langle v^{\prime}, L(n) P(k)(L(m) v)\right\rangle \\
& =\left\langle v^{\prime},(m-n) L(m+n) v\right\rangle+\left\langle v^{\prime}, \frac{m^{3}-m}{12} c \delta_{m+n, 0} v\right\rangle, \\
& \sum_{k \in \frac{1}{2} \mathbb{Z}}\left\langle v^{\prime}, G\left(m+\frac{1}{2}\right) P(k)(L(n) v)\right\rangle-\sum_{k \in \frac{1}{2} \mathbb{Z}}\left\langle v^{\prime}, L(n) P(k)\left(G\left(m+\frac{1}{2}\right) v\right)\right\rangle \\
& =\left\langle v^{\prime},\left(m-\frac{n-1}{2}\right) G\left(m+n+\frac{1}{2}\right) v\right\rangle,
\end{aligned}
$$




$$
\begin{array}{r}
\sum_{k \in \frac{1}{2} \mathbb{Z}}\left\langle v^{\prime}, G\left(m+\frac{1}{2}\right) P(k)\left(G\left(n-\frac{1}{2}\right) v\right)\right\rangle-\sum_{k \in \frac{1}{2} \mathbb{Z}}\left\langle v^{\prime}, G\left(n-\frac{1}{2}\right) P(k)\left(G\left(m+\frac{1}{2}\right) v\right)\right\rangle \\
=\left\langle v^{\prime}, 2 L(m+n) v\right\rangle+\left\langle v^{\prime}, \frac{m^{2}+m}{3} c \delta_{m+n, 0} v\right\rangle,
\end{array}
$$

for $m, n \in \mathbb{Z}$. Taking $m=0, n \neq 0$ and $v \in V_{(j)}$ in (6.7), we obtain

$$
\left\langle v^{\prime}, L(0) L(n) v\right\rangle=\left\langle v^{\prime},(j-n) L(n) v\right\rangle
$$

Thus $L(0) L(n) v=(j-n) L(n) v$ which implies that $L(n)$ is an operator mapping $V_{(j)}$ to $V_{(j-n)}$. In particular, $L(n)$ is an operator from $V$ to itself. Taking $n=0$, and $v \in V_{(j)}$ in (6.8), we obtain

$$
\left\langle v^{\prime}, L(0) G\left(m+\frac{1}{2}\right) v\right\rangle=\left\langle v^{\prime},\left(j-\left(m+\frac{1}{2}\right)\right) G\left(m+\frac{1}{2}\right) v\right\rangle .
$$

Thus $L(0) G(m+1 / 2) v=(j-(m+1 / 2)) G(m+1 / 2) v$ which implies that $G(m+1 / 2)$ is an operator mapping $V_{(j)}$ to $V_{(j-(m+1 / 2))}$. In particular, $G(m+1 / 2)$ is an operator from $V$ to itself. Thus (6.7), (6.8) and (6.9) are equivalent to the $\mathfrak{n s}$ relations.

By the definition of $\mathcal{G}_{e}(z, \theta)$ (see (2.35), the definition of $\tau_{\nu}$, and the sewing axiom,

$$
\begin{aligned}
\mathcal{G}_{e}(z, \theta)\left(\left\langle v^{\prime}, \nu_{1}(\cdot)(v)\right\rangle\right) & \\
= & \left.\frac{\partial}{\partial \epsilon}\left\langle v^{\prime}, \nu_{1}\left(((z, \theta) ; \mathbf{0},(1, \mathbf{0}),(1, \mathbf{0}))_{1} \infty_{0}\left(\mathbf{0}, M\left(\epsilon, \frac{3}{2}\right)\right)\right)(v)\right\rangle\right|_{\epsilon=0} \\
= & \frac{\partial}{\partial \epsilon}\left\langle v^{\prime},\left(\nu_{2}((z, \theta) ; \mathbf{0},(1, \mathbf{0}),(1, \mathbf{0}))_{1} *_{0} \nu_{0}\left(\mathbf{0}, M\left(\epsilon, \frac{3}{2}\right)\right)\right)(v)\right\rangle \cdot \\
= & \left.e^{-\Gamma\left(1, \mathbf{0}, \mathbf{0}, \mathbf{0}, M\left(\epsilon, \frac{3}{2}\right)\right) c}\right|_{\epsilon=0} \\
= & -(-1)^{\eta\left(v^{\prime}\right)}\left\langle v^{\prime},\left(\nu _ { 2 } ( ( z , \theta ) ; \mathbf { 0 } , ( 1 , \mathbf { 0 } ) , ( 1 , \mathbf { 0 } ) ) \sum _ { 1 } * _ { 0 } \frac { \partial } { \partial \epsilon } \nu _ { 0 } ( \mathbf { 0 } , M ( \epsilon , \frac { 3 } { 2 } ) ) | _ { \epsilon = 0 } \left\langlev^{\prime}, Y_{\nu}(P(v)\rangle\right.\right.\right. \\
& =-\left.(-1)^{\eta\left(v^{\prime}\right)} \sum_{k \in \frac{1}{2} \mathbb{Z} \mathbb{Z}}\left\langle v^{\prime}, Y_{\nu}\left(\tau_{\nu},(x, \varphi)\right) v\right\rangle\right|_{(x, \varphi)=(z, \theta)} .
\end{aligned}
$$


On the other hand, by Proposition 2.11

$$
\begin{aligned}
& \mathcal{G}_{e}(z, \theta)\left(\left\langle v^{\prime}, \nu_{1}(\cdot)(v)\right\rangle\right) \\
& =\left(\left.\sum_{k=0}^{1} \sum_{j \in \mathbb{Z}_{+}} z^{-(2 k-1) j-2+k} \frac{\partial}{\partial M_{j-\frac{1}{2}}^{(k)}}\right|_{e}\right. \\
& \left.\quad+2 \theta\left(\left.z^{-2} \frac{\partial}{\partial a_{0}^{(1)}}\right|_{e}+\left.\sum_{k=0}^{1} \sum_{j \in \mathbb{Z}_{+}} z^{-(2 k-1) j-2} \frac{\partial}{\partial A_{j}^{(k)}}\right|_{e}\right)\right)\left(\left\langle v^{\prime}, \nu_{1}(\cdot)(v)\right\rangle\right) \\
& =\quad-(-1)^{\eta\left(v^{\prime}\right)} \sum_{k=0}^{1} \sum_{j \in \mathbb{Z}_{+}}\left\langle v^{\prime}, z^{-(2 k-1) j-2+k} G\left((2 k-1)\left(j-\frac{1}{2}\right)\right) v\right\rangle \\
& \quad+2 \theta\left\langle v^{\prime}, z^{-2} L(0) v\right\rangle+2 \theta \sum_{k=0}^{1} \sum_{j \in \mathbb{Z}_{+}}\left\langle v^{\prime}, z^{-(2 k-1) j-2} L((2 k-1) j) v\right\rangle \\
& =\quad-\left.(-1)^{\eta\left(v^{\prime}\right)}\left\langle v^{\prime},\left(\sum_{n \in \mathbb{Z}} G\left(n+\frac{1}{2}\right) x^{-n-2}+2 \varphi \sum_{n \in \mathbb{Z}} L(n) x^{-n-2}\right) v\right\rangle\right|_{(x, \varphi)=(z, \theta)} .
\end{aligned}
$$

Thus $Y_{\nu}\left(\tau_{\nu},(x, \varphi)\right)=\sum_{n \in \mathbb{Z}} G(n+1 / 2) x^{-n-1 / 2-3 / 2}+2 \varphi \sum_{n \in \mathbb{Z}} L(n) x^{-n-2}$.

By Proposition [2.10] and the sewing axiom,

$$
\begin{aligned}
&\left.\left\langle v^{\prime}, Y_{\nu}\left(v_{1},(x, \varphi)\right) v_{2}\right\rangle\right|_{(x, \varphi)=\left(z_{0}+z+\theta_{0} \theta, \theta+\theta_{0}\right)} \\
&=\left\langle v^{\prime}, \nu_{2}\left(\left(z_{0}+z+\theta_{0} \theta, \theta+\theta_{0}\right) ; \mathbf{0},(1, \mathbf{0}),(1, \mathbf{0})\right)\left(v_{1} \otimes v_{2}\right)\right\rangle \\
&(6.10)=\left\langle v^{\prime},\left(\nu_{2}((z, \theta) ; \mathbf{0},(1, \mathbf{0}),(1, \mathbf{0})){ }_{1} *_{0} \nu_{1}\left(\left(A\left(-z_{0}, 1\right), M\left(-\theta_{0}, \frac{1}{2}\right)\right),(1, \mathbf{0})\right)\right)\right. \\
&\left.\left(v_{1} \otimes v_{2}\right) \cdot e^{-\Gamma\left(1, \mathbf{0}, \mathbf{0}, A\left(-z_{0}, 1\right), M\left(-\theta_{0}, \frac{1}{2}\right)\right) c}\right\rangle .
\end{aligned}
$$

By taking $\partial / \partial \theta_{0}+\theta_{0} \partial / \partial z_{0}$ of [6.10) and setting $\left(z_{0}, \theta_{0}\right)=0$, we obtain

$$
\begin{gathered}
\left.\left(\left.\left(\frac{\partial}{\partial \theta_{0}}+\theta_{0} \frac{\partial}{\partial z_{0}}\right)\left\langle v^{\prime}, Y_{\nu}\left(v_{1},(x, \varphi)\right) v_{2}\right\rangle\right|_{(x, \varphi)=\left(z_{0}+z+\theta_{0} \theta, \theta+\theta_{0}\right)}\right)\right|_{\left(z_{0}, \theta_{0}\right)=0} \\
=(-1)^{\eta\left(v^{\prime}\right)}\left\langle v^{\prime},\left(\nu_{2}((z, \theta) ; \mathbf{0},(1, \mathbf{0}),(1, \mathbf{0}))_{1} *_{0}\right.\right. \\
\left.\left.\left.\quad\left(\frac{\partial}{\partial \theta_{0}}+\theta_{0} \frac{\partial}{\partial z_{0}}\right) \nu_{1}\left(\left(A\left(-z_{0}, 1\right), M\left(-\theta_{0}, \frac{1}{2}\right)\right),(1, \mathbf{0})\right)\right|_{\left(z_{0}, \theta_{0}\right)=0}\right)\left(v_{1} \otimes v_{2}\right)\right\rangle \\
=(-1)^{\eta\left(v^{\prime}\right)}\left\langle v^{\prime},\left(\nu_{2}((z, \theta) ; \mathbf{0},(1, \mathbf{0}),(1, \mathbf{0}))_{1} *_{0}\right.\right. \\
\left.\left.\left.\frac{\partial}{\partial \theta_{0}} \nu_{1}\left(\left(\mathbf{0}, M\left(-\theta_{0}, \frac{1}{2}\right)\right),(1, \mathbf{0})\right)\right|_{\theta_{0}=0}\right)\left(v_{1} \otimes v_{2}\right)\right\rangle .
\end{gathered}
$$


Since for any $f(x)+\varphi g(x) \in \bigwedge_{\infty}\left[\left[x, x^{-1}\right]\right][\varphi]$,

$$
\begin{aligned}
& \left.\left(\frac{\partial}{\partial \varphi}+\varphi \frac{\partial}{\partial x}\right)(f(x)+\varphi g(x))\right|_{(x, \varphi)=(z, \theta)} \\
& \quad=\theta f^{\prime}(z)+g(z) \\
& =\left.\left(\theta f^{\prime}\left(z_{0}+z\right)+g\left(z_{0}+z\right)\right)\right|_{z_{0}=0} \\
& =\frac{\partial}{\partial \theta_{0}}\left(f\left(z_{0}+z\right)+\theta_{0} \theta f^{\prime}\left(z_{0}+z\right)+\left.\left(\theta+\theta_{0}\right) g\left(z_{0}+z\right)\right|_{\left(z_{0}, \theta_{0}\right)=0}\right. \\
& =\left.\left(\frac{\partial}{\partial \theta_{0}}+\theta_{0} \frac{\partial}{\partial z}\right)\left(f\left(z_{0}+z+\theta_{0} \theta\right)+\left(\theta+\theta_{0}\right) g\left(z_{0}+z+\theta_{0} \theta\right)\right)\right|_{\left(z_{0}, \theta_{0}\right)=0},
\end{aligned}
$$

by the definition of $G(-1 / 2)$ given by (6.5),

$$
\begin{aligned}
\left\langle v^{\prime}\right. & \left.,\left(\frac{\partial}{\partial \varphi}+\varphi \frac{\partial}{\partial x}\right) Y_{\nu}\left(v_{1},(x, \varphi)\right) v_{2}\right\rangle\left.\right|_{(x, \varphi)=(z, \theta)} \\
= & \left.(-1)^{\eta\left(v^{\prime}\right)}\left(\frac{\partial}{\partial \varphi}+\varphi \frac{\partial}{\partial x}\right)\left\langle v^{\prime}, Y_{\nu}\left(v_{1},(x, \varphi)\right) v_{2}\right)\right\rangle\left.\right|_{(x, \varphi)=(z, \theta)} \\
= & \left.\left.(-1)^{\eta\left(v^{\prime}\right)}\left(\frac{\partial}{\partial \theta_{0}}+\theta_{0} \frac{\partial}{\partial z_{0}}\right)\left(\left\langle v^{\prime}, Y_{\nu}\left(v_{1},(x, \varphi)\right) v_{2}\right)\right\rangle\right|_{(x, \varphi)=\left(z_{0}+z+\theta_{0} \theta, \theta+\theta_{0}\right)}\right)\left.\right|_{\left(z_{0}, \theta_{0}\right)=0} \\
= & \left\langle v^{\prime},\left(\left.\nu_{2}((z, \theta) ; \mathbf{0},(1, \mathbf{0}),(1, \mathbf{0}))_{1} *_{0} \frac{\partial}{\partial \theta_{0}} \nu_{1}\left(\left(\mathbf{0}, M\left(-\theta_{0}, \frac{1}{2}\right)\right),(1, \mathbf{0})\right)\right|_{\theta_{0}=0}\right)\left(v_{1} \otimes v_{2}\right)\right\rangle \\
= & \left.\sum_{k \in \frac{1}{2} \mathbb{Z}}\left\langle v^{\prime}, Y_{\nu}\left(P(k)\left(G\left(-\frac{1}{2}\right) v_{1}\right),(x, \varphi)\right) v_{2}\right\rangle\right|_{(x, \varphi)=(z, \theta)} \\
= & \left.\left\langle v^{\prime}, Y_{\nu}\left(G\left(-\frac{1}{2}\right) v_{1},(x, \varphi)\right) v_{2}\right\rangle\right|_{(x, \varphi)=(z, \theta)}
\end{aligned}
$$

or equivalently

$$
\left(\frac{\partial}{\partial \varphi}+\varphi \frac{\partial}{\partial x}\right) Y_{\nu}\left(v_{1},(x, \varphi)\right)=Y_{\nu}\left(G\left(-\frac{1}{2}\right) v_{1},(x, \varphi)\right) .
$$

Finally, we prove the Jacobi identity by proving duality. Using the sewing axiom and Proposition 2.8 and assuming that $\left|\left(z_{1}\right)_{B}\right|>\left|\left(z_{2}\right)_{B}\right|>0$, we have

$$
\begin{aligned}
\left.\left\langle v^{\prime}, Y_{\nu}\left(v_{1},\left(x_{1}, \varphi_{1}\right)\right) Y_{\nu}\left(v_{2},\left(x_{2}, \varphi_{2}\right)\right) v\right\rangle\right|_{\left(x_{i}, \varphi_{i}\right)=\left(z_{i}, \theta_{i}\right)} \\
=\left.\sum_{k \in \frac{1}{2} \mathbb{Z}}\left\langle v^{\prime}, Y_{\nu}\left(v_{1},\left(x_{1}, \varphi_{1}\right)\right) P(k)\left(Y_{\nu}\left(v_{2},\left(x_{2}, \varphi_{2}\right)\right) v\right)\right\rangle\right|_{\left(x_{i}, \varphi_{i}\right)=\left(z_{i}, \theta_{i}\right)} \\
=\left\langle v^{\prime},\left(\nu_{2}\left(\left(z_{1}, \theta_{1}\right) ; \mathbf{0},(1, \mathbf{0}),(1, \mathbf{0})\right)_{2} *_{0} \nu_{2}\left(\left(z_{2}, \theta_{2}\right) ; \mathbf{0},(1, \mathbf{0}),(1, \mathbf{0})\right)\right)\left(v_{1} \otimes v_{2} \otimes v\right)\right\rangle \\
=\left\langle v^{\prime}, \nu_{3}\left(\left(\left(z_{1}, \theta_{1}\right) ; \mathbf{0},(1, \mathbf{0}),(1, \mathbf{0})\right)_{2} \infty_{0}\left(\left(z_{2}, \theta_{2}\right) ; \mathbf{0},(1, \mathbf{0}),(1, \mathbf{0})\right)\right)\left(v_{1} \otimes v_{2} \otimes v\right)\right\rangle \\
=\left\langle v^{\prime}, \nu_{3}\left(\left(z_{1}, \theta_{1}\right),\left(z_{2}, \theta_{2}\right) ; \mathbf{0},(1, \mathbf{0}),(1, \mathbf{0}),(1, \mathbf{0})\right)\left(v_{1} \otimes v_{2} \otimes v\right)\right\rangle .
\end{aligned}
$$

Similarly, when $0<\left|\left(z_{1}\right)_{B}\right|<\left|\left(z_{2}\right)_{B}\right|$,

$$
\begin{aligned}
& \left.\left\langle v^{\prime}, Y_{\nu}\left(v_{2},\left(x_{2}, \varphi_{2}\right)\right) Y_{\nu}\left(v_{1},\left(x_{1}, \varphi_{1}\right)\right) v\right\rangle\right|_{\left(x_{i}, \varphi_{i}\right)=\left(z_{i}, \theta_{i}\right)} \\
& =\left\langle v^{\prime}, \nu_{3}\left(\left(z_{2}, \theta_{2}\right),\left(z_{1}, \theta_{1}\right) ; \mathbf{0},(1, \mathbf{0}),(1, \mathbf{0}),(1, \mathbf{0})\right)\left(v_{2} \otimes v_{1} \otimes v\right)\right\rangle .
\end{aligned}
$$


By the permutation axiom,

$$
\begin{aligned}
& \left\langle v^{\prime}, \nu_{3}\left(\left(z_{1}, \theta_{1}\right),\left(z_{2}, \theta_{2}\right) ; \mathbf{0},(1, \mathbf{0}),(1, \mathbf{0}),(1, \mathbf{0})\right)\left(v_{1} \otimes v_{2} \otimes v\right)\right\rangle \\
& \quad=(-1)^{\eta\left(v_{1}\right) \eta\left(v_{2}\right)}\left\langle v^{\prime}, \nu_{3}\left(\left(z_{2}, \theta_{2}\right),\left(z_{1}, \theta_{1}\right) ; \mathbf{0},(1, \mathbf{0}),(1, \mathbf{0}),(1, \mathbf{0})\right)\left(v_{2} \otimes v_{1} \otimes v\right)\right\rangle
\end{aligned}
$$

for any $\left(z_{1}, \theta_{1}\right),\left(z_{2}, \theta_{2}\right) \in \bigwedge_{\infty}$ satisfying $\left(z_{1}\right)_{B} \neq\left(z_{2}\right)_{B}$, and $\left(z_{1}\right)_{B},\left(z_{2}\right)_{B} \neq 0$. By the supermeromorphicity axiom, both the right-hand side and the left-hand side of the above equality are rational superfunctions of the form

$$
\frac{g\left(\left(z_{1}, \theta_{1}\right),\left(z_{2}, \theta_{2}\right)\right)}{z_{1}^{s_{1}} z_{2}^{s_{2}}\left(z_{1}-z_{2}-\theta_{1} \theta_{2}\right)^{s_{12}}}
$$

where $g\left(\left(z_{1}, \theta_{1}\right),\left(z_{2}, \theta_{2}\right)\right) \in \bigwedge_{\infty}\left[z_{1}, \theta_{1}, z_{2}, \theta_{2}\right]$ and $s_{1}, s_{2}, s_{12} \in \mathbb{N}$. Thus we have proved the rationality and supercommutativity.

To prove associativity, we use the sewing axiom and Proposition 2.8 to see that for $\left|\left(z_{1}\right)_{B}\right|>\left|\left(z_{2}\right)_{B}\right|>\left|\left(z_{1}\right)_{B}-\left(z_{2}\right)_{B}\right|>0$

$$
\begin{aligned}
& \left\langle v^{\prime}, Y_{\nu}\left(Y_{\nu}\left(v_{1},\left(x_{0}, \varphi_{1}-\varphi_{2}\right)\right) v_{2},\left(x_{2}, \varphi_{2}\right)\right) v_{3}\right\rangle \mid \begin{aligned}
\left(x_{0}, \varphi_{1}-\varphi_{2}\right) & =\left(z_{1}-z_{2}-\theta_{1} \theta_{2}, \theta_{1}-\theta_{2}\right), \\
\left(x_{2}, \varphi_{2}\right) & =\left(z_{2}, \theta_{2}\right)
\end{aligned} \\
& =\sum_{k \in \frac{1}{2} \mathbb{Z}}\left\langle v^{\prime}, Y_{\nu}\left(P(k)\left(Y_{\nu}\left(v_{1},\left(x_{0}, \varphi_{1}-\varphi_{2}\right)\right) v_{2}\right),\right.\right. \\
& \left.\left.\left(x_{2}, \varphi_{2}\right)\right) v_{3}\right\rangle \mid \begin{aligned}
\left(x_{0}, \varphi_{1}-\varphi_{2}\right) & =\left(z_{1}-z_{2}-\theta_{1} \theta_{2}, \theta_{1}-\theta_{2}\right), \\
\left(x_{2}, \varphi_{2}\right) & =\left(z_{2}, \theta_{2}\right)
\end{aligned} \\
& =\left\langle v^{\prime},\left(\nu _ { 2 } ( ( z _ { 2 } , \theta _ { 2 } ) ; \mathbf { 0 } , ( 1 , \mathbf { 0 } ) , ( 1 , \mathbf { 0 } ) ) _ { 1 } * _ { 0 } \nu _ { 2 } \left(\left(z_{1}-z_{2}-\theta_{1} \theta_{2}, \theta_{1}-\theta_{2}\right)\right.\right. \text {; }\right. \\
& \left.\mathbf{0},(1, \mathbf{0}),(1, \mathbf{0})))\left(v_{1} \otimes v_{2} \otimes v_{3}\right)\right\rangle \\
& =\left\langle v^{\prime}, \nu_{3}\left(( ( z _ { 2 } , \theta _ { 2 } ) ; \mathbf { 0 } , ( 1 , \mathbf { 0 } ) , ( 1 , \mathbf { 0 } ) ) { } _ { 1 } \infty _ { 0 } \left(\left(z_{1}-z_{2}-\theta_{1} \theta_{2}, \theta_{1}-\theta_{2}\right) ;\right.\right.\right. \\
& \left.\mathbf{0},(1, \mathbf{0}),(1, \mathbf{0})))\left(v_{1} \otimes v_{2} \otimes v_{3}\right)\right\rangle \\
& =\left\langle v^{\prime}, \nu_{3}\left(\left(z_{1}, \theta_{1}\right),\left(z_{2}, \theta_{2}\right) ; \mathbf{0},(1, \mathbf{0}),(1, \mathbf{0}),(1, \mathbf{0})\right)\left(v_{1} \otimes v_{2} \otimes v_{3}\right)\right\rangle \\
& =\left.\left\langle v^{\prime}, Y_{\nu}\left(v_{1},\left(x_{1}, \varphi_{1}\right)\right) Y_{\nu}\left(v_{2},\left(x_{2}, \varphi_{2}\right)\right) v_{3}\right\rangle\right|_{\left(x_{i}, \varphi_{i}\right)=\left(z_{i}, \theta_{i}\right)} .
\end{aligned}
$$

From the rationality, supercommutativity, associativity and Proposition [5.6] we obtain the Jacobi identity.

This finishes the proof that $\left(V, Y_{\nu}, \mathbf{1}_{\nu}, \tau_{\nu}\right)$ is an $N=1$ NS-VOSA.

\section{7. $N=1$ SG-VOSAs From $N=1$ NS-VOSAs}

In this section, given an $N=1$ NS-VOSA, we construct an $N=1$ SG-VOSA, assuming that Conjecture 3.5 is true. Let $(V, Y(\cdot,(x, \varphi)), \mathbf{1}, \tau)$, be an $N=1$ NSVOSA with odd formal variables and $\operatorname{rank} c \in \mathbb{C}$. We define a sequence of maps:

$$
\begin{aligned}
\nu_{n}^{Y}: S K(n) & \rightarrow S F_{V}(n) \\
Q & \mapsto \nu_{n}^{Y}(Q)
\end{aligned}
$$

by

$$
\begin{array}{r}
\left\langle v^{\prime}, \nu_{n}^{Y}\left(\left(z_{1}, \theta_{1}\right), \ldots,\left(z_{n-1}, \theta_{n-1}\right) ;\left(A^{(0)}, M^{(0)}\right),\left(a^{(1)}, A^{(1)}, M^{(1)}\right), \ldots\right.\right. \\
\left.\left.\ldots\left(a^{(n)}, A^{(n)}, M^{(n)}\right)\right)\left(v_{1} \otimes \cdots \otimes v_{n}\right)\right\rangle
\end{array}
$$




$$
\begin{aligned}
& =\iota_{1 \cdots n-1}^{-1}\left\langle e^{-\sum_{j \in \mathbb{Z}_{+}}\left(A_{j}^{(0)} L^{\prime}(j)+M_{j-\frac{1}{2}}^{(0)} G^{\prime}\left(j-\frac{1}{2}\right)\right)} v^{\prime},\right. \\
& Y\left(e^{-\sum_{j \in \mathbb{Z}_{+}}\left(A_{j}^{(1)} L(j)+M_{j-\frac{1}{2}}^{(1)} G\left(j-\frac{1}{2}\right)\right)} \cdot\left(a^{(1)}\right)^{-2 L(0)} \cdot v_{1},\left(x_{1}, \varphi_{1}\right)\right) \cdots \\
& Y\left(e^{-\sum_{j \in \mathbb{Z}_{+}}\left(A_{j}^{(n-1)} L(j)+M_{j-\frac{1}{2}}^{(n-1)} G\left(j-\frac{1}{2}\right)\right)} \cdot\left(a^{(n-1)}\right)^{-2 L(0)} \cdot v_{n-1},\left(x_{n-1}, \varphi_{n-1}\right)\right) . \\
& \left.e^{-\sum_{j \in \mathbb{Z}_{+}}\left(A_{j}^{(n)} L(j)+M_{j-\frac{1}{2}}^{(n)} G\left(j-\frac{1}{2}\right)\right)} \cdot\left(a^{(n)}\right)^{-2 L(0)} \cdot v_{n}\right\rangle\left.\right|_{\left(x_{i}, \varphi_{i}\right)=\left(z_{i}, \theta_{i}\right)}
\end{aligned}
$$

for $n \in \mathbb{Z}_{+}$, and

$$
\left\langle v^{\prime}, \nu_{0}^{Y}\left(A^{(0)}, M^{(0)}\right)\right\rangle=\left\langle e^{-\sum_{j \in \mathbb{Z}_{+}}\left(A_{j}^{(0)} L^{\prime}(j)+M_{j-\frac{1}{2}}^{(0)} G^{\prime}\left(j-\frac{1}{2}\right)\right)} v^{\prime}, \mathbf{1}\right\rangle
$$

where $\iota_{1 \cdots n-1}^{-1}$ is defined in Section 5.3

To prove that $\left(V, \nu^{Y}\right)$ is an $N=1 \mathrm{SG}-\mathrm{VOSA}$, and in particular, that it satisfies the sewing axiom, we will need the following proposition.

Proposition 7.1. Let $l \in \mathbb{Z}_{+}$, and $m, n \in \mathbb{N}$ such that $l+m-1 \in \mathbb{Z}_{+}$, and let $Q_{1} \in S K(l), Q_{2} \in S K(m), Q_{3} \in S K(n)$, and $i, j \in \mathbb{Z}_{+}$such that $1 \leq i \leq l$, and $1 \leq j \leq l+m-1$. If the sewings $\left(Q_{1}{ }_{i} \infty_{0} Q_{2}\right){ }_{j} \infty_{0} Q_{3}$ exist, then for any $v^{\prime} \in V^{\prime}$, $v_{1}, \ldots, v_{l+m+n-2} \in V$, the series

$$
\left\langle v^{\prime},\left(\left(\nu_{l}^{Y}\left(Q_{1}\right)_{i} *_{0} \nu_{m}^{Y}\left(Q_{2}\right)\right)_{t_{1}^{1 / 2} j *_{0}} \nu_{n}^{Y}\left(Q_{3}\right)\right)_{t_{2}^{1 / 2}}\left(v_{1} \otimes \cdots \otimes v_{l+m+n-2}\right)\right\rangle
$$

is doubly absolutely convergent at $t_{1}^{1 / 2}=t_{2}^{1 / 2}=1$.

Similarly, for $1 \leq i \leq l, 1 \leq j \leq m$, if the sewings $Q_{1}{ }_{i} \infty_{0}\left(Q_{2}{ }_{j} \infty_{0} Q_{3}\right)$ exist, then for any $v^{\prime} \in V^{\prime}, v_{1}, \ldots, v_{l+m+n-2} \in V$, the series

$$
\left\langle v^{\prime},\left(\nu_{l}^{Y}\left(Q_{1}\right)_{i} *_{0}\left(\nu_{m}^{Y}\left(Q_{2}\right)_{j} *_{0} \nu_{n}^{Y}\left(Q_{3}\right)\right)_{t_{1}^{1 / 2}}\right)_{t_{2}^{1 / 2}}\left(v_{1} \otimes \cdots \otimes v_{l+m+n-2}\right)\right\rangle
$$

is doubly absolutely convergent at $t_{1}^{1 / 2}=t_{2}^{1 / 2}=1$.

Proof. We prove the first statement. The second statement is proved similarly. Let $t_{1}^{1 / 2}, t_{2}^{1 / 2} \in \mathbb{C}^{\times}$. Let $Q_{2}\left(t_{1}^{1 / 2}\right)$ and $Q_{3}\left(t_{2}^{1 / 2}\right)$ be the elements of $S K(m)$ and $S K(n)$, respectively, obtained from $Q_{2}$ and $Q_{3}$, respectively, by multiplying the local coordinate maps at the $i$-th puncture of $Q_{2}$ and the $j$-th puncture of $Q_{3}$ by $t_{1}$ and $t_{2}$, respectively, in the even coordinate, and by $t_{1}^{1 / 2}$ and $t_{2}^{1 / 2}$, respectively, in the odd coordinate. Then there exist two neighborhoods $\Delta_{1}$ and $\Delta_{2}$ of $1 \in \mathbb{C}$ such that for any $\left(t_{1}^{1 / 2}, t_{2}^{1 / 2}\right) \in \Delta_{1} \times \Delta_{2}$, the sewings $\left(Q_{1}{ }_{i} \infty_{0} Q_{2}\left(t_{1}^{1 / 2}\right)\right){ }_{j} \infty_{0} Q_{3}\left(t_{2}^{1 / 2}\right)$ exist.

This gives a two-parameter family of superspheres

$$
\left\{\left(Q_{1}{ }_{i} \infty_{0} Q_{2}\left(t_{1}^{\frac{1}{2}}\right)\right){ }_{j} \infty_{0} Q_{3}\left(t_{2}^{\frac{1}{2}}\right) \mid\left(t_{1}^{\frac{1}{2}}, t_{2}^{\frac{1}{2}}\right) \in \Delta_{1} \times \Delta_{2}\right\} .
$$

Then as in the proof of Proposition 4.19 in [B4], we can take the fiber bundle structure of these superspheres and define global sections to obtain a complex analytic family of compact complex manifolds. Then using the definition of $\nu^{Y}$ and the contraction $*$, the proof that (7.2) is doubly absolutely convergent at $t_{1}^{1 / 2}=t_{2}^{1 / 2}=1$, is completely analogous to the proof of the convergence of the series $\Psi_{j}\left(t^{1 / 2}\right)$ in Proposition 4.19 in [B3], and similarly for (7.3).

Proposition 7.2. The pair $\left(V, \nu^{Y}\right)$ is an $N=1 S G-V O S A$. 
Proof. We must check that $\left(V, \nu^{Y}\right)$ satisfies the five axioms in the definition of an $N=1$ SG-VOSA.

(1) Positive energy axiom: This is obvious.

(2) Grading axiom: For any $v^{\prime} \in V^{\prime}, v \in V_{(k)}$, for $k \in \frac{1}{2} \mathbb{Z}$, and $a \in\left(\bigwedge_{\infty}^{0}\right)^{\times}$,

$$
\left\langle v^{\prime}, \nu_{1}^{Y}(\mathbf{0},(a, \mathbf{0}))(v)\right\rangle=\left\langle v^{\prime}, a^{-2 L(0)} v\right\rangle=a^{-2 k}\left\langle v^{\prime}, v\right\rangle .
$$

(3) Supermeromorphicity axiom: The fact that for any $n \in \mathbb{Z}_{+}, v^{\prime} \in V^{\prime}$, $v_{1}, \ldots, v_{n} \in V$, and $Q \in S K(n)$, the function $Q \mapsto\left\langle v^{\prime}, \nu_{n}^{Y}(Q)\left(v_{1} \otimes \cdots \otimes v_{n}\right)\right\rangle$ is a canonical supermeromorphic function follows from the rationality of $(V, Y, \mathbf{1}, \tau)$ and the fact that $V$ is a positive energy module for $\mathfrak{n s}$. To prove the rest of the supermeromorphicity axiom, without loss of generality, we only discuss those $Q$ of the form $\left(\left(z_{1}, \theta_{1}\right), \ldots,\left(z_{n-1}, \theta_{n-1}\right) ; \mathbf{0},(1, \mathbf{0}), \ldots,(1, \mathbf{0})\right)$.

Take $N\left(v_{i}, v_{j}\right) \in \frac{1}{2} \mathbb{Z}_{+}$such that $\left(v_{i}\right)_{k} v_{j}=0$ for any $k \geq N\left(v_{i}, v_{j}\right)$. If $i<j$, then by supercommutativity and associativity, we have

$$
\begin{aligned}
& \left\langle v^{\prime}, \nu_{1}^{Y}(Q)\left(v_{1} \otimes \cdots \otimes v_{n}\right)\right\rangle \\
& \left.=\iota_{1 \cdots n-1}^{-1}\left(\left\langle v^{\prime}, Y\left(v_{1},\left(x_{1}, \varphi_{1}\right)\right) \cdots Y\left(v_{n-1}, x_{n-1}, \varphi_{n-1}\right)\right) v_{n}\right\rangle\right)\left.\right|_{\left(x_{l}, \varphi_{l}\right)=\left(z_{l}, \varphi_{l}\right)} \\
& =(-1)^{\sum_{m=1}^{j-i-1} \eta\left(v_{j-m}\right) \eta\left(v_{j}\right)} \iota_{1 \cdots(i-1) i j(i+1) \cdots(j-1)(j+1) \cdots n-1}^{-1}\left(\left\langlev^{\prime}, Y\left(v_{1},\left(x_{1}, \varphi_{1}\right)\right) \cdots\right.\right. \\
& Y\left(v_{i-1},\left(x_{i-1}, \varphi_{i-1}\right)\right) Y\left(v_{i},\left(x_{i}, \varphi_{i}\right)\right) Y\left(v_{j},\left(x_{j}, \varphi_{j}\right)\right) Y\left(v_{i+1},\left(x_{i+1}, \varphi_{i+1}\right)\right) \cdots \\
& Y\left(v_{j-1},\left(x_{j-1}, \varphi_{j-1}\right)\right) Y\left(v_{j+1},\left(x_{j+1}, \varphi_{j+1}\right)\right) \cdots \\
& \left.\left.Y\left(v_{n-1},\left(x_{n-1}, \varphi_{n-1}\right)\right) v_{n}\right\rangle\right)\left.\right|_{\left(x_{l}, \varphi_{l}\right)=\left(z_{l}, \theta_{l}\right)} \\
& =(-1)^{\sum_{m=1}^{j-i-1} \eta\left(v_{j-m}\right) \eta\left(v_{j}\right)} \iota_{1 \cdots(i-1) j(i+1) \cdots(j-1)(j+1) \cdots(n-1) 0}^{-1}\left(\left\langlev^{\prime}, Y\left(v_{1},\left(x_{1}, \varphi_{1}\right)\right) \cdots\right.\right. \\
& Y\left(v_{i-1},\left(x_{i-1}, \varphi_{i-1}\right)\right) Y\left(Y\left(v_{i},\left(x_{0}, \varphi_{i}-\varphi_{j}\right)\right) v_{j},\left(x_{j}, \varphi_{j}\right)\right) Y\left(v_{i+1},\left(x_{i+1}, \varphi_{i+1}\right)\right) \\
& \cdots Y\left(v_{j-1},\left(x_{j-1}, \varphi_{j-1}\right)\right) Y\left(v_{j+1},\left(x_{j+1}, \varphi_{j+1}\right)\right) \cdots \\
& \left.\left.Y\left(v_{n-1},\left(x_{n-1}, \varphi_{n-1}\right)\right) v_{n}\right\rangle\right) \mid \begin{array}{c}
\left(x_{l}, \varphi_{l}\right)=\left(z_{l}, \varphi_{l}\right), \text { for } l \neq i, \\
x_{0}=z_{i}-z_{j}-\varphi_{i} \varphi_{j} .
\end{array}
\end{aligned}
$$

By the definition of $N\left(v_{i}, v_{j}\right)$, we see that the order of the pole $\left(z_{i}, \theta_{i}\right)=\left(z_{j}, \theta_{j}\right)$ of $\left\langle v^{\prime}, \nu_{n}^{Y}(Q)\left(v_{1} \otimes \cdots \otimes v_{n}\right)\right\rangle$ is less than $N\left(v_{i}, v_{j}\right)$. The result for $i>j$ is proved similarly.

(4) Permutation axiom: If $\sigma \in S_{n-1} \subset S_{n}$ is a permutation on the first $n-1$ letters, then from supercommutativity, we have

$$
\begin{aligned}
\left\langle v^{\prime}, \nu_{n}^{Y}(\sigma \cdot Q)\left(v_{1} \otimes \cdots \otimes v_{n}\right)\right\rangle & \\
= & \left\langle v^{\prime}, \nu_{n}^{Y}\left(\sigma \cdot \left(\left(z_{1}, \theta_{1}\right), \ldots,\left(z_{n-1}, \theta_{n-1}\right) ;\left(A^{(0)}, M^{(0)}\right),\left(a^{(1)}, A^{(1)}, M^{(1)}\right), \ldots,\right.\right.\right. \\
& \left.\left.\left.\left(a^{(n)}, A^{(n)}, M^{(n)}\right)\right)\right)\left(v_{1} \otimes \cdots \otimes v_{n}\right)\right\rangle \\
= & \left\langle v^{\prime}, \nu_{n}^{Y}\left(\left(z_{\sigma^{-1}(1)}, \theta_{\sigma^{-1}(1)}\right), \ldots,\left(z_{\sigma^{-1}(n-1)}, \theta_{\sigma^{-1}(n-1)}\right) ;\left(A^{(0)}, M^{(0)}\right),\right.\right. \\
& \left(a^{\left(\sigma^{-1}(1)\right)}, A^{\left(\sigma^{-1}(1)\right)}, M^{\left(\sigma^{-1}(1)\right)}\right), \ldots,\left(a^{\left(\sigma^{-1}(n-1)\right)}, A^{\left(\sigma^{-1}(n-1)\right)}, M^{\left(\sigma^{-1}(n-1)\right)}\right), \\
& \left.\left.\left(a^{(n)}, A^{(n)}, M^{(n)}\right)\right)\left(v_{1} \otimes \cdots \otimes v_{n}\right)\right\rangle
\end{aligned}
$$




$$
\begin{aligned}
& =\iota_{\sigma^{-1}(1) \cdots \sigma^{-1}(n-1)}^{-1}\left\langle e^{-\sum_{j \in \mathbb{Z}_{+}}\left(A_{j}^{(0)} L^{\prime}(j)+M_{j-\frac{1}{2}}^{(0)} G^{\prime}\left(j-\frac{1}{2}\right)\right)} v^{\prime},\right. \\
& Y\left(e^{-\sum_{j \in \mathbb{Z}_{+}}\left(A_{j}^{\left(\sigma^{-1}(1)\right)} L(j)+M_{j-\frac{1}{2}}^{\left(\sigma^{-1}(1)\right)} G\left(j-\frac{1}{2}\right)\right)} \cdot\left(a^{\left(\sigma^{-1}(1)\right)}\right)^{-2 L(0)} \cdot v_{1},\right. \\
& \left.\left(x_{\sigma^{-1}(1)}, \varphi_{\sigma^{-1}(1)}\right)\right) \cdots Y\left(e^{-\sum_{j \in \mathbb{Z}_{+}}\left(A_{j}^{\left(\sigma^{-1}(n-1)\right)} L(j)+M_{j-\frac{1}{2}}^{\left(\sigma^{-1}(n-1)\right)} G\left(j-\frac{1}{2}\right)\right)}\right. \text {. } \\
& \left.\left(a^{\left(\sigma^{-1}(n-1)\right)}\right)^{-2 L(0)} \cdot v_{n-1},\left(x_{\sigma^{-1}(n-1)}, \varphi_{\sigma^{-1}(n-1)}\right)\right) . \\
& \left.e^{-\sum_{j \in \mathbb{Z}_{+}}\left(A_{j}^{(n)} L(j)+M_{j-\frac{1}{2}}^{(n)} G\left(j-\frac{1}{2}\right)\right)} \cdot\left(a^{(n)}\right)^{-2 L(0)} \cdot v_{n}\right\rangle\left.\right|_{\left(x_{i}, \varphi_{i}\right)=\left(z_{i}, \theta_{i}\right)} \\
& =(-1)^{\eta(\sigma)} \iota_{1 \cdots n-1}^{-1}\left\langle e^{-\sum_{j \in \mathbb{Z}_{+}}\left(A_{j}^{(0)} L^{\prime}(j)+M_{j-\frac{1}{2}}^{(0)} G^{\prime}\left(j-\frac{1}{2}\right)\right)} v^{\prime},\right. \\
& Y\left(e^{-\sum_{j \in \mathbb{Z}_{+}}\left(A_{j}^{(1)} L(j)+M_{j-\frac{1}{2}}^{(1)} G\left(j-\frac{1}{2}\right)\right)} \cdot\left(a^{(1)}\right)^{-2 L(0)} \cdot v_{\sigma(1)},\left(x_{1}, \varphi_{1}\right)\right) \cdots \\
& Y\left(e^{-\sum_{j \in \mathbb{Z}_{+}}\left(A_{j}^{(n-1)} L(j)+M_{j-\frac{1}{2}}^{(n-1)} G\left(j-\frac{1}{2}\right)\right)} \cdot\left(a^{(n-1)}\right)^{-2 L(0)} \cdot v_{\sigma(n-1)},\right. \\
& \left.\left(x_{n-1}, \varphi_{n-1}\right)\right) e^{-\sum_{j \in \mathbb{Z}_{+}}\left(A_{j}^{(n)} L(j)+M_{j-\frac{1}{2}}^{(n)} G\left(j-\frac{1}{2}\right)\right)} . \\
& \left.\left(a^{(n)}\right)^{-2 L(0)} \cdot v_{n}\right\rangle\left.\right|_{\left(x_{i}, \varphi_{i}\right)=\left(z_{i}, \theta_{i}\right)} \\
& =(-1)^{\eta(\sigma)}\left\langle v^{\prime}, \nu_{n}^{Y}\left(\left(z_{1}, \theta_{1}\right), \ldots,\left(z_{n-1}, \theta_{n-1}\right) ;\left(A^{(0)}, M^{(0)}\right),\left(a^{(1)}, A^{(1)}, M^{(1)}\right), \ldots,\right.\right. \\
& \left.\left.\left(a^{(n)}, A^{(n)}, M^{(n)}\right)\right)\left(v_{\sigma(1)} \otimes \cdots \otimes v_{\sigma(n-1)} \otimes v_{n}\right)\right\rangle \\
& =\left\langle v^{\prime}, \nu_{n}^{Y}\left(\left(z_{1}, \theta_{1}\right), \ldots,\left(z_{n-1}, \theta_{n-1}\right) ;\left(A^{(0)}, M^{(0)}\right),\left(a^{(1)}, A^{(1)}, M^{(1)}\right), \ldots,\right.\right. \\
& \left.\left.\left(a^{(n)}, A^{(n)}, M^{(n)}\right)\right)\left(\left(v_{1} \otimes \cdots \otimes v_{n}\right) \cdot \sigma\right)\right\rangle \\
& =\left\langle v^{\prime}, \sigma \cdot\left(\nu_{n}^{Y}(Q)\right)\left(v_{1} \otimes \cdots \otimes v_{n}\right)\right\rangle
\end{aligned}
$$

which proves the permutation axiom for $\sigma \in S_{n-1} \subset S_{n}$. For the transposition switching $n$ and $n-1$, i.e., for $(n n-1) \in S_{n}$, using skew-supersymmetry (5.20),

$$
Y(u,(x, \varphi)) v=(-1)^{\eta(u) \eta(v)} e^{x L(-1)+\varphi G\left(-\frac{1}{2}\right)} Y(v,(-x,-\varphi)) u,
$$

we have

$$
\begin{aligned}
& \left\langle v^{\prime},(n n-1) \cdot\left(\nu_{n}^{Y}(Q)\right)\left(v_{1} \otimes \cdots \otimes v_{n}\right)\right\rangle \\
& =(-1)^{\eta\left(v_{n}\right) \eta\left(v_{n-1}\right)}\left\langle v^{\prime}, \nu_{n}^{Y}(Q)\left(v_{1} \otimes \cdots \otimes v_{n-2} \otimes v_{n} \otimes v_{n-1}\right)\right\rangle \\
& =(-1)^{\eta\left(v_{n}\right) \eta\left(v_{n-1}\right)}\left\langle v^{\prime}, \nu_{n}^{Y}\left(\left(\left(z_{1}, \theta_{1}\right), \ldots,\left(z_{n-1}, \theta_{n-1}\right) ;\left(A^{(0)}, M^{(0)}\right),\right.\right.\right. \\
& \left.\left.\left.\quad\left(a^{(1)}, A^{(1)}, M^{(1)}\right), \ldots,\left(a^{(n)}, A^{(n)}, M^{(n)}\right)\right)\right)\left(v_{1} \otimes \cdots \otimes v_{n-2} \otimes v_{n} \otimes v_{n-1}\right)\right\rangle
\end{aligned}
$$




$$
\begin{gathered}
N=1 \text { SG-VOSAS } \\
=(-1)^{\eta\left(v_{n}\right) \eta\left(v_{n-1}\right)} \iota_{1 \cdots n-1}^{-1}\left\langle e^{-\sum_{j \in \mathbb{Z}_{+}}\left(A_{j}^{(0)} L^{\prime}(j)+M_{j-\frac{1}{2}}^{(0)} G^{\prime}\left(j-\frac{1}{2}\right)\right.}\right) v^{\prime}, \\
Y\left(e^{-\sum_{j \in \mathbb{Z}_{+}}\left(A_{j}^{(1)} L(j)+M_{j-\frac{1}{2}}^{(1)} G\left(j-\frac{1}{2}\right)\right)} \cdot\left(a^{(1)}\right)^{-2 L(0)} \cdot v_{1},\left(x_{1}, \varphi_{1}\right)\right) \cdots \\
Y\left(e^{-\sum_{j \in \mathbb{Z}_{+}}\left(A_{j}^{(n-1)} L(j)+M_{j-\frac{1}{2}}^{(n-1)} G\left(j-\frac{1}{2}\right)\right)} \cdot\left(a^{(n-1)}\right)^{-2 L(0)} \cdot v_{n},\left(x_{n-1}, \varphi_{n-1}\right)\right) . \\
\left.e^{-\sum_{j \in \mathbb{Z}_{+}}\left(A_{j}^{(n)} L(j)+M_{j-\frac{1}{2}}^{(n)} G\left(j-\frac{1}{2}\right)\right)} \cdot\left(a^{(n)}\right)^{-2 L(0)} \cdot v_{n-1}\right\rangle\left.\right|_{\left(x_{i}, \varphi_{i}\right)=\left(z_{i}, \theta_{i}\right)} \\
=\iota_{1 \cdots n-1}^{-1}\left\langle e^{-\sum_{j \in \mathbb{Z}_{+}}\left(A_{j}^{(0)} L^{\prime}(j)+M_{j-\frac{1}{2}}^{(0)} G^{\prime}\left(j-\frac{1}{2}\right)\right)} v^{\prime},\right. \\
Y\left(e^{-\sum_{j \in \mathbb{Z}_{+}}\left(A_{j}^{(1)} L(j)+M_{j-\frac{1}{2}}^{(1)} G\left(j-\frac{1}{2}\right)\right)} \cdot\left(a^{(1)}\right)^{-2 L(0)} \cdot v_{1},\left(x_{1}, \varphi_{1}\right)\right) \cdots \\
e^{x_{n-1} L(-1)+\varphi_{n-1} G\left(-\frac{1}{2}\right)} Y\left(e^{-\sum_{j \in \mathbb{Z}_{+}}\left(A_{j}^{(n)} L(j)+M_{j-\frac{1}{2}}^{(n)} G\left(j-\frac{1}{2}\right)\right)} \cdot\left(a^{(n)}\right)^{-2 L(0)} .\right. \\
\left.v_{n-1},\left(-x_{n-1},-\varphi_{n-1}\right)\right) \cdot e^{-\sum_{j \in \mathbb{Z}_{+}}\left(A_{j}^{(n-1)} L(j)+M_{j-\frac{1}{2}}^{(n-1)} G\left(j-\frac{1}{2}\right)\right)} . \\
\left.\left(a^{(n-1)}\right)^{-2 L(0)} \cdot v_{n}\right\rangle\left.\right|_{\left(x_{i}, \varphi_{i}\right)=\left(z_{i}, \theta_{i}\right)} .
\end{gathered}
$$

Using equations (5.18) and (5.19), the right-hand side of (7.4) is equal to

$$
\begin{aligned}
& \iota_{1 \cdots n-1}^{-1}\left\langle e^{-\sum_{j \in \mathbb{Z}_{+}}\left(A_{j}^{(0)} L^{\prime}(j)+M_{j-\frac{1}{2}}^{(0)} G^{\prime}\left(j-\frac{1}{2}\right)\right)} v^{\prime}, e^{x_{n-1} L(-1)+\varphi_{n-1} G\left(-\frac{1}{2}\right)} .\right. \\
& Y\left(e^{-\sum_{j \in \mathbb{Z}_{+}}\left(A_{j}^{(1)} L(j)+M_{j-\frac{1}{2}}^{(1)} G\left(j-\frac{1}{2}\right)\right)} \cdot\left(a^{(1)}\right)^{-2 L(0)} \cdot v_{1}, s_{\left(x_{n-1}, \varphi_{n-1}\right)}\left(x_{1}, \varphi_{1}\right)\right) . \\
& \cdots Y\left(e^{-\sum_{j \in \mathbb{Z}_{+}}\left(A_{j}^{(n-2)} L(j)+M_{j-\frac{1}{2}}^{(n-2)} G\left(j-\frac{1}{2}\right)\right)} \cdot\left(a^{(n-2)}\right)^{-2 L(0)} v_{n-2},\right. \\
& \left.s_{\left(x_{n-1}, \varphi_{n-1}\right)}\left(x_{n-2}, \varphi_{n-2}\right)\right) . \\
& Y\left(e^{-\sum_{j \in \mathbb{Z}_{+}}\left(A_{j}^{(n)} L(j)+M_{j-\frac{1}{2}}^{(n)} G\left(j-\frac{1}{2}\right)\right)} \cdot\left(a^{(n)}\right)^{-2 L(0)} \cdot v_{n-1},\left(-x_{n-1},-\varphi_{n-1}\right)\right) . \\
& \left.e^{-\sum_{j \in \mathbb{Z}_{+}}\left(A_{j}^{(n-1)} L(j)+M_{j-\frac{1}{2}}^{(n-1)} G\left(j-\frac{1}{2}\right)\right)} \cdot\left(a^{(n-1)}\right)^{-2 L(0)} \cdot v_{n}\right\rangle\left.\right|_{\left(x_{i}, \varphi_{i}\right)=\left(z_{i}, \theta_{i}\right)} \\
& =\iota_{1 \cdots n-1}^{-1}\left\langle e^{x_{n-1} L^{\prime}(1)+\varphi_{n-1} G^{\prime}\left(\frac{1}{2}\right)} \cdot e^{-\sum_{j \in \mathbb{Z}_{+}}\left(A_{j}^{(0)} L^{\prime}(j)+M_{j-\frac{1}{2}}^{(0)} G^{\prime}\left(j-\frac{1}{2}\right)\right)} v^{\prime},\right. \\
& Y\left(e^{-\sum_{j \in \mathbb{Z}_{+}}\left(A_{j}^{(1)} L(j)+M_{j-\frac{1}{2}}^{(1)} G\left(j-\frac{1}{2}\right)\right)} \cdot\left(a^{(1)}\right)^{-2 L(0)} \cdot v_{1}, s_{\left(x_{n-1}, \varphi_{n-1}\right)}\left(x_{1}, \varphi_{1}\right)\right) . \\
& \cdots Y\left(e^{-\sum_{j \in \mathbb{Z}_{+}}\left(A_{j}^{(n-2)} L(j)+M_{j-\frac{1}{2}}^{(n-2)} G\left(j-\frac{1}{2}\right)\right)} \cdot\left(a^{(n-2)}\right)^{-2 L(0)} v_{n-2},\right. \\
& \left.s_{\left(x_{n-1}, \varphi_{n-1}\right)}\left(x_{n-2}, \varphi_{n-2}\right)\right) . \\
& Y\left(e^{-\sum_{j \in \mathbb{Z}_{+}}\left(A_{j}^{(n)} L(j)+M_{j-\frac{1}{2}}^{(n)} G\left(j-\frac{1}{2}\right)\right)} \cdot\left(a^{(n)}\right)^{-2 L(0)} \cdot v_{n-1},\left(-x_{n-1},-\varphi_{n-1}\right)\right) . \\
& \left.e^{-\sum_{j \in \mathbb{Z}_{+}}\left(A_{j}^{(n-1)} L(j)+M_{j-\frac{1}{2}}^{(n-1)} G\left(j-\frac{1}{2}\right)\right)} \cdot\left(a^{(n-1)}\right)^{-2 L(0)} \cdot v_{n}\right\rangle\left.\right|_{\left(x_{i}, \varphi_{i}\right)=\left(z_{i}, \theta_{i}\right)} .
\end{aligned}
$$


From (2.12) and the definition of $\nu^{Y}$, the right-hand side of (7.5) is equal to

$$
\begin{aligned}
& \iota_{1 \cdots n-1}^{-1}\left\langle e^{-\sum_{j \in \mathbb{Z}_{+}}\left(\tilde{A}_{j}^{(0)} L^{\prime}(j)+\tilde{M}_{j-\frac{1}{2}}^{(0)} G^{\prime}\left(j-\frac{1}{2}\right)\right)} v^{\prime}, Y\left(e^{-\sum_{j \in \mathbb{Z}_{+}}\left(A_{j}^{(1)} L(j)+M_{j-\frac{1}{2}}^{(1)} G\left(j-\frac{1}{2}\right)\right)} .\right.\right. \\
& \left.\left(a^{(1)}\right)^{-2 L(0)} \cdot v_{1}, s_{\left(x_{n-1}, \varphi_{n-1}\right)}\left(x_{1}, \varphi_{1}\right)\right) \cdots Y\left(e^{-\sum_{j \in \mathbb{Z}_{+}}\left(A_{j}^{(n-2)} L(j)+M_{j-\frac{1}{2}}^{(n-2)} G\left(j-\frac{1}{2}\right)\right)} .\right. \\
& \left.\cdot\left(a^{(n-2)}\right)^{-2 L(0)} \cdot v_{n-2}, s_{\left(x_{n-1}, \varphi_{n-1}\right)}\left(x_{n-2}, \varphi_{n-2}\right)\right) \cdot \\
& Y\left(e^{-\sum_{j \in \mathbb{Z}_{+}}\left(A_{j}^{(n)} L(j)+M_{j-\frac{1}{2}}^{(n)} G\left(j-\frac{1}{2}\right)\right)} \cdot\left(a^{(n)}\right)^{-2 L(0)} \cdot v_{n-1},\left(-x_{n-1},-\varphi_{n-1}\right)\right) . \\
& \left.e^{-\sum_{j \in \mathbb{Z}_{+}}\left(A_{j}^{(n-1)} L(j)+M_{j-\frac{1}{2}}^{(n-1)} G\left(j-\frac{1}{2}\right)\right)} \cdot\left(a^{(n-1)}\right)^{-2 L(0)} \cdot v_{n}\right\rangle\left.\right|_{\left(x_{i}, \varphi_{i}\right)=\left(z_{i}, \theta_{i}\right)} \\
& =\left\langle v^{\prime}, \nu_{n}^{Y}\left(s_{\left(z_{n-1}, \theta_{n-1}\right)}\left(z_{1}, \theta_{1}\right), s_{\left(z_{n-1}, \theta_{n-1}\right)}\left(z_{2}, \theta_{2}\right), \ldots, s_{\left(z_{n-1}, \theta_{n-1}\right)}\left(z_{n-2}, \theta_{n-2}\right),\right.\right. \\
& s_{\left(z_{n-1}, \theta_{n-1}\right)}(0) ;\left(\tilde{A}^{(0)}, \tilde{M}^{(0)}\right),\left(a^{(1)}, A^{(1)}, M^{(1)}\right), \ldots,\left(a^{(n-2)}, A^{(n-2)}, M^{(n-2)}\right), \\
& \left.\left.\left(a^{(n)}, A^{(n)}, M^{(n)}\right),\left(a^{(n-1)}, A^{(n-1)}, M^{(n-1)}\right)\right)\left(v_{1} \otimes \cdots \otimes v_{n}\right)\right\rangle \\
& =\left\langle v^{\prime}, \nu_{n}^{Y}\left(( n n - 1 ) \cdot \left(\left(z_{1}, \theta_{1}\right), \ldots,\left(z_{n-1}, \theta_{n-1}\right) ;\left(A^{(0)}, M^{(0)}\right),\left(a^{(1)}, A^{(1)}, M^{(1)}\right),\right.\right.\right. \\
& \left.\left.\left.\ldots,\left(a^{(n)}, A^{(n)}, M^{(n)}\right)\right)\right)\left(v_{1} \otimes \cdots \otimes v_{n}\right)\right\rangle \\
& (7.6)=\left\langle v^{\prime}, \nu_{n}^{Y}((n n-1) \cdot Q)\left(v_{1} \otimes \cdots \otimes v_{n}\right)\right\rangle .
\end{aligned}
$$

Combining (7.4), (7.5), and (7.6), we see that the permutation axiom is true for the transposition $(n n-1) \in S_{n}$. Since $S_{n}$ is generated by $S_{n-1}$ and $(n n-1)$, the permutation axiom holds for arbitrary $\sigma \in S_{n}$.

(5) Sewing axiom: Suppose $Q_{1} \in S K(m)$ and $Q_{2} \in S K(n)$ are given by

$$
\begin{gathered}
Q_{1}=\left(\left(z_{1}, \theta_{1}\right), \ldots,\left(z_{m-1}, \theta_{m-1}\right) ;\left(A^{(0)}, M^{(0)}\right),\left(a^{(1)}, A^{(1)}, M^{(1)}\right),\right. \\
\left.\ldots,\left(a^{(m)}, A^{(m)}, M^{(m)}\right)\right), \\
Q_{2}=\left(\left(z_{1}^{\prime}, \theta_{1}^{\prime}\right), \ldots,\left(z_{n-1}^{\prime}, \theta_{n-1}^{\prime}\right) ;\left(B^{(0)}, N^{(0)}\right),\left(b^{(1)}, B^{(1)}, N^{(1)}\right),\right. \\
\left.\ldots,\left(b^{(n)}, B^{(n)}, N^{(n)}\right)\right)
\end{gathered}
$$

and the $i$-th tube of $Q_{1}$, for some $1 \leq i \leq m$, can be sewn with the 0 -th tube of $Q_{2}$. We show by induction on $m$ and $n$ that the sewing axiom holds for $Q_{1}$ and $Q_{2}$. The proof is in eight steps:

(a) The case $m=1, n=0,1$.

(b) - (f) Several special cases needed for the induction.

(g) Induction on $n$.

(h) Induction on $m$.

We start with step (a): We prove the case $m=1, n=0,1$ by considering

$$
\begin{aligned}
& Q_{1}=\left(\left(A^{(0)}, M^{(0)}\right),\left(a^{(1)}, A^{(1)}, M^{(1)}\right)\right) \in S K(1) \\
& Q_{2}=\left(\left(B^{(0)}, N^{(0)}\right),\left(b^{(1)}, B^{(1)}, N^{(1)}\right)\right) \in S K(1)
\end{aligned}
$$

where $Q_{1}{ }_{1} \infty_{0} Q_{2}$ exists. Using the bases (4.2) and (4.3), we have $\left\langle v^{\prime},\left(\nu_{1}^{Y}\left(Q_{1}\right)_{1 *_{0}} \nu_{1}^{Y}\left(Q_{2}\right)\right)_{t^{1 / 2}}(v)\right\rangle$ 


$$
\begin{aligned}
& =\sum_{k \in \frac{1}{2} \mathbb{Z}} \sum_{i(k)=1}^{\operatorname{dim} V_{(k)}}\left\langle e^{-\sum_{j \in \mathbb{Z}_{+}}\left(A_{j}^{(0)} L^{\prime}(j)+M_{j-\frac{1}{2}}^{(0)} G^{\prime}\left(j-\frac{1}{2}\right)\right)} v^{\prime},\right. \\
& \left.e^{-\sum_{j \in \mathbb{Z}_{+}}\left(A_{j}^{(1)} L(j)+M_{j-\frac{1}{2}}^{(1)} G\left(j-\frac{1}{2}\right)\right)} \cdot\left(a^{(1)}\right)^{-2 L(0)} \cdot e_{i^{(k)}}^{(k)}\right\rangle \cdot \\
& \left\langle e^{-\sum_{j \in \mathbb{Z}_{+}}\left(B_{j}^{(0)} L^{\prime}(j)+N_{j-\frac{1}{2}}^{(0)} G^{\prime}\left(j-\frac{1}{2}\right)\right)}\left(e_{i(k)}^{(k)}\right)^{*},\right. \\
& \left.e^{-\sum_{j \in \mathbb{Z}_{+}}\left(B_{j}^{(1)} L(j)+N_{j-\frac{1}{2}}^{(1)} G\left(j-\frac{1}{2}\right)\right)} \cdot\left(b^{(1)}\right)^{-2 L(0)} \cdot v\right\rangle t^{k} \\
& =\sum_{k \in \frac{1}{2} \mathbb{Z}} \sum_{i^{(k)}=1}^{\operatorname{dim} V_{(k)}}\left\langle e^{-\sum_{j \in \mathbb{Z}_{+}}\left(A_{j}^{(0)} L^{\prime}(j)+M_{j-\frac{1}{2}}^{(0)} G^{\prime}\left(j-\frac{1}{2}\right)\right)} v^{\prime},\right. \\
& \left.e^{-\sum_{j \in \mathbb{Z}_{+}}\left(A_{j}^{(1)} L(j)+M_{j-\frac{1}{2}}^{(1)} G\left(j-\frac{1}{2}\right)\right)} \cdot\left(a^{(1)}\right)^{-2 L(0)} \cdot e_{i^{(k)}}^{(k)}\right\rangle \cdot \\
& \left\langle e^{-\sum_{j \in \mathbb{Z}_{+}}\left(B_{j}^{(0)} L^{\prime}(j)+N_{j-\frac{1}{2}}^{(0)} G^{\prime}\left(j-\frac{1}{2}\right)\right)} \cdot t^{L^{\prime}(0)}\left(e_{i(k)}^{(k)}\right)^{*},\right. \\
& \left.e^{-\sum_{j \in \mathbb{Z}_{+}}\left(B_{j}^{(1)} L(j)+N_{j-\frac{1}{2}}^{(1)} G\left(j-\frac{1}{2}\right)\right)} \cdot\left(b^{(1)}\right)^{-2 L(0)} \cdot v\right\rangle \\
& =\sum_{k \in \frac{1}{2} \mathbb{Z}} \sum_{i^{(k)}=1}^{\operatorname{dim} V_{(k)}}\left\langle e^{-\sum_{j \in \mathbb{Z}_{+}}\left(A_{j}^{(0)} L^{\prime}(j)+M_{j-\frac{1}{2}}^{(0)} G^{\prime}\left(j-\frac{1}{2}\right)\right)} v^{\prime},\right. \\
& \left.e^{-\sum_{j \in \mathbb{Z}_{+}}\left(A_{j}^{(1)} L(j)+M_{j-\frac{1}{2}}^{(1)} G\left(j-\frac{1}{2}\right)\right)} \cdot\left(a^{(1)}\right)^{-2 L(0)} \cdot e_{i^{(k)}}^{(k)}\right\rangle \cdot \\
& \left\langle\left(e_{i(k)}^{(k)}\right)^{*}, t^{L(0)} \cdot e^{-\sum_{j \in \mathbb{Z}_{+}}\left(B_{j}^{(0)} L(-j)+N_{j-\frac{1}{2}}^{(0)} G\left(-j+\frac{1}{2}\right)\right)} .\right. \\
& \left.e^{-\sum_{j \in \mathbb{Z}_{+}}\left(B_{j}^{(1)} L(j)+N_{j-\frac{1}{2}}^{(1)} G\left(j-\frac{1}{2}\right)\right)} \cdot\left(b^{(1)}\right)^{-2 L(0)} \cdot v\right\rangle \\
& =\left\langle e^{-\sum_{j \in \mathbb{Z}_{+}}\left(A_{j}^{(0)} L^{\prime}(j)+M_{j-\frac{1}{2}}^{(0)} G^{\prime}\left(j-\frac{1}{2}\right)\right)} v^{\prime}, e^{-\sum_{j \in \mathbb{Z}_{+}}\left(A_{j}^{(1)} L(j)+M_{j-\frac{1}{2}}^{(1)} G\left(j-\frac{1}{2}\right)\right)} .\right. \\
& \left(t^{\frac{1}{2}} a^{(1)}\right)^{-2 L(0)} \cdot e^{-\sum_{j \in \mathbb{Z}_{+}}\left(B_{j}^{(0)} L(-j)+N_{j-\frac{1}{2}}^{(0)} G\left(-j+\frac{1}{2}\right)\right)} . \\
& \left.e^{-\sum_{j \in \mathbb{Z}_{+}}\left(B_{j}^{(1)} L(j)+N_{j-\frac{1}{2}}^{(1)} G\left(j-\frac{1}{2}\right)\right)} \cdot\left(b^{(1)}\right)^{-2 L(0)} \cdot v\right\rangle .
\end{aligned}
$$

Let $\Psi_{j}\left(t^{1 / 2}\right)=\Psi_{j}\left(t^{-1 / 2} a^{(1)}, A^{(1)}, M^{(1)}, B^{(0)}, N^{(0)}\right)$, for $j \in \frac{1}{2} \mathbb{Z}$, and let $\Gamma\left(t^{1 / 2}\right)=$ $\Gamma\left(t^{-1 / 2} a^{(1)}, A^{(1)}, M^{(1)}, B^{(0)}, N^{(0)}\right)$. From Corollary 3.4 equation (7.7) is equal to

$$
\begin{array}{r}
\left\langle e^{-\sum_{j \in \mathbb{Z}_{+}}\left(A_{j}^{(0)} L^{\prime}(j)+M_{j-\frac{1}{2}}^{(0)} G^{\prime}\left(j-\frac{1}{2}\right)\right)} v^{\prime}, e^{\sum_{j \in \mathbb{Z}_{+}}\left(\Psi_{-j}\left(t^{\frac{1}{2}}\right) L(-j)+\Psi_{-j+\frac{1}{2}}\left(t^{\frac{1}{2}}\right) G\left(-j+\frac{1}{2}\right)\right)}\right. \\
e^{\sum_{j \in \mathbb{Z}_{+}}\left(\Psi_{j}\left(t^{\frac{1}{2}}\right) L(j)+\Psi_{j-\frac{1}{2}}\left(t^{\frac{1}{2}}\right) G\left(j-\frac{1}{2}\right)\right)} e^{2 \Psi_{0}\left(t^{\frac{1}{2}}\right) L(0)}\left(t^{-\frac{1}{2}} a^{(1)}\right)^{-2 L(0)} e^{\Gamma\left(t^{\frac{1}{2}}\right) c} \\
\left.e^{-\sum_{j \in \mathbb{Z}_{+}}\left(B_{j}^{(1)} L(j)+N_{j-\frac{1}{2}}^{(1)} G\left(j-\frac{1}{2}\right)\right)} \cdot\left(b^{(1)}\right)^{-2 L(0)} \cdot v\right\rangle
\end{array}
$$




$$
\begin{gathered}
=\left\langle e^{\sum_{j \in \mathbb{Z}_{+}}\left(\Psi_{-j}\left(t^{\frac{1}{2}}\right) L^{\prime}(j)+\Psi_{-j+\frac{1}{2}}\left(t^{\frac{1}{2}}\right) G^{\prime}\left(j-\frac{1}{2}\right)\right)} \cdot e^{-\sum_{j \in \mathbb{Z}_{+}}\left(A_{j}^{(0)} L^{\prime}(j)+M_{j-\frac{1}{2}}^{(0)} G^{\prime}\left(j-\frac{1}{2}\right)\right)} v^{\prime},\right. \\
e^{\sum_{j \in \mathbb{Z}_{+}}\left(\Psi_{j}\left(t^{\frac{1}{2}}\right) L(j)+\Psi_{j-\frac{1}{2}}\left(t^{\frac{1}{2}}\right) G\left(j-\frac{1}{2}\right)\right)} e^{2 \Psi_{0}\left(t^{\frac{1}{2}}\right) L(0)}\left(t^{-\frac{1}{2}} a^{(1)}\right)^{-2 L(0)} \\
\left.e^{-\sum_{j \in \mathbb{Z}_{+}}\left(B_{j}^{(1)} L(j)+N_{j-\frac{1}{2}}^{(1)} G\left(j-\frac{1}{2}\right)\right)} \cdot\left(b^{(1)}\right)^{-2 L(0)} \cdot v\right\rangle e^{\Gamma\left(t^{\frac{1}{2}}\right) c}
\end{gathered}
$$

Thus

$$
\begin{aligned}
& \left\langle v^{\prime},\left(\nu_{1}^{Y}\left(Q_{1}\right){ }_{1} *_{0} \nu_{1}^{Y}\left(Q_{2}\right)\right)_{t^{1 / 2}}(v)\right\rangle e^{-\Gamma\left(t^{\frac{1}{2}}\right) c} \\
& \quad=\left\langle e^{\sum_{j \in \mathbb{Z}_{+}}\left(\Psi_{-j}\left(t^{\frac{1}{2}}\right) L^{\prime}(j)+\Psi_{-j+\frac{1}{2}}\left(t^{\frac{1}{2}}\right) G^{\prime}\left(j-\frac{1}{2}\right)\right)} \cdot e^{-\sum_{j \in \mathbb{Z}_{+}}\left(A_{j}^{(0)} L^{\prime}(j)+M_{j-\frac{1}{2}}^{(0)} G^{\prime}\left(j-\frac{1}{2}\right)\right)} v^{\prime},\right. \\
& \quad e^{\sum_{j \in \mathbb{Z}_{+}}\left(\Psi_{j}\left(t^{\frac{1}{2}}\right) L(j)+\Psi_{j-\frac{1}{2}}\left(t^{\frac{1}{2}}\right) G\left(j-\frac{1}{2}\right)\right)} e^{2 \Psi_{0}\left(t^{\frac{1}{2}}\right) L(0)}\left(t^{-\frac{1}{2}} a^{(1)}\right)^{-2 L(0)} . \\
& \left.\quad e^{-\sum_{j \in \mathbb{Z}_{+}}\left(B_{j}^{(1)} L(j)+N_{j-\frac{1}{2}}^{(1)} G\left(j-\frac{1}{2}\right)\right)} \cdot\left(b^{(1)}\right)^{-2 L(0)} \cdot v\right\rangle .
\end{aligned}
$$

Since $V$ is a positive energy representation of $\mathfrak{n s}$, the right-hand side of (7.8) is a polynomial in $\Psi_{j}\left(t^{1 / 2}\right)$, for $j \in \frac{1}{2} \mathbb{Z}, j \neq 0$, and $e^{ \pm 2 \Psi_{0}\left(t^{1 / 2}\right)}$ with coefficients in $\bigwedge_{\infty}\left[t^{1 / 2}, t^{-1 / 2}\right]$. By Theorem 2.3 the series $\Psi_{j}\left(t^{1 / 2}\right)$ and $e^{ \pm 2 \tilde{\Psi}_{0}\left(t^{1 / 2}\right)}$ converge to $\tilde{\Psi}_{j}\left(a^{(1)}, A^{(1)}, M^{(1)}, B^{(0)}, N^{(0)}\right)$ and $e^{ \pm 2 \tilde{\Psi}_{0}\left(a^{(1)}, A^{(1)}, M^{(1)}, B^{(0)}, N^{(0)}\right)}$, respectively, at $t^{1 / 2}=1$. Let $\Psi_{j}=\Psi_{j}\left(a^{(1)}, A^{(1)}, M^{(1)}, B^{(0)}, N^{(0)}\right)$ for $j \in \frac{1}{2} \mathbb{Z}$. From equations (2.22) and (2.23), Proposition 3.21 of [B4], and Theorem [2.3] the local coordinate at $\infty$ of the canonical supersphere with $1+1$ tubes representing $Q_{1}{ }_{1} \infty_{0} Q_{2}$, in terms of the superderivation representation of $\mathfrak{n} \mathfrak{s}$, (2.1) and (2.2), is given by

$$
\begin{aligned}
& \exp \left(-\sum_{j \in \mathbb{Z}_{+}}\left(\Psi_{-j} L_{-j}(w, \rho)+\Psi_{-j+\frac{1}{2}} G_{-j+\frac{1}{2}}(w, \rho)\right)\right) \cdot \\
& \quad \exp \left(\sum_{j \in \mathbb{Z}_{+}}\left(A_{j}^{(0)} L_{-j}(w, \rho)+M_{j-\frac{1}{2}}^{(0)} G_{-j+\frac{1}{2}}(w, \rho)\right)\right) \cdot\left(\frac{1}{w}, \frac{i \rho}{w}\right),
\end{aligned}
$$

and the local coordinate at zero is given by

$$
\begin{aligned}
\exp (- & \left.\sum_{j \in \mathbb{Z}_{+}}\left(\Psi_{j} L_{j}(w, \rho)+\Psi_{j-\frac{1}{2}} G_{j-\frac{1}{2}}(w, \rho)\right)\right) \cdot\left(a^{(1)}\right)^{-L_{0}(w, \rho)} \cdot e^{\Psi_{0} L_{0}(w, \rho)} . \\
& \exp \left(-\sum_{j \in \mathbb{Z}_{+}}\left(B_{j}^{(1)} L_{j}(w, \rho)+N_{j-\frac{1}{2}}^{(1)} G_{j-\frac{1}{2}}(w, \rho)\right)\right) \cdot\left(b^{(1)}\right)^{-L_{0}(w, \rho)} \cdot(w, \rho) .
\end{aligned}
$$

By Proposition 3.17 of [B4] and by Proposition 2.1] there exist series $\left(C^{(0)}, O^{(0)}\right)$, $\left(C^{(1)}, O^{(1)}\right) \in \bigwedge_{\infty}^{\infty}$ and $c^{(1)} \in\left(\bigwedge_{\infty}^{0}\right)^{\times}$such that these local coordinates can be written as

$$
\exp \left(\sum_{j \in \mathbb{Z}_{+}}\left(C_{j}^{(0)} L_{-j}(w, \rho)+O_{j-\frac{1}{2}}^{(0)} G_{-j+\frac{1}{2}}(w, \rho)\right)\right) \cdot\left(\frac{1}{w}, \frac{i \rho}{w}\right)
$$

and

$$
\exp \left(-\sum_{j \in \mathbb{Z}_{+}}\left(C_{j}^{(1)} L_{j}(w, \rho)+O_{j-\frac{1}{2}}^{(1)} G_{j-\frac{1}{2}}(w, \rho)\right)\right) \cdot\left(c^{(1)}\right)^{-L_{0}(w, \rho)} \cdot(w, \rho),
$$


respectively. Since $V$ is a positive-energy representation of $\mathfrak{n s}$ and by Proposition 3.5 when $t^{1 / 2}=1$ the series $\Gamma\left(t^{-1 / 2} a^{(1)}, A^{(1)}, M^{(1)}, B^{(0)}, N^{(0)}\right)$ converges to $\Gamma\left(a^{(1)}, A^{(1)}, M^{(1)}, B^{(0)}, N^{(0)}\right)$, and we have

$$
\begin{aligned}
& \left\langle v^{\prime},\left(\nu_{1}^{Y}\left(Q_{1}\right){ }_{1} *_{0} \nu_{2}^{Y}\left(Q_{1}\right)\right)(v)\right\rangle e^{-\Gamma\left(a^{(1)}, A^{(1)}, M^{(1)}, B^{(0)}, N^{(0)}\right) c} \\
& =\left\langle e^{-\sum_{j \in \mathbb{Z}_{+}}\left(C_{j}^{(0)} L^{\prime}(j)+O_{j-\frac{1}{2}}^{(0)} G^{\prime}\left(j-\frac{1}{2}\right)\right)} v^{\prime},\right. \\
& \left.e^{-\sum_{j \in \mathbb{Z}_{+}}\left(C_{j}^{(1)} L(j)+O_{j-\frac{1}{2}}^{(1)} G\left(j-\frac{1}{2}\right)\right)} \cdot\left(c^{(1)}\right)^{-2 L(0)} v\right\rangle \\
& =\left\langle v^{\prime}, \nu_{1}^{Y}\left(Q_{1}{ }_{1} \infty_{0} Q_{2}\right)(v)\right\rangle
\end{aligned}
$$

which proves the sewing axiom for $n=1, m=1$. The case $m=1, n=0$ is then given by letting $\left(b^{(1)}, B^{(1)}, N^{(1)}\right)=(1, \mathbf{0})$ and $v=\mathbf{1}$ above.

(b) We prove the sewing axiom for the special case $Q_{12} \infty_{0} Q_{2}$ when

$$
Q_{1}=\left((z, \theta) ; \mathbf{0},\left(a^{(1)}, A^{(1)}, M^{(1)}\right),(1, \mathbf{0})\right) \in S K(2)
$$

and

$$
Q_{2}=\left(\left(B^{(0)}, N^{(0)}\right),\left(b^{(1)}, B^{(1)}, N^{(1)}\right)\right) \in S K(1) .
$$

In this case,

$$
\begin{aligned}
& \left\langle v^{\prime},\left(\nu_{2}^{Y}\left(Q_{1}\right)_{2} *_{0} \nu_{1}^{Y}\left(Q_{2}\right)\right)_{t^{1 / 2}}\left(v_{1} \otimes v_{2}\right)\right\rangle \\
& =\sum_{k \in \frac{1}{2} \mathbb{Z}} \sum_{i^{(k)}=1}^{\operatorname{dim} V_{(k)}}\left\langle v^{\prime}, Y\left(e^{-\sum_{j \in \mathbb{Z}_{+}}\left(A_{j}^{(1)} L(j)+M_{j-\frac{1}{2}}^{(1)} G\left(j-\frac{1}{2}\right)\right)} \cdot\left(a^{(1)}\right)^{-2 L(0)} \cdot v_{1},\right.\right. \\
& \left.(x, \varphi)) e_{i(k)}^{(k)}\right\rangle \cdot\left\langle e^{-\sum_{j \in \mathbb{Z}_{+}}\left(B_{j}^{(0)} L^{\prime}(j)+N_{j-\frac{1}{2}}^{(0)} G^{\prime}\left(j-\frac{1}{2}\right)\right)} \cdot\left(e_{i(k)}^{(k)}\right)^{*},\right. \\
& \left.e^{-\sum_{j \in \mathbb{Z}_{+}}\left(B_{j}^{(1)} L(j)+N_{j-\frac{1}{2}}^{(1)} G\left(j-\frac{1}{2}\right)\right)} \cdot\left(b^{(1)}\right)^{-2 L(0)} \cdot v_{2}\right\rangle\left. t^{k}\right|_{(x, \varphi)=(z, \theta)} \\
& =\sum_{k \in \frac{1}{2} \mathbb{Z}} \sum_{i^{(k)}=1}^{\operatorname{dim} V_{(k)}}\left\langle v^{\prime}, Y\left(e^{-\sum_{j \in \mathbb{Z}_{+}}\left(A_{j}^{(1)} L(j)+M_{j-\frac{1}{2}}^{(1)} G\left(j-\frac{1}{2}\right)\right)} \cdot\left(a^{(1)}\right)^{-2 L(0)} \cdot v_{1},\right.\right. \\
& \left.(x, \varphi)) e_{i(k)}^{(k)}\right\rangle \cdot\left\langle e^{-\sum_{j \in \mathbb{Z}_{+}}\left(B_{j}^{(0)} L^{\prime}(j)+N_{j-\frac{1}{2}}^{(0)} G^{\prime}\left(j-\frac{1}{2}\right)\right)} \cdot t^{L^{\prime}(0)}\left(e_{i^{(k)}}^{(k)}\right)^{*},\right. \\
& \left.e^{-\sum_{j \in \mathbb{Z}_{+}}\left(B_{j}^{(1)} L(j)+N_{j-\frac{1}{2}}^{(1)} G\left(j-\frac{1}{2}\right)\right)} \cdot\left(b^{(1)}\right)^{-2 L(0)} \cdot v_{2}\right\rangle\left.\right|_{(x, \varphi)=(z, \theta)} \\
& =\sum_{k \in \frac{1}{2} \mathbb{Z}} \sum_{i(k)=1}^{\operatorname{dim} V_{(k)}}\left\langle v^{\prime}, Y\left(e^{-\sum_{j \in \mathbb{Z}_{+}}\left(A_{j}^{(1)} L(j)+M_{j-\frac{1}{2}}^{(1)} G\left(j-\frac{1}{2}\right)\right)} \cdot\left(a^{(1)}\right)^{-2 L(0)} \cdot v_{1},\right.\right. \\
& \left.(x, \varphi)) e_{i(k)}^{(k)}\right\rangle \cdot\left\langle t^{L^{\prime}(0)} \cdot e^{-\sum_{j \in \mathbb{Z}_{+}}\left(t^{j} B_{j}^{(0)} L^{\prime}(j)+t^{j-\frac{1}{2}} N_{j-\frac{1}{2}}^{(0)} G^{\prime}\left(j-\frac{1}{2}\right)\right)} \cdot\left(e_{i(k)}^{(k)}\right)^{*},\right. \\
& \left.e^{-\sum_{j \in \mathbb{Z}_{+}}\left(B_{j}^{(1)} L(j)+N_{j-\frac{1}{2}}^{(1)} G\left(j-\frac{1}{2}\right)\right)} \cdot\left(b^{(1)}\right)^{-2 L(0)} \cdot v_{2}\right\rangle\left.\right|_{(x, \varphi)=(z, \theta)}
\end{aligned}
$$




$$
\begin{aligned}
& =\sum_{k \in \frac{1}{2} \mathbb{Z}} \sum_{i^{(k)}=1}^{\operatorname{dim} V_{(k)}}\left\langle v^{\prime}, Y\left(e^{-\sum_{j \in \mathbb{Z}_{+}}\left(A_{j}^{(1)} L(j)+M_{j-\frac{1}{2}}^{(1)} G\left(j-\frac{1}{2}\right)\right)} \cdot\left(a^{(1)}\right)^{-2 L(0)} \cdot v_{1},\right.\right. \\
& \left.(x, \varphi)) e_{i(k)}^{(k)}\right\rangle \cdot\left\langle\left(e_{i(k)}^{(k)}\right)^{*}, e^{-\sum_{j \in \mathbb{Z}_{+}}\left(t^{j} B_{j}^{(0)} L(-j)+t^{j-\frac{1}{2}} N_{j-\frac{1}{2}}^{(0)} G\left(-j+\frac{1}{2}\right)\right)} \cdot t^{L(0) .}\right. \\
& \left.e^{-\sum_{j \in \mathbb{Z}_{+}}\left(B_{j}^{(1)} L(j)+N_{j-\frac{1}{2}}^{(1)} G\left(j-\frac{1}{2}\right)\right)} \cdot\left(b^{(1)}\right)^{-2 L(0)} \cdot v_{2}\right\rangle\left.\right|_{(x, \varphi)=(z, \theta)} \\
& =\left\langle v^{\prime}, Y\left(e^{-\sum_{j \in \mathbb{Z}_{+}}\left(A_{j}^{(1)} L(j)+M_{j-\frac{1}{2}}^{(1)} G\left(j-\frac{1}{2}\right)\right)} \cdot\left(a^{(1)}\right)^{-2 L(0)} \cdot v_{1},(x, \varphi)\right)\right. \\
& e^{-\sum_{j \in \mathbb{Z}_{+}}\left(t^{j} B_{j}^{(0)} L(-j)+t^{j-\frac{1}{2}} N_{j-\frac{1}{2}}^{(0)} G\left(-j+\frac{1}{2}\right)\right)} \cdot t^{L(0) .} \\
& \left.e^{-\sum_{j \in \mathbb{Z}_{+}}\left(B_{j}^{(1)} L(j)+N_{j-\frac{1}{2}}^{(1)} G\left(j-\frac{1}{2}\right)\right)} \cdot\left(b^{(1)}\right)^{-2 L(0)} \cdot v_{2}\right\rangle\left.\right|_{(x, \varphi)=(z, \theta)} \\
& =\left\langle e^{-\sum_{j \in \mathbb{Z}_{+}}\left(t^{j} B_{j}^{(0)} L^{\prime}(j)+t^{j-\frac{1}{2}} N_{j-\frac{1}{2}}^{(0)} G^{\prime}\left(j-\frac{1}{2}\right)\right)} \cdot v^{\prime},\right. \\
& e^{\sum_{j \in \mathbb{Z}_{+}}\left(t^{j} B_{j}^{(0)} L(-j)+t^{j-\frac{1}{2}} N_{j-\frac{1}{2}}^{(0)} G\left(-j+\frac{1}{2}\right)\right)} . \\
& Y\left(e^{-\sum_{j \in \mathbb{Z}_{+}}\left(A_{j}^{(1)} L(j)+M_{j-\frac{1}{2}}^{(1)} G\left(j-\frac{1}{2}\right)\right)} \cdot\left(a^{(1)}\right)^{-2 L(0)} \cdot v_{1},(x, \varphi)\right) \\
& e^{-\sum_{j \in \mathbb{Z}_{+}}\left(t^{j} B_{j}^{(0)} L(-j)+t^{j-\frac{1}{2}} N_{j-\frac{1}{2}}^{(0)} G\left(-j+\frac{1}{2}\right)\right)} \cdot t^{L(0)} . \\
& \left.e^{-\sum_{j \in \mathbb{Z}_{+}}\left(B_{j}^{(1)} L(j)+N_{j-\frac{1}{2}}^{(1)} G\left(j-\frac{1}{2}\right)\right)} \cdot\left(b^{(1)}\right)^{-2 L(0)} \cdot v_{2}\right\rangle\left.\right|_{(x, \varphi)=(z, \theta)} \cdot
\end{aligned}
$$

The right-hand side of (17.10) is a Laurent polynomial in $t^{1 / 2}$. If we can prove

$$
\begin{gathered}
e^{\sum_{j \in \mathbb{Z}_{+}}\left(t^{j} B_{j}^{(0)} L(-j)+t^{j-\frac{1}{2}} N_{j-\frac{1}{2}}^{(0)} G\left(-j+\frac{1}{2}\right)\right)} Y(u,(x, \varphi)) \\
e^{-\sum_{j \in \mathbb{Z}_{+}}\left(t^{j} B_{j}^{(0)} L(-j)+t^{j-\frac{1}{2}} N_{j-\frac{1}{2}}^{(0)} G\left(-j+\frac{1}{2}\right)\right)} \\
\quad=Y\left(e^{-\sum_{j \in \mathbb{Z}_{+}}\left(\Theta_{j}^{(2)} L(j)+\Theta_{j-\frac{1}{2}}^{(2)} G\left(j-\frac{1}{2}\right)\right)} e^{-2 \Theta_{0}^{(2)} L(0)} u, H_{t}^{-1} \circ I(x, \varphi)\right)
\end{gathered}
$$

where

$$
\Theta_{j}^{(2)}=\Theta_{j}^{(2)}\left(\left\{t^{k} B_{k}^{(0)}, t^{k-\frac{1}{2}} N_{k-\frac{1}{2}}^{(0)}\right\}_{k \in \mathbb{Z}_{+}},(x, \varphi)\right)
$$

is given by (3.8) and

$$
H_{t^{1 / 2}}^{-1}(x, \varphi)=\exp \left(\sum_{j \in \mathbb{Z}_{+}}\left(t^{j} B_{j}^{(0)} L_{-j}(x, \varphi)+t^{j-\frac{1}{2}} N_{j-\frac{1}{2}}^{(0)} G_{-j+\frac{1}{2}}(x, \varphi)\right)\right) \cdot\left(\frac{1}{x}, \frac{i \varphi}{x}\right)
$$

then when $t^{1 / 2}=1$, the right-hand side of (7.10) is equal to

$$
\left\langle v^{\prime}, \nu_{2}^{Y}\left(Q_{12} \infty_{0} Q_{2}\right)\left(v_{1} \otimes v_{2}\right)\right\rangle
$$


Thus in this case, the proof of the sewing axiom is reduced to the proof of (7.11). Letting

$$
H_{t^{1 / 2}}^{-1} \circ I(x, \varphi)=\left(\tilde{x}\left(t^{\frac{1}{2}}\right), \tilde{\varphi}\left(t^{\frac{1}{2}}\right)\right),
$$

by (5.17) and Corollary 3.9.

$$
\begin{gathered}
Y\left(e^{-\sum_{j \in \mathbb{Z}_{+}}\left(\Theta_{j}^{(2)} L(j)+\Theta_{j-\frac{1}{2}}^{(2)} G\left(j-\frac{1}{2}\right)\right)} e^{-2 \Theta_{0}^{(2)} L(0)} u, H_{t^{1 / 2}}^{-1} \circ I(x, \varphi)\right) \\
=Y\left(e^{\left(\tilde{x}\left(t^{\frac{1}{2}}\right)-x\right) L(-1)+\left(\tilde{\varphi}\left(t^{\frac{1}{2}}\right)-\varphi\right) G\left(-\frac{1}{2}\right)} \cdot e^{-\sum_{j \in \mathbb{Z}_{+}}\left(\Theta_{j}^{(2)} L(j)+\Theta_{j-\frac{1}{2}}^{(2)} G\left(j-\frac{1}{2}\right)\right)} .\right. \\
\left.e^{-2 \Theta_{0}^{(2)} L(0)} u,(x, \varphi)\right) \\
=Y\left(\operatorname { e x p } \left(\sum _ { m = - 1 } ^ { \infty } \sum _ { j \in \mathbb { Z } _ { + } } ( \begin{array} { c } 
{ - j + 1 } \\
{ m + 1 }
\end{array} ) x ^ { - j - m } \left(\left(t^{j} B_{j}^{(0)}+2 \varphi t^{j-\frac{1}{2}} N_{j-\frac{1}{2}}^{(0)}\right) L(m)\right.\right.\right. \\
\left.\left.\left.\quad+\left(t^{j-\frac{1}{2}} N_{j-\frac{1}{2}}^{(0)}+\varphi x^{-1} \frac{(-j-m)}{2} t^{j} B_{j}^{(0)}\right) G\left(m+\frac{1}{2}\right)\right)\right) u,(x, \varphi)\right) .
\end{gathered}
$$

By this equality, the proof of (7.11) is reduced to the proof of the following bracket formula

$$
\begin{array}{r}
{\left[\sum_{j \in \mathbb{Z}_{+}}\left(t^{j} B_{j}^{(0)} L(-j)+t^{j-\frac{1}{2}} N_{j-\frac{1}{2}}^{(0)} G\left(-j+\frac{1}{2}\right)\right), Y(u,(x, \varphi))\right]} \\
=Y\left(\sum _ { m = - 1 } ^ { \infty } \sum _ { j \in \mathbb { Z } _ { + } } ( \begin{array} { c } 
{ - j + 1 } \\
{ m + 1 }
\end{array} ) x ^ { - j - m } \left(\left(t^{j} B_{j}^{(0)}+2 \varphi t^{j-\frac{1}{2}} N_{j-\frac{1}{2}}^{(0)}\right) L(m)\right.\right. \\
\left.\left.+\left(t^{j-\frac{1}{2}} N_{j-\frac{1}{2}}^{(0)}+\varphi x^{-1} \frac{(-j-m)}{2} t^{j} B_{j}^{(0)}\right) G\left(m+\frac{1}{2}\right)\right) u,(x, \varphi)\right) .
\end{array}
$$

From (5.14), we have

$$
\begin{aligned}
& {\left[Y\left(\tau,\left(x_{1}, \varphi_{1}\right)\right), Y(u,(x, \varphi))\right]} \\
& \quad=\operatorname{Res}_{x_{0}} x^{-1} \delta\left(\frac{x_{1}-x_{0}-\varphi_{1} \varphi}{x}\right) Y\left(Y\left(\tau,\left(x_{0}, \varphi_{1}-\varphi\right)\right) u,(x, \varphi)\right) .
\end{aligned}
$$

Let

$$
l_{t}\left(x_{1}\right)=\sum_{j \in \mathbb{Z}_{+}} t^{j} B_{j}^{(0)} x_{1}^{-j+1} \quad \text { and } \quad g_{t}\left(x_{1}\right)=\sum_{j \in \mathbb{Z}_{+}} t^{j-\frac{1}{2}} N_{j-\frac{1}{2}}^{(0)} x_{1}^{-j+1}
$$

Then

$$
\begin{aligned}
\varphi_{1} \operatorname{Res}_{x_{1}} l_{t}\left(x_{1}\right) Y\left(\omega,\left(x_{1}, \varphi_{1}\right)\right) & =\varphi_{1} \sum_{j \in \mathbb{Z}_{+}} t^{j} B_{j}^{(0)} L(-j) \\
\varphi_{1} \operatorname{Res}_{x_{1}} g_{t}\left(x_{1}\right) Y\left(\tau,\left(x_{1}, \varphi_{1}\right)\right) & =\varphi_{1} \sum_{j \in \mathbb{Z}_{+}} t^{j-\frac{1}{2}} N_{j-\frac{1}{2}}^{(0)} G\left(-j+\frac{1}{2}\right),
\end{aligned}
$$


and by the $G(-1 / 2)$-derivative property (5.12), we have

$$
\begin{aligned}
Y\left(\omega,\left(x_{1}, \varphi_{1}\right)\right) & =Y\left(\frac{1}{2} G\left(-\frac{1}{2}\right) \tau,\left(x_{1}, \varphi_{1}\right)\right) \\
& =\frac{1}{2}\left(\frac{\partial}{\partial \varphi_{1}}+\varphi_{1} \frac{\partial}{\partial x_{1}}\right) Y\left(\tau,\left(x_{1}, \varphi_{1}\right)\right) .
\end{aligned}
$$

Thus

$$
\begin{gathered}
\varphi_{1}\left[\sum_{j \in \mathbb{Z}_{+}}\left(t^{j} B_{j}^{(0)} L(-j)+t^{j-\frac{1}{2}} N_{j-\frac{1}{2}}^{(0)} G\left(-j+\frac{1}{2}\right)\right), Y(u,(x, \varphi))\right] \\
=\varphi_{1} \operatorname{Res}_{x_{1}}\left[\left(\frac{1}{2} l_{t}\left(x_{1}\right)\left(\frac{\partial}{\partial \varphi_{1}}+\varphi_{1} \frac{\partial}{\partial x_{1}}\right)+g_{t}\left(x_{1}\right)\right) Y\left(\tau,\left(x_{1}, \varphi_{1}\right)\right), Y(u,(x, \varphi))\right] \\
=\varphi_{1} \operatorname{Res}_{x_{1}}\left(\left(\frac{1}{2} l_{t}\left(x_{1}\right)\left(\frac{\partial}{\partial \varphi_{1}}+\varphi_{1} \frac{\partial}{\partial x_{1}}\right)+g_{t}\left(x_{1}\right)\right)\right. \\
\left.\operatorname{Res}_{x_{0}} x^{-1} \delta\left(\frac{x_{1}-x_{0}-\varphi_{1} \varphi}{x}\right) Y\left(Y\left(\tau,\left(x_{0}, \varphi_{1}-\varphi\right)\right) u,(x, \varphi)\right)\right) \\
=Y\left(\varphi_{1} \operatorname{Res}_{x_{1}} \operatorname{Res}_{x_{0}}\left(\frac{1}{2} l_{t}\left(x_{1}\right)\left(\frac{\partial}{\partial \varphi_{1}}+\varphi_{1} \frac{\partial}{\partial x_{1}}\right)+g_{t}\left(x_{1}\right)\right)\right. \\
\left.x^{-1} \delta\left(\frac{x_{1}-x_{0}-\varphi_{1} \varphi}{x}\right) Y\left(\tau,\left(x_{0}, \varphi_{1}-\varphi\right)\right) u,(x, \varphi)\right) .
\end{gathered}
$$

But

$$
\begin{gathered}
\varphi_{1} \operatorname{Res}_{x_{1}} \operatorname{Res}_{x_{0}}\left(\frac{1}{2} l_{t}\left(x_{1}\right)\left(\frac{\partial}{\partial \varphi_{1}}+\varphi_{1} \frac{\partial}{\partial x_{1}}\right)+g_{t}\left(x_{1}\right)\right) \\
x^{-1} \delta\left(\frac{x_{1}-x_{0}-\varphi_{1} \varphi}{x}\right) Y\left(\tau,\left(x_{0}, \varphi_{1}-\varphi\right)\right) \\
=\varphi_{1} \operatorname{Res}_{x_{1}} \operatorname{Res}_{x_{0}}\left(\frac{1}{2} l_{t}\left(x_{1}\right)\left(\frac{\partial}{\partial \varphi_{1}}+\varphi_{1} \frac{\partial}{\partial x_{1}}\right)+g_{t}\left(x_{1}\right)\right) \\
x_{1}^{-1} \delta\left(\frac{x+x_{0}+\varphi_{1} \varphi}{x_{1}}\right) Y\left(\tau,\left(x_{0}, \varphi_{1}-\varphi\right)\right) \\
=\varphi_{1} \operatorname{Res}_{x_{1}} \operatorname{Res}_{x_{0}}\left(\frac{1}{2} l_{t}\left(x_{1}\right)\left(\frac{\partial}{\partial \varphi_{1}} x_{1}^{-1} \delta\left(\frac{x+x_{0}+\varphi_{1} \varphi}{x_{1}}\right)\right) Y\left(\tau,\left(x_{0},-\varphi\right)\right)\right. \\
+\frac{1}{2} l_{t}\left(x_{1}\right) x_{1}^{-1} \delta\left(\frac{x+x_{0}}{x_{1}}\right) \frac{\partial}{\partial \varphi_{1}} Y\left(\tau,\left(x_{0}, \varphi_{1}\right)\right) \\
\left.+g_{t}\left(x_{1}\right) x_{1}^{-1} \delta\left(\frac{x+x_{0}}{x_{1}}\right) Y\left(\tau,\left(x_{0},-\varphi\right)\right)\right)
\end{gathered}
$$




$$
\begin{aligned}
& =\varphi_{1} \operatorname{Res}_{x_{1}} \operatorname{Res}_{x_{0}}\left(\left(\sum_{n \in \mathbb{Z}} n\left(x+x_{0}\right)^{n-1} \varphi x_{1}^{-n-1}\right)\left(\frac{1}{2} \sum_{j \in \mathbb{Z}_{+}} t^{j} B_{j}^{(0)} x_{1}^{-j+1}\right)\right. \\
& \left(\sum_{k \in \mathbb{Z}} G\left(k+\frac{1}{2}\right) x_{0}^{-k-2}\right)+\left(\sum_{n \in \mathbb{Z}}\left(x+x_{0}\right)^{n} x_{1}^{-n-1}\right)\left(\frac{1}{2} \sum_{j \in \mathbb{Z}_{+}} t^{j} B_{j}^{(0)} x_{1}^{-j+1}\right) \\
& \left(2 \sum_{k \in \mathbb{Z}} L(k) x_{0}^{-k-2}\right)+\left(\sum_{n \in \mathbb{Z}}\left(x+x_{0}\right)^{n} x_{1}^{-n-1}\right)\left(\sum_{j \in \mathbb{Z}_{+}} t^{j-\frac{1}{2}} N_{j-\frac{1}{2}}^{(0)} x_{1}^{-j+1}\right) \\
& \left.\left(\sum_{k \in \mathbb{Z}} G\left(k+\frac{1}{2}\right) x_{0}^{-k-2}-2 \varphi L(k) x_{0}^{-k-2}\right)\right) \\
& =\varphi_{1} \operatorname{Res}_{x_{0}}\left(\sum _ { l \in \mathbb { N } } \sum _ { k \in \mathbb { Z } } \sum _ { j \in \mathbb { Z } _ { + } } \left((-j+1)\left(\begin{array}{c}
-j \\
l
\end{array}\right) x^{-j-l} x_{0}^{l} \frac{\varphi}{2} t^{j} B_{j}^{(0)} G\left(k+\frac{1}{2}\right) x_{0}^{-k-2}\right.\right. \\
& +\left(\begin{array}{c}
-j+1 \\
l
\end{array}\right) x^{-j-l+1} x_{0}^{l} t^{j} B_{j}^{(0)} L(k) x_{0}^{-k-2} \\
& \left.\left.+\left(\begin{array}{c}
-j+1 \\
l
\end{array}\right) x^{-j-l+1} x_{0}^{l} t^{j-\frac{1}{2}} N_{j-\frac{1}{2}}^{(0)}\left(G\left(k+\frac{1}{2}\right)-2 \varphi L(k)\right) x_{0}^{-k-2}\right)\right) \\
& =\varphi_{1} \sum_{l \in \mathbb{N}} \sum_{j \in \mathbb{Z}_{+}}\left(\frac{(-j+1)}{2}\left(\begin{array}{c}
-j \\
l
\end{array}\right) x^{-j-l} \varphi t^{j} B_{j}^{(0)} G\left(l-\frac{1}{2}\right)\right. \\
& +\left(\begin{array}{c}
-j+1 \\
l
\end{array}\right) x^{-j-l+1} t^{j} B_{j}^{(0)} L(l-1) \\
& \left.+\left(\begin{array}{c}
-j+1 \\
l
\end{array}\right) x^{-j-l+1} t^{j-\frac{1}{2}} N_{j-\frac{1}{2}}^{(0)}\left(G\left(l-\frac{1}{2}\right)-2 \varphi L(l-1)\right)\right) \\
& =\varphi_{1} \sum_{m=-1}^{\infty} \sum_{j \in \mathbb{Z}_{+}}\left(( \begin{array} { c } 
{ - j + 1 } \\
{ m + 1 }
\end{array} ) \left(t^{j} B_{j}^{(0)} L(m)+t^{j-\frac{1}{2}} N_{j-\frac{1}{2}}^{(0)}\left(G\left(m+\frac{1}{2}\right)\right) x^{-j-m}\right.\right. \\
& \left.+\varphi\left(\begin{array}{c}
-j+1 \\
m+1
\end{array}\right)\left(\frac{(-j-m)}{2} x^{-1} t^{j} B_{j}^{(0)} G\left(m+\frac{1}{2}\right)+2 t^{j-\frac{1}{2}} N_{j-\frac{1}{2}}^{(0)} L(m)\right) x^{-j-m}\right) \\
& =\varphi_{1} \sum_{m=-1}^{\infty} \sum_{j \in \mathbb{Z}_{+}}\left(\begin{array}{c}
-j+1 \\
m+1
\end{array}\right) x^{-j-m}\left(\left(t^{j} B_{j}^{(0)}+2 \varphi t^{j-\frac{1}{2}} N_{j-\frac{1}{2}}^{(0)}\right) L(m)\right. \\
& \left.+\left(t^{j-\frac{1}{2}} N_{j-\frac{1}{2}}^{(0)}+\varphi x^{-1} \frac{(-j-m)}{2} t^{j} B_{j}^{(0)}\right) G\left(m+\frac{1}{2}\right)\right) .
\end{aligned}
$$

This finishes the proof of (7.14) thus concluding the proof of the sewing axiom for case (b).

(c) We prove the sewing axiom for the special case $Q_{12} \infty_{0} Q_{2}$ when

$$
Q_{1}=\left((z, \theta) ; \mathbf{0},\left(a^{(1)}, A^{(1)}, M^{(1)}\right),\left(a^{(2)}, A^{(2)}, M^{(2)}\right)\right) \in S K(2)
$$

and

$$
Q_{2}=\left(\left(B^{(0)}, N^{(0)}\right),\left(b^{(1)}, B^{(1)}, N^{(1)}\right)\right) \in S K(1) .
$$


Using steps (a) and (b) and Propositions 2.5 4.1 and 7.1 we have

$$
\begin{aligned}
& \left(\nu_{2}^{Y}\left(Q_{1}\right)_{2} *_{0} \nu_{1}^{Y}\left(Q_{2}\right)\right) e^{-\Gamma\left(a^{(2)}, A^{(2)}, M^{(2)}, B^{(0)}, N^{(0)}\right) c} \\
& \quad=\left(\nu_{2}^{Y}\left(\left((z, \theta) ; \mathbf{0},\left(a^{(1)}, A^{(1)}, M^{(1)}\right),(1, \mathbf{0})\right)_{2} \infty_{0}\left(\mathbf{0},\left(a^{(2)}, A^{(2)}, M^{(2)}\right)\right)\right)_{2} *_{0}\right. \\
& \left.\nu_{1}^{Y}\left(Q_{2}\right)\right) e^{-\Gamma\left(a^{(2)}, A^{(2)}, M^{(2)}, B^{(0)}, N^{(0)}\right) c} \\
& =\left(\left(\nu_{2}^{Y}\left((z, \theta) ; \mathbf{0},\left(a^{(1)}, A^{(1)}, M^{(1)}\right),(1, \mathbf{0})\right)_{2} *_{0} \nu_{1}^{Y}\left(\mathbf{0},\left(a^{(2)}, A^{(2)}, M^{(2)}\right)\right)\right)_{2} *_{0}\right. \\
& \left.\nu_{1}^{Y}\left(Q_{2}\right)\right) e^{-\Gamma\left(a^{(2)}, A^{(2)}, M^{(2)}, B^{(0)}, N^{(0)}\right) c} \\
& =\left(\nu _ { 2 } ^ { Y } ( ( z , \theta ) ; \mathbf { 0 } , ( a ^ { ( 1 ) } , A ^ { ( 1 ) } , M ^ { ( 1 ) } ) , ( 1 , \mathbf { 0 } ) ) _ { 2 } * _ { 0 } \left(\nu_{1}^{Y}\left(\mathbf{0},\left(a^{(2)}, A^{(2)}, M^{(2)}\right)\right)_{1} *_{0}\right.\right. \\
& \left.\left.\quad \nu_{1}^{Y}\left(Q_{2}\right)\right)\right) e^{-\Gamma\left(a^{(2)}, A^{(2)}, M^{(2)}, B^{(0)}, N^{(0)}\right) c} \\
& = \\
& =\nu_{2}^{Y}\left((z, \theta) ; \mathbf{0},\left(a^{(1)}, A^{(1)}, M^{(1)}\right),(1, \mathbf{0})\right)_{2} *_{0} \nu_{1}^{Y}\left(\left(\mathbf{0},\left(a^{(2)}, A^{(2)}, M^{(2)}\right)\right)_{1} \infty_{0} Q_{2}\right) \\
& =\nu_{2}^{Y}\left(\left((z, \theta) ; \mathbf{0},\left(a^{(1)}, A^{(1)}, M^{(1)}\right),(1, \mathbf{0})\right)_{2} \infty_{0}\left(\left(\mathbf{0},\left(a^{(2)}, A^{(2)}, M^{(2)}\right)\right)_{1} \infty_{0} Q_{2}\right)\right) \\
& \left.=\nu_{2}^{Y}\left(\left((z, \theta) ; \mathbf{0},\left(a^{(1)}, A^{(1)}, M_{0} Q_{2}\right),(1, \mathbf{0})\right)_{2} \infty_{0}\left(\mathbf{0},\left(a^{(2)}, A^{(2)}, M^{(2)}\right)\right)\right)_{2} \infty_{0} Q_{2}\right)
\end{aligned}
$$

(d) We prove the sewing axiom for the special case $Q_{1}{ }_{1} \infty_{0} Q_{2}$ when

$$
Q_{1}=((z, \theta) ; \mathbf{0},(1, \mathbf{0}),(1, \mathbf{0})) \in S K(2)
$$

and

$$
Q_{2}\left(\left(B^{(0)}, N^{(0)}\right),\left(b^{(1)}, B^{(1)}, N^{(1)}\right)\right) \in S K(1) .
$$

In this case,

$$
\begin{aligned}
& \left\langle v^{\prime},\left(\nu_{2}^{Y}\left(Q_{1}\right){ }_{1} *_{0} \nu_{1}^{Y}\left(Q_{2}\right)\right)_{t^{1 / 2}}\left(v_{1} \otimes v_{2}\right)\right\rangle \\
& =\sum_{k \in \frac{1}{2} \mathbb{Z}} \sum_{i^{(k)}=1}^{\operatorname{dim} V_{(k)}}\left\langle v^{\prime}, Y\left(e_{i^{(k)}}^{(k)},(x, \varphi)\right) v_{2}\right\rangle \cdot\left\langle e^{-\sum_{j \in \mathbb{Z}_{+}}\left(B_{j}^{(0)} L^{\prime}(j)+N_{j-\frac{1}{2}}^{(0)} G^{\prime}\left(j-\frac{1}{2}\right)\right)} .\right. \\
& \left.\quad\left(e_{i(k)}^{(k)}\right)^{*}, e^{-\sum_{j \in \mathbb{Z}_{+}}\left(B_{j}^{(1)} L(j)+N_{j-\frac{1}{2}}^{(1)} G\left(j-\frac{1}{2}\right)\right)} \cdot\left(b^{(1)}\right)^{-2 L(0)} \cdot v_{1}\right\rangle\left. t^{k}\right|_{(x, \varphi)=(z, \theta)} \\
& =\sum_{k \in \frac{1}{2} \mathbb{Z}} \sum_{i^{(k)}=1}^{\operatorname{dim} V_{(k)}}\left\langle v^{\prime}, Y\left(e_{i^{(k)}}^{(k)},(x, \varphi)\right) v_{2}\right\rangle \cdot\left\langle\left(e_{i^{(k)}}^{(k)}\right)^{*},\right. \\
& e^{-\sum_{j \in \mathbb{Z}_{+}}\left(t^{j} B_{j}^{(0)} L(-j)+t^{j-\frac{1}{2}} N_{j-\frac{1}{2}}^{(0)} G\left(-j+\frac{1}{2}\right)\right)} t^{L(0)} \cdot \\
& \left.\quad e^{-\sum_{j \in \mathbb{Z}_{+}}\left(B_{j}^{(1)} L(j)+N_{j-\frac{1}{2}}^{(1)} G\left(j-\frac{1}{2}\right)\right)} \cdot\left(b^{(1)}\right)^{-2 L(0)} \cdot v_{1}\right\rangle\left.\right|_{(x, \varphi)=(z, \theta)}, e^{-\sum_{j \in \mathbb{Z}_{+}}\left(t^{j} B_{j}^{(0)} L(-j)+t^{j-\frac{1}{2}} N_{j-\frac{1}{2}}^{(0)} G\left(-j+\frac{1}{2}\right)\right)} t^{L(0)} \cdot \\
& \left.\left.e^{-\sum_{j \in \mathbb{Z}_{+}}\left(B_{j}^{(1)} L(j)+N_{j-\frac{1}{2}}^{(1)} G\left(j-\frac{1}{2}\right)\right)} \cdot\left(b^{(1)}\right)^{-2 L(0)} \cdot v_{1},(x, \varphi)\right) \cdot v_{2}\right\rangle\left.\right|_{(x, \varphi)=(z, \theta)} \\
& \quad e^{-\sum_{j \in \mathbb{Z}_{+}}\left(t^{j} B_{j}^{(0)} L(-j)+t^{j-\frac{1}{2}} N_{j-\frac{1}{2}}^{(0)} G\left(-j+\frac{1}{2}\right)\right)} t^{L(0)} \cdot \\
& \left.e^{-\sum_{j \in \mathbb{Z}_{+}}\left(B_{j}^{(1)} L(j)+N_{j-\frac{1}{2}}^{(1)} G\left(j-\frac{1}{2}\right)\right)} \cdot\left(b^{(1)}\right)^{-2 L(0)} \cdot v_{1}\right\rangle\left.\right|_{(x, \varphi)=(z, \theta)}
\end{aligned}
$$




$$
\begin{gathered}
=(-1)^{\eta\left(v_{1}\right) \eta\left(v_{2}\right)}\left\langle e^{x L^{\prime}(1)+\varphi G^{\prime}\left(\frac{1}{2}\right)} v^{\prime}, Y\left(v_{2},(-x,-\varphi)\right)\right. \\
e^{-\sum_{j \in \mathbb{Z}_{+}}\left(t^{j} B_{j}^{(0)} L(-j)+t^{j-\frac{1}{2}} N_{j-\frac{1}{2}}^{(0)} G\left(-j+\frac{1}{2}\right)\right)} t^{L(0)} . \\
\left.\quad e^{-\sum_{j \in \mathbb{Z}_{+}}\left(B_{j}^{(1)} L(j)+N_{j-\frac{1}{2}}^{(1)} G\left(j-\frac{1}{2}\right)\right)} \cdot\left(b^{(1)}\right)^{-2 L(0)} \cdot v_{1}\right\rangle\left.\right|_{(x, \varphi)=(z, \theta)} .
\end{gathered}
$$

By (7.9) in case (b), the right-hand side of (7.15) is equal to

$$
\begin{aligned}
& (-1)^{\eta\left(v_{1}\right) \eta\left(v_{2}\right)}\left(\nu_{2}^{Y}((-z,-\theta) ; \mathbf{0},(1, \mathbf{0}),(1, \mathbf{0}))\right. \\
& \left.2 *_{0} \nu_{1}^{Y}\left(\left(B^{(0)}, N^{(0)}\right),\left(b^{(1)}, B^{(1)}, N^{(1)}\right)\right)\right)_{t^{1 / 2}}\left(e^{z L^{\prime}(1)+\theta G^{\prime}\left(\frac{1}{2}\right)} v^{\prime} \otimes v_{2} \otimes v_{1}\right) .
\end{aligned}
$$

Let $\Theta_{j}^{(2)}$, for $j \in \frac{1}{2} \mathbb{Z}_{+}$, be defined by (7.12), and let $H_{t^{1 / 2}}(x, \varphi)=\left(H_{t^{1 / 2}}^{0}(x, \varphi), H_{t^{1 / 2}}^{1}(x, \varphi)\right)$ be defined by (7.13). Then by case (b), we have

$$
\begin{aligned}
& \left\langle v^{\prime},\left(\nu_{2}^{Y}\left(Q_{1}\right)_{1} *_{0} \nu_{1}^{Y}\left(Q_{2}\right)\right)_{t^{1 / 2}}\left(v_{1} \otimes v_{2}\right)\right\rangle \\
& =(-1)^{\eta\left(v_{1}\right) \eta\left(v_{2}\right)}\left\langle e^{-\sum_{j \in \mathbb{Z}_{+}}\left(t^{j} B_{j}^{(0)} L^{\prime}(j)+t^{j-\frac{1}{2}} N_{j-\frac{1}{2}}^{(0)} G^{\prime}\left(j-\frac{1}{2}\right)\right)} e^{-x L^{\prime}(1)-\varphi G^{\prime}\left(\frac{1}{2}\right)} v^{\prime},\right. \\
& Y\left(e^{-\sum_{j \in \mathbb{Z}_{+}}\left(\Theta_{j}^{(2)} L(j)+\Theta_{j-\frac{1}{2}}^{(2)} G\left(j-\frac{1}{2}\right)\right)} e^{-2 \Theta_{0}^{(2)} L(0)} v_{2}, H_{t^{1 / 2}}^{-1} \circ(x, \varphi)\right) \\
& \left.t^{L(0)} e^{-\sum_{j \in \mathbb{Z}_{+}}\left(B_{j}^{(1)} L(j)+N_{j-\frac{1}{2}}^{(1)} G\left(j-\frac{1}{2}\right)\right)}\left(b_{0}^{(1)}\right)^{-L(0)} \cdot v_{1}\right\rangle\left.\right|_{(x, \varphi)=(-z,-\theta)} \\
& =\left\langle e^{\left(H_{t^{1 / 2}}^{0}(x, \varphi) L^{\prime}(1)+H_{t^{1 / 2}}^{1}(x, \varphi) G^{\prime}\left(\frac{1}{2}\right)\right)} e^{-\sum_{j \in \mathbb{Z}_{+}}\left(t^{j} B_{j}^{(0)} L^{\prime}(j)+t^{j-\frac{1}{2}} N_{j-\frac{1}{2}}^{(0)} G^{\prime}\left(j-\frac{1}{2}\right)\right)}\right. \\
& e^{-x L^{\prime}(1)-\varphi G^{\prime}\left(\frac{1}{2}\right)} v^{\prime}, Y\left(t^{L(0)} e^{-\sum_{j \in \mathbb{Z}_{+}}\left(B_{j}^{(1)} L(j)+N_{j-\frac{1}{2}}^{(1)} G\left(j-\frac{1}{2}\right)\right)}\left(b^{(1)}\right)^{-2 L(0)} \cdot v_{1},\right. \\
& \left.\left.-H_{t^{1 / 2}}^{-1} \circ I(x, \varphi)\right) e^{-\sum_{j \in \mathbb{Z}_{+}}\left(\Theta_{j}^{(2)} L(j)+\Theta_{j-\frac{1}{2}}^{(2)} G\left(j-\frac{1}{2}\right)\right)} e^{-2 \Theta_{0}^{(2)} L(0)} v_{2}\right\rangle\left.\right|_{(x, \varphi)=(-z,-\theta)} .
\end{aligned}
$$

The right-hand side of the above expression converges when $t^{1 / 2}=1$ and, by the definition of sewing, is equal to

$$
\left\langle v^{\prime}, \nu_{2}^{Y}\left(Q_{1}{ }_{1} \infty_{0} Q_{2}\right)\left(v_{1} \otimes v_{2}\right)\right\rangle
$$

(e) We prove the sewing axiom for the special case $Q_{1}{ }_{1} \infty_{0} Q_{2}$ when

$$
Q_{1}=\left((z, \theta) ; \mathbf{0},\left(a^{(1)}, A^{(1)}, M^{(1)}\right),\left(a^{(2)}, A^{(2)}, M^{(2)}\right)\right) \in S K(2)
$$

and

$$
Q_{2}\left(\left(B^{(0)}, N^{(0)}\right),\left(b^{(1)}, B^{(1)}, N^{(1)}\right)\right) \in S K(1) .
$$

Using cases (a), (c) and (d) and Propositions 2.5 4.1 and 7.1 and letting

$$
e^{-\Gamma\left(a^{(1)}, A^{(1)}, M^{(1)}, B^{(0)}, N^{(0)}\right) c}=e^{-\Gamma c},
$$


we have

$$
\begin{array}{cc}
\left(\nu_{2}^{Y}\left(Q_{1}\right)_{1} *_{0} \nu_{1}^{Y}\left(Q_{2}\right) e^{-\Gamma\left(a^{(1)}, A^{(1)}, M^{(1)}, B^{(0)}, N^{(0)}\right) c}\right. \\
=\quad\left(\nu _ { 2 } ^ { Y } \left(\left(((z, \theta) ; \mathbf{0},(1, \mathbf{0}),(1, \mathbf{0}))_{1} \infty_{0}\left(\mathbf{0},\left(a^{(1)}, A^{(1)}, M^{(1)}\right)\right)\right)_{2} \infty_{0}\right.\right. \\
\left.\left.\quad\left(\mathbf{0},\left(a^{(2)}, A^{(2)}, M^{(2)}\right)\right)\right)_{1} *_{0} \nu_{1}^{Y}\left(\left(B^{(0)}, N^{(0)}\right),\left(b^{(1)}, B^{(1)}, N^{(1)}\right)\right)\right) e^{-\Gamma c} \\
=\quad\left(\left(\left(\nu_{2}^{Y}((z, \theta) ; \mathbf{0},(1, \mathbf{0}),(1, \mathbf{0}))_{1} *_{0} \nu_{1}^{Y}\left(\mathbf{0},\left(a^{(1)}, A^{(1)}, M^{(1)}\right)\right)\right)_{2} *_{0}\right.\right. \\
\left.\left.\quad \nu_{1}^{Y}\left(\mathbf{0},\left(a^{(2)}, A^{(2)}, M^{(2)}\right)\right)\right)_{1} *_{0} \nu_{1}^{Y}\left(\left(B^{(0)}, N^{(0)}\right),\left(b^{(1)}, B^{(1)}, N^{(1)}\right)\right)\right) e^{-\Gamma c} \\
=\quad\left(\left(\nu _ { 2 } ^ { Y } ( ( z , \theta ) ; \mathbf { 0 } , ( 1 , \mathbf { 0 } ) , ( 1 , \mathbf { 0 } ) ) _ { 1 } * _ { 0 } \left(\nu_{1}^{Y}\left(\mathbf{0},\left(a^{(1)}, A^{(1)}, M^{(1)}\right)\right)_{1} *_{0}\right.\right.\right. \\
\left.\left.\left.\quad \nu_{1}^{Y}\left(\left(B^{(0)}, N^{(0)}\right),\left(b^{(1)}, B^{(1)}, N^{(1)}\right)\right)\right)\right)_{2} *_{0} \nu_{1}^{Y}\left(\mathbf{0},\left(a^{(2)}, A^{(2)}, M^{(2)}\right)\right)\right) e^{-\Gamma c} \\
=\quad\left(\nu _ { 2 } ^ { Y } ( ( z , \theta ) ; \mathbf { 0 } , ( 1 , \mathbf { 0 } ) , ( 1 , \mathbf { 0 } ) ) _ { 1 } * _ { 0 } \nu _ { 1 } ^ { Y } \left(\left(\mathbf{0},\left(a^{(1)}, A^{(1)}, M^{(1)}\right)\right)_{1} \infty_{0}\right.\right. \\
\left.\left.\quad\left(\left(B^{(0)}, N^{(0)}\right),\left(b^{(1)}, B^{(1)}, N^{(1)}\right)\right)\right)\right)_{2} *_{0} \nu_{1}^{Y}\left(\mathbf{0},\left(a^{(2)}, A^{(2)}, M^{(2)}\right)\right) \\
=\quad \nu_{2}^{Y}\left(( ( z , \theta ) ; \mathbf { 0 } , ( 1 , \mathbf { 0 } ) , ( 1 , \mathbf { 0 } ) ) _ { 1 } \infty _ { 0 } \left(\left(\mathbf{0},\left(a^{(1)}, A^{(1)}, M^{(1)}\right)\right){ }_{1} \infty_{0}\right.\right. \\
\left.\left.\quad\left(\left(B^{(0)}, N^{(0)}\right),\left(b^{(1)}, B^{(1)}, N^{(1)}\right)\right)\right)\right)_{2} *_{0} \nu_{1}^{Y}\left(\mathbf{0},\left(a^{(2)}, A^{(2)}, M^{(2)}\right)\right) \\
=\quad \nu_{2}^{Y}\left(\left(( ( z , \theta ) ; \mathbf { 0 } , ( 1 , \mathbf { 0 } ) , ( 1 , \mathbf { 0 } ) ) _ { 1 } \infty _ { 0 } \left(\left(\mathbf{0},\left(a^{(1)}, A^{(1)}, M^{(1)}\right)\right)_{1} \infty_{0}\right.\right.\right. \\
& \left.\left.\left.\left(\left(B^{(0)}, N^{(0)}\right),\left(b^{(1)}, B^{(1)}, N^{(1)}\right)\right)\right)\right)_{2} \infty_{0}\left(\mathbf{0},\left(a^{(2)}, A^{(2)}, M^{(2)}\right)\right)\right) \\
= & \nu_{2}^{Y}\left(\left(\left(((z, \theta) ; \mathbf{0},(1, \mathbf{0}),(1, \mathbf{0}))_{1} \infty_{0}\left(\mathbf{0},\left(a^{(1)}, A^{(1)}, M^{(1)}\right)\right)\right)_{2} \infty_{0}\right.\right. \\
& \left.\left.\left(\mathbf{0},\left(a^{(2)}, A^{(2)}, M^{(2)}\right)\right)\right){ }_{1} \infty_{0}\left(\left(B^{(0)}, N^{(0)}\right),\left(b^{(1)}, B^{(1)}, N^{(1)}\right)\right)\right) \\
= & \nu_{2}^{Y}\left(Q_{1} \infty_{0} Q_{2}\right)
\end{array}
$$

(f) We prove the sewing axiom for the special case $Q_{1}{ }_{m} \infty_{0} Q_{2}$ when

$$
\begin{aligned}
Q_{1}=\left(\left(z_{1}, \theta_{1}\right), \ldots,\left(z_{m-1}, \theta_{m-1}\right) ;\left(A^{(0)}, M^{(0)}\right),\left(a^{(1)}, A^{(1)}, M^{(1)}\right), \ldots,\right. & \\
& \left.\left(a^{(m)}, A^{(m)}, M^{(m)}\right)\right) \in S K(m),
\end{aligned}
$$

and

$$
Q_{2}=\left((z, \theta) ; \mathbf{0},\left(b^{(1)}, B^{(1)}, N^{(1)}\right),\left(b^{(2)}, B^{(2)}, N^{(2)}\right)\right) \in S K(2) .
$$

In this case,

$$
\begin{aligned}
& \left\langle v^{\prime},\left(\nu_{m}^{Y}\left(Q_{1}\right) m_{m} *_{0} \nu_{2}^{Y}\left(Q_{1}\right)\right)_{t^{1 / 2}}\left(v_{1} \otimes \cdots v_{m+1}\right)\right\rangle \\
& =\sum_{k \in \frac{1}{2} \mathbb{Z}} \sum_{i(k)=1}^{\operatorname{dim} V_{(k)}} \iota_{1 \cdots m-1}^{-1}\left\langle e^{-\sum_{j \in \mathbb{Z}_{+}}\left(A_{j}^{(0)} L^{\prime}(j)+M_{j-\frac{1}{2}}^{(0)} G^{\prime}\left(j-\frac{1}{2}\right)\right)} v^{\prime},\right. \\
& Y\left(e^{-\sum_{j \in \mathbb{Z}_{+}}\left(A_{j}^{(1)} L(j)+M_{j-\frac{1}{2}}^{(1)} G\left(j-\frac{1}{2}\right)\right)} \cdot\left(a^{(1)}\right)^{-2 L(0)} \cdot v_{1},\left(x_{1}, \varphi_{1}\right)\right) \cdots \\
& Y\left(e^{-\sum_{j \in \mathbb{Z}_{+}}\left(A_{j}^{(m-1)} L(j)+M_{j-\frac{1}{2}}^{(m-1)} G\left(j-\frac{1}{2}\right)\right)} \cdot\left(a^{(m-1)}\right)^{-2 L(0)} \cdot v_{m-1},\right. \\
& \left.\left.\left(x_{m-1}, \varphi_{m-1}\right)\right) \cdot e^{-\sum_{j \in \mathbb{Z}_{+}}\left(A_{j}^{(m)} L(j)+M_{j-\frac{1}{2}}^{(m)} G\left(j-\frac{1}{2}\right)\right)} \cdot\left(a^{(m)}\right)^{-2 L(0)} \cdot e_{i(k)}^{(k)}\right\rangle . \\
& \left\langle\left(e_{i(k)}^{(k)}\right)^{*}, Y\left(e^{-\sum_{j \in \mathbb{Z}_{+}}\left(B_{j}^{(1)} L(j)+N_{j-\frac{1}{2}}^{(1)} G\left(j-\frac{1}{2}\right)\right)} \cdot\left(b^{(1)}\right)^{-2 L(0)} \cdot v_{m},(x, \varphi)\right) .\right. \\
& \left.e^{-\sum_{j \in \mathbb{Z}_{+}}\left(B_{j}^{(2)} L(j)+N_{j-\frac{1}{2}}^{(2)} G\left(j-\frac{1}{2}\right)\right)} \cdot\left(b^{(2)}\right)^{-2 L(0)} \cdot v_{m+1}\right\rangle t^{k} \mid \begin{array}{c}
\left(x_{i}, \varphi_{i}\right)=\left(z_{i}, \theta_{i}\right), \\
(x, \varphi)=(z, \theta)
\end{array}
\end{aligned}
$$




$$
\begin{aligned}
& =\sum_{k \in \frac{1}{2} \mathbb{Z}} \sum_{i^{(k)}=1}^{\operatorname{dim} V_{(k)}} \iota_{1 \cdots m-1}^{-1}\left\langle e^{-\sum_{j \in \mathbb{Z}_{+}}\left(A_{j}^{(0)} L^{\prime}(j)+M_{j-\frac{1}{2}}^{(0)} G^{\prime}\left(j-\frac{1}{2}\right)\right)} v^{\prime},\right. \\
& Y\left(e^{-\sum_{j \in \mathbb{Z}_{+}}\left(A_{j}^{(1)} L(j)+M_{j-\frac{1}{2}}^{(1)} G\left(j-\frac{1}{2}\right)\right)} \cdot\left(a^{(1)}\right)^{-2 L(0)} \cdot v_{1},\left(x_{1}, \varphi_{1}\right)\right) \cdots \\
& Y\left(e^{-\sum_{j \in \mathbb{Z}_{+}}\left(A_{j}^{(m-1)} L(j)+M_{j-\frac{1}{2}}^{(m-1)} G\left(j-\frac{1}{2}\right)\right)} \cdot\left(a^{(m-1)}\right)^{-2 L(0)} \cdot v_{m-1},\right. \\
& \left.\left.\left(x_{m-1}, \varphi_{m-1}\right)\right) \cdot e^{-\sum_{j \in \mathbb{Z}_{+}}\left(A_{j}^{(m)} L(j)+M_{j-\frac{1}{2}}^{(m)} G\left(j-\frac{1}{2}\right)\right)} \cdot\left(a^{(m)}\right)^{-2 L(0)} \cdot e_{i^{(k)}}^{(k)}\right\rangle . \\
& \left\langle t^{L^{\prime}(0)}\left(e_{i(k)}^{(k)}\right)^{*}, Y\left(e^{-\sum_{j \in \mathbb{Z}_{+}}\left(B_{j}^{(1)} L(j)+N_{j-\frac{1}{2}}^{(1)} G\left(j-\frac{1}{2}\right)\right)} \cdot\left(b^{(1)}\right)^{-2 L(0)} \cdot v_{m},\right.\right. \\
& (x, \varphi)) \cdot e^{-\sum_{j \in \mathbb{Z}_{+}}\left(B_{j}^{(2)} L(j)+N_{j-\frac{1}{2}}^{(2)} G\left(j-\frac{1}{2}\right)\right)} . \\
& \left.\left(b^{(2)}\right)^{-2 L(0)} \cdot v_{m+1}\right\rangle \mid \begin{array}{c}
\left(x_{i}, \varphi_{i}\right)=\left(z_{i}, \theta_{i}\right) \\
(x, \varphi)=(z, \theta)
\end{array} \\
& =\sum_{k \in \frac{1}{2} \mathbb{Z}} \sum_{i(k)=1}^{\operatorname{dim} V_{(k)}} \iota_{1 \cdots m-1}^{-1}\left\langle e^{-\sum_{j \in \mathbb{Z}_{+}}\left(A_{j}^{(0)} L^{\prime}(j)+M_{j-\frac{1}{2}}^{(0)} G^{\prime}\left(j-\frac{1}{2}\right)\right)} v^{\prime},\right. \\
& Y\left(e^{-\sum_{j \in \mathbb{Z}_{+}}\left(A_{j}^{(1)} L(j)+M_{j-\frac{1}{2}}^{(1)} G\left(j-\frac{1}{2}\right)\right)} \cdot\left(a^{(1)}\right)^{-2 L(0)} \cdot v_{1},\left(x_{1}, \varphi_{1}\right)\right) \cdots \\
& Y\left(e^{-\sum_{j \in \mathbb{Z}_{+}}\left(A_{j}^{(m-1)} L(j)+M_{j-\frac{1}{2}}^{(m-1)} G\left(j-\frac{1}{2}\right)\right)} \cdot\left(a^{(m-1)}\right)^{-2 L(0)} \cdot v_{m-1},\right. \\
& \left.\left.\left(x_{m-1}, \varphi_{m-1}\right)\right) \cdot e^{-\sum_{j \in \mathbb{Z}_{+}}\left(A_{j}^{(m)} L(j)+M_{j-\frac{1}{2}}^{(m)} G\left(j-\frac{1}{2}\right)\right)} \cdot\left(a^{(m)}\right)^{-2 L(0)} \cdot e_{i^{(k)}}^{(k)}\right\rangle \cdot \\
& \left\langle\left(e_{i^{(k)}}^{(k)}\right)^{*}, t^{L(0)} Y\left(e^{-\sum_{j \in \mathbb{Z}_{+}}\left(B_{j}^{(1)} L(j)+N_{j-\frac{1}{2}}^{(1)} G\left(j-\frac{1}{2}\right)\right)} \cdot\left(b^{(1)}\right)^{-2 L(0)} \cdot v_{m},\right.\right. \\
& (x, \varphi)) \cdot e^{-\sum_{j \in \mathbb{Z}_{+}}\left(B_{j}^{(2)} L(j)+N_{j-\frac{1}{2}}^{(2)} G\left(j-\frac{1}{2}\right)\right)} . \\
& \left.\left(b^{(2)}\right)^{-2 L(0)} \cdot v_{m+1}\right\rangle \mid \begin{array}{c}
\left(x_{i}, \varphi_{i}\right)=\left(z_{i}, \theta_{i}\right) \\
(x, \varphi)=(z, \theta)
\end{array} \\
& (7.16)=\iota_{1 \cdots m}^{-1}\left\langle e^{-\sum_{j \in \mathbb{Z}_{+}}\left(A_{j}^{(0)} L^{\prime}(j)+M_{j-\frac{1}{2}}^{(0)} G^{\prime}\left(j-\frac{1}{2}\right)\right)} v^{\prime},\right. \\
& Y\left(e^{-\sum_{j \in \mathbb{Z}_{+}}\left(A_{j}^{(1)} L(j)+M_{j-\frac{1}{2}}^{(1)} G\left(j-\frac{1}{2}\right)\right)} \cdot\left(a^{(1)}\right)^{-2 L(0)} \cdot v_{1},\left(x_{1}, \varphi_{1}\right)\right) \cdots \\
& Y\left(e^{-\sum_{j \in \mathbb{Z}_{+}}\left(A_{j}^{(m-1)} L(j)+M_{j-\frac{1}{2}}^{(m-1)} G\left(j-\frac{1}{2}\right)\right)} \cdot\left(a^{(m-1)}\right)^{-2 L(0)} \cdot v_{m-1},\right. \\
& \left.\left(x_{m-1}, \varphi_{m-1}\right)\right) \cdot e^{-\sum_{j \in \mathbb{Z}_{+}}\left(A_{j}^{(m)} L(j)+M_{j-\frac{1}{2}}^{(m)} G\left(j-\frac{1}{2}\right)\right)} \cdot\left(t^{-\frac{1}{2}} a^{(m)}\right)^{-2 L(0)} . \\
& Y\left(e^{-\sum_{j \in \mathbb{Z}_{+}}\left(B_{j}^{(1)} L(j)+N_{j-\frac{1}{2}}^{(1)} G\left(j-\frac{1}{2}\right)\right)} \cdot\left(b^{(1)}\right)^{-2 L(0)} \cdot v_{m},(x, \varphi)\right) . \\
& \left.e^{-\sum_{j \in \mathbb{Z}_{+}}\left(B_{j}^{(2)} L(j)+N_{j-\frac{1}{2}}^{(2)} G\left(j-\frac{1}{2}\right)\right)} \cdot\left(b^{(2)}\right)^{-2 L(0)} \cdot v_{m+1}\right\rangle \mid \begin{array}{c}
\left(x_{i}, \varphi_{i}\right)=\left(z_{i}, \theta_{i}\right), \\
(x, \varphi)=(z, \theta)
\end{array} .
\end{aligned}
$$


Since $V$ is a positive energy representation for $\mathfrak{n s}$ and by the truncation condition (5.8) for $Y(\cdot,(x, \varphi))$, the right-hand side of (7.16) is a polynomial in $t^{1 / 2}$ and thus convergent when $t^{1 / 2}=1$. If we can prove

$$
\begin{array}{r}
e^{-\sum_{j \in \mathbb{Z}_{+}}\left(A_{j}^{(m)} L(j)+M_{j-\frac{1}{2}}^{(m)} G\left(j-\frac{1}{2}\right)\right)} \cdot\left(t^{-\frac{1}{2}} a^{(m)}\right)^{-2 L(0)} Y(u,(x, \varphi)) \cdot\left(t^{-\frac{1}{2}} a^{(m)}\right)^{2 L(0)} . \\
e^{\sum_{j \in \mathbb{Z}_{+}}\left(A_{j}^{(m)} L(j)+M_{j-\frac{1}{2}}^{(m)} G\left(j-\frac{1}{2}\right)\right)}
\end{array}
$$

$$
=Y\left(\left(t^{-\frac{1}{2}} a^{(m)}\right)^{-2 L(0)} e^{-\sum_{j \in \mathbb{Z}_{+}}\left(\Theta_{j}^{(1)} L(j)+\Theta_{j-\frac{1}{2}}^{(1)} G\left(j-\frac{1}{2}\right)\right)} \cdot e^{-2 \Theta_{0}^{(1)} L(0)} u, H_{t^{1 / 2}}^{-1}(x, \varphi)\right)
$$

where

$$
\begin{aligned}
& H_{t^{1 / 2}}^{-1}(x, \varphi) \\
& \quad=\left(t^{-\frac{1}{2}} a^{(m)}\right)^{2 L_{0}(x, \varphi)} \cdot \exp \left(\sum_{j \in \mathbb{Z}_{+}}\left(A_{j}^{(m)} L_{j}(x, \varphi)+M_{j-\frac{1}{2}}^{(m)} G_{j-\frac{1}{2}}(x, \varphi)\right)\right) \cdot(x, \varphi)
\end{aligned}
$$

and the $\Theta_{j}^{(1)}=\Theta_{j}^{(1)}\left(t^{-1 / 2} a_{\square}, A^{(m)}, M^{(m)},(x, \varphi)\right)$ are defined by 3.5), then since $e^{\Gamma\left(a^{(m)}, A^{(m)}, M^{(m)}, \mathbf{0}\right) c}=1$, when $t^{1 / 2}=1$, the right-hand side of (7.16) is equal to

$$
\left\langle v^{\prime}, \nu_{m+1}^{Y}\left(Q_{1} \infty_{0} Q_{2}\right)\left(v_{1} \otimes \cdots v_{m+1}\right)\right\rangle .
$$

Thus in this case, the proof of the sewing axiom is reduced to the proof of (7.17).

Let

$$
H_{t^{1 / 2}}^{-1}(x, \varphi)=\left(\tilde{x}\left(t^{\frac{1}{2}}\right), \tilde{\varphi}\left(t^{\frac{1}{2}}\right)\right) .
$$

Then by (5.15), (5.17) and Corollary 3.7 the right-hand side of (7.17) is equal to

$$
\begin{gathered}
\left(t^{-\frac{1}{2}} a^{(m)}\right)^{-2 L(0)} Y\left(e^{-\sum_{j \in \mathbb{Z}_{+}}\left(\Theta_{j}^{(1)} L(j)+\Theta_{j-\frac{1}{2}}^{(1)} G\left(j-\frac{1}{2}\right)\right)} \cdot e^{-2 \Theta_{0}^{(1)} L(0)} u,\right. \\
\left(\left(t^{-\frac{1}{2}} a^{(m)}\right)^{2} \tilde{x}\left(t^{\frac{1}{2}}\right),\left(t^{-\frac{1}{2}} a^{(m)}\right) \tilde{\varphi}\left(t^{\frac{1}{2}}\right)\right)\left(t^{-\frac{1}{2}} a^{(m)}\right)^{2 L(0)} \\
=\left(t^{-\frac{1}{2}} a^{(m)}\right)^{-2 L(0)} Y\left(e^{\left(\left(t^{-\frac{1}{2}} a^{(m)}\right)^{2} \tilde{x}\left(t^{\frac{1}{2}}\right)-x\right) L(-1)+\left(\left(t^{-\frac{1}{2}} a^{(m)}\right) \tilde{\varphi}\left(t^{\frac{1}{2}}\right)-\varphi\right) G\left(-\frac{1}{2}\right) .}\right. \\
\left.e^{-\sum_{j \in \mathbb{Z}_{+}}\left(\Theta_{j}^{(2)} L(j)+\Theta_{j-\frac{1}{2}}^{(2)} G\left(j-\frac{1}{2}\right)\right)} \cdot e^{-2 \Theta_{0}^{(2)} L(0)} u,(x, \varphi)\right)\left(t^{-\frac{1}{2}} a^{(m)}\right)^{2 L(0)} \\
=\left(t^{-\frac{1}{2}} a^{(m)}\right)^{-2 L(0)} Y\left(\operatorname { e x p } \left(-\sum_{k=-1}^{\infty} \sum_{j \in \mathbb{Z}_{+}}\left(\begin{array}{l}
j+1 \\
k+1
\end{array}\right) t^{j}\left(a^{(m)}\right)^{-2 j} x^{j-k}\right.\right. \\
\left(\left(A_{j}^{(m)}+2\left(\frac{j-k}{j+1}\right) t^{-\frac{1}{2}} a^{(m)} x^{-1} \varphi M_{j-\frac{1}{2}}^{(m)} L(k)\right.\right. \\
\left.\left.\left.+x^{-1}\left(\left(\frac{j-k}{j+1}\right) t^{-\frac{1}{2}} a^{(m)} M_{j-\frac{1}{2}}^{(m)}+\varphi \frac{(j-k)}{2} A_{j}^{(m)}\right) G\left(k+\frac{1}{2}\right)\right)\right) u,(x, \varphi)\right) . \\
\left(t^{-\frac{1}{2}} a^{(m)}\right)^{2 L(0)} .
\end{gathered}
$$


But since

$$
\begin{aligned}
& e^{-\sum_{j \in \mathbb{Z}_{+}}\left(A_{j} L(j)+A M j-\frac{1}{2} G\left(j-\frac{1}{2}\right)\right)}\left(t^{-\frac{1}{2}} a\right)^{-2 L(0)} \\
&=\left(t^{-\frac{1}{2}} a\right)^{-2 L(0)} e^{\sum_{j \in \mathbb{Z}_{+}}\left(t^{j} a^{-2 j} A_{j} L(j)+t^{j-\frac{1}{2}} a^{-2 j+1} M_{j-\frac{1}{2}} G\left(j-\frac{1}{2}\right)\right)},
\end{aligned}
$$

proving (7.17) is equivalent to proving

$$
\begin{aligned}
& e^{-\sum_{j \in \mathbb{Z}_{+}}\left(t^{j}\left(a^{(m)}\right)^{-2 j} A_{j}^{(m)} L(j)+t^{j-\frac{1}{2}}\left(a^{(m)}\right)^{-2 j+1} M_{j-\frac{1}{2}}^{(m)} G\left(j-\frac{1}{2}\right)\right)} Y(u,(x, \varphi)) . \\
& e^{\sum_{j \in \mathbb{Z}_{+}}\left(t^{j}\left(a^{(m)}\right)^{-2 j} A_{j}^{(m)} L(j)+t^{j-\frac{1}{2}}\left(a^{(m)}\right)^{-2 j+1} M_{j-\frac{1}{2}}^{(m)} G\left(j-\frac{1}{2}\right)\right)} \\
& =Y\left(\operatorname { e x p } \left(-\sum_{k=-1}^{\infty} \sum_{j \in \mathbb{Z}_{+}}\left(\begin{array}{l}
j+1 \\
k+1
\end{array}\right) t^{j}\left(a^{(m)}\right)^{-2 j} x^{j-k}\right.\right. \\
& \quad\left(\left(A_{j}^{(m)}+2\left(\frac{j-k}{j+1}\right) t^{-\frac{1}{2}} a^{(m)} x^{-1} \varphi M_{j-\frac{1}{2}}^{(m)}\right) L(k)\right. \\
& \left.\left.\left.+x^{-1}\left(\left(\frac{j-k}{j+1}\right) t^{-\frac{1}{2}} a^{(m)} M_{j-\frac{1}{2}}^{(m)}+\varphi \frac{(j-k)}{2} A_{j}^{(m)}\right) G\left(k+\frac{1}{2}\right)\right)\right) u,(x, \varphi)\right)
\end{aligned}
$$

By this equality, the proof of (7.11) is reduced to the proof of the following bracket formula

$$
\begin{aligned}
{\left[\sum_{j \in \mathbb{Z}_{+}}\left(t^{j}\left(a^{(m)}\right)^{-2 j} A_{j}^{(m)} L(j)+t^{j-\frac{1}{2}}\left(a^{(m)}\right)^{-2 j+1} M_{j-\frac{1}{2}}^{(m)} G\left(j-\frac{1}{2}\right)\right), Y(u,(x, \varphi))\right] } \\
(7.19)=Y\left(\sum_{k=-1}^{\infty} \sum_{j \in \mathbb{Z}_{+}}\left(\begin{array}{l}
j+1 \\
k+1
\end{array}\right) t^{j}\left(a^{(m)}\right)^{-2 j} x^{j-k}\right. \\
\quad\left(\left(A_{j}^{(m)}+2\left(\frac{j-k}{j+1}\right) t^{-\frac{1}{2}} a^{(m)} x^{-1} \varphi M_{j-\frac{1}{2}}^{(m)}\right) L(k)\right. \\
\left.\left.+x^{-1}\left(\left(\frac{j-k}{j+1}\right) t^{-\frac{1}{2}} a^{(m)} M_{j-\frac{1}{2}}^{(m)}+\varphi \frac{(j-k)}{2} A_{j}^{(m)}\right) G\left(k+\frac{1}{2}\right)\right) u,(x, \varphi)\right)
\end{aligned}
$$

From (5.14), we have

$$
\begin{aligned}
& {\left[Y\left(\tau,\left(x_{1}, \varphi_{1}\right)\right), Y(u,(x, \varphi))\right]} \\
& \quad=\operatorname{Res}_{x_{0}} x^{-1} \delta\left(\frac{x_{1}-x_{0}-\varphi_{1} \varphi}{x}\right) Y\left(Y\left(\tau,\left(x_{0}, \varphi_{1}-\varphi\right)\right) u,(x, \varphi)\right) .
\end{aligned}
$$

Let

$$
\begin{aligned}
& l_{t}\left(x_{1}\right)=\sum_{j \in \mathbb{Z}_{+}} t^{j}\left(a^{(m)}\right)^{-2 j} A_{j}^{(m)} x_{1}^{j+1} \\
& g_{t}\left(x_{1}\right)=\sum_{j \in \mathbb{Z}_{+}} t^{j-\frac{1}{2}}\left(a^{(m)}\right)^{-2 j+1} M_{j-\frac{1}{2}}^{(m)} x_{1}^{j} .
\end{aligned}
$$


Then

$$
\begin{aligned}
\varphi_{1} \operatorname{Res}_{x_{1}} l_{t}\left(x_{1}\right) Y\left(\omega,\left(x_{1}, \varphi_{1}\right)\right) & =\varphi_{1} \sum_{j \in \mathbb{Z}_{+}} t^{j}\left(a^{(m)}\right)^{-2 j} A_{j}^{(m)} L(j) \\
\varphi_{1} \operatorname{Res}_{x_{1}} g_{t}\left(x_{1}\right) Y\left(\tau,\left(x_{1}, \varphi_{1}\right)\right) & =\varphi_{1} \sum_{j \in \mathbb{Z}_{+}} t^{j-\frac{1}{2}}\left(a^{(m)}\right)^{-2 j+1} M_{j-\frac{1}{2}}^{(m)} G\left(j-\frac{1}{2}\right),
\end{aligned}
$$

and by the $G(-1 / 2)$-derivative property (5.12), we have

$$
\begin{aligned}
Y\left(\omega,\left(x_{1}, \varphi_{1}\right)\right) & =Y\left(\frac{1}{2} G\left(-\frac{1}{2}\right) \tau,\left(x_{1}, \varphi_{1}\right)\right) \\
& =\frac{1}{2}\left(\frac{\partial}{\partial \varphi_{1}}+\varphi_{1} \frac{\partial}{\partial x_{1}}\right) Y\left(\tau,\left(x_{1}, \varphi_{1}\right)\right) .
\end{aligned}
$$

Thus

$$
\begin{gathered}
\varphi_{1}\left[\sum_{j \in \mathbb{Z}_{+}}\left(t^{j}\left(a^{(m)}\right)^{-2 j} A_{j}^{(m)} L(j)+t^{j-\frac{1}{2}}\left(a^{(m)}\right)^{-2 j+1} M_{j-\frac{1}{2}}^{(m)} G\left(j-\frac{1}{2}\right)\right), Y(u,(x, \varphi))\right] \\
=\varphi_{1} \operatorname{Res}_{x_{1}}\left[\left(\frac{1}{2} l_{t}\left(x_{1}\right)\left(\frac{\partial}{\partial \varphi_{1}}+\varphi_{1} \frac{\partial}{\partial x_{1}}\right)+g_{t}\left(x_{1}\right)\right) Y\left(\tau,\left(x_{1}, \varphi_{1}\right)\right), Y(u,(x, \varphi))\right] \\
=\varphi_{1} \operatorname{Res}_{x_{1}}\left(\left(\frac{1}{2} l_{t}\left(x_{1}\right)\left(\frac{\partial}{\partial \varphi_{1}}+\varphi_{1} \frac{\partial}{\partial x_{1}}\right)+g_{t}\left(x_{1}\right)\right)\right. \\
\left.\operatorname{Res}_{x_{0}} x^{-1} \delta\left(\frac{x_{1}-x_{0}-\varphi_{1} \varphi}{x}\right) Y\left(Y\left(\tau,\left(x_{0}, \varphi_{1}-\varphi\right)\right) u,(x, \varphi)\right)\right) \\
=Y\left(\varphi_{1} \operatorname{Res}_{x_{1}} \operatorname{Res}_{x_{0}}\left(\frac{1}{2} l_{t}\left(x_{1}\right)\left(\frac{\partial}{\partial \varphi_{1}}+\varphi_{1} \frac{\partial}{\partial x_{1}}\right)+g_{t}\left(x_{1}\right)\right)\right. \\
\left.x^{-1} \delta\left(\frac{x_{1}-x_{0}-\varphi_{1} \varphi}{x}\right) Y\left(\tau,\left(x_{0}, \varphi_{1}-\varphi\right)\right) u,(x, \varphi)\right) .
\end{gathered}
$$

But

$$
\begin{gathered}
\varphi_{1} \operatorname{Res}_{x_{1}} \operatorname{Res}_{x_{0}}\left(\frac{1}{2} l_{t}\left(x_{1}\right)\left(\frac{\partial}{\partial \varphi_{1}}+\varphi_{1} \frac{\partial}{\partial x_{1}}\right)+g_{t}\left(x_{1}\right)\right) \\
x^{-1} \delta\left(\frac{x_{1}-x_{0}-\varphi_{1} \varphi}{x}\right) Y\left(\tau,\left(x_{0}, \varphi_{1}-\varphi\right)\right) \\
=\varphi_{1} \operatorname{Res}_{x_{1}} \operatorname{Res}_{x_{0}}\left(\frac{1}{2} l_{t}\left(x_{1}\right)\left(\frac{\partial}{\partial \varphi_{1}}+\varphi_{1} \frac{\partial}{\partial x_{1}}\right)+g_{t}\left(x_{1}\right)\right) \\
=\varphi_{1} \operatorname{Res}_{x_{1}} \operatorname{Res}_{x_{0}}\left(\frac { 1 } { 2 } l _ { t } ( x _ { 1 } ) \left(\frac{\partial}{\partial \varphi_{1}} x_{1}^{-1} \delta\left(\frac{x+x_{0}+\varphi_{1} \varphi}{x_{1}}\right) Y\left(\tau,\left(x_{0}, \varphi_{1}-\varphi\right)\right)\right.\right. \\
+\frac{1}{2} l_{t}\left(x_{1}\right) x_{1}^{-1} \delta\left(\frac{x+x_{1}}{x_{1}}\right) \frac{\partial}{\partial \varphi_{1}} Y\left(\tau,\left(x_{0}, \varphi_{1}\right)\right) \\
\left.+g_{t}\left(x_{1}\right) x_{1}^{-1} \delta\left(\frac{x+x_{0}}{x_{1}}\right) Y\left(\tau,\left(x_{0},-\varphi\right)\right)\right)
\end{gathered}
$$




$$
\begin{aligned}
& =\varphi_{1} \operatorname{Res}_{x_{1}} \operatorname{Res}_{x_{0}}\left(\left(\sum_{n \in \mathbb{Z}} n\left(x+x_{0}\right)^{n-1} \varphi x_{1}^{-n-1}\right)\left(\frac{1}{2} \sum_{j \in \mathbb{Z}_{+}} t^{j}\left(a^{(m)}\right)^{-2 j} A_{j}^{(m)} x_{1}^{j+1}\right)\right. \\
& \left(\sum_{k \in \mathbb{Z}} G\left(k+\frac{1}{2}\right) x_{0}^{-k-2}\right)+\left(\sum_{n \in \mathbb{Z}}\left(x+x_{0}\right)^{n} x_{1}^{-n-1}\right)\left(\frac{1}{2} \sum_{j \in \mathbb{Z}_{+}} t^{j}\left(a^{(m)}\right)^{-2 j} A_{j}^{(m)} x_{1}^{j+1}\right) \\
& \left(2 \sum_{k \in \mathbb{Z}} L(k) x_{0}^{-k-2}\right)+\left(\sum_{n \in \mathbb{Z}}\left(x+x_{0}\right)^{n} x_{1}^{-n-1}\right)\left(\sum_{j \in \mathbb{Z}_{+}} t^{j-\frac{1}{2}}\left(a^{(m)}\right)^{-2 j+1} M_{j-\frac{1}{2}}^{(m)} x_{1}^{j}\right) \\
& \left.\left(\sum_{k \in \mathbb{Z}} G\left(k+\frac{1}{2}\right) x_{0}^{-k-2}-2 \varphi L(k) x_{0}^{-k-2}\right)\right) \\
& =\varphi_{1} \operatorname{Res}_{x_{0}}\left(\sum _ { l \in \mathbb { N } } \sum _ { k \in \mathbb { Z } } \sum _ { j \in \mathbb { Z } _ { + } } \left((j+1)\left(\begin{array}{l}
j \\
l
\end{array}\right) x^{j-l} x_{0}^{l} \frac{\varphi}{2} t^{j}\left(a^{(m)}\right)^{-2 j} A_{j}^{(m)} G\left(k+\frac{1}{2}\right) x_{0}^{-k-2}\right.\right. \\
& +\left(\begin{array}{c}
j+1 \\
l
\end{array}\right) x^{j-l+1} x_{0}^{l} t^{j}\left(a^{(m)}\right)^{-2 j} A_{j}^{(m)} L(k) x_{0}^{-k-2} \\
& \left.\left.+\left(\begin{array}{l}
j \\
l
\end{array}\right) x^{j-l} x_{0}^{l} t^{j-\frac{1}{2}}\left(a^{(m)}\right)^{-2 j+1} M_{j-\frac{1}{2}}^{(m)}\left(G\left(k+\frac{1}{2}\right)-2 \varphi L(k)\right) x_{0}^{-k-2}\right)\right) \\
& =\varphi_{1} \sum_{l \in \mathbb{N}} \sum_{j \in \mathbb{Z}_{+}}\left(\frac{(j+1)}{2}\left(\begin{array}{l}
j \\
l
\end{array}\right) x^{j-l} \varphi t^{j}\left(a^{(m)}\right)^{-2 j} A_{j}^{(m)} G\left(l-\frac{1}{2}\right)\right. \\
& +\left(\begin{array}{c}
j+1 \\
l
\end{array}\right) x^{j-l+1} t^{j}\left(a^{(m)}\right)^{-2 j} A_{j}^{(m)} L(l-1) \\
& \left.+\left(\begin{array}{l}
j \\
l
\end{array}\right) x^{j-l} t^{j-\frac{1}{2}}\left(a^{(m)}\right)^{-2 j+1} M_{j-\frac{1}{2}}^{(m)}\left(G\left(l-\frac{1}{2}\right)-2 \varphi L(l-1)\right)\right) \\
& =\varphi_{1} \sum_{k=-1}^{\infty} \sum_{j \in \mathbb{Z}_{+}}\left(\left(\begin{array}{l}
j+1 \\
k+1
\end{array}\right) t^{j}\left(a^{(m)}\right)^{-2 j} x^{j-k}\right. \\
& \left(A_{j}^{(m)}+2\left(\frac{j-k}{j+1}\right) x^{-1} t^{-\frac{1}{2}} a^{(m)} \varphi M_{j-\frac{1}{2}}^{(m)}\right) L(k) \\
& \left.+\left(\begin{array}{c}
j \\
k+1
\end{array}\right) t^{j}\left(a^{(m)}\right)^{-2 j} x^{j-k-1}\left(\frac{(j+1)}{2} \varphi A_{j}^{(m)}+t^{-\frac{1}{2}} a^{(m)} M_{j-\frac{1}{2}}^{(m)}\right) G\left(k+\frac{1}{2}\right)\right) .
\end{aligned}
$$

This finishes the proof of (7.14) thus concluding the proof of the sewing axiom for case (b).

(g) Let $n \geq 2$ and fix $m \in \mathbb{Z}_{+}$. Assume the sewing axiom holds for all $Q_{1} \in$ $S K(m), Q_{2} \in S K(n-1)$. We show the sewing axiom holds for all $Q_{1} \in S K(m)$, $Q_{2} \in S K(n)$. Let

$$
\begin{gathered}
Q_{1}=\left(\left(z_{1}, \theta_{1}\right), \ldots,\left(z_{m-1}, \theta_{m-1}\right) ;\left(A^{(0)}, M^{(0)}\right),\left(a^{(1)}, A^{(1)}, M^{(1)}\right), \ldots,\right. \\
\left.\left(a^{(m)}, A^{(m)}, M^{(m)}\right)\right), \\
Q_{2}=\left(\left(z_{1}^{\prime}, \theta_{1}^{\prime}\right), \ldots,\left(z_{n-1}^{\prime}, \theta_{n-1}^{\prime}\right) ;\left(B^{(0)}, N^{(0)}\right),\left(b^{(1)}, B^{(1)}, N^{(1)}\right), \ldots,\right. \\
\left.\left(b^{(n)}, B^{(n)}, N^{(n)}\right)\right) .
\end{gathered}
$$


Then since $\nu_{m+n-1}^{Y}\left(Q_{1} i_{i} \infty_{0} Q_{2}\right)$ and each term in the series $\left(\nu_{m}^{Y}\left(Q_{1}\right)_{i} *_{0} \nu_{n}^{Y}\left(Q_{2}\right)\right)_{t^{1 / 2}}$ are analytic in $\left(z_{1}^{\prime}\right)_{B}, \ldots,\left(z_{n-1}^{\prime}\right)_{B}$, we need only prove the sewing axiom when

$$
\left|\left(z_{1}^{\prime}\right)_{B}\right|, \ldots,\left|\left(z_{n-2}^{\prime}\right)_{B}\right|<\left|\left(z_{n-1}^{\prime}\right)_{B}\right| \text {. }
$$

In this case $Q_{2}=Q_{2}^{\prime}{ }_{n-1} \infty_{0} Q_{2}^{\prime \prime}$ where

$$
\begin{aligned}
Q_{2}^{\prime}=\left(\left(z_{1}^{\prime}, \theta_{1}^{\prime}\right), \ldots,\left(z_{n-2}^{\prime}, \theta_{n-2}^{\prime}\right) ;\left(B^{(0)}, N^{(0)}\right),\left(b^{(1)}, B^{(1)}, N^{(1)}\right), \ldots,\right. \\
\left.\left(b^{(n-2)}, B^{(n-2)}, N^{(n-2)}\right),(1, \mathbf{0})\right) \in S K(n-1)
\end{aligned}
$$

and

$$
Q_{2}^{\prime \prime}=\left(\left(z_{n-1}^{\prime}, \theta_{n-1}^{\prime}\right) ; \mathbf{0},\left(b^{(n-1)}, B^{(n-1)}, N^{(n-1)}\right),\left(b^{(n)}, B^{(n)}, N^{(n)}\right)\right) \in S K(2) .
$$

By our inductive assumption

$$
\left(\nu_{m}^{Y}\left(Q_{1}\right)_{i} *_{0} \quad \nu_{n-1}^{Y}\left(Q_{2}^{\prime}\right)\right) e^{-\Gamma\left(a^{(i)}, A^{(i)}, M^{(i)}, B^{(0)}, N^{(0)}\right) c}=\nu_{m+n-2}^{Y}\left(Q_{1}{ }_{i} \infty_{0} Q_{2}^{\prime}\right),
$$

and by step (f)

$$
\nu_{m+n-2}^{Y}\left(Q_{1 i} \infty_{0} Q_{2}^{\prime}\right)_{m+n-2} *_{0} \nu_{2}^{Y}\left(Q_{2}^{\prime \prime}\right)=\nu_{m+n-1}^{Y}\left(\left(Q_{1}{ }_{i} \infty_{0} Q_{2}^{\prime}\right)_{m+n-2} \infty_{0} Q_{2}^{\prime \prime}\right) .
$$

Thus using the associativity of sewing and the $t^{1 / 2}$-contraction, Propositions 2.5 and 4.1 and Proposition 7.1 we have

$$
\begin{aligned}
& \left(\nu_{m}^{Y}\left(Q_{1}\right)_{i} *_{0} \nu_{n}^{Y}\left(Q_{2}\right)\right) e^{-\Gamma\left(a^{(i)}, A^{(i)}, M^{(i)}, B^{(0)}, N^{(0)}\right) c} \\
& \quad=\nu_{m}^{Y}\left(Q_{1}\right)_{i} *_{0}\left(\nu_{n-1}^{Y}\left(Q_{2}^{\prime}\right)_{n-1} *_{0} \nu_{2}^{Y}\left(Q_{2}^{\prime \prime}\right)\right) e^{-\Gamma\left(a^{(i)}, A^{(i)}, M^{(i)}, B^{(0)}, N^{(0)}\right) c} \\
& \quad=\left(\left(\nu_{m}^{Y}\left(Q_{1}\right)_{i} *_{0} \nu_{n-1}^{Y}\left(Q_{2}^{\prime}\right)\right)_{i+n-2} *_{0} \nu_{2}^{Y}\left(Q_{2}^{\prime \prime}\right)\right) e^{-\Gamma\left(a^{(i)}, A^{(i)}, M^{(i)}, B^{(0)}, N^{(0)}\right) c} \\
& \quad=\nu_{m+n-1}^{Y}\left(\left(Q_{1 i} \infty_{0} Q_{2}^{\prime}\right)_{i+n-2} \infty_{0} Q_{2}^{\prime \prime}\right) \\
& =\nu_{m+n-1}^{Y}\left(Q_{1 i} \infty_{0}\left(Q_{2}^{\prime}{ }_{n-1} \infty_{0} Q_{2}^{\prime \prime}\right)\right) \\
& =\nu_{m+n-1}^{Y}\left(Q_{1 i} \infty_{0} Q_{2}\right) .
\end{aligned}
$$

(h) Finally, assuming the sewing axiom holds for $Q_{1} \in S K(m-1)$ with $m \geq 2$ and $Q_{2} \in S K(n)$ for all $n$, we show that it holds for $Q_{1} \in S K(m)$ and $Q_{2} \in S K(n)$. Let

$$
\begin{gathered}
Q_{1}=\left(\left(z_{1}, \theta_{1}\right), \ldots,\left(z_{m-1}, \theta_{m-1}\right) ;\left(A^{(0)}, M^{(0)}\right),\left(a^{(1)}, A^{(1)}, M^{(1)}\right), \ldots,\right. \\
\left.\left(a^{(m)}, A^{(m)}, M^{(m)}\right)\right), \\
Q_{2}=\left(\left(z_{1}^{\prime}, \theta_{1}^{\prime}\right), \ldots,\left(z_{n-1}^{\prime}, \theta_{n-1}^{\prime}\right) ;\left(B^{(0)}, N^{(0)}\right),\left(b^{(1)}, B^{(1)}, N^{(1)}\right), \ldots,\right. \\
\left.\left(b^{(n)}, B^{(n)}, N^{(n)}\right)\right) .
\end{gathered}
$$

Then since $\nu_{m+n-1}^{Y}\left(Q_{1 i} \infty_{0} Q_{2}\right)$ and every term in the series $\left(\nu_{m}^{Y}\left(Q_{1}\right)_{i} *_{0} \nu_{n}^{Y}\left(Q_{2}\right)\right)_{t^{1 / 2}}$ is analytic in $\left(z_{1}\right)_{B}, \ldots,\left(z_{m-1}\right)_{B}$, we need only prove the sewing axiom when

$$
\left|\left(z_{1}\right)_{B}\right|, \ldots,\left|\left(z_{m-2}\right)_{B}\right|<\left|\left(z_{m-1}\right)_{B}\right| \text {. }
$$

In this case $Q_{1}=Q_{1}^{\prime}{ }_{m-1} \infty_{0} Q_{1}^{\prime \prime}$ where

$$
\begin{aligned}
Q_{1}^{\prime}=\left(\left(z_{1}, \theta_{1}\right), \ldots,\left(z_{m-2}, \theta_{m-2}\right)\right. & ;\left(A^{(0)}, M^{(0)}\right),\left(a^{(1)}, A^{(1)}, M^{(1)}\right), \ldots, \\
& \left.\left(a^{(m-2)}, A^{(m-2)}, M^{(m-2)}\right),(1, \mathbf{0})\right) \in S K(m-1)
\end{aligned}
$$

and

$$
Q_{1}^{\prime \prime}=\left(\left(z_{m-1}, \theta_{m-1}\right) ; \mathbf{0},\left(a^{(m-1)}, A^{(m-1)}, M^{(m-1)}\right),\left(a^{(m)}, A^{(m)}, M^{(m)}\right)\right) \in S K(2) .
$$


We have three cases:

(i) If $i=m$, then

$$
Q_{1 i} \infty_{0} Q_{2}=\left(Q_{1 m-1}^{\prime} \infty_{0} Q_{1}^{\prime \prime}\right)_{m} \infty_{0} Q_{2}=Q_{1 m-1}^{\prime} \infty_{0}\left(Q_{12}^{\prime \prime} \infty_{0} Q_{2}\right) .
$$

For $m>2$, the sewing axiom follows by the inductive assumption, associativity of sewing and the $t^{1 / 2}$-contraction and Proposition 7.1 For $m=2$ the sewing axiom follows by special case (c), by (g) (the induction on $n$ ), the associativity of sewing and the $t^{1 / 2}$-contraction and Proposition 7.1

(ii) If $i=m-1$, then

$$
Q_{1 i} \infty_{0} Q_{2}=\left(Q_{1 m-1}^{\prime} \infty_{0} Q_{1}^{\prime \prime}\right)_{m-1} \infty_{0} Q_{2}=Q_{1 m-1}^{\prime} \infty_{0}\left(Q_{1}^{\prime \prime}{ }_{1} \infty_{0} Q_{2}\right) .
$$

For $m>2$, the sewing axiom follows by the inductive assumption, associativity of sewing and the $t^{1 / 2}$-contraction and Proposition [7.1 For $m=2$ the sewing axiom follows by special case (e), by (g) (the induction on $n$ ), the associativity of sewing and the $t^{1 / 2}$-contraction and Proposition 7.1

(iii) If $i \neq m, m-1$, then

$$
Q_{1 i} \infty_{0} Q_{2}=\left(Q_{1 m-1}^{\prime} \infty_{0} Q_{1}^{\prime \prime}\right)_{i} \infty_{0} Q_{2}=\left(Q_{1}^{\prime}{ }_{i} \infty_{0} Q_{2}\right)_{m+n-2} \infty_{0} Q_{1}^{\prime \prime},
$$

and the sewing axiom follows by the inductive assumption, special case (f), the associativity of sewing and the $t^{1 / 2}$-contraction and Proposition 7.1

\section{The isomorphism BetWeEn the CATEgory of $N=1$ SG-VOSAs AND THE CATEGORY OF $N=1$ NS-VOSAS}

In this section, we prove the equivalence of the notions of $N=1$ NS-VOSA with odd formal variables and $N=1$ SG-VOSA. By Theorem 5.3 in B3, this then implies the equivalence of $N=1$ NS-VOSA without odd formal variables and $N=1$ SG-VOSAs. Again, since $N=1$ NS- (resp., SG-) VOSAs are isomorphic if taken over different subalgebras of the underlying Grassmann algebra $\Lambda_{\infty}$, we suppress the details of which subalgebras are actually acting non-trivially.

Let $\mathbf{S G}(c)$ be the category of $N=1$ SG-VOSAs with central charge $c$. Let $\mathbf{S V}(\varphi, c)$ be the category of $N=1$ NS-VOSAs with odd formal variables and central charge $c$, and let $\mathbf{S V}(c)$ be the category of $N=1$ NS-VOSAs without odd formal variables and with central charge $c$. Let $1_{S V_{\varphi}}$ and $1_{S G}$ be the identity functors on $\mathbf{S V}(\varphi, c)$ and $\mathbf{S G}(c)$, respectively. The following theorem is the main result of this paper.

Theorem 8.1. Assume that Conjecture 3.5 is true. For any $c \in \mathbb{C}$, the two categories $\mathbf{S V}(\varphi, c)$ and $\mathbf{S G}(c)$ are isomorphic (and hence $\mathbf{S V}(c)$ and $\mathbf{S G}(c)$ are isomorphic). That is there exist two functors $F_{S V}: \mathbf{S V}(\varphi, c) \rightarrow \mathbf{S G}(c)$ and $F_{S G}: \mathbf{S G}(c) \rightarrow \mathbf{S V}(\varphi, c)$ such that $F_{S V} \circ F_{S G}=1_{S G}$ and $F_{S G} \circ F_{S V}=1_{S V_{\varphi}}$.

Proof. We begin by defining $F_{S V}$ on objects by $F_{S V}(V, Y(\cdot,(x, \varphi)), \mathbf{1}, \tau)=\left(V, \nu^{Y}\right)$. Proposition 7.2 shows that $F_{S V}$ takes objects in $\mathbf{S V}(\varphi, c)$ to objects in $\mathbf{S G}(c)$.

Let $\gamma:\left(V_{1}, Y_{1}(\cdot,(x, \varphi)), \mathbf{1}_{1}, \tau_{1}\right) \rightarrow\left(V_{2}, Y_{2}(\cdot,(x, \varphi)), \mathbf{1}_{2}, \tau_{2}\right)$ be an $N=1$ NSVOSA homomorphism. Then for $Q \in S K(n)$ given by

$Q=\left(\left(z_{1}, \theta_{1}\right), \ldots,\left(z_{n-1}, \theta_{n-1}\right) ;\left(A^{(0)}, M^{(0)}\right),\left(a^{(1)}, A^{(1)}, M^{(1)}\right), \ldots,\left(a^{(n)}, A^{(n)}, M^{(n)}\right)\right)$, and for $\bar{\gamma}: \bar{V}_{1} \longrightarrow \bar{V}_{2}$ the unique extension of $\gamma$, we have $\left\langle v^{\prime}, \bar{\gamma} \circ \nu_{n}^{Y_{1}}(Q)\left(v_{1} \otimes \cdots \otimes v_{n}\right)\right\rangle=$ 


$$
\begin{gathered}
=\iota_{1 \cdots n-1}^{-1}\left\langle e^{-\sum_{j \in \mathbb{Z}_{+}}\left(A_{j}^{(0)} L^{\prime}(j)+M_{j-\frac{1}{2}}^{(0)} G^{\prime}\left(j-\frac{1}{2}\right)\right)} v^{\prime},\right. \\
\bar{\gamma}\left(Y_{1}\left(e^{-\sum_{j \in \mathbb{Z}_{+}}\left(A_{j}^{(1)} L(j)+M_{j-\frac{1}{2}}^{(1)} G\left(j-\frac{1}{2}\right)\right)} \cdot\left(a^{(1)}\right)^{-2 L(0)} \cdot v_{1},\left(x_{1}, \varphi_{1}\right)\right) \cdots\right. \\
Y_{1}\left(e^{-\sum_{j \in \mathbb{Z}_{+}}\left(A_{j}^{(n-1)} L(j)+M_{j-\frac{1}{2}}^{(n-1)} G\left(j-\frac{1}{2}\right)\right)} \cdot\left(a^{(n-1)}\right)^{-2 L(0)} \cdot v_{n-1},\right. \\
\left.\left.\left.\left(x_{n-1}, \varphi_{n-1}\right)\right) \cdot e^{-\sum_{j \in \mathbb{Z}_{+}}\left(A_{j}^{(n)} L(j)+M_{j-\frac{1}{2}}^{(n)} G\left(j-\frac{1}{2}\right)\right)} \cdot\left(a^{(n)}\right)^{-2 L(0)} \cdot v_{n}\right)\right\rangle\left.\right|_{\left(x_{i}, \varphi_{i}\right)=\left(z_{i}, \theta_{i}\right)} \\
=\iota_{1 \cdots n-1}^{-1}\left\langle e^{-\sum_{j \in \mathbb{Z}_{+}}\left(A_{j}^{(0)} L^{\prime}(j)+M_{j-\frac{1}{2}}^{(0)} G^{\prime}\left(j-\frac{1}{2}\right)\right)} v^{\prime},\right. \\
Y_{2}\left(e^{-\sum_{j \in \mathbb{Z}_{+}}\left(A_{j}^{(1)} L(j)+M_{j-\frac{1}{2}}^{(1)} G\left(j-\frac{1}{2}\right)\right)} \cdot\left(a^{(1)}\right)^{-2 L(0)} \cdot \gamma\left(v_{1}\right),\left(x_{1}, \varphi_{1}\right)\right) \cdots \\
Y_{2}\left(e^{-\sum_{j \in \mathbb{Z}_{+}}\left(A_{j}^{(n-1)} L(j)+M_{j-\frac{1}{2}}^{(n-1)} G\left(j-\frac{1}{2}\right)\right)} \cdot\left(a^{(n-1)}\right)^{-2 L(0)} \cdot \gamma\left(v_{n-1}\right),\right. \\
\left.\left(x_{n-1}, \varphi_{n-1}\right)\right) \cdot e^{-\sum_{j \in \mathbb{Z}_{+}}\left(A_{j}^{(n)} L(j)+M_{j-\frac{1}{2}}^{(n)} G\left(j-\frac{1}{2}\right)\right)} . \\
=\left\langle a^{\prime}, \nu_{n}^{Y_{2}}(Q) \circ \gamma^{\otimes n}\left(v_{1} \otimes \cdots \otimes v_{n}\right)\right\rangle .
\end{gathered}
$$

Thus defining $F_{S V}(\gamma)=\gamma$, we have that $F_{S V}(\gamma)$ is a homomorphism of $N=1$ SG-VOSAs, i.e., $F_{S V}$ takes morphisms in $\mathbf{S V}(\varphi, c)$ to morphisms in $\mathbf{S G}(c)$. By definition, $F_{S V}\left(\gamma_{1} \gamma_{2}\right)=\gamma_{1} \gamma_{2}=F_{S V}\left(\gamma_{1}\right) F_{S V}\left(\gamma_{2}\right)$, and $F_{S V}\left(i d_{(V, Y(\cdot,(x, \varphi)), \mathbf{1}, \tau)}\right)=$ $i d_{\left(V, \nu^{Y}\right)}$. Thus $F_{S V}$ is a functor.

We next define $F_{S G}(V, \nu)=\left(V, Y_{\nu}(\cdot,(x, \varphi)), \mathbf{1}_{\nu}, \tau_{\nu}\right)$. Proposition 6.1 shows that $F_{S G}$ takes objects in $\mathbf{S G}(c)$ to objects in $\mathbf{S V}(\varphi, c)$. Let $\gamma:\left(V_{1}, \nu\right) \rightarrow\left(V_{2}, \mu\right)$ be an $N=1$ SG-VOSA homomorphism. Then

$$
\begin{aligned}
\left.\left\langle v^{\prime}, \gamma\left(Y_{\nu}(u,(x, \varphi)) v\right)\right\rangle\right|_{(x, \varphi)=(z, \theta)} & \left\langle v^{\prime}, \bar{\gamma} \circ \nu_{2}((z, \theta) ; \mathbf{0},(1, \mathbf{0}),(1, \mathbf{0}))(u \otimes v)\right\rangle \\
& =\left\langle v^{\prime}, \mu_{2}((z, \theta) ; \mathbf{0},(1, \mathbf{0}),(1, \mathbf{0})) \gamma^{\otimes 2}(u \otimes v)\right\rangle \\
& =\left.\left\langle v^{\prime}, Y_{\mu}(\gamma(u),(x, \varphi)) \gamma(v)\right\rangle\right|_{(x, \varphi)=(z, \theta)}, \\
\left\langle v^{\prime}, \gamma\left(\mathbf{1}_{\nu}\right)\right\rangle & =\left\langle v^{\prime}, \bar{\gamma} \circ \nu_{0}(\mathbf{0})\right\rangle \\
& =\left\langle v^{\prime}, \mu_{0}(\mathbf{0})\right\rangle \\
& =\left\langle v^{\prime}, \gamma\left(\mathbf{1}_{\mu}\right)\right\rangle,
\end{aligned}
$$

and

$$
\begin{aligned}
\left\langle v^{\prime}, \gamma\left(\tau_{\nu}\right)\right\rangle & =\left\langle v^{\prime}, \bar{\gamma}\left(-\frac{\partial}{\partial \epsilon} \nu_{0}\left(\mathbf{0}, M\left(\epsilon, \frac{3}{2}\right)\right)\right)\right\rangle \\
& =-\left\langle v^{\prime}, \frac{\partial}{\partial \epsilon} \bar{\gamma} \circ \nu_{0}\left(\mathbf{0}, M\left(\epsilon, \frac{3}{2}\right)\right)\right\rangle \\
& =-\left\langle v^{\prime}, \frac{\partial}{\partial \epsilon} \mu_{0}\left(\mathbf{0}, M\left(\epsilon, \frac{3}{2}\right)\right)\right\rangle \\
& =\left\langle v^{\prime}, \gamma\left(\tau_{\mu}\right)\right\rangle
\end{aligned}
$$


for all $v^{\prime} \in V^{\prime}$. Thus defining $F_{S G}(\gamma)=\gamma$, we have that $F_{S G}(\gamma)$ is indeed an $N=1$ NS-VOSA homomorphism, i.e., $F_{S G}$ takes morphisms in SG $(c)$ to morphisms in $\mathbf{S V}(\varphi, c)$. By definition, $F_{S G}\left(\gamma_{1} \gamma_{2}\right)=\gamma_{1} \gamma_{2}=F_{S G}\left(\gamma_{1}\right) F_{S G}\left(\gamma_{2}\right)$, and $F_{S G}\left(i d_{(V, \nu)}\right)=$ $\left.i d_{\left(V, Y_{\nu}(\cdot,(x, \varphi)), \mathbf{1}_{\nu}, \tau_{\nu}\right)}\right)$. Thus $F_{S G}$ is a functor.

The fact that $F_{S V} \circ F_{S G}=1_{S G}$ and $F_{S G} \circ F_{S V}=1_{S V_{\varphi}}$ on morphisms is trivial. To show $F_{S V} \circ F_{S G}=1_{S G}$ on objects, we need to show that given an $N=1$ SG-VOSA, $(V, \nu)$, then $F_{S V} \circ F_{S G}(V, \nu)=\left(V, \nu^{Y_{\nu}}\right)=(V, \nu)$, i.e.,

$$
\begin{aligned}
& \left\langle v^{\prime}, \nu_{n}\left(\left(z_{1}, \theta_{1}\right), \ldots,\left(z_{n-1}, \theta_{n-1}\right) ;\left(A^{(0)}, M^{(0)}\right),\left(a^{(1)}, A^{(1)}, M^{(1)}\right), \ldots,\right.\right. \\
& \left.\left.\left(a^{(n)}, A^{(n)}, M^{(n)}\right)\right)\left(v_{1} \otimes \cdots \otimes v_{n}\right)\right\rangle \\
& =\iota_{1 \cdots n-1}^{-1}\left\langle e^{-\sum_{j \in \mathbb{Z}_{+}}\left(A_{j}^{(0)} L^{\prime}(j)+M_{j-\frac{1}{2}}^{(0)} G^{\prime}\left(j-\frac{1}{2}\right)\right)} v^{\prime}\right. \\
& Y_{\nu}\left(e^{-\sum_{j \in \mathbb{Z}_{+}}\left(A_{j}^{(1)} L(j)+M_{j-\frac{1}{2}}^{(1)} G\left(j-\frac{1}{2}\right)\right)} \cdot\left(a^{(1)}\right)^{-2 L(0)} \cdot v_{1},\left(x_{1}, \varphi_{1}\right)\right) \cdots \\
& Y_{\nu}\left(e^{-\sum_{j \in \mathbb{Z}_{+}}\left(A_{j}^{(n-1)} L(j)+M_{j-\frac{1}{2}}^{(n-1)} G\left(j-\frac{1}{2}\right)\right)} \cdot\left(a^{(n-1)}\right)^{-2 L(0)} \cdot v_{n-1},\left(x_{n-1}, \varphi_{n-1}\right)\right) . \\
& \left.\left.e^{-\sum_{j \in \mathbb{Z}_{+}}\left(A_{j}^{(n)} L(j)+M_{j-\frac{1}{2}}^{(n)} G\left(j-\frac{1}{2}\right)\right)} \cdot\left(a^{(n)}\right)^{-2 L(0)} \cdot v_{n}\right)\right\rangle\left.\right|_{\left(x_{i}, \varphi_{i}\right)=\left(z_{i}, \theta_{i}\right)} .
\end{aligned}
$$

Since both $(V, \nu)$ and $F_{S V} \circ F_{S G}(V, \nu)=\left(V, \nu^{Y_{\nu}}\right)$ are $N=1$ SG-VOSAs, they both satisfy the sewing axiom. Thus by Proposition [2.6] we need only prove (8.1) for $Q=(\mathbf{0}),\left(\left(A^{(0)}, M^{(0)}\right),\left(a^{(1)}, A^{(1)}, M^{(1)}\right)\right)$, and $((z, \theta) ; \mathbf{0},(1, \mathbf{0}),(1, \mathbf{0}))$. For $Q=(\mathbf{0})$ and $((z, \theta) ; \mathbf{0},(1, \mathbf{0}),(1, \mathbf{0}))$, equation (8.1) follows immediately from the definitions of $\mathbf{1}_{\nu}$ and $Y_{\nu}$. The equality

$$
\begin{aligned}
&\left\langle v^{\prime},\right.\left.\nu_{1}\left(\left(A^{(0)}, M^{(0)}\right),\left(a^{(1)}, A^{(1)}, M^{(1)}\right)\right)(v)\right\rangle \\
&=\left\langle e^{-\sum_{j \in \mathbb{Z}_{+}}\left(A_{j}^{(0)} L^{\prime}(j)+M_{j-\frac{1}{2}}^{(0)} G^{\prime}\left(j-\frac{1}{2}\right)\right)} v^{\prime}, e^{-\sum_{j \in \mathbb{Z}_{+}}\left(A_{j}^{(1)} L(j)+M_{j-\frac{1}{2}}^{(1)} G\left(j-\frac{1}{2}\right)\right)} .\right. \\
&\left.\left.\left(a^{(1)}\right)^{-2 L(0)} \cdot v\right)\right\rangle
\end{aligned}
$$

is equivalent to the following three equations

$$
\begin{aligned}
\left\langle v^{\prime}, \nu_{1}\left(\left(A^{(0)}, M^{(0)}\right),(1, \mathbf{0})\right)(v)\right\rangle & \left.=\left\langle e^{-\sum_{j \in \mathbb{Z}_{+}}\left(A_{j}^{(0)} L^{\prime}(j)+M_{j-\frac{1}{2}}^{(0)} G^{\prime}\left(j-\frac{1}{2}\right)\right)} v^{\prime}, v\right)\right\rangle \\
\left\langle v^{\prime}, \nu_{1}\left(\mathbf{0},\left(1, A^{(1)}, M^{(1)}\right)\right)(v)\right\rangle & \left.=\left\langle v^{\prime}, e^{-\sum_{j \in \mathbb{Z}_{+}}\left(A_{j}^{(1)} L(j)+M_{j-\frac{1}{2}}^{(1)} G\left(j-\frac{1}{2}\right)\right)} \cdot v\right)\right\rangle \\
\left\langle v^{\prime}, \nu_{1}\left(\mathbf{0},\left(a^{(1)}, \mathbf{0}\right)\right)(v)\right\rangle & \left.=\left\langle v^{\prime},\left(a^{(1)}\right)^{-2 L(0)} \cdot v\right)\right\rangle .
\end{aligned}
$$

The equality (8.4) follows immediately from the grading axiom. By the supermeromorphicity axiom, $\left\langle v^{\prime}, \nu_{1}\left(\mathbf{0},\left(1, A^{(1)}, M^{(1)}\right)\right)(v)\right\rangle$ is a polynomial in the $A_{j}^{(1)}$ 's and $M_{j-1 / 2}^{(1)}$ 's for $j \in \mathbb{Z}_{+}$. Thus $\left\langle v^{\prime}, \nu_{1}\left(\mathbf{0},\left(1, A^{(1)}, M^{(1)}\right)\right)(v)\right\rangle$ is well defined for any $\left(A^{(1)}, M^{(1)}\right) \in \bigwedge_{\infty}^{\infty}$. But since the series $\left(A^{(1)}, M^{(1)}\right)$ is not necessarily in $\mathcal{H}$, the formal series $\tilde{E}\left(A^{(1)}, M^{(1)}\right)(x, \varphi)$ is not necessarily convergent. In B4], such superspheres as $\left(\mathbf{0},\left(1, A^{(1)}, M^{(1)}\right)\right)$ were called generalized superspheres with tubes, and the space of such formal superspheres was denoted $\tilde{S K}(1)$. However, since $\left\langle v^{\prime}, \nu_{1}\left(\mathbf{0},\left(1, t\left(A^{(1)}, M^{(1)}\right)\right)\right)(v)\right\rangle$ is linear in $v$ and $v^{\prime}$, there exists a linear operator 
$R(t)$ from $V$ to $\bar{V}$ such that

$$
\left\langle v^{\prime}, \nu_{1}\left(\mathbf{0},\left(1, t\left(A^{(1)}, M^{(1)}\right)\right)(v)\right\rangle=\left\langle v^{\prime}, R(t) v\right\rangle .\right.
$$

We first show that, in fact, $R(t)$ is an operator from $V$ to itself. Using the sewing axiom and (8.4), we have

$$
\begin{aligned}
\left\langle v^{\prime}\right. & , \nu_{1}\left(\left(\mathbf{0},\left(t_{1}^{\frac{1}{2}}, \mathbf{0}\right)\right){ }_{1} \infty_{0}\left(\mathbf{0},\left(1, t\left(A^{(1)}, M^{(1)}\right)\right)\right)(v)\right\rangle \\
& =\left\langle v^{\prime},\left(\nu_{1}\left(\mathbf{0},\left(t_{1}^{\frac{1}{2}}, \mathbf{0}\right)\right)_{1} *_{0} \nu_{1}\left(\mathbf{0},\left(1, t\left(A^{(1)}, M^{(1)}\right)\right)\right)(v)\right\rangle\right. \\
& =\sum_{k \in \frac{1}{2} \mathbb{Z}} \sum_{i^{(k)}=1}^{\operatorname{dim} V_{(k)}}\left\langle v^{\prime}, \nu_{1}\left(\mathbf{0},\left(t_{1}^{\frac{1}{2}}, \mathbf{0}\right)\right) e_{i^{(k)}}^{(k)}\right\rangle\left\langle\left(e_{i^{(k)}}^{(k)}\right)^{*}, \nu_{1}\left(\mathbf{0},\left(1, t\left(A^{(1)}, M^{(1)}\right)\right)(v)\right\rangle\right. \\
& =\sum_{k \in \frac{1}{2} \mathbb{Z}} \sum_{i^{(k)}=1}^{\operatorname{dim} V_{(k)}}\left\langle v^{\prime}, t_{1}^{-L(0)} e_{i(k)}^{(k)}\right\rangle\left\langle\left(e_{i(k)}^{(k)}\right)^{*}, R(t) v\right\rangle \\
& =\left\langle v^{\prime}, t_{1}^{-L(0)} R(t) v\right\rangle \\
& =\left\langle t_{1}^{-L^{\prime}(0)} v^{\prime}, R(t) v\right\rangle .
\end{aligned}
$$

On the other hand, from

$$
\begin{aligned}
\left(\mathbf{0},\left(t_{1}^{\frac{1}{2}}, \mathbf{0}\right)\right){ }_{1} \infty_{0}(\mathbf{0},(1, t & \left.\left(A^{(1)}, M^{(1)}\right)\right) \\
& =\left(\mathbf{0},\left(1,\left\{t t_{1}^{j} A_{j}^{(1)}, t t_{1}^{j-\frac{1}{2}} M_{j-\frac{1}{2}}^{(1)}\right\}_{j \in \mathbb{Z}_{+}}\right)\right)_{1} \infty_{0}\left(\mathbf{0},\left(t_{1}^{\frac{1}{2}}, \mathbf{0}\right)\right),
\end{aligned}
$$

we have

$$
\begin{aligned}
& \left\langle v^{\prime}, \nu_{1}\left(\left(\mathbf{0},\left(t_{1}^{\frac{1}{2}}, \mathbf{0}\right)\right)_{1} \infty_{0}\left(\mathbf{0},\left(1, t\left(A^{(1)}, M^{(1)}\right)\right)\right)(v)\right\rangle\right. \\
& =\left\langle v^{\prime}, \nu_{1}\left(\left(\mathbf{0},\left(1,\left\{t t_{1}^{j} A_{j}^{(1)}, t t_{1}^{j-\frac{1}{2}} M_{j-\frac{1}{2}}^{(1)}\right\}_{j \in \mathbb{Z}_{+}}\right)\right)_{1} \infty_{0}\left(\mathbf{0},\left(t_{1}^{\frac{1}{2}}, \mathbf{0}\right)\right)\right)(v)\right\rangle \\
& =\left\langle v^{\prime},\left(\nu_{1}\left(\mathbf{0},\left(1,\left\{t t_{1}^{j} A_{j}^{(1)}, t t_{1}^{j-\frac{1}{2}} M_{j-\frac{1}{2}}^{(1)}\right\}_{j \in \mathbb{Z}_{+}}\right)\right)_{1} *_{0} \nu_{1}\left(\mathbf{0},\left(t_{1}^{\frac{1}{2}}, \mathbf{0}\right)\right)\right)(v)\right\rangle \\
& =\sum_{k \in \frac{1}{2} \mathbb{Z}} \sum_{i(k)=1}^{\operatorname{dim} V_{(k)}}\left\langle v^{\prime}, \nu_{1}\left(\mathbf{0},\left(1,\left\{t t_{1}^{j} A_{j}^{(1)}, t t_{1}^{j-\frac{1}{2}} M_{j-\frac{1}{2}}^{(1)}\right\}_{j \in \mathbb{Z}_{+}}\right)\right) e_{i^{(k)}}^{(k)}\right\rangle \\
& =\sum_{k \in \frac{1}{2} \mathbb{Z}} \sum_{i^{(k)}=1}^{\operatorname{dim} V_{(k)}}\left\langle v^{\prime}, \nu_{1}^{(k)}\left(\mathbf{0},\left(1,\left\{t t_{1}^{j} A_{j}^{(1)}, t t_{1}^{j-\frac{1}{2}} M_{j-\frac{1}{2}}\left(\mathbf{0},\left(t_{1}^{\frac{1}{2}}, \mathbf{0}\right)\right)(v)\right\rangle\right.\right.\right. \\
& \left.=\left\langle v^{\prime}, \nu_{1}\left(\mathbf{0},\left(1,\left\{t t_{1}^{j} A_{j}^{(1)}, t t_{1}^{j-\frac{1}{2}} M_{j-\frac{1}{2}}^{(1)}\right\}_{j \in \mathbb{Z}_{+}}\right)\right) t_{1}^{-L(k)}\right\rangle\right\rangle .
\end{aligned}
$$

When $v^{\prime} \in V_{(m)}^{\prime}$ and $v \in V_{(n)}$, combining (8.6) and (8.7), we have

$$
\left\langle v^{\prime}, R(t) v\right\rangle t_{1}^{-m}=\left\langle v^{\prime}, \nu_{1}\left(\mathbf{0},\left(1,\left\{t t_{1}^{j} A_{j}^{(1)}, t t_{1}^{j-\frac{1}{2}} M_{j-\frac{1}{2}}^{(1)}\right\}_{j \in \mathbb{Z}_{+}}\right)\right) v\right\rangle t_{1}^{-n} .
$$

By the supermeromorphicity axiom, $\left\langle v^{\prime}, \nu_{1}\left(\mathbf{0},\left(1,\left\{t t_{1}^{j} A_{j}^{(1)}, t t_{1}^{j-1 / 2} M_{j-1 / 2}^{(1)}\right\}_{j \in \mathbb{Z}_{+}}\right)\right) v\right\rangle$ is a power series in $t_{1}^{1 / 2}$. Then from (8.8), we conclude that $\left\langle v^{\prime}, R(t) v\right\rangle=0$ when 
$m>n$. From the positive energy axiom, it follows that $R(t)$ is an operator from $V$ to itself.

From Proposition 2.7 the sewing axiom and (8.5), we have

$$
\begin{aligned}
\left\langle v^{\prime},\right. & \left.R\left(t_{1}+t_{2}\right) v\right\rangle \\
& =\left\langle v^{\prime}, \nu_{1}\left(\mathbf{0},\left(1,\left(t_{1}+t_{2}\right)\left(A^{(1)}, M^{(1)}\right)\right)\right)(v)\right\rangle \\
& =\left\langle v^{\prime}, \nu_{1}\left(\left(\mathbf{0},\left(1, t_{1}\left(A^{(1)}, M^{(1)}\right)\right)\right)_{1} \infty_{0}\left(\mathbf{0},\left(1, t_{2}\left(A^{(1)}, M^{(1)}\right)\right)\right)\right)(v)\right\rangle \\
& =\left\langle v^{\prime},\left(\nu_{1}\left(\mathbf{0},\left(1, t_{1}\left(A^{(1)}, M^{(1)}\right)\right)\right)_{1} *_{0} \nu_{1}\left(\mathbf{0},\left(1, t_{2}\left(A^{(1)}, M^{(1)}\right)\right)\right)\right)(v)\right\rangle \\
& =\left\langle v^{\prime}, R\left(t_{1}\right) R\left(t_{2}\right) v\right\rangle
\end{aligned}
$$

for all $v \in V$ and $v^{\prime} \in V^{\prime}$. Thus

$$
R\left(t_{1}+t_{2}\right)=R\left(t_{1}\right) R\left(t_{2}\right)
$$

Since $\left\langle v^{\prime}, \nu_{1}\left(\mathbf{0},\left(1, t\left(A^{(1)}, M^{(1)}\right)\right)\right)(v)\right\rangle$ is a polynomial in $t$, so is $\left\langle v^{\prime}, R(t) v\right\rangle$. Hence $R(t) \in($ End $V)[[t]]$. This fact and equation (8.9) imply that there exists $X \in$ End $V$ such that $R(t)=e^{t X}$. Substituting this into equation [8.5), taking the derivative with respect to $t$ and then letting $t=0$, we obtain

$$
\left.\frac{\partial}{\partial t}\left\langle v^{\prime}, \nu_{1}\left(\mathbf{0},\left(1, t\left(A^{(1)}, M^{(1)}\right)\right)\right)(v)\right\rangle\right|_{t=0}=\left\langle v^{\prime}, X v\right\rangle .
$$

But from the definition of $G(j-1 / 2)$ and $L(j)$ for $j \in \mathbb{Z}_{+}$,

$$
\begin{aligned}
& \left.\frac{\partial}{\partial t}\left\langle v^{\prime}, \nu_{1}\left(\mathbf{0},\left(1, t\left(A^{(1)}, M^{(1)}\right)\right)\right)(v)\right\rangle\right|_{t=0} \\
& =\sum_{j \in \mathbb{Z}_{+}}\left(-A_{j}^{(1)}\left(-\left.\frac{\partial}{\partial\left(t A_{j}^{(1)}\right)}\left\langle v^{\prime}, \nu_{1}\left(\mathbf{0},\left(1, t\left(A^{(1)}, M^{(1)}\right)\right)\right)(v)\right\rangle\right|_{t\left(A^{(1)}, M^{(1)}\right)=\mathbf{0}}\right)\right. \\
& \left.\quad-M_{j-\frac{1}{2}}^{(1)}\left(-\left.\frac{\partial}{\partial\left(t M_{j-\frac{1}{2}}^{(1)}\right)}\left\langle v^{\prime}, \nu_{1}\left(\mathbf{0},\left(1, t\left(A^{(1)}, M^{(1)}\right)\right)\right)(v)\right\rangle\right|_{t\left(A^{(1)}, M^{(1)}\right)=\mathbf{0}}\right)\right) .
\end{aligned}
$$

Thus $X=-\sum_{j \in \mathbb{Z}_{+}}\left(-A_{j}^{(1)} L(j)+M_{j-1 / 2}^{(1)} G(j-1 / 2)\right)$ and hence

$$
\begin{aligned}
\left\langle v^{\prime}, \nu_{1}\left(\mathbf{0},\left(1, A^{(1)}, M^{(1)}\right)\right)(v)\right\rangle & =\left\langle v^{\prime}, e^{X} v\right\rangle \\
& \left.=\left\langle v^{\prime}, e^{-\sum_{j \in \mathbb{Z}_{+}}\left(A_{j}^{(1)} L(j)+M_{j-\frac{1}{2}}^{(1)} G\left(j-\frac{1}{2}\right)\right)} \cdot v\right)\right\rangle
\end{aligned}
$$

giving (8.3).

Similarly, by the supermeromorphicity axiom, $\left\langle v^{\prime}, \nu_{1}\left(\left(A^{(0)}, M^{(0)}\right),(1, \mathbf{0})\right)(v)\right\rangle$ is a polynomial in the $A_{j}^{(0)}$ 's and $M_{j-1 / 2}^{(0)}$ 's for $j \in \mathbb{Z}_{+}$. Thus $\left\langle v^{\prime}, \nu_{1}\left(\left(A^{(0)}, M^{(0)}\right),(1, \mathbf{0})\right)(v)\right\rangle$ is well defined for any $\left(A^{(0)}, M^{(0)}\right) \in \bigwedge_{\infty}^{\infty}$. In general $\left(\left(A^{(0)}, M^{(0)}\right),(1, \mathbf{0})\right) \in \tilde{S K}(1)$, i.e., it is a generalized supersphere with tubes. Since $\left\langle v^{\prime}, \nu_{1}\left(\left(A^{(0)}, M^{(0)}\right),(1, \mathbf{0})\right)(v)\right\rangle$ is linear in $v$ and $v^{\prime}$, there exists a linear operator $R^{\prime}(t)$ from $V^{\prime}$ to $\bar{V}^{*}$ such that

$$
\left\langle v^{\prime}, \nu_{1}\left(t\left(A^{(0)}, M^{(0)}\right),(1, \mathbf{0})\right)(v)\right\rangle=\left\langle R^{\prime}(t) v^{\prime}, v\right\rangle .
$$


We first show that, in fact, $R^{\prime}(t)$ is an operator from $V^{\prime}$ to itself. Using the sewing axiom and (8.4), we have

$$
\begin{aligned}
\left\langle v^{\prime},\right. & \left.\nu_{1}\left(\left(t\left(A^{(0)}, M^{(0)}\right),(1, \mathbf{0})\right)_{1} \infty_{0}\left(\mathbf{0},\left(t_{1}^{\frac{1}{2}}, \mathbf{0}\right)\right)\right)(v)\right\rangle \\
& =\left\langle v^{\prime},\left(\nu_{1}\left(t\left(A^{(0)}, M^{(0)}\right),(1, \mathbf{0})\right)_{1} *_{0} \nu_{1}\left(\mathbf{0},\left(t_{1}^{\frac{1}{2}}, \mathbf{0}\right)\right)\right)(v)\right\rangle \\
& =\sum_{k \in \frac{1}{2} \mathbb{Z}} \sum_{i^{(k)}=1}^{\operatorname{dim} V_{(k)}}\left\langle v^{\prime}, \nu_{1}\left(t\left(A^{(0)}, M^{(0)}\right),(1, \mathbf{0})\right) e_{i^{(k)}}^{(k)}\right\rangle\left\langle\left(e_{i(k)}^{(k)}\right)^{*}, \nu_{1}\left(\mathbf{0},\left(t_{1}^{\frac{1}{2}}, \mathbf{0}\right)\right)(v)\right\rangle \\
& =\sum_{k \in \frac{1}{2} \mathbb{Z}} \sum_{i^{(k)}=1}^{\operatorname{dim} V_{(k)}}\left\langle R^{\prime}(t) v^{\prime}, e_{i^{(k)}}^{(k)}\right\rangle\left\langle\left(e_{i^{(k)}}^{(k)}\right)^{*}, t_{1}^{-L(0)} v\right\rangle \\
& =\left\langle R^{\prime}(t) v^{\prime}, t_{1}^{-L(0)} v\right\rangle \\
(8.11) & =\left\langle R^{\prime}(t) v^{\prime}, t_{1}^{-L(0)} v\right\rangle .
\end{aligned}
$$

On the other hand, from

$$
\begin{aligned}
\left(t\left(A^{(0)}, M^{(0)}\right),(1, \mathbf{0})\right){ }_{1} \infty_{0}\left(\mathbf{0},\left(t_{1}^{\frac{1}{2}}, \mathbf{0}\right)\right) \\
=\left(\mathbf{0},\left(t_{1}^{\frac{1}{2}}, \mathbf{0}\right)\right){ }_{1} \infty_{0}\left(\left(\left\{t t_{1}^{j} A_{j}^{(0)}, t t_{1}^{j-\frac{1}{2}} M_{j-\frac{1}{2}}^{(0)}\right\}_{j \in \mathbb{Z}_{+}}\right),(1, \mathbf{0})\right),
\end{aligned}
$$

we have

$$
\begin{aligned}
\left\langle v^{\prime}, \nu_{1}\left(\left(t\left(A^{(0)}, M^{(0)}\right),(1, \mathbf{0})\right)_{1} \infty_{0}\left(\mathbf{0},\left(t_{1}^{\frac{1}{2}}, \mathbf{0}\right)\right)\right)(v)\right\rangle & \\
= & \left\langle v^{\prime}, \nu_{1}\left(\left(\mathbf{0},\left(t_{1}^{\frac{1}{2}}, \mathbf{0}\right)\right)_{1} \infty_{0}\left(\left(\left\{t t_{1}^{j} A_{j}^{(0)}, t t_{1}^{j-\frac{1}{2}} M_{j-\frac{1}{2}}^{(0)}\right\}_{j \in \mathbb{Z}_{+}}\right),(1, \mathbf{0})\right)\right)(v)\right\rangle \\
= & \left\langle v^{\prime},\left(\nu_{1}\left(\mathbf{0},\left(t_{1}^{\frac{1}{2}}, \mathbf{0}\right)\right)_{1} *_{0} \nu_{1}\left(\left(\left\{t t_{1}^{j} A_{j}^{(0)}, t t_{1}^{j-\frac{1}{2}} M_{j-\frac{1}{2}}^{(0)}\right\}_{j \in \mathbb{Z}_{+}}\right),(1, \mathbf{0})\right)\right)(v)\right\rangle \\
= & \sum_{k \in \frac{1}{2} \mathbb{Z}} \sum_{i^{(k)}=1}^{\operatorname{dim} V_{(k)}}\left\langle v^{\prime}, \nu_{1}\left(\mathbf{0},\left(t_{1}^{\frac{1}{2}}, \mathbf{0}\right)\right) e_{i^{(k)}}^{(k)}\right\rangle \\
= & \sum_{k \in \frac{1}{2} \mathbb{Z}} \sum_{i^{(k)}=1}^{\operatorname{dim} V_{(k)}}\left\langle\left(v_{i(k)}^{(k)}, t_{1}^{-L(0)} e_{i(k)}^{(k)}\right\rangle\right. \\
(8.12)= & \left\langle t_{1}^{-L^{\prime}(0)} v^{\prime}, \nu_{1}\left(\left(\left\{t t_{1}^{j} A_{j}^{(0)}, t t_{1}^{j-\frac{1}{2}} M_{j-\frac{1}{2}}^{(0)}\right\}_{j \in \mathbb{Z}_{+}}\right),(1, \mathbf{0})\right)(v)\right\rangle \\
& \left\langle\left(e_{i}^{j} A_{j}^{(k)}\right)^{*}, \nu_{1}\left(\left(\left\{t t_{1}^{j} A_{j}^{(0)}, t t t_{1}^{j-\frac{1}{2}} M_{j-\frac{1}{2}}^{(0)}\right\}_{j \in \mathbb{Z}_{+}}\right),(1, \mathbf{0})\right)(v)\right\rangle
\end{aligned}
$$

When $v^{\prime} \in V_{(m)}^{\prime}$ and $v \in V_{(n)}$, combining (8.11) and (8.12), we have

$$
\left\langle R^{\prime}(t) v^{\prime}, v\right\rangle t_{1}^{-n}=\left\langle v^{\prime}, \nu_{1}\left(\left(\left\{t t_{1}^{j} A_{j}^{(0)}, t t_{1}^{j-\frac{1}{2}} M_{j-\frac{1}{2}}^{(0)}\right\}_{j \in \mathbb{Z}_{+}}\right),(1, \mathbf{0})\right)(v)\right\rangle t_{1}^{-m} .
$$

By the supermeromorphicity axiom, $\left\langle v^{\prime}, \nu_{1}\left(\left(\left\{t t_{1}^{j} A_{j}^{(0)}, t t_{1}^{j-1 / 2} M_{j-1 / 2}^{(0)}\right\}_{j \in \mathbb{Z}_{+}}\right),(1, \mathbf{0})\right)(v)\right\rangle$ is a power series in $t_{1}^{1 / 2}$. Then from (8.13), we conclude that $\left\langle R^{\prime}(t) v^{\prime}, v\right\rangle=0$ when $m<n$. From the positive energy axiom, it follows that $R^{\prime}(t)$ is an operator from $V^{\prime}$ to itself. Thus the adjoint $R$ from $V$ to $V$ is well defined. From Proposition 2.7 
the sewing axiom and (8.10), we have

$$
\begin{aligned}
\left\langle R^{\prime}\right. & \left.\left(t_{1}+t_{2}\right) v^{\prime}, v\right\rangle \\
& =\left\langle v^{\prime}, \nu_{1}\left(\left(t_{1}+t_{2}\right)\left(A^{(0)}, M^{(0)}\right),(1, \mathbf{0})\right)(v)\right\rangle \\
& =\left\langle v^{\prime}, \nu_{1}\left(\left(\left(t_{1}\left(A^{(0)}, M^{(0)}\right),(1, \mathbf{0})\right)_{1} \infty_{0}\left(\left(t_{2}\left(A^{(0)}, M^{(0)}\right),(1, \mathbf{0})\right)\right)(v)\right\rangle\right.\right. \\
& =\left\langle v^{\prime},\left(\nu_{1}\left(\left(t_{1}\left(A^{(0)}, M^{(0)}\right),(1, \mathbf{0})\right)_{1} *_{0} \nu_{1}\left(\left(t_{2}\left(A^{(0)}, M^{(0)}\right),(1, \mathbf{0})\right)\right)(v)\right\rangle\right.\right. \\
& =\sum_{k \in \frac{1}{2} \mathbb{Z}} \sum_{i^{(k)}=1}^{\operatorname{dim} V_{(k)}}\left\langle R^{\prime}\left(t_{1}\right) v^{\prime}, e_{i^{(k)}}^{(k)}\right\rangle\left\langle R^{\prime}\left(t_{2}\right)\left(e_{i^{(k)}}^{(k)}\right)^{*}, v\right\rangle \\
= & \sum_{k \in \frac{1}{2} \mathbb{Z}} \sum_{i^{(k)}=1}^{\operatorname{dim} V_{(k)}}\left\langle R^{\prime}\left(t_{1}\right) v^{\prime}, e_{i^{(k)}}^{(k)}\right\rangle\left\langle\left(e_{i^{(k)}}^{(k)}\right)^{*}, R\left(t_{2}\right) v\right\rangle \\
= & \left\langle R^{\prime}\left(t_{1}\right) v^{\prime}, R\left(t_{2}\right) v\right\rangle \\
= & \left\langle R^{\prime}\left(t_{2}\right) R^{\prime}\left(t_{1}\right) v^{\prime}, v\right\rangle
\end{aligned}
$$

for all $v \in V$ and $v^{\prime} \in V^{\prime}$. Thus

$$
R^{\prime}\left(t_{1}+t_{2}\right)=R^{\prime}\left(t_{1}\right) R^{\prime}\left(t_{2}\right) .
$$

Since $\left\langle v^{\prime}, \nu_{1}\left(t\left(A^{(0)}, M^{(0)}\right),(1, \mathbf{0})\right)(v)\right\rangle$ is a polynomial in $t$, so is $\left\langle R^{\prime}(t) v^{\prime}, v\right\rangle$. Hence $R^{\prime}(t) \in\left(\right.$ End $\left.V^{\prime}\right)[[t]]$. This fact and equation 8.14) imply that there exists $X \in$ End $V^{\prime}$ such that $R^{\prime}(t)=e^{t X}$. Substituting this into equation (8.10), taking the derivative with respect to $t$ and then letting $t=0$, we obtain

$$
\left.\frac{\partial}{\partial t}\left\langle v^{\prime}, \nu_{1}\left(t\left(A^{(0)}, M^{(0)}\right),(1, \mathbf{0})\right)(v)\right\rangle\right|_{t=0}=\left\langle X v^{\prime}, v\right\rangle .
$$

But from the definition of $G(-j+1 / 2)$ and $L(-j)$ for $j \in \mathbb{Z}_{+}$,

$$
\begin{aligned}
\left.\frac{\partial}{\partial t}\left\langle v^{\prime}, \nu_{1}\left(t\left(A^{(0)}, M^{(0)}\right),(1, \mathbf{0})\right)(v)\right\rangle\right|_{t=0} \\
=\sum_{j \in \mathbb{Z}_{+}}\left(-A_{j}^{(0)}\left(-\left.\frac{\partial}{\partial\left(t A_{j}^{(0)}\right)}\left\langle v^{\prime}, \nu_{1}\left(t\left(A^{(0)}, M^{(0)}\right),(1, \mathbf{0})\right)(v)\right\rangle\right|_{t\left(A^{(0)}, M^{(0)}\right)=\mathbf{0}}\right)\right. \\
\left.\quad-M_{j-\frac{1}{2}}^{(0)}\left(-\left.\frac{\partial}{\partial\left(t M_{j-\frac{1}{2}}^{(0)}\right)}\left\langle v^{\prime}, \nu_{1}\left(t\left(A^{(0)}, M^{(0)}\right),(1, \mathbf{0})\right)(v)\right\rangle\right|_{t\left(A^{(0)}, M^{(0)}\right)=\mathbf{0}}\right)\right) \\
=-\sum_{j \in \mathbb{Z}_{+}}\left\langle v^{\prime}, A_{j}^{(0)} L(-j) v\right\rangle-\sum_{j \in \mathbb{Z}_{+}}\left\langle v^{\prime}, M_{j-\frac{1}{2}}^{(0)} G\left(-j+\frac{1}{2}\right) v\right\rangle .
\end{aligned}
$$

Thus $X=-\sum_{j \in \mathbb{Z}_{+}}\left(A_{j}^{(0)} L^{\prime}(j)+M_{j-1 / 2}^{(0)} G^{\prime}(j-1 / 2)\right)$, and therefore

$$
\begin{aligned}
\left\langle v^{\prime}, \nu_{1}\left(\left(A^{(0)}, M^{(0)}\right),(1,0)\right)(v)\right\rangle & =\left\langle e^{X} v^{\prime}, v\right\rangle \\
& =\left\langle e^{-\sum_{j \in \mathbb{Z}_{+}}\left(A_{j}^{(0)} L^{\prime}(j)+M_{j-\frac{1}{2}}^{(0)} G^{\prime}\left(j-\frac{1}{2}\right)\right)} \cdot v^{\prime}, v\right\rangle
\end{aligned}
$$

giving (8.2). Hence $F_{S V} \circ F_{S G}=1_{S G}$. 
Finally, that $F_{S G} \circ F_{S V}=1_{S V_{\varphi}}$ on objects is equivalent to $(V, Y(\cdot,(x, \varphi)), \mathbf{1}, \tau)=$ $\left(V, Y_{\nu^{Y}}(\cdot,(x, \varphi)), \mathbf{1}_{\nu^{Y}}, \tau_{\nu^{Y}}\right)$. But this is obvious since by definition

$$
\begin{aligned}
\left.\left\langle v^{\prime}, Y_{\nu^{Y}}\left(v_{1},(x, \varphi)\right) v_{2}\right\rangle\right|_{(x, \varphi)=(z, \theta)} & =\left\langle v^{\prime}, \nu_{2}^{Y}((z, \theta) ; \mathbf{0},(1, \mathbf{0}),(1, \mathbf{0}))\left(v_{1} \otimes v_{2}\right)\right\rangle \\
& =\left.\left\langle v^{\prime}, Y\left(v_{1},(x, \varphi)\right) v_{2}\right\rangle\right|_{(x, \varphi)=(z, \theta)}, \\
\left\langle v^{\prime}, \mathbf{1}_{\nu^{Y}}\right\rangle & =\left\langle v^{\prime}, \nu_{0}^{Y}(\mathbf{0})\right\rangle \\
& =\left\langle v^{\prime}, \mathbf{1}\right\rangle,
\end{aligned}
$$

and

$$
\begin{aligned}
\left\langle v^{\prime}, \tau_{\nu^{Y}}\right\rangle & =\left\langle v^{\prime},-\frac{\partial}{\partial \epsilon} \nu_{0}^{Y}\left(\mathbf{0}, M\left(\epsilon, \frac{3}{2}\right)\right)\right\rangle \\
& =\left\langle e^{\epsilon G^{\prime}\left(\frac{3}{2}\right)} v^{\prime}, \mathbf{1}\right\rangle \\
& =\left\langle G^{\prime}\left(\frac{3}{2}\right) v^{\prime}, \mathbf{1}\right\rangle \\
& =\left\langle v^{\prime}, G\left(-\frac{3}{2}\right) \mathbf{1}\right\rangle \\
& =\left\langle v^{\prime}, \tau\right\rangle .
\end{aligned}
$$

Therefore $F_{S G} \circ F_{S V}=1_{S V_{\varphi}}$.

Remark 8.2. The results of this paper subsume the correspondence between geometric vertex operator algebras and vertex operator algebras developed in [H2], assuming the convergence of the central series $\Gamma$, i.e., assuming Conjecture 3.5

\section{REFERENCES}

[B1] K. Barron, A supergeometric interpretation of vertex operator superalgebras, Int. Math. Res. Notices, 1996 No. 9, Duke University Press, 409-430.

[B2] K. Barron, The supergeometric interpretation of vertex operator superalgebras, Ph.D. thesis, Rutgers University, 1996.

[B3] K. Barron, " $N=1$ Neveu-Schwarz vertex operator superalgebras over Grassmann algebras and with odd formal variables" in Representations and Quantizations: Proceedings of the International Conference on Representation Theory, July 1998, Shanghai China, SpringerVerlag, 9-39.

[B4] K. Barron, The moduli space of $N=1$ superspheres with tubes and the sewing operation, Memoirs Amer. Math. Soc., to appear in 2003.

[B5] K. Barron, Change of variables for $N=1$ Neveu-Schwarz vertex operator superalgebras, in preparation.

[B6] K. Barron, Alternate notions of $N=1$ superconformality and relationships to $N=2$ NeveuSchwarz vertex operator superalgebras, in preparation.

[BHL] K. Barron, Y.-Z. Huang and J. Lepowsky, Factorization of formal exponentials and uniformization, J. Alg. 228 (2000), 551-579.

[BPZ] A. A. Belavin, A. M. Polyakov and A. B. Zamolodchikov, Infinite conformal symmetries in two-dimensional quantum field theory, Nuclear Phys. B 241 (1984), 333-380.

[Bo] R. Borcherds, Vertex algebras, Kac-Moody algebras, and the Monster, Proc. Nat. Acad. Sci. USA 83 (1986), 3068-3071.

[CR] L. Crane and J. Rabin, Super Riemann surfaces: uniformization and Teichmüller theory, Commun. Math. Phys. 113 (1988), 601-623.

[D] B. DeWitt, Supermanifolds, 2nd ed., Cambridge Monogr. Math. Phys., Cambridge Univ. Press, Cambridge, 1992.

[DL] C. Dong and J. Lepowsky, Generalized Vertex Algebras and Relative Vertex Operators, Progr. Math., 112, Birkhäuser, Boston, 1993.

[FFR] A. J. Feingold, I. B. Frenkel and J. F. X. Ries, Spinor Construction of Vertex Operator Algebras, Triality and $E_{8}^{(1)}$, Contemp. Math. 121, Amer. Math. Soc., Providence, 1991. 
[FG] W. Fischer and H. Grauert, Lokal-triviale Familien kompakter komplexer Mannigfaltigkeiten, Nachr. Akad. Wiss. Göttingen Math.-Phys. Kl. 1965, 89-94.

[Fk] I. B. Frenkel, talk presented at the Institute for Advanced Study, 1988.

[FHL] I. B. Frenkel, Y.-Z. Huang and J. Lepowsky, On axiomatic approaches to vertex operator algebras and modules, Memoirs Amer. Math. Soc. 104, Num. 494, 1993.

[FLM] I. B. Frenkel, J. Lepowsky and A. Meurman, Vertex Operator Algebras and the Monster, Academic Press, New York, 1988.

[Fd] D. Friedan, "Notes on string theory and two dimensional conformal field theory" in Unified String Theories, World Scientific, Singapore, 1986, 162-213.

[FS] D. Friedan and S. Shenker, The analytic geometry of two-dimensional conformal field theory, Nuclear Phys. B 281 (1987), 509-545.

[G] P. Goddard, "Meromorphic conformal field theory" in Infinite-Dimensional Lie Algebras and Groups, ed. by V. Kac, Adv. Ser. Math. Phys. 7, World Scientific, Teaneck, N.J., 1989, $556-587$.

[GKO] P. Goddard, A. Kent and D. Olive, Unitary Representations of the Virasoro and SuperVirasoro Algebras, Commun. Math. Phys. 103 (1986), 105-119.

[H1] Y.-Z. Huang, On the geometric interpretation of vertex operator algebras, Ph.D. thesis, Rutgers University, 1990.

[H2] Y.-Z. Huang, Two Dimensional Conformal Geometry and Vertex Operator Algebras, Birkhäuser, Boston, 1997.

[HL1] Y.-Z. Huang and J. Lepowsky, "Vertex operator algebras and operads", in: The Gelfand Mathematical Seminar, 1990-1992, ed. L. Corwin, I. Gelfand and J. Lepowsky, Birkhäuser, Boston, 1993, 145-161.

[HL2] Y.-Z. Huang and J. Lepowsky, "Operadic formulation of the notion of vertex operator algebra", in: Mathematical Aspects of Conformal and Topological Field Theories and Quantum Groups, ed. P. Sally, M. Flato, J. Lepowsky, N. Reshetikhin and G. Zuckerman, Contemp. Math. 175, Amer. Math. Soc., Providence, 1994, 131-148.

[K] V. Kac, Lie superalgebras, Adv. Math. 26 (1977), 8-96.

$[\mathrm{KW}]$ V. Kac and W. Wang, "Vertex operator superalgebras and their representations", in Mathematical Aspects of Conformal and Topological Field Theories and Quantum Groups, ed. by P. Sally, M. Flato, J. Lepowsky, N. Reshetikhin and G. Zuckerman, Contemp. Math. 175, Amer. Math. Soc., Providence, 1994, 161-191.

[M] J. P. May, The geometry of iterated loop spaces, Lecture Notes in Math., No. 271, SpringerVerlag, Berlin, 1972.

[NS] A. Neveu and J. H. Schwarz, Factorizable dual model of pions, Nucl. Phys. B 31 (1971), 86-112.

[R] A. Rogers, Graded manifolds, supermanifolds and infinite-dimensional Grassmann algebras, Commun. Math. Phys. 105 (1986), 375-384

[S] G. Segal, The definition of conformal field theory, preprint.

[T] H. Tsukada, Vertex operator superalgebras, Comm. Algebra 18 (1990), 2249-2274.

[V] C. Vafa, Conformal theories and punctured surfaces, Phys. Lett. B 199 (1987), 195-202.

Department of Mathematics, 255 Hurley Hall, University of Notre Dame, Notre DAME IN 46556

E-mail address: kbarron@nd.edu 